Enhancing aroma production by lactic acid bacteria at near-zero growth rates:

4 a retentostaţ approach

Oscar van Mastrigt
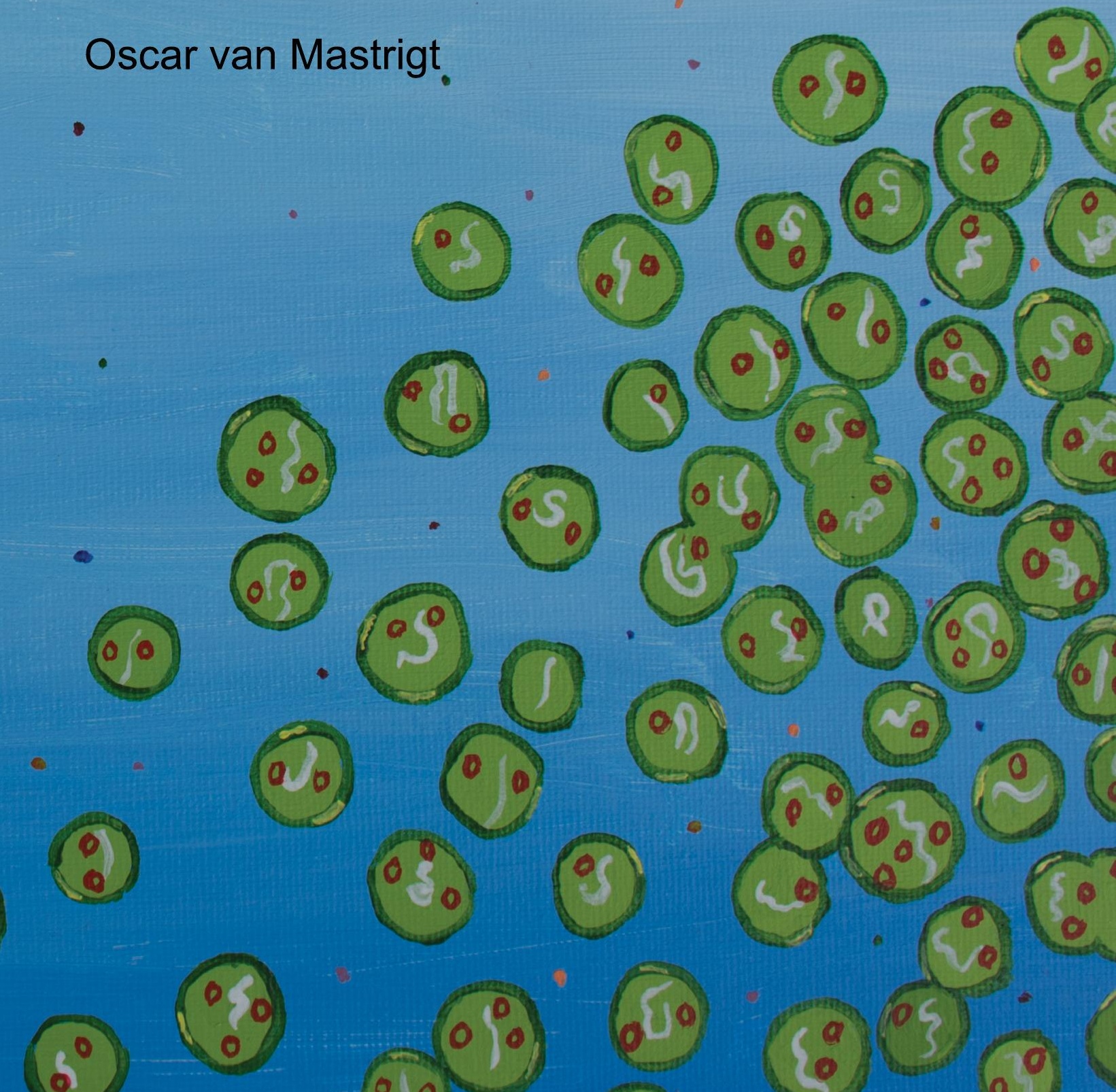


\section{Propositions}

1. Production of typical cheese aroma compounds requires slowly growing bacteria.

(this thesis)

2. The maintenance requirement of dairy lactic acid bacteria is growth-rate dependent.

(this thesis)

3. Malware encoded by DNA sequences (Ney, P. et al. 2017. 26th USENIX Security Symposium, https://dnasec.cs.washington. edu/dnasec.pdf) poses a privacy risk in DNA sequencing.

4. Connections via nanotubes (Stempler et al. 2017. Interspecies nutrient extraction and toxin delivery between bacteria. Nat Comm 8:315) shed new light on interactions between microbial cells.

5. Lack of consumer acceptance drives food innovation.

6. The number of students at universities is regulated by negative feedback.

Propositions belonging to the thesis, entitled

Enhancing aroma production by lactic acid bacteria at near-zero growth rates: a retentostat approach

Oscar van Mastrigt

Wageningen, 29 August 2018 
Enhancing aroma production by lactic acid bacteria at near-zero growth rates:

a retentostat approach

Oscar van Mastrigt 


\section{Thesis committee}

\section{Promotors}

Prof. Dr E. J. Smid

Personal chair at the Laboratory of Food Microbiology

Wageningen University \& Research

Prof. Dr T. Abee

Personal chair at the Laboratory of Food Microbiology

Wageningen University \& Research

\section{Other members}

Prof. Dr R. van Kranenburg, Wageningen University \& Research

Prof. Dr P. Daran-Lapujade, Delft University of Technology

Prof. Dr J. Hugenholtz, University of Amsterdam

Dr M. Serrano, CSK Food Enrichment, Wageningen

This research was conducted under the auspice of Graduate School VLAG (Advanced studies in Food Technology, Agrobiotechnology, Nutrition and Health Sciences) 


\title{
Enhancing aroma production by lactic acid bacteria at near-zero growth rates: a retentostat approach
}

\author{
Oscar van Mastrigt
}

\section{Thesis}

submitted in fulfilment of the requirements for the degree of doctor at Wageningen University by the authority of the Rector Magnificus, Prof. Dr A. P. J. Mol, in the presence of the Thesis Committee appointed by the Academic Board to be defended in public on Wednesday 29 August 2018 at $1: 30$ p.m. in the Aula. 
Oscar van Mastrigt

Enhancing aroma production by lactic acid bacteria at near-zero growth rates: $a$ retentostat approach,

236 pages.

PhD thesis, Wageningen University, Wageningen, the Netherlands (2018)

With references, with summary in English

ISBN 978-94-6343-300-6

DOI https://doi.org/10.18174/453531 


\section{Table of contents}

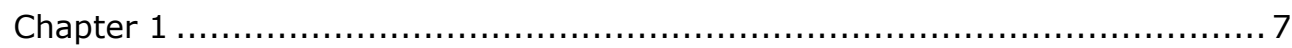

General introduction

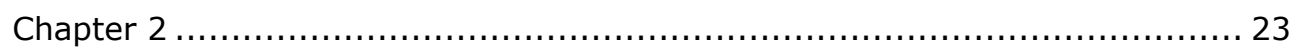

Complete genome sequences of Lactococcus lactis subsp. lactis bv diacetylactis FM03 and Leuconostoc mesenteroides FM06 isolated from cheese

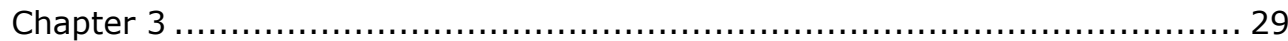

Large plasmidome of dairy Lactococcus lactis subsp. lactis biovar diacetylactis FM03P encodes technological functions and appears highly unstable

Chapter 4

Citrate, low pH and amino acid limitation induce citrate utilisation in Lactococcus lactis biovar diacetylactis

Chapter 5

Aroma formation during cheese ripening is best resembled by Lactococcus lactis retentostat cultures

Chapter 6

Quantitative physiology and aroma formation of a dairy Lactococcus lactis at near-zero growth rates

Chapter 7 145

Aroma formation in retentostat co-cultures of Lactococcus lactis and Leuconostoc mesenteroides

Chapter 8 167

Dynamics in copy numbers of five plasmids of a dairy Lactococcus lactis strain under dairy-related conditions including near-zero growth rates

Chapter 9

Application of a partial cell recycling chemostat for continuous production of aroma compounds at near-zero growth rates

Chapter 10 201

General discussion

Summary 223

Acknowledgements . 227

About the author 231

List of publications. 233

Overview of training activities 235 



\section{Chapter 1}

General introduction 


\section{General introduction}

Fermentation has already been used for thousands of years to preserve food (McGovern et al., 2004, Salque et al., 2012, Wang et al., 2016), but only since the second half of the $19^{\text {th }}$ century (Pasteur, 1857a, b) people realised that microorganisms play a crucial role in fermentation processes. Nowadays, starter bacteria and yeasts are still used as work horses for the production of many foods, such as cheese, yoghurt, bread, beer and wine. And microbes are not only used to preserve food, but their biotechnological application has expanded to production of many microbial products, such as food ingredients, fine chemicals, enzymes and pharmaceuticals.

\section{Cheese production}

The earliest evidence of cheese making has been dated back to the sixth millennium BC in Northern Europe (Salque et al., 2012). At that time fermentation of milk relied on spontaneous acidification by microbes naturally present in the raw milk, which consisted mainly of lactic acid bacteria (LAB). Relying on the microbes naturally present could lead to unpredictable results affecting the product quality (Parente et al., 2017). Therefore, to assure reproducibility part of the previous day's batch can be used to start a new fermentation. This practice is called backslopping and is still carried out in the production of some traditional cheeses, like Parmigiano-Reggiano (Fox and McSweeney, 2017). This backslopping procedure allows the bacteria to adapt to the environment resulting in faster acidification and utilisation of the main substrates in milk (lactose, citrate and caseins).

Nowadays, most cheeses are made with starter cultures. These starter cultures usually consist of a mixture of LAB strains originating from successful batches of fermented dairy products or from natural whey starters obtained by backslopping. The main role of the starter cultures is to acidify the milk by conversion of lactose into lactic acid, which contributes to the safety and shelf life of the product. Moreover, starter cultures contribute to the flavour development during the ripening process which involves proteolysis, lipolysis and the utilisation of lactose and citrate (Smid and Kleerebezem, 2014).

Starter cultures consisting of LAB are classified as thermophilic and mesophilic and are used for cheese varieties where a high temperature $\left(>37^{\circ} \mathrm{C}\right.$ but generally $48-52^{\circ} \mathrm{C}$ ) prevails during the early stages of cheese manufacturing, like Italian and Swiss cheese, or a moderate temperature (below $40^{\circ} \mathrm{C}$ ), like Dutch-type cheese (Parente et al., 2017). The mesophilic starter cultures consist of various 
strains of Lactococcus lactis subsp. cremoris, L. lactis subsp. lactis, L. lactis subsp. lactis biovar diacetylactis and Leuconostoc mesenteroides with different metabolic capacities and different functional roles (Smid et al., 2014). Proteolytic strains break down the caseins releasing peptides and amino acids that can be consumed by the entire microbial community. L. lactis subsp. cremoris strains are the most fastidious and are mainly involved in the fast acidification of the milk. Moreover, they quickly lyse during cheese ripening releasing enzymes accelerating peptidolytic reactions (Chapot-Chartier et al., 1994). L. lactis subsp. lactis is characterised by its ability to grow in the presence of $4 \% \mathrm{NaCl}$, utilise arginine as energy source and has glutamate decarboxylase activity producing $\mathrm{Y}^{-}$ aminobutyric acid (GABA) (Rademaker et al., 2007). L. lactis subsp. lactis survives longer during the ripening process and is involved in flavour formation. Both $L$. lactis subsp. lactis biovar diacetylactis and Lc. mesenteroides strains are able to ferment citrate and convert it into the important aroma compounds diacetyl and acetoin, which are responsible for the buttery note, and $\mathrm{CO}_{2}$, which is important for eye formation (Hugenholtz, 1993). In contrast to the homofermentative $L$. lactis, LC. mesenteroides is a heterofermentative organism thus also producing $\mathrm{CO}_{2}$ via lactose fermentation (Fig. 1.1). Both LC. mesenteroides and $L$. lactis biovar diacetylactis represent a small fraction of the starter cultures (1-10\%) (Erkus et al., 2013), but become dominant during cheese ripening and are considered to be the main aroma producers in the mesophilic starter cultures.

\section{Adaptation via plasmid acquisition}

The long history of cheese making using backslopping allowed the lactic acid bacteria to adapt to the dairy environment. Typical adaptations to the nutrientrich dairy environment are genome decay, particularly for L. lactis subsp. cremoris, and the acquisition of plasmids (Kelleher et al., 2017). For instance, dairy isolates often have auxotrophies for branched-chain amino acids (BCAA) and histidine (Delorme et al., 1993, Godon et al., 1993), which are the most abundant amino acids released by casein degradation. Plasmids in L. lactis and LC. mesenteroides have been linked to important dairy phenotypes including lactose metabolism, citrate uptake, protein degradation and peptide uptake, bacteriocin production, exopolysaccharide production and stress and bacteriophage resistance (Ainsworth et al., 2014) (Table 1.1). The importance of plasmids for growth and survival of $L$. lactis in the dairy environment is also demonstrated in Chapter 3 showing an inventory of plasmid-encoded functionalities. Especially plasmids greatly increase the genetic diversity as they can make up $9 \%$ of the genetic repertoire of the bacteria (Ainsworth et al., 2014). 


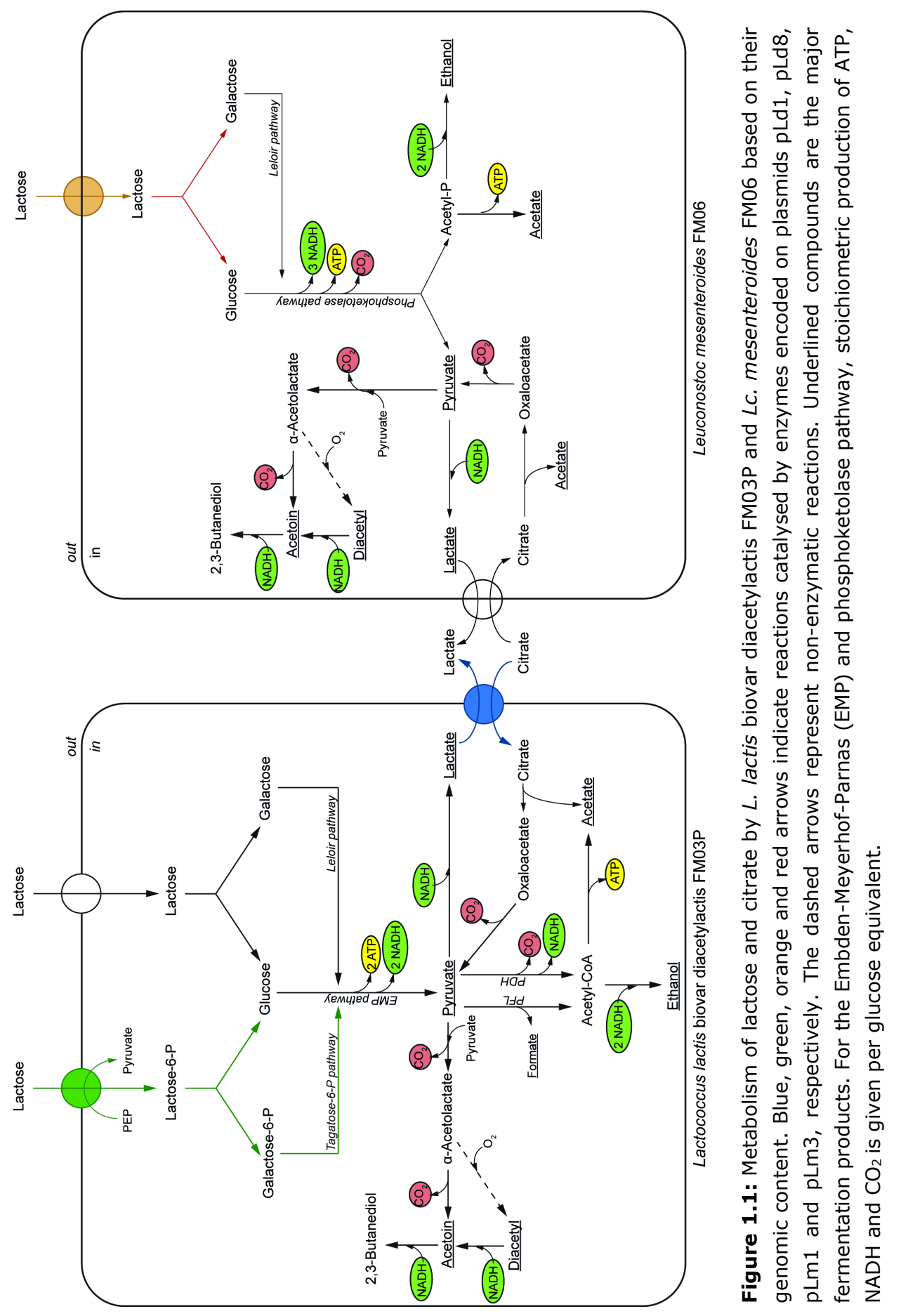


Table 1.1: Plasmid-encoded functions in $L$. lactis and $L C$. mesenteroides.

\begin{tabular}{|c|c|c|}
\hline Function & Genes & References \\
\hline Lactose utilisation & $\begin{array}{l}\text { lacR-ABCDFEGX (L. lactis) } \\
\text { lacS, lacLM (Lc. mesenteroides) }\end{array}$ & $\begin{array}{l}\text { (David et al., 1992, de Vos et } \\
\text { al., 1990, van Rooijen and de } \\
\text { Vos, 1990, van Rooijen et al., } \\
\text { 1991, Vaughan et al., 1996) }\end{array}$ \\
\hline Citrate uptake & citQRP (L. lactis) & (de Felipe et al., 1995) \\
\hline Protease & prtP-prtM (L. lactis) & $\begin{array}{l}\text { (de Vos et al., 1989, Vos et al., } \\
1989 \text { ) }\end{array}$ \\
\hline Peptidase & $p e p F, p e p O, p c p$ & $\begin{array}{l}\text { (Nardi et al., 1997, Siezen et } \\
\text { al., 2005) }\end{array}$ \\
\hline Peptide transport & oppDFBCA & $\begin{array}{l}\text { (Tynkkynen et al., 1993, Yu et } \\
\text { al., 1996) }\end{array}$ \\
\hline Bacteriophage resistance & & \\
\hline Restriction-modification & $h s d M, h s d R, h s d S$ & (Schouler et al., 1998) \\
\hline Abortive infection & $a b i$ & $\begin{array}{l}\text { (Klaenhammer and Sanozky, } \\
\text { 1985) }\end{array}$ \\
\hline $\begin{array}{l}\text { Exopolysaccharide } \\
\text { production }\end{array}$ & eps & $\begin{array}{l}\text { (Forde and Fitzgerald, 1999, } \\
\text { van Kranenburg et al., 1997, } \\
\text { van Kranenburg et al., 2000) }\end{array}$ \\
\hline Bacteriocin production & & $\begin{array}{l}\text { (Choi and Ahn, 1997, Davey, } \\
\text { 1984, Gasson, 1984, Harmon } \\
\text { and McKay, 1987, Holo et al., } \\
\text { 1991, Scherwitz et al., 1983) }\end{array}$ \\
\hline \multicolumn{3}{|l|}{ Ion uptake } \\
\hline $\mathrm{Mg}^{2+}$ & corA & (Siezen et al., 2005) \\
\hline $\mathrm{Mn}^{2+}$ & $\mathrm{mntH}$ & (Siezen et al., 2005) \\
\hline \multicolumn{3}{|l|}{ Heavy metal resistance } \\
\hline $\mathrm{Cd}^{2+}$ & CadCA & (Liu et al., 1997) \\
\hline $\mathrm{Cu}^{2+}$ & ICORSABS & $\begin{array}{l}\text { (Leelawatcharamas et al., } \\
\text { 1997, Liu et al., 2002) }\end{array}$ \\
\hline Glutamate dehydrogenase & $g d h$ & $\begin{array}{l}\text { (Fallico et al., 2011, Tanous et } \\
\text { al., 2005) }\end{array}$ \\
\hline Stress resistance & usp $A, \operatorname{csp} C, \operatorname{csp} D, \operatorname{cst} A$ & (Siezen et al., 2005) \\
\hline
\end{tabular}

\section{Aroma formation during cheese ripening}

Ripening of cheese is a complex process in which the typical cheese characteristics, such as the flavour and texture, are formed by the action of numerous enzymes derived from the milk, the rennet, the starter bacteria and the non-starter bacteria (Fox et al., 2017). Cheese ripening is a slow process that can last from 2 weeks to several years for Mozzarella and Parmigiano-Reggiano, respectively (McSweeney, 2004). The temperature is kept low to prevent growth of spoilage and pathogenic microorganisms making this process not only timeconsuming but also expensive. Flavour formation during cheese ripening consists of two steps: i) the generation of precursors from carbohydrates, proteins and fats and $\mathrm{ii}$ ) their conversion into a wide range of compounds responsible for the flavour and texture of the cheese (Smid and Kleerebezem, 2014).

Caseins can be degraded into peptides by rennet enzymes and the extracellular, cell envelope-bound proteinase PrtP of Lactococcus lactis (de Vos et al., 1989). Subsequently, these peptides are taken up by several di-, tri- and oligopeptide 
transporters of L. lactis and Lc. mesenteroides (Juillard et al., 1995, Smid et al., 1991) and hydrolysed by intracellular peptidases releasing the free amino acids involved in flavour formation (Kunji et al., 1996). In particular, methionine, aromatic and branched-chain amino acids are crucial in flavour development as they can be converted into various alcohols, aldehydes, acids, esters and sulphur compounds (Smit et al., 2005). Amino acid catabolism (Fig. 1.2) starts with an aminotransferase reaction forming the amino acid-derived a-keto acid. Subsequently, the a-keto acid can be decarboxylated to its corresponding aldehyde and further oxidised or reduced to the corresponding acid or alcohol. Alternatively, a-keto acids can be converted to carboxylic acids via oxidative decarboxylation generating ATP (Yvon and Rijnen, 2001). This consist of 3 steps: conversion to acyl-CoAs by a dehydrogenase complex, followed by a transacetylase and a kinase reaction resulting in the carboxylic acid and ATP (Chambellon et al., 2009). Finally, acids and alcohols can participate in ester formation. Methionine can also be converted directly into methanethiol by cystathione $\beta$-lyase (Alting et al., 1995). Most of these reactions are catalysed by specific enzymes, although some chemical reactions have been reported such as benzaldehyde formation from phenylpyruvic acid (Nierop Groot and de Bont, 1998, Spus et al., 2017) or methanethiol formation from a-keto methylthio butyric acid (Bonnarme et al., 2004).

Milk fat is essential for the development of flavour during cheese ripening as demonstrated in studies in which cheese was made with skimmed milk (Collins et al., 2003). The lipids are hydrolysed by lipolytic enzymes that could originate from the milk, the rennet or the bacteria and lead to the formation of glycerol and free fatty acids (FFAs). FFAs contribute directly to the cheese flavour, in particular the short and medium-chain FFAs, but can also be converted via $\beta$-oxidation into flavour compounds such as methylketones, secondary alcohols, esters and lactones (Collins et al., 2003). In general, lipolytic activity of LAB is low (Holland and Coolbear, 1996) and lipolysis mainly plays a role in surface-ripened cheese in which lipases are delivered by moulds (Molimard and Spinnler, 1996) or by coryneform bacteria (e.g. Brevibacterium linens) (Sørhaug and Ordal, 1974) in bacterial surface-ripened cheese. However, in Cheddar and Dutch-type cheese lipases of LAB are probably the principal lipolytic agents (Fox et al., 2000). Because lipases of LAB appear to be exclusively located intracellular (Fernández et al., 2000), lipolysis might be affected by cell lysis during ripening of these cheeses. 


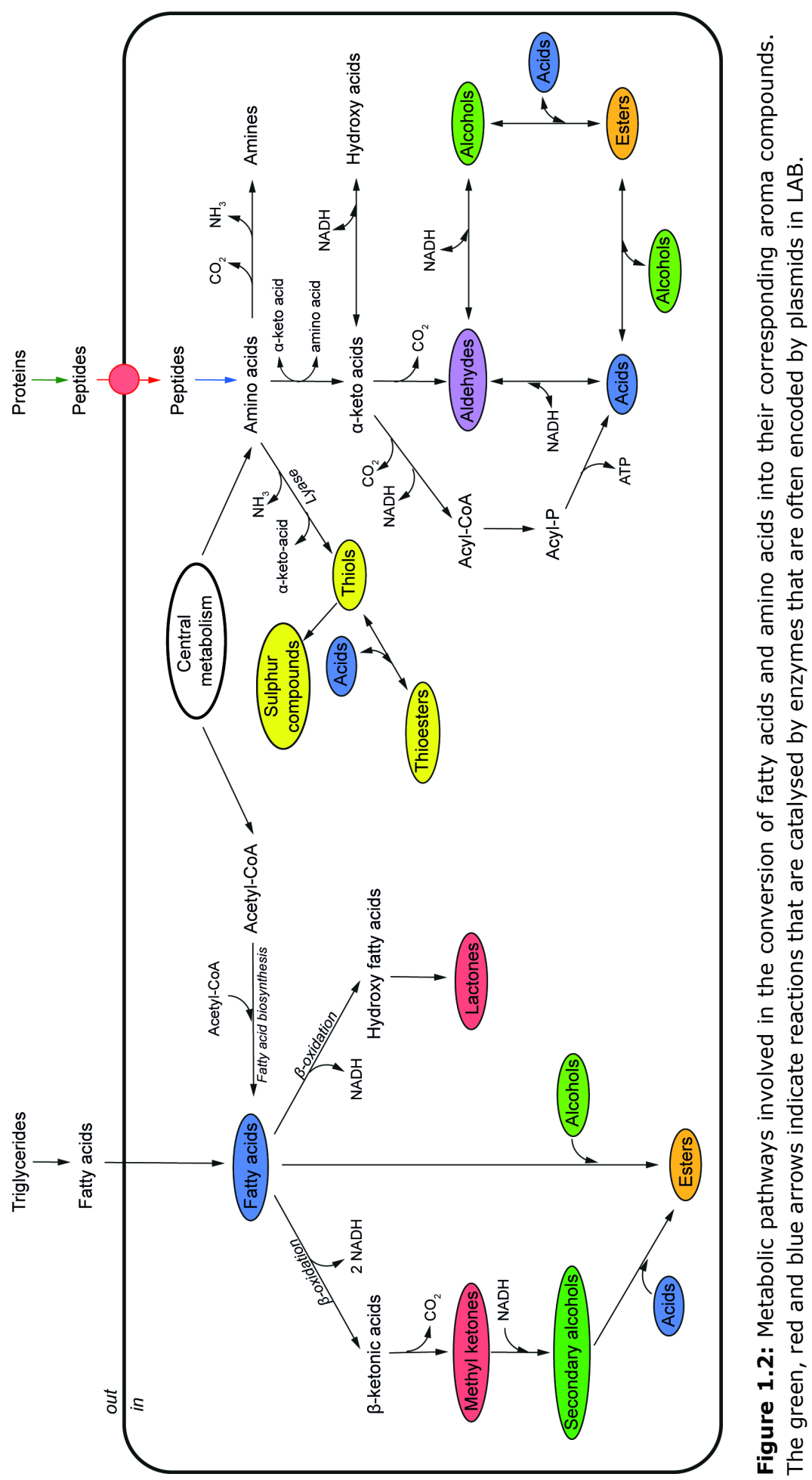


As described before, citrate is an important precursor for the production of the C4 aroma compounds diacetyl and acetoin. Only a few LAB species are able to utilise citrate, such as L. lactis biovar diacetylactis and LC. mesenteroides (Hugenholtz, 1993). Citrate utilisation has been linked to the presence of the plasmid-encoded citrate permease gene citP in L. lactis (Kempler and Mckay, 1979). This secondary transporter exchanges divalent citrate with monovalent L-lactate during citrateglucose co-metabolism (Bandell et al., 1998) (Fig. 1.1). Intracellularly, citrate is first converted into acetate and oxaloacetate by citrate lyase and subsequently the oxaloacetate is decarboxylated into pyruvate and $\mathrm{CO}_{2}$. This leads to an increased pool of intracellular pyruvate favouring the reaction towards aacetolactate by acetolactate synthase, which has a low affinity for pyruvate $\left(\mathrm{K}_{\mathrm{m}}=\right.$ $50 \mathrm{mM}$ ) (Snoep et al., 1992). Finally, the a-acetolactate can be converted to acetoin via acetolactate decarboxylase or chemically oxidised to diacetyl. Citrate metabolism and conversion into acetoin and diacetyl is induced at low pH due to increased expression of citrate permease, citrate lyase, oxaloacetate decarboxylase and acetolactate synthase (García-Quintáns et al., 1998, García-

Quintáns et al., 2008, Martín et al., 2004, Sender et al., 2004) indicating that citrate metabolism is an important resistance mechanism towards acid stress by increasing the intracellular $\mathrm{pH}$. This may also explain the relatively good survival of citrate utilizing lactic acid bacteria during cheese ripening (Erkus et al., 2013).

\section{Mimicking cheese ripening using retentostat cultivation}

During cheese ripening, nutrients and in particular fermentable carbohydrates are scarce leading to severe reduction in the growth rate of the lactic acid bacteria. However, particular bacteria remain viable and still contribute to aroma formation. To mimic the extreme nutrient limitation during cheese ripening, retentostat cultivation can be used (Fig. 1.3). This is a modification of the well-known chemostat cultivation in which a biomass filter is connected to the effluent line to retain the biomass in the bioreactor. Thereby, the biomass concentration will increase. Because the nutrient supply will be constant, more cells have to share the same amount of nutrients leading to lower growth rates. At some point, the nutrient supply will equal the energy required for the maintenance processes of the cells and no energy is left for growth. This situation we call zero growth. Zero growth is fundamentally different from starvation conditions because the cells can still maintain themselves and remain viable. 

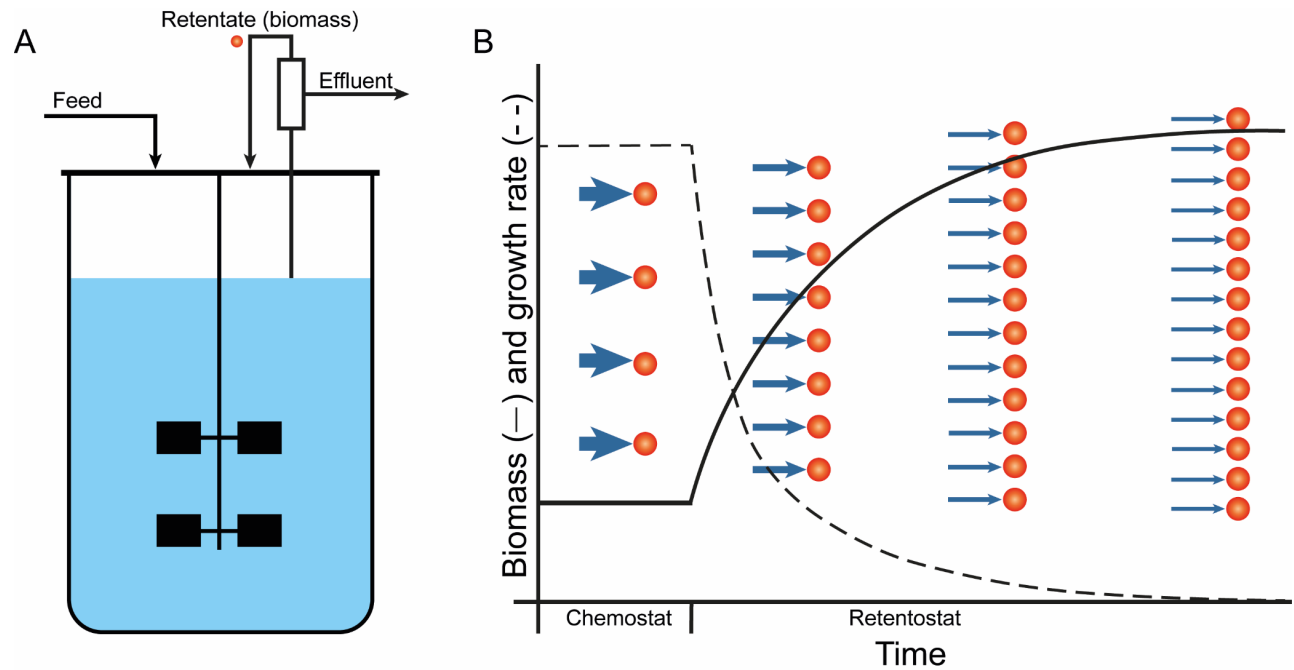

Figure 1.3: Retentostat cultivation. A: Schematic overview of the retentostat cultivation set-up. Medium is continuously supplied at a fixed rate (feed) and effluent is removed via a filter to retain the biomass inside the bioreactor. B: Principle of retentostat cultivation. After a steady state is achieved in the chemostat, the filter is connected to the effluent line resulting in an increase in biomass (black line and red cells) in time. Because the feed rate is constant, the substrate availability per cell (blue arrows) decreases, and the growth rate reduces (dashed line). After some time, the substrate availability will equal the maintenance requirements of the cells and no substrate is left for growth. This situation we call zero growth.

Retentostat cultivation has been successfully applied to study the physiological and transcriptional responses of several industrially relevant microorganisms towards near-zero growth rates, including Lactococcus lactis, Lactobacillus plantarum, Bacillus subtilis, Saccharomyces cerevisiae and Aspergillus niger (Ercan et al., 2015). Biomass accumulation profiles in the retentostat cultivations suggested that the growth rate of all microbes reduced to less than $0.001 \mathrm{~h}^{-1}$ corresponding to doubling times of more than a month, except for $A$. niger, which formed conidiospores that could pass the filter. Meanwhile, the viability remained above $80 \%$. Physiological and transcriptional responses were distinct from those induced by starvation in stationary-phase cultures. General responses included a stress response, increased stress resistance, alternative carbon-source utilisation and downregulation of protein synthesis (Ercan et al., 2015). Moreover, speciesspecific responses towards near-zero growth rates were observed including downregulation of the expression of genes involved in motility and chemotaxis in $B$. subtilis, reduced exopolysaccharide expression in L. lactis, production of plant growth-stimulation compounds in $L b$. plantarum, increase in surface-to-volume ratio in $B$. subtilis and $L$. lactis and upregulation of genes characteristic for 
quiescent cells. These studies highlight the power of retentostat cultivation to study microorganisms at near-zero growth rates. Because during cheese ripening nutrients are limited and $L A B$ hardly grow, retentostat cultivation might also be applied to study LAB under conditions mimicking the cheese ripening environment.

As described before, dairy lactic acid bacteria often carry plasmids important for growth and survival in the dairy environment. However, it remains unknown what happens to plasmids and their copy number at near-zero growth rates. Moreover, the described studies mainly looked only at production of the primary metabolites, while in dairy processes food characteristics are mainly determined by secondary metabolites including aroma compounds. Therefore, this thesis project aims to provide novel insights in the effect of near-zero growth rates on the production of aroma compounds by dairy lactic acid bacteria and the stability and copy number of plasmids they contain. Moreover, novel opportunities for optimising aroma formation by lactic acid bacteria at near-zero growth rates are provided.

\section{Outline of this thesis}

This thesis focusses on the physiological and metabolic adaptions of dairy LAB towards near-zero growth rates which could help to develop a production system for aroma compounds that are typically formed during cheese ripening. L. lactis biovar diacetylactis and Lc. mesenteroides were selected as study organisms because these bacteria are considered to be the main aroma producers during ripening of Dutch-type cheeses. The two strains used in the studies are L. lactis subsp. lactis biovar diacetylactis FM03P and Lc. mesenteroides FM06, which were isolated from Danish 10-week-old 45+ Sams $\varnothing$ cheese.

Chapter 2 describes the genomic content of the two selected dairy strains. Genome sequencing revealed that both strains harboured multiple plasmids carrying genes encoding dairy-related functions signifying the adaptation to the dairy environment.

Chapter 3 describes the plasmidome of L. lactis FM03P, containing 12 plasmids, in detail and provides analysis of the putative biological functions of the genes. This analysis revealed many plasmid-encoded dairy functions such as lactose metabolism, citrate uptake, peptide degradation and uptake, exopolysaccharide production, metal uptake, bacteriophage resistance and stress resistance. Plasmid-cured variants were made to confirm some of the putative functions and demonstrate the impact of the plasmids on the growth performance. 
Citrate metabolism is highly relevant for aroma formation by L. lactis biovar diacetylactis, but there still remain some disputes about the regulation mechanisms of citrate uptake in L. lactis. To solve this, regulation of citrate uptake by CitP has been systematically studied in chapter 4 as function of the $\mathrm{pH}$, nutrient limitation and the presence of citrate. Regulation was analysed at four levels: plasmid copy number, citP transcription, citP mRNA processing and citrate utilisation capacity. This provided novel insights in the regulation of citrate uptake that could help to optimise aroma formation from citrate.

Chapter 5 compares aroma formation by L. lactis in a milli-cheese model system with aroma formation in liquid cultures using batch and retentostat cultivation in three different media (chemically defined medium, hydrolysed micellar casein isolate and milk). Qualitative and quantitative comparisons showed that aroma formation during cheese ripening was best resembled using retentostat cultivation despite the use of a chemically defined medium revealing that near-zero growth rates are required to produce aroma compounds similar to those produced during cheese ripening.

The effect of near-zero growth rates on the physiology and aroma formation capacity of $L$. lactis was further explored in chapter 6 . In the retentostat cultures, a large fraction of the cells became viable but not culturable as also found for cheese. Quantitative models were constructed to describe the growth in the retentostat cultures revealing that the maintenance requirements of this dairy $L$. lactis strain is dependent on the growth rate, in contrast to what was found previously for a plant-associated L. lactis strain. Finally, changes in aroma formation were compared with aroma formation during cheese ripening.

Chapter $\mathbf{7}$ describes the physiological and metabolic adaptations of $L c$. mesenteroides FM06 towards near-zero growth rates, which were very similar to the adaptations of $L$. lactis described in chapter 6 . Furthermore, both LAB strains were combined in retentostat co-cultures to asses if this could enhance the formation of aroma compounds and the aroma complexity. Quantitative models were constructed that could accurately predict growth in the co-cultures providing opportunities to select proper combinations of microorganisms for retentostat cocultivation.

Because plasmids in LAB carry important dairy functions, the copy numbers of five of the plasmids of $L$. lactis FM03P were monitored during chemostat and retentostat cultivations to determine if the extreme nutrient limitation affected plasmid maintenance (chapter 8). Plasmids copy numbers were remarkable stable throughout the cultivations indicating that plasmid replication was strictly controlled. 
Although retentostat cultivation appeared to be a promising tool to produce aroma compounds, it has disadvantages in terms of time-dependency and reproducibility. Therefore, $L$. lactis was grown in a partial cell recycling chemostat to demonstrate its potential as aroma production system under truly static conditions (chapter 9).

Finally, in chapter $\mathbf{1 0}$ the results of the experimental chapters have been combined with relevant literature to provide perspectives for future research and application opportunities. 


\section{References}

Ainsworth, S., Stockdale, S., Bottacini, F., Mahony, J. and van Sinderen, D. (2014). The Lactococcus lactis plasmidome: much learnt, yet still lots to discover. FEMS Microbiol. Rev. 38(5): 1066-1088.

Alting, A. C., Engels, W. J. M., van Schalkwijk, S. and Exterkate, F. A. (1995). Purification and characterization of cystathionine $\beta$-lyase from Lactococcus lactis subsp. cremoris $\mathrm{B} 78$ and its possible role in flavor development in cheese. Appl. Environ. Microbiol. 61(11): 4037-4042.

Bandell, M., Lhotte, M. E., Marty-Teysset, C., Veyrat, A., Prévost, H., Dartois, V., Diviès, C., Konings, W. N. and Lolkema, J. S. (1998). Mechanism of the citrate transporters in carbohydrate and citrate cometabolism in Lactococcus and Leuconostoc species. Appl. Environ. Microbiol. 64(5): 1594-1600.

Bonnarme, P., Amarita, F., Chambellon, E., Semon, E., Spinnler, H. E. and Yvon, M. (2004). Methylthioacetaldehyde, a possible intermediate metabolite for the production of volatile sulphur compounds from L-methionine by Lactococcus lactis. FEMS Microbiol. Lett. 236(1): 85-90.

Chambellon, E., Rijnen, L., Lorquet, F., Gitton, C., van Hylckama Vlieg, J. E., Wouters, J. A. and Yvon, M. (2009). The D-2-hydroxyacid dehydrogenase incorrectly annotated PanE is the sole reduction system for branched-chain 2-keto acids in Lactococcus lactis. J. Bacteriol. 191(3): 873-881.

Chapot-Chartier, M. P., Deniel, C., Rousseau, M., Vassal, L. and Gripon, J.-C. (1994). Autolysis of two strains of Lactococcus lactis during cheese ripening. Int. Dairy J. 4(3): 251-269.

Choi, Y.-O. and Ahn, C. (1997). Plasmid-associated bacteriocin production by Leuconostoc sp. LAB145-3A isolated from Kimchi. J. Microbiol. Biotechnol. 7(6): 409-416.

Collins, Y. F., McSweeney, P. L. H. and Wilkinson, M. G. (2003). Lipolysis and free fatty acid catabolism in cheese: a review of current knowledge. Int. Dairy J. 13(11): 841-866.

Davey, G. P. (1984). Plasmid associated with diplococcin production in Streptococcus. Appl. Environ. Microbiol. 48(4): 895-896.

David, S., Stevens, H., Van Riel, M., Simons, G. and De Vos, W. (1992). Leuconostoc lactis beta-galactosidase is encoded by two overlapping genes. J. Bacteriol. 174(13): 4475-4481.

de Felipe, F. L., Magni, C., de Mendoza, D. and López, P. (1995). Citrate utilization gene cluster of the Lactococcus lactis biovar diacetylactis: organization and regulation of expression. Molecular and General Genetics MGG 246(5): 590-599.

de Vos, W. M., Vos, P., de Haard, H. and Boerrigter, I. (1989). Cloning and expression of the Lactococcus lactis subsp. cremoris SK11 gene encoding an extracellular serine proteinase. Gene 85(1): 169-176.

de Vos, W. M., Boerrigter, I., van Rooyen, R. J., Reiche, B. and Hengstenberg, W. (1990). Characterization of the lactose-specific enzymes of the phosphotransferase system in Lactococcus lactis. J. Biol. Chem. 265(36): 22554-22560.

Delorme, C., J.J., G., Ehrlich, S. D. and Renault, P. (1993). Gene inactivation in Lactococcus lactis: histidine biosynthesis. J. Bacteriol. 175(14): 4391-4399.

Ercan, O., Bisschops, M. M. M., Overkamp, W., Jorgensen, T. R., Ram, A. F., Smid, E. J., Pronk, J. T., Kuipers, O. P., Daran-Lapujade, P. and Kleerebezem, M. (2015). Physiological and transcriptional responses of different industrial microbes at near-zero specific growth rates. Appl. Environ. Microbiol. 81(17): 5662-5670.

Erkus, O., de Jager, V. C. L., Spus, M., van Alen-Boerrigter, I. J., van Rijswijck, I. M. H., Hazelwood, L., Janssen, P. W. M., van Hijum, S. A. F. T., Kleerebezem, M. and Smid, E. J. (2013). Multifactorial diversity sustains microbial community stability. ISME J 7(11): 2126-2136.

Fallico, V., McAuliffe, O., Fitzgerald, G. F. and Ross, R. P. (2011). Plasmids of raw milk cheese isolate Lactococcus lactis subsp. lactis biovar diacetylactis DPC3901 suggest a plant-based origin for the strain. Appl. Environ. Microbiol. 77(18): 6451-6462. 
Fernández, L., Beerthuyzen, M. M., Brown, J., Siezen, R. J., Coolbear, T., Holland, R. and Kuipers, O. P. (2000). Cloning, characterization, controlled overexpression, and inactivation of the major tributyrin esterase gene of Lactococcus lactis. Appl. Environ. Microbiol. 66(4): 1360-1368.

Forde, A. and Fitzgerald, G. F. (1999). Analysis of exopolysaccharide (EPS) production mediated by the bacteriophage adsorption blocking plasmid, pCI658, isolated from Lactococcus lactis ssp. cremoris HO2. Int. Dairy J. 9(7): 465-472.

Fox, P. F., Guinee, T. P., Cogan, T. M. and McSweeney, P. L. H. (2000). Fundamentals of cheese science (2nd ed.). New York: Springer.

Fox, P. F., Guinee, T. P., Cogan, T. M. and McSweeney, P. L. H. (2017). Biochemistry of cheese ripening. In Fox, P. F., Guinee, T. P., Cogan, T. M. and McSweeney, P. L. $\mathrm{H}$. (Eds.), Fundamentals of cheese science (pp. 391-442). Boston, MA: Springer.

Fox, P. F. and McSweeney, P. L. H. (2017). Cheese: an overview. In McSweeney, P. L. H., Fox, P. F., Cotter, P. and Everett, D. W. (Eds.), Cheese: chemistry, physics and microbiology (4th ed., Vol. 1, pp. 5-21). San Diego: Academic Press.

García-Quintáns, N., Magni, C., de Mendoza, D. and López, P. (1998). The citrate transport system of Lactococcus lactis subsp. lactis biovar diacetylactis is induced by acid stress. Appl. Environ. Microbiol. 64(3): 850-857.

García-Quintáns, N., Repizo, G., Martín, M., Magni, C. and López, P. (2008). Activation of the diacetyl/acetoin pathway in Lactococcus lactis subsp. lactis bv. diacetylactis CRL264 by acidic growth. Appl. Environ. Microbiol. 74(7): 1988-1996.

Gasson, M. (1984). Transfer of sucrose fermenting ability, nisin resistance and nisin production into Streptococcus lactis 712. FEMS Microbiol. Lett. 21(1): 7-10.

Godon, J. J., Delorme, C., Bardowski, J., Chopin, M. C., Ehrlich, S. D. and Renault, P. (1993). Gene inactivation in Lactococcus lactis: branched-chain amino acid biosynthesis. J. Bacteriol. 175(14): 4383-4390.

Harmon, K. S. and McKay, L. L. (1987). Restriction enzyme analysis of lactose and bacteriocin plasmids from Streptococcus lactis subsp. diacetylactis WM4 and cloning of BclI fragments coding for bacteriocin production. Appl. Environ. Microbiol. 53(5): 1171-1174.

Holland, R. and Coolbear, T. (1996). Purification of tributyrin esterase from Lactococcus lactis subsp. cremoris E8. J. Dairy Res. 63(1): 131-140.

Holo, H., Nilssen, $\varnothing$. and Nes, I. (1991). Lactococcin A, a new bacteriocin from Lactococcus lactis subsp. cremoris: isolation and characterization of the protein and its gene. J. Bacteriol. 173(12): 3879-3887.

Hugenholtz, J. (1993). Citrate metabolism in lactic acid bacteria. FEMS Microbiol. Rev. 12(1-3): 165-178.

Juillard, V., Le Bars, D., Kunji, E. R., Konings, W. N., Gripon, J. C. and Richard, J. (1995). Oligopeptides are the main source of nitrogen for Lactococcus lactis during growth in milk. Appl. Environ. Microbiol. 61(8): 3024-3030.

Kelleher, P., Bottacini, F., Mahony, J., Kilcawley, K. N. and van Sinderen, D. (2017). Comparative and functional genomics of the Lactococcus lactis taxon; insights into evolution and niche adaptation. BMC Genomics 18(1): 267.

Kempler, G. M. and Mckay, L. L. (1979). Characterization of plasmid deoxyribonucleic acid in Streptococcus lactis subsp. diacetylactis: evidence for plasmid-linked citrate utilization. Appl. Environ. Microbiol. 37(2): 316-323.

Klaenhammer, T. R. and Sanozky, R. B. (1985). Conjugal transfer from Streptococcus lactis ME2 of plasmids encoding phage resistance, nisin resistance and lactosefermenting ability: evidence for a high-frequency conjugative plasmid responsible for abortive infection of virulent bacteriophage. Microbiology 131(6): 1531-1541.

Kunji, E. R. S., Mierau, I., Poolman, B., Konings, W. N., Venema, G. and Kok, J. (1996). Fate of peptides in peptidase mutants of Lactococcus lactis. Mol. Microbiol. 21(1): 123-131.

Leelawatcharamas, V., Chia, L. G., Charoenchai, P., Kunajakr, N., Liu, C.-Q. and Dunn, N. W. (1997). Plasmid-encoded copper resistance in Lactococcus lactis. Biotechnol. Lett. 19(7): 639-643. 
Liu, C.-Q., Khunajakr, N., Chia, L. G., Deng, Y.-M., Charoenchai, P. and Dunn, N. W. (1997). Genetic analysis of regions involved in replication and cadmium resistance of the plasmid pND302 from Lactococcus lactis. Plasmid 38(2): 79-90.

Liu, C.-Q., Charoechai, P., Khunajakr, N., Deng, Y.-M., Widodo and Dunn, N. W. (2002). Genetic and transcriptional analysis of a novel plasmid-encoded copper resistance operon from Lactococcus lactis. Gene 297(1): 241-247.

Martín, M. G., Sender, P. D., Peirú, S., de Mendoza, D. and Magni, C. (2004). Acid inducible transcription of the operon encoding the citrate lyase complex of Lactococcus lactis biovar diacetylactis CRL264. J. Bacteriol. 186(17): 5649-5660.

McGovern, P. E., Zhang, J., Tang, J., Zhang, Z., Hall, G. R., Moreau, R. A., Nuñez, A., Butrym, E. D., Richards, M. P., Wang, C.-s., Cheng, G., Zhao, Z. and Wang, C. (2004). Fermented beverages of pre- and proto-historic China. Proc. Natl. Acad. Sci. U. S. A. 101(51): 17593-17598.

McSweeney, P. L. H. (2004). Biochemistry of cheese ripening. Int. J. Dairy Technol. 57(23): 127-144.

Molimard, P. and Spinnler, H. E. (1996). Review: compounds involved in the flavor of surface mold-ripened cheeses: origins and properties. J. Dairy Sci. 79(2): 169184.

Nardi, M., Renault, P. and Monnet, V. (1997). Duplication of the pepF gene and shuffling of DNA fragments on the lactose plasmid of Lactococcus lactis. J. Bacteriol. 179(13): 4164-4171.

Nierop Groot, M. N. and de Bont, J. A. (1998). Conversion of phenylalanine to benzaldehyde initiated by an aminotransferase in Lactobacillus plantarum. Appl. Environ. Microbiol. 64(8): 3009-3013.

Parente, E., Cogan, T. M. and Powell, I. B. (2017). Starter cultures: General aspects. In McSweeney, P. L. H., Fox, P. F., Cotter, P. and Everett, D. W. (Eds.), Cheese, Physics and microbiologu (4th ed., Vol. 1, pp. 201-226). San Diego: Academic Press.

Pasteur, L. (1857a). Mémoire sur la fermentation applée lactique. Comptes Rendus de I'Académie des Sciences 45: 913-916.

Pasteur, L. (1857b). Mémoire sur la fermentation alcoolique. Comptes rendus de l'Académie des Sciences 45: 1032-1036.

Rademaker, J. L. W., Herbet, H., Starrenburg, M. J. C., Naser, S. M., Gevers, D., Kelly, W. J., Hugenholtz, J., Swings, J. and van Hylckama Vlieg, J. E. T. (2007). Diversity analysis of dairy and nondairy Lactococcus lactis isolates, using a novel multilocus sequence analysis scheme and (GTG) 5-PCR fingerprinting. Appl. Environ. Microbiol. 73(22): 7128-7137.

Salque, M., Bogucki, P. I., Pyzel, J., Sobkowiak-Tabaka, I., Grygiel, R., Szmyt, M. and Evershed, R. P. (2012). Earliest evidence for cheese making in the sixth millennium BC in northern Europe. 493: 522.

Scherwitz, K. M., Baldwin, K. A. and McKAY, L. L. (1983). Plasmid linkage of a bacteriocinlike substance in Streptococcus lactis subsp. diacetylactis strain WM4:

transferability to Streptococcus lactis. Appl. Environ. Microbiol. 45(5): 1506-1512.

Schouler, C., Clier, F., Lerayer, A. L., Ehrlich, S. D. and Chopin, M.-C. (1998). A type IC restriction-modification system in Lactococcus lactis. J. Bacteriol. 180(2): 407411.

Sender, P. D., Martín, M. G., Peirú, S. and Magni, C. (2004). Characterization of an oxaloacetate decarboxylase that belongs to the malic enzyme family. FEBS Lett. 570(1-3): 217-222.

Siezen, R. J., Renckens, B., van Swam, I., Peters, S., van Kranenburg, R., Kleerebezem, M. and de Vos, W. M. (2005). Complete sequences of four plasmids of Lactococcus lactis subsp. cremoris SK11 reveal extensive adaptation to the dairy environment. Appl. Environ. Microbiol. 71(12): 8371-8382.

Smid, E. J., Poolman, B. and Konings, W. N. (1991). Casein utilization by lactococci. Appl. Environ. Microbiol. 57(9): 2447-2452.

Smid, E. J. and Kleerebezem, M. (2014). Production of aroma compounds in lactic fermentations. Annu. Rev. Food Sci. Technol. 5(1): 313-326. 
Smid, E. J., Erkus, O., Spus, M., Wolkers-Rooijackers, J. C., Alexeeva, S. and Kleerebezem, M. (2014). Functional implications of the microbial community structure of undefined mesophilic starter cultures. Microb. Cell Fact. 13(1): S2.

Smit, G., Smit, B. A. and Engels, W. J. M. (2005). Flavour formation by lactic acid bacteria and biochemical flavour profiling of cheese products. FEMS Microbiol. Rev. 29(3): 591-610.

Snoep, J. L., Demattos, M. J. T., Starrenburg, M. J. C. and Hugenholtz, J. (1992). Isolation, characterization, and physiological role of the pyruvate dehydrogenase complex and alpha-acetolactate synthase of Lactococcus lactis subsp. lactis bv. diacetylactis. J. Bacteriol. 174(14): 4838-4841.

Sørhaug, T. and Ordal, Z. J. (1974). Cell-bound lipase and esterase of Brevibacterium linens. Appl. Microbiol. 27(3): 607-608.

Spus, M., Liu, H., Wels, M., Abee, T. and Smid, E. J. (2017). Isolation and characterization of Lactobacillus helveticus DSM 20075 variants with improved autolytic capacity. Int. J. Food Microbiol. 241: 173-180.

Tanous, C., Chambellon, E., Sepulchre, A.-M. and Yvon, M. (2005). The gene encoding the glutamate dehydrogenase in Lactococcus lactis is part of a remnant $\operatorname{Tn} 3$ transposon carried by a large plasmid. J. Bacteriol. 187(14): 5019-5022.

Tynkkynen, S., Buist, G., Kunji, E., Kok, J., Poolman, B., Venema, G. and Haandrikman, A. (1993). Genetic and biochemical characterization of the oligopeptide transport system of Lactococcus lactis. J. Bacteriol. 175(23): 7523-7532.

van Kranenburg, R., Marugg, J. D., Van Swam, I. I., Willem, N. J. and De Vos, W. M. (1997). Molecular characterization of the plasmid-encoded eps gene cluster essential for exopolysaccharide biosynthesis in Lactococcus lactis. Mol. Microbiol. 24(2): 387-397.

van Kranenburg, R., Kleerebezem, M. and de Vos, W. M. (2000). Nucleotide sequence analysis of the lactococcal EPS plasmid pNZ4000. Plasmid 43(2): 130-136.

van Rooijen, R. J. and de Vos, W. M. (1990). Molecular cloning, transcriptional analysis, and nucleotide sequence of $l a c R$, a gene encoding the repressor of the lactose phosphotransferase system of Lactococcus lactis. J. Biol. Chem. 265(30): 1849918503.

van Rooijen, R. J., van Schalkwijk, S. and de Vos, W. M. (1991). Molecular cloning, characterization, and nucleotide sequence of the tagatose 6-phosphate pathway gene cluster of the lactose operon of Lactococcus lactis. J. Biol. Chem. 266(11): 7176-7181.

Vaughan, E. E., David, S. and de Vos, W. M. (1996). The lactose transporter in Leuconostoc lactis is a new member of the LacS subfamily of galactoside-pentosehexuronide translocators. Appl. Environ. Microbiol. 62(5): 1574-1582.

Vos, P., van Asseldonk, M., Van Jeveren, F., Siezen, R., Simons, G. and De Vos, W. (1989). A maturation protein is essential for production of active forms of Lactococcus lactis SK11 serine proteinase located in or secreted from the cell envelope. J. Bacteriol. 171(5): 2795-2802.

Wang, J., Liu, L., Ball, T., Yu, L., Li, Y. and Xing, F. (2016). Revealing a 5,000-y-old beer recipe in China. Proc. Natl. Acad. Sci. 113(23): 6444-6448.

Yu, W., Gillies, K., Kondo, J. K., Broadbent, J. R. and McKay, L. L. (1996). Loss of plasmidmediated oligopeptide transport system in lactococci: another reason for slow milk coagulation. Plasmid 35(3): 145-155.

Yvon, M. and Rijnen, L. (2001). Cheese flavour formation by amino acid catabolism. Int. Dairy J. 11(4): 185-201. 


\section{Chapter 2}

Complete genome sequences of Lactococcus lactis subsp. lactis bv diacetylactis FM03 and Leuconostoc mesenteroides FM06 isolated from cheese

Oscar van Mastrigt, Tjakko Abee \& Eddy J. Smid

Published in Genome Announcements, July 2017, 5(28):e00633-17 Published online: $13 \mathrm{Jul} 2017$, doi: 10.1128/genomeA.00633-17 


\section{Abstract}

Here, the genome sequences of Lactococcus lactis subsp. lactis bv diacetylactis FM03 and Leuconostoc mesenteroides FM06, both isolated from cheese, are presented. FM03 and FM06 contain 7 and 3 plasmids, respectively, that carry genes encoding functions important for growth and survival in dairy fermentations. 


\section{Genome announcement}

Leuconostoc mesenteroides and Lactococcus lactis subsp. lactis bv diacetylactis are two species of lactic acid bacteria present in starter cultures for cheese production. Compared to $L$. lactis subsp. cremoris strains, LC. mesenteroides and L. lactis subsp. lactis strains show better survival during cheese ripening and thus provide an important contribution to the flavour development (Erkus et al., 2013). Moreover, these two species of lactic acid bacteria also have the ability to utilise citrate, enhancing the production of the buttery aroma compounds acetoin and diacetyl (Starrenburg and Hugenholtz, 1991). In L. lactis subsp. lactis the gene for citrate transport is located on a plasmid (Kempler and Mckay, 1979), showing the importance of plasmids for lactococcal performance in dairy applications. Another trait regularly carried by plasmids is the resistance to bacteriophages (Allison and Klaenhammer, 1998).

Lactococcus lactis subsp. lactis bv diacetylactis FM03 and Leuconostoc mesenteroides FM06 were isolated from 10-week-old Sams $\varnothing$ cheese. Their genomes were sequenced using an Illumina HiSeq 2500 and a PacBio RS instrument. Respectively, 6.4 and 4.3 million quality-filtered paired-end Illumina sequence reads of $100 \mathrm{bp}$ were de novo assembled into contig sequences using CLC Genomics Workbench version 7.0.4 (CLC Bio, Aarhus, Denmark). The contigs were linked and placed into scaffolds based on alignment of the PacBio reads (total, $167 \mathrm{Mbp}$ and $493 \mathrm{Mbp}$, respectively). This resulted in 8 and 4 scaffolds for FM03 and FM06, respectively. Three out of twelve scaffolds were already circular. The remaining scaffolds were closed with PCR, followed by Sanger sequencing. The complete sequence of L. lactis FM03 was annotated using RAST (Aziz et al., 2008) and manually curated. The sequence of Lc. mesenteroides FM06 was annotated with the NCBI Prokaryotic genome annotation pipeline (Tatusova et al., 2016).

The genome sequence of $L$. lactis FM03 contains a chromosome of $2.43 \mathrm{Mbp}$ with a $\mathrm{G}+\mathrm{C}$ content of $35.3 \%$ and 7 plasmids with sizes of $3.4,4.2,7.5,8.3,12.0$, 15.2 , and $30.3 \mathrm{kbp}$ and $\mathrm{G}+\mathrm{C}$ contents of $33.8,35.6,33.6,34.8,33.5,34.1$, and $35.2 \%$, respectively. The plasmids contained genes involved in citrate utilisation (citQRP), resistance to bacteriophages (five different specificity subunits of a type I restriction/modification system) and resistance to several stresses. In most plasmids, transposons were also identified.

The genome sequence of Lc. mesenteroides FM06 contains a chromosomal sequence of $1.89 \mathrm{Mbp}$ with a $\mathrm{G}+\mathrm{C}$ content of $38.0 \%$, and 3 plasmid sequences with sizes of $8.8,15.3$ and $31.1 \mathrm{kbp}$ and $\mathrm{G}+\mathrm{C}$ contents of $35.2,39.2$, and $36.0 \%$, 
respectively. The plasmids contained genes involved in metal transport, lactose utilisation, and resistance to bacteriophages. Interestingly, genes for lactose utilisation (lactose phosphotransferase system (PTS) encoded by lacS (Vaughan et al., 1996) and $\beta$-galactosidase encoded by lacLM (David et al., 1992)) were carried by two different plasmids.

Further investigations into the genomes of these lactic acid bacteria may provide more insight into the role of plasmids in growth and survival in dairy fermentations.

\section{Accession numbers}

The genome sequence of Lactococcus lactis FM03 and Leuconostoc mesenteroides FM06 have been deposited at GenBank under the accession numbers CP020604 to CP020611 and CP020731 to CP020734, respectively.

\section{Acknowledgement}

This work was financially supported by Arla Foods, Aarhus, Denmark. 


\section{References}

Allison, G. E. and Klaenhammer, T. R. (1998). Phage resistance mechanisms in lactic acid bacteria. Int. Dairy J. 8(3): 207-226.

Aziz, R. K., Bartels, D., Best, A. A., DeJongh, M., Disz, T., Edwards, R. A., Formsma, K., Gerdes, S., Glass, E. M., Kubal, M., Meyer, F., Olsen, G. J., Olson, R., Osterman, A. L., Overbeek, R. A., McNeil, L. K., Paarmann, D., Paczian, T., Parrello, B., Pusch, G. D., Reich, C., Stevens, R., Vassieva, O., Vonstein, V., Wilke, A. and Zagnitko, O. (2008). The RAST Server: rapid annotations using subsystems technology. BMC Genomics 9: 75.

David, S., Stevens, H., van Riel, M., Simons, G. and de Vos, W. M. (1992). Leuconostoc lactis beta-galactosidase is encoded by two overlapping genes. J. Bacteriol. 174(13): 4475-4481.

Erkus, O., de Jager, V. C. L., Spus, M., van Alen-Boerrigter, I. J., van Rijswijck, I. M. H., Hazelwood, L., Janssen, P. W. M., van Hijum, S. A. F. T., Kleerebezem, M. and Smid, E. J. (2013). Multifactorial diversity sustains microbial community stability. ISME J 7(11): 2126-2136.

Kempler, G. M. and Mckay, L. L. (1979). Characterization of plasmid deoxyribonucleic acid in Streptococcus lactis subsp. diacetylactis: evidence for plasmid-linked citrate utilization. Appl. Environ. Microbiol. 37(2): 316-323.

Starrenburg, M. J. and Hugenholtz, J. (1991). Citrate fermentation by Lactococcus and Leuconostoc spp. Appl. Environ. Microbiol. 57(12): 3535-3540.

Tatusova, T., DiCuccio, M., Badretdin, A., Chetvernin, V., Nawrocki, E. P., Zaslavsky, L., Lomsadze, A., Pruitt, K., Borodovsky, M. and Ostell, J. (2016). NCBI prokaryotic genome annotation pipeline. Nucleic Acids Res. 44(14): 6614-6624.

Vaughan, E. E., David, S. and de Vos, W. M. (1996). The lactose transporter in Leuconostoc lactis is a new member of the LacS subfamily of galactoside-pentose-hexuronide translocators. Appl. Environ. Microbiol. 62(5): 1574-1582. 



\section{Chapter 3}

Large plasmidome of dairy Lactococcus lactis subsp. lactis biovar diacetylactis FM03P encodes technological functions and appears highly unstable

Oscar van Mastrigt, Elisa Di Stefano, Sylviani Hartono, Tjakko Abee \& Eddy J. Smid 


\section{Abstract}

Important industrial traits have been linked to plasmids in Lactococcus lactis. The dairy isolate $L$. lactis subsp. lactis biovar diacetylactis FM03P was sequenced revealing the biggest plasmidome of all completely sequenced and published $L$. lactis strains up till now. The 12 plasmids that were identified are: pLd1 $(8,277$ bp), pLd2 (15,218 bp), pLd3 (4,242 bp), pLd4 (12,005 bp), pLd5 (7,521 bp), pLd6 (3,363 bp), pLd7 (30,274 bp), pLd8 (47,015 bp), pLd9 (15,313 bp), pLd10 $(39,563 \mathrm{bp}), \mathrm{pLd} 11(9,833 \mathrm{bp})$ and pLd12 (3,321 bp). Structural analysis of the repB promoters and the RepB proteins showed that eleven of the plasmids replicate via the theta-type mechanism, while only plasmid pLd3 replicates via a rolling-circle replication mechanism. Plasmids pLd2, pLd7 and pLd10 contain a highly similar operon involved in mobilisation of the plasmids. Examination of the twelve plasmids of $L$. lactis FM03P showed that 10 of the plasmids carry putative genes known to be important for growth and survival in the dairy environment. These genes encode technological functions such as lactose utilisation (lacRlacABCDFEGX), citrate uptake (citQRP), peptide degradation (pepO and $p e p E$ ) and oligopeptide uptake (oppDFBCA), uptake of magnesium and manganese (2 $\mathrm{mntH}$, corA), exopolysaccharides production (eps operon), bacteriophage resistance ( 1 $h s d M, 1 h s d R$ and 7 different hsdS genes of a type I restriction-modification system, an operon of three genes encoding a putative type II restrictionmodification system and an abortive infection gene) and stress resistance ( 2 uspA, $\operatorname{csp} C$ and $\operatorname{cad}(A)$. Acquisition of these plasmids most likely facilitated the adaptation of the recipient strain to the dairy environment. Some plasmids were already lost during a single propagation step signifying their instability in the absence of a selective pressure and demonstrating that propagation should be minimised when studying dairy isolates of $L$. lactis. 


\section{Introduction}

Lactococcus lactis is a lactic acid bacterium which is extensively used in food fermentation processes. It is one of the main species used in starter cultures for the production of fermented dairy products, such as cheese, quark, cottage cheese and sour cream (Cavanagh et al., 2015, Leroy and De Vuyst, 2004). L. lactis is naturally present on plants (Klijn et al., 1995, Nomura et al., 2006) and it is proposed that dairy strains have evolved from plant-associated strains transferred to milk via cattle (Cavanagh et al., 2015, Fallico et al., 2011, Kelly et al., 2010, Passerini et al., 2010, Salama et al., 1995, Sandine et al., 1972, Siezen et al., 2008). L. lactis has adapted to the dairy environment by the acquisition of important traits required for the growth on milk, such as lactose catabolism, proteinase activity, citrate utilisation and bacteriophage resistance. Analysis of genomes of $L$. lactis has shown that these traits are often encoded by genes located on plasmids (Mills et al., 2006).

Plasmids are mobile, self-replicating extrachromosomal DNA molecules which can be lost and acquired in response to changing environmental conditions. This behaviour facilitates their distribution among bacteria occupying the same ecological niche. Depending on the environmental conditions, plasmids could have beneficial or adverse effects for the recipient strain. They could give the bacteria the ability to grow better on particular nutrients or survive better under harsh conditions, but at the same time they can be a metabolic burden by either replication of the plasmids or by expression of the plasmid-encoded genes (Kobayashi et al., 2002).

In this study, the complete nucleotide sequences of twelve plasmids of the dairy isolate of $L$. lactis subsp. lactis biovar diacetylactis FM03P are presented, together with analysis of the putative biological functions that were assigned to them. Plasmid-cured variants were made by protoplast-induced curing to confirm some of the putative functions and to demonstrate the impact of the plasmids on growth.

\section{Materials and methods}

\section{Strain and media}

Lactococcus lactis subsp. lactis biovar diacetylactis FM03P, which has been isolated from 10-week-old Sams $\varnothing$ cheese using Nickels and Leesment medium (Nickels and Leesment, 1964), was used in this study as well as several variants with a different plasmid content (Table 3.1). These variants were made by 
protoplast-induced curing or isolated from cultures of L. lactis FM03P. Phenotypic characterisation of the variants started always with streaking the variants on M17 agar plates (Terzaghi and Sandine, 1975) supplemented with either $0.5 \%(\mathrm{w} / \mathrm{v})$ glucose or lactose. After incubation for 2 days at $30^{\circ} \mathrm{C}$ a single colony was inoculated in $\mathrm{M} 17$ supplemented with $0.5 \%$ lactose or glucose and grown overnight at $30^{\circ} \mathrm{C}$.

Table 3.1: Variants of Lactococcus lactis FM03P with different plasmids contents that were obtained in this study. Variants 47,48 and FM03-V1 were isolated by plating on LM17 agar plates.

\begin{tabular}{llll}
\hline $\begin{array}{l}\text { L. lactis } \\
\text { variant }\end{array}$ & Cured plasmids & Plasmid content & Isolated from \\
\hline $\mathrm{FM} 03 \mathrm{P}$ & - & $\mathrm{pLd} 1,2,3,4,5,6,7,8,9,10,11,12$ & Sams $\varnothing$ cheese \\
3 & $\mathrm{pLd} 4,5,10$ & $\mathrm{pLd} 1,2,3,6,7,8,9,11,12$ & Protoplast-induced curing \\
5 & $\mathrm{pLd} 4,10,11$ & $\mathrm{pLd} 1,2,3,5,6,7,8,9,12$ & Protoplast-induced curing \\
47 & $\mathrm{pLd} 8,11$ & $\mathrm{pLd} 1,2,3,4,5,6,7,9,10,12$ & Overnight culture \\
48 & $\mathrm{pLd} 2,4,5,6,7$ & $\mathrm{pLd} 1,3,8,9,10,11,12$ & Chemostat culture \\
49 & $\mathrm{pLd} 2,3,4,5,6,7$ & $\mathrm{pLd} 1,8,9,10,11,12$ & Protoplast-induced curing \\
50 & $\mathrm{pLd} 4,5,6,7$ & $\mathrm{pLd} 1,2,3,8,9,10,11,12$ & Protoplast-induced curing \\
51 & $\mathrm{pLd} 4,5,6,7,8,9,11,12$ & $\mathrm{pLd} 1,2,3,10$ & Protoplast-induced curing \\
63 & $\mathrm{pLd} 3,4,8,11,12$ & $\mathrm{pLd} 1,2,5,6,7,9,10$ & Protoplast-induced curing \\
FM03-V1 & $\mathrm{pLd7}$ & $\mathrm{pLd} 1,2,3,4,5,6,8,9,10,11,12$ & Chemostat culture \\
\hline
\end{tabular}

\section{Whole genome sequencing}

\section{Sequencing of FM03P}

L. lactis FM03P was plated on M17 agar plates supplemented with $0.5 \%$ lactose (LM17) and incubated for 2 days at $30^{\circ} \mathrm{C}$. A single colony was inoculated in LM17 broth and incubated overnight at $30^{\circ} \mathrm{C}$. Subsequently, genomic DNA was extracted using the Wizard $^{\circledR}$ genomic DNA purification kit (Promega, USA), sequenced using an Illumina HiSeq 2500 and a PacBio RS instrument, do novo assembled into contigs and scaffolds and closed by PCR and Sanger sequencing. A more detailed explanation of the method can be found in the genome announcement of $L$. lactis subsp. lactis biovar diacetylactis FM03 (van Mastrigt et al., 2017). In addition to the complete chromosomal sequence, 7 plasmids were found, which were defined as complete circular contigs with a origin of replication and which could be targeted in a PCR.

Sequencing of FM03-V1 and the plasmids pLd8, pLd9, pLd10, pLd11 and pLd12

L. lactis FM03-V1, a single colony isolate of a culture of FM03P, was pre-cultured as described above. Subsequently, genomic DNA was extracted using the DNeasy Blood \& Tissue kit (Qiagen, Germany) according to the manufacturer's procedure, sequenced using an Illumina HiSeq 2500 instrument (total 2.3 million quality- 
filtered paired-end reads and average coverage of 211 times) and de novo assembled into contigs using VelvetOptimiser 1.1.0 ( $\mathrm{k}$-mer of 83). The contig sequences were compared to the obtained sequence of $L$. lactis FM03P, of which only plasmids pLd1 to pLd7 were known at that time. Unmapped contigs with a high coverage were further assembled into scaffolds and closed by PCR and Sanger sequencing. The obtained complete circular sequences are designated plasmids pLd8, pLd9, pLd10, pLd11 and pLd12.

\section{Protoplast-induced curing}

Protoplasts of $L$. lactis FM03P were prepared in Tris- $\mathrm{HCl}$ magnesium chloride sucrose buffer ( $\mathrm{pH} \mathrm{8.0)} \mathrm{according} \mathrm{to} \mathrm{Fujita} \mathrm{et} \mathrm{al.} \mathrm{(1983)} \mathrm{with} \mathrm{some} \mathrm{modifications.}$ Briefly, L. lactis was grown in LM17, harvested in mid-exponential phase by centrifugation ( $5 \mathrm{~min}, 6000 \times \mathrm{g}$ ), washed twice with $30 \mathrm{mM}$ Tris- $\mathrm{HCl}$ buffer $(\mathrm{pH}$ 8.0) and resuspended in a buffer for the lysozyme treatment to degrade the cell wall (30 mM Tris- $\mathrm{HCl}, 3 \mathrm{mM} \mathrm{MgCl}, 20 \%$ sucrose, $100-1000 \mu \mathrm{g} / \mathrm{ml}$ lysozyme, $\mathrm{pH}$ 8.0). After incubation at $37^{\circ} \mathrm{C}$ for $10,60,120$ or 180 minutes, protoplast were regenerated by plating appropriate dilutions of treated samples on regeneration medium (LM17 agar supplemented with $20 \%$ sucrose). After incubation at $30^{\circ} \mathrm{C}$ for 48 to 72 hours, regenerated colonies were picked and screened for their plasmid content.

\section{Screening for plasmid content}

To determine the plasmid content of the variants, DNA was extracted from bacterial cultures using the DNeasy Blood \& Tissue kit (Qiagen, Germany) according to the manufacturer's procedure excluding the RNAse step. Subsequently, unique sequences of each plasmid were amplified in PCR reactions using specific primer sets (Table 3.2). A primer set targeting the chromosome was used as positive control for the DNA extraction. The PCR reaction mixture of $50 \mu \mathrm{l}$ contained $1 \mu$ l purified genomic DNA, $0.2 \mathrm{mM}$ dNTPs (Thermo Scientific, USA), 0.4 $\mu \mathrm{M}$ of forward and reverse primer, $5 \mu$ of $10 \mathrm{x}$ Taq buffer $+\mathrm{MgCl}_{2}$ (Thermo Scientific, USA) and $2 U$ Taq polymerase (Thermo Scientific, USA). The PCR program started with an initial denaturation cycle at $94^{\circ} \mathrm{C}$ for 5 minutes, followed by 25 cycles of $94^{\circ} \mathrm{C}$ for 30 seconds, $58^{\circ} \mathrm{C}$ for 20 seconds and $72^{\circ} \mathrm{C}$ for 45 seconds and a final extension cycle at $72^{\circ} \mathrm{C}$ for 7 minutes. For plasmids pLd7, pLd8, pLd9, pLd10, pLd11 and pLd12 annealing temperatures were increased to $68,60,62$, 63,63 and $60^{\circ} \mathrm{C}$, respectively. PCR amplicons were examined by gel electrophoresis in $1 \%$ agarose gels stained with $\mathrm{SYBR}^{\circledR}$ Safe DNA gel stain (Thermo Scientific, USA) and visualised under UV light (Uvitec, UK). 
Table 3.2: Primer sequences used to detect plasmids in L. lactis FM03P. Primers were used to determine the plasmid content of different variants and to discriminate variants in competition experiments.

\begin{tabular}{|c|c|c|c|c|}
\hline \multirow{2}{*}{$\begin{array}{l}\text { Target } \\
\text { Chromosome }\end{array}$} & \multicolumn{2}{|c|}{ Sequence $\left(5^{\prime} \rightarrow 3^{\prime}\right)$} & \multirow{2}{*}{$\begin{array}{l}\text { Amplicon } \\
254\end{array}$} & \multirow{2}{*}{$\begin{array}{l}\mathbf{T m} \\
\left({ }^{\circ} \mathbf{C}\right) \\
65.2 \\
65.6\end{array}$} \\
\hline & $\begin{array}{l}\text { Fw } \\
\text { Ry }\end{array}$ & TTAATTCAACCTGGAGACACAGTCTTAG & & \\
\hline \multirow[t]{2}{*}{ pLd1 } & Fw & GCATTGACGGCTGTTGTAAT & 209 & 62.5 \\
\hline & Rv & AGCAGATTCCCGAGGATAAC & & 62.0 \\
\hline \multirow[t]{2}{*}{ pLd2 } & Fw & AATGGGCCGAAGGTTCTATT & 285 & 63.4 \\
\hline & Rv & CAGGAACCGATTCTCCTGTTA & & 62.9 \\
\hline \multirow[t]{2}{*}{ pLd3 } & Fw & CCTCTCGCGTTCCTTGATA & 417 & 62.9 \\
\hline & Rv & CCACGTAAGGGCGATTTAGT & & 62.7 \\
\hline \multirow[t]{2}{*}{ pLd4 } & Fw & GCGGTAACAACATCCGTATC & 508 & 61.8 \\
\hline & $\mathrm{Rv}$ & AGTCAGCCCAAGCGACTAAT & & 62.7 \\
\hline \multirow[t]{2}{*}{ pLd5 } & Fw & AAATACAAGTGTTGAAGGCGTTG & 589 & 63.8 \\
\hline & Rv & АССТTTGTTCTCСАATTTCAGC & & 62.2 \\
\hline \multirow[t]{2}{*}{ pLd6 } & Fw & TAAGTGCAACTAAAAGAAATAATAAAGTGCAA & 200 & 65.1 \\
\hline & Rv & TTGCTGATGATTGTACCAGCTAAAAC & & 65.8 \\
\hline \multirow[t]{2}{*}{ pLd7 } & FW & TGGGCATCTAGATAATCTGACGACATCTGT & 596 & 71.3 \\
\hline & Rv & CGACATTGACTCСССАAАAACCAAAAATGA & & 75.3 \\
\hline \multirow[t]{2}{*}{ pLd8 } & Fw & CCCAGTTGATTTAGAATTAGCTGAAGAATA & 588 & 65.8 \\
\hline & Rv & AGATAGGTTGCATCCAAGATAAATTTTTA & & 65.9 \\
\hline \multirow[t]{2}{*}{ pLd9 } & Fw & TAGTCGCTGGCAAATTTTACAATCA & 323 & 67.0 \\
\hline & $\mathrm{Rv}$ & CTTTGGGGGTTGCTTTAGAATCAAT & & 67.5 \\
\hline \multirow{2}{*}{ pLd10 } & Fw & ACGCTTGAACCCCATCTTGG & 255 & 68.3 \\
\hline & $\mathrm{Rv}$ & TCGTCCCAAACGGTTTACCC & & 67.8 \\
\hline \multirow[t]{2}{*}{ pLd11 } & Fw & TTCAATGAATGGCTCGGAAGAA & 388 & 67.6 \\
\hline & $\mathrm{Rv}$ & TTTCGGCACAGGAGCAACAT & & 67.9 \\
\hline \multirow[t]{2}{*}{ pLd12 } & Fw & GAAAACTAATCTAGTACAATCATCAGCAAACTT & 277 & 65.1 \\
\hline & Rv & ПTाTACGAGTTATATTGTTCTAGTCAGATTCTT & & 64.7 \\
\hline
\end{tabular}

\section{Phenotype testing}

\section{Growth in $L M 17$}

The maximum growth rate of all variants in LM17 was determined with the twofold dilution method in a Bioscreen C as described by Biesta-Peters et al. (2010). Briefly, maximum growth rates were determined with three biological triplicates and two technical duplicates and performing five dilutions per replicate. A single colony was inoculated in LM17 broth and grown overnight at $30^{\circ} \mathrm{C}$. Subsequently, $500 \mu \mathrm{l}$ of the overnight culture was transferred to $9.5 \mathrm{ml}$ fresh LM17 and grown for 3 hours at $30^{\circ} \mathrm{C}$ to have an exponentially growing culture. The exponential culture was diluted $>1000$ times in LM17 medium and two-fold dilutions were made in a 100 -well Honeycomb plate, which was incubated for 24 hours at $30^{\circ} \mathrm{C}$ with measurements of the optical density at $600 \mathrm{~nm}$ every five minutes. Before each measurement, the plate was shaken for 15 seconds. The lowest dilution contained $10^{4} \mathrm{CFU} / \mathrm{ml}$ at the start of the incubation. The time to detect an optical density of 0.2 was determined for each dilution from which the maximum growth 
rate was determined as described by Biesta-Peters et al. (2010). The maximum growth rates correspond to $-1 /$ slope when plotting the natural logarithm of the inoculum concentration versus the time to detection. Representative wells of the highest inoculum concentration were used to compare the growth curves (Fig. 3.6A).

\section{Growth performance experiments}

The growth performance of variants 48 and 50, differing in plasmid pLd2, was assessed by sequential propagation in mixed cultures. The variants were grown overnight in M17 supplemented with $0.5 \%$ glucose (GM17) and mixed in a ratio $1: 1$ based on optical density measurements at $600 \mathrm{~nm}$ in GM17 supplemented with $0.035 \mathrm{mM} \mathrm{CdCl} 2$. Subsequently, the culture was incubated at $30^{\circ} \mathrm{C}$ and propagated every 48 hours at $1 \%$ inoculation level $(100 \mu$ added to $10 \mathrm{ml}$ fresh medium) for 4 times. To determine the ratio of the variants at the end of the propagation ( $\sim 27$ generations), appropriate dilutions of samples were plated on GM17 agar and incubated for 3 days at $30^{\circ} \mathrm{C}$. Subsequently, the plasmid content was determined of approximately 20 single colonies in three steps: i) DNA extraction, ii) PCR, and iii) gel electrophoresis. DNA was extracted from single colonies by a lysis treatment followed by DNA extraction using the InstaGene ${ }^{T M}$ Matrix (Bio-Rad, USA). Colonies were incubated for $30-45$ minutes at $37^{\circ} \mathrm{C}$ in 200 $\mu \mathrm{l}$ lysis buffer consisting of $20 \mathrm{mM}$ Tris- $\mathrm{HCl}, 2 \mathrm{mM}$ EDTA and $1 \mathrm{mg} / \mathrm{ml}$ lysozyme $(\mathrm{pH}$ 8.0). Subsequently, samples were centrifuged at $13800 \times g$ for 2 minutes and washed with phosphate buffer saline. The pellets were then treated with the InstaGene ${ }^{T M}$ Matrix (Bio-Rad, USA) according to the manufacturer's procedure using $100 \mu \mathrm{l}$ matrix instead of $200 \mu \mathrm{l}$. PCR and gel electrophoresis were performed as explained for the screening of the plasmid content. We used pLd2 to distinguish variant 48 and 50 and pLd1 was used as positive control for a successful DNA extraction.

\section{Results and discussion}

Lactococcus lactis subsp. lactis biovar diacetylactis FM03P has been isolated from 10-week-old Sams $\varnothing$ cheese. Its genome has been sequenced using a combination of an Illumina HiSeq2500 and PacBio RS instrument as previously described (van Mastrigt et al., 2017). This revealed the complete sequence of the chromosome and 7 plasmids, designated pLd1 to pLd7. Subsequent next-generation sequencing attempts of $L$. lactis FM03-V1, a single colony isolate of a culture of $L$. lactis FM03P, using only Illumina revealed the sequence of 5 other plasmids, designated pLd8 to pLd12. The different sequencing attempts have been summarised in Supplemental Figure S3.1. Using PCR and analysis by gel 
electrophoresis, we confirmed the presence of all 12 plasmids in parent strain $L$. lactis FM03P (Suppl. Fig. S3.2). The obtained sequences of pLd8 to pLd12 were not present in the raw unassembled PacBio and Illumina reads obtained from strain FM03P demonstrating that these plasmids were already lost during propagation.

The genome of $L$. lactis subsp. lactis biovar diacetylactis FM03P contains a chromosome of $2.43 \mathrm{Mbp}$ with a $\mathrm{G}+\mathrm{C}$ content of $35.3 \%$ and 12 plasmids with sizes of $8.3,15.2,4.2,12.0,7.5,3.4,30.3,47.0,15.3,39.6,9.8$, and $3.3 \mathrm{kbp}$ and $\mathrm{G}+\mathrm{C}$ contents of $34.8,34.1,35.6,33.5,33.6,33.8,35.2,35.3,35.2,34.9,33.0$, and $33.2 \%$, respectively (Fig. 3.1). The obtained plasmid sequences were annotated using RAST (Aziz et al., 2008) after which the annotation was manually curated and analysed in detail including their replication mechanisms and mobilisation properties.

\section{Replication}

Plasmids replicate independently from the chromosome using plasmid-encoded Rep proteins. Plasmids in lactococci replicate using either rolling-circle replication (RCR) or theta-type replication (del Solar et al., 1998). The replication mechanism as well as the sequence of the replication protein and the origin of replication affect the stability of the plasmids, their copy number and their compatibility. RCR plasmids replicate via the synthesis of ssDNA intermediates, and these plasmids are usually small in size, have multiple copies and are incompatible with other RCR plasmids (Ainsworth et al., 2014, Khan, 1997). Sequence homology of the replication initiator protein and the presence of a double-stranded origin of replication (dso) indicated that pLd3 is the only RCR plasmid in L. lactis FM03P. On pLd2 we found a gene encoding a protein that had high similarity to the Nterminal part of RepB which is normally encoded on RCR plasmids of the type pMV158, but the C-terminal end was found to be missing.

Theta plasmids are more common in lactococci and a single bacterial cell can contain multiple theta plasmids (Seegers et al., 1994). Based on the homology of the replication initiator protein and structural motifs in the rep $B$ promoter, it was concluded that all plasmids except pLd3 replicate with a theta-type mechanism (Suppl. Fig. S3.3 and S3.4). On plasmid pLd8 two repB genes are located of which the second replicon (with the repB2 gene) seems to be functional as it is highly similar to the replicons of other plasmids. In contrast, in the promoter of repB1 the inverted repeat IRb is missing and the C-terminus of the predicted RepB1 protein is quite different from the other predicted RepB proteins (including RepB2). Both the repB genes on pLd10 seem to be functional. The DNA sequences 


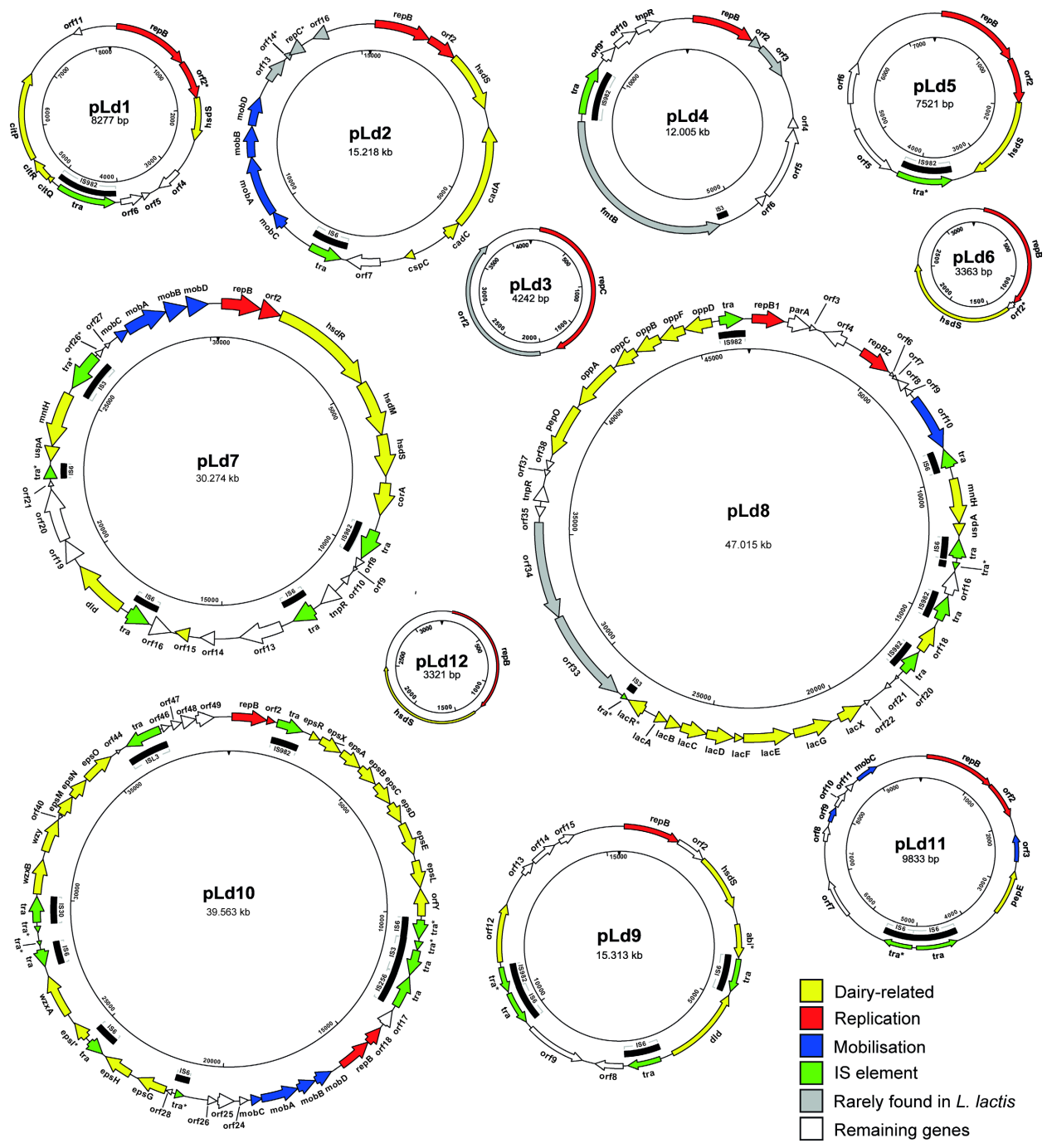

Figure 3.1: Genetic maps of plasmids of L. lactis FM03P. Arrows indicate positions and direction of predicted genes. Colours represent the putative functions of the genes. The name of the genes are indicated and correspond to gene names used in Table 3.4. Pseudogenes are marked with an asterisk. The inner circle corresponds to the nucleotide numbering of the plasmid.

of the repB genes on plasmids pLd2 and pLd9 and their promoters are $100 \%$ identical. This could cause incompatibility of these plasmids and could result in plasmid loss (del Solar et al., 1998, Novick, 1987). This may also explain why plasmid pLd9 was not found in the first sequencing attempt. We also observed spontaneous loss of plasmid pLd2 during continuous chemostat cultivation, while pLd9 was kept (data not shown). 


\section{Mobilisation}

Plasmids can carry conjugation or mobilisation regions that increase their spread in the population via conjugation events. Conjugative plasmids are selftransmissible, while mobilisable plasmids are only transmissible in the presence of additional conjugative functions (Francia et al., 2004). To determine if plasmids were transmissible, the plasmid sequences were searched for known conjugation and mobilisation regions. Plasmids $\mathrm{pLd2}$, pLd7 and pLd10 showed a highly similar (>97\%) operon of 4 genes involved in mobilisation of the plasmid. The DNA sequence of these operons in pLd2, pLd7 and pLd10 is 96,97 and $98 \%$ similar to the mobilisation region of pNZ4000, respectively, which was demonstrated to be functional (van Kranenburg and de Vos, 1998). Plasmid pNZ4000 was found in Lactococcus lactis and carries genes necessary for the production of exopolysaccharides. The oriT sequences, essential for plasmid mobilisation, of pLd2, pLd7 and pLd10 are highly similar to the functional oriT1 sequence of pNZ4000 (Fig. 3.2) and all plasmids carry a mobA gene encoding a relaxase that is involved in nicking at the nic sites of the oriT sequences. Plasmids pLd2, pLd7 and pLd10 also carry mobC which is present but not annotated in pNZ4000. The genes $m o b C$ and $m o b B$, of which the start codon overlaps with the stop codon of mobA, most likely encode accessory proteins for MobA (Perwez and Meyer, 1999, Zhang and Meyer, 1997). The function of mobD, designated mobC in pNZ4000, remains to be elucidated. Recently, the nucleotide sequence of plasmid p229C of L. lactis 229 has been published (Kelleher et al., 2017). This plasmid is remarkably similar to plasmid pLd7 (>99.9\%), while the other plasmids show no or limited similarity. Both strains are isolated from the dairy environment and the mobilisation operon might have increased the transfer rate of this plasmid within the dairy environment. In addition to the mobilisation operons, pLd11 carries 3 genes (orf3, orf9 and $m o b C$ ) encoding proteins that may be involved in mobilisation. Moreover, pLd8 carries a gene encoding a putative conjugal transfer protein (nickase) of the MobA-MobL family. In contrast to the described genes on pLd2, pLd7 and pLd10, these genes are not part of a large mobilisation gene cluster.

Figure 3.2: Multiple sequence alignment of oriT regions of plasmids pLd2, pLd7, pLd10 and pNZ4000 (van Kranenburg and de Vos, 1998). The inverted repeat is shown with dashed arrows and the arrowhead indicates the nic site. The asterisks below indicate identical nucleotides in all four sequences. 


\section{Plasmid-encoded functions}

On the 12 plasmids, we identified and annotated in total 203 putative genes or fragments thereof, which corresponds to $7.4 \%$ of the total number of putative genes present in the entire genome (Table 3.3). Based on homology with other proteins, putative functions were ascribed to $74 \%$ of the plasmid encoded genes. Of all genes $10 \%$ were pseudogenes containing frameshifts, a premature stop codon or truncations. An overview of all the genes in the plasmidome of L. lactis FM03P is given in Figure 3.3 and their putative functions are given in Table 3.4. In the coming sections a selection of genes encoding functions that could enhance growth and survival of the bacteria and/or encode technological properties are described in more detail.

Table 3.3: Summary of plasmid statistics and the putative genes.

\begin{tabular}{llllll}
\hline & & & \multicolumn{3}{c}{ Total no. of: } \\
\cline { 3 - 6 } Plasmid & $\begin{array}{l}\text { Size } \\
(\mathbf{b p )}\end{array}$ & $\begin{array}{l}\text { G+C content } \\
\mathbf{( \% )}\end{array}$ & ORFs & Pseudogenes $^{\mathbf{a}}$ & $\begin{array}{l}\text { Homolog with } \\
\text { known } \\
\text { function }\end{array}$ \\
\hline pLd1 & 8,277 & 33.8 & 11 & $1(0)$ & 7 \\
pLd2 & 15,218 & 35.6 & 16 & $3(0)$ & 14 \\
pLd3 & 4,242 & 33.6 & 2 & 0 & 1 \\
pLd4 & 12,005 & 34.8 & 11 & 1 & 4 \\
pLd5 & 7,521 & 33.5 & 6 & 0 & 5 \\
pLd6 & 3,363 & 34.1 & 3 & 1 & 2 \\
pLd7 & 30,274 & 35.2 & 31 & $3(2)$ & 23 \\
pLd8 & 47,015 & 35.3 & 45 & $3(2)$ & 32 \\
pLd9 & 15,313 & 35.2 & 15 & $2(1)$ & 12 \\
pLd10 & 39,563 & 34.9 & 49 & $5(4)$ & 40 \\
pLd11 & 9,833 & 33.0 & 12 & $1(1)$ & 2 \\
pLd12 & 3,321 & 33.2 & 2 & 0 & 150 \\
Total & 195,945 & & 203 & $20(10)$ & \\
\hline
\end{tabular}

${ }^{a}$ Values in parentheses indicate number of pseudogenes in IS elements 


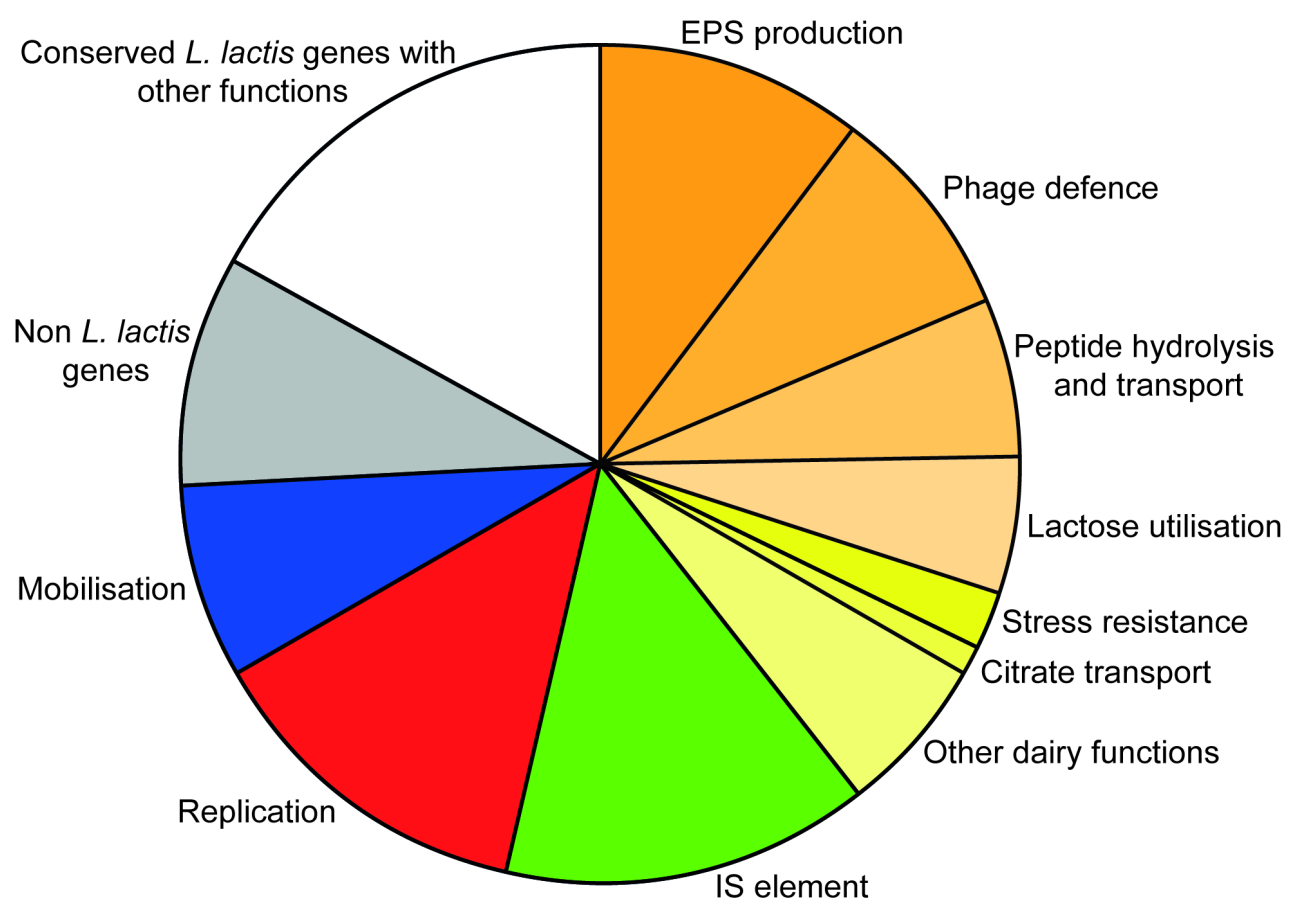

Figure 3.3: Overview of the putative functions of plasmid-encoded genes in L. lactis FM03P. The sum of the sizes of the genes within a category relative to the total size of all plasmid genes was used. The orange to yellow categories represent dairy functions. Other dairy functions include genes for uptake of magnesium and manganese (corA and $m n t H$ ), Dlactate dehydrogenases $(d / d)$, and a C4-dicarboxylate transporter. Non $L$. lactis genes include the genes that are rarely found in L. lactis. Conserved L. lactis genes with other functions consist of all the putative genes which are regularly found in $L$. lactis and encode hypothetical proteins or proteins with functions that did not fit into the other categories. 
Table 3.4: Overview of the putative genes and their putative functions. Putative functions that could be beneficial in the dairy environment are shown in bold. Pseudogenes are indicated with an asterisk. The species that contains the best homolog is given for genes that are rarely found in $L$. lactis.

\begin{tabular}{|c|c|c|c|}
\hline Plasmid & Gene & Putative function & Best homolog found in \\
\hline \multirow[t]{11}{*}{$\mathrm{pLd} 1$} & repB & Replication initiator protein & \\
\hline & orf $2 *$ & Replication-associated protein & \\
\hline & hsdS & $\begin{array}{l}\text { Type I restriction-modification system } \\
\text { specificity subunit } \mathbf{S}\end{array}$ & \\
\hline & orf4 & Hypothetical protein & \\
\hline & orf5 & Hypothetical protein & \\
\hline & orf6 & Hypothetical protein & \\
\hline & tra & Transposase IS982 family & \\
\hline & citQ & Leader peptide CitQ & \\
\hline & citR & Translational regulator & \\
\hline & citP & Citrate transporter & \\
\hline & orf11 & Hypothetical protein & \\
\hline \multirow[t]{16}{*}{$\mathrm{pLd} 2$} & repB & Replication initiator protein & \\
\hline & orf $2 *$ & Replication-associated protein & \\
\hline & hsdS & $\begin{array}{l}\text { Type I restriction-modification system } \\
\text { specificity subunit } \mathbf{S}\end{array}$ & \\
\hline & $\operatorname{cad} A$ & Cadmium-transporting ATPase & \\
\hline & cadC & Cadmium efflux system accessory protein & \\
\hline & $\operatorname{cspC}$ & Cold shock protein & \\
\hline & orf7 & Serine/threonine protein phosphatase & \\
\hline & tra & Transposase IS 6 family & \\
\hline & $m o b c$ & Mobilisation protein & \\
\hline & mobA & Mobilisation protein & \\
\hline & $m o b B$ & Mobilisation protein & \\
\hline & mobD & Mobilisation protein & \\
\hline & orf13 & HXXEE domain-containing protein & Streptococcus thermophilus \\
\hline & orf14* & Xre family transcriptional regulator & Streptococcus thermophilus \\
\hline & repC* & Replication initiator protein & Lactobacillus farciminis \\
\hline & orf16 & Hypothetical protein & Enterococcus faecium \\
\hline \multirow[t]{2}{*}{ pLd3 } & repC & Replication initiator protein & \\
\hline & orf2 & Hypothetical protein & Lactobacillus reuteri \\
\hline \multirow[t]{11}{*}{$\mathrm{pLd4}$} & repB & Replication initiator protein & \\
\hline & orf2 & Hypothetical protein & Enterococcus faecalis \\
\hline & orf3 & Hypothetical protein & Enterococcus faecalis \\
\hline & orf4 & Hypothetical protein & \\
\hline & orf5 & Hypothetical protein & \\
\hline & orf6 & Hypothetical protein & \\
\hline & fmtB & Peptidoglycan-binding protein & Streptococcus thermophilus \\
\hline & tra & Transposase IS982 family & \\
\hline & orfg* & DNA-directed DNA polymerase & \\
\hline & orf10 & Hypothetical protein & \\
\hline & tnpR & Resolvase & \\
\hline \multirow[t]{6}{*}{ pLd5 } & repB & Replication initiator protein & \\
\hline & orf2 & Replication-associated protein & \\
\hline & hsdS & $\begin{array}{l}\text { Type I restriction-modification system } \\
\text { specificity subunit S }\end{array}$ & \\
\hline & tra & Transposase IS982 family & \\
\hline & orf5 & Hypothetical protein & \\
\hline & orf6 & Site-specific integrase & \\
\hline \multirow[t]{3}{*}{$\mathrm{pLd6}$} & repB & Replication initiator protein & \\
\hline & orf $2 *$ & Hypothetical protein & \\
\hline & hsdS & $\begin{array}{l}\text { Type I restriction-modification system } \\
\text { specificity subunit S }\end{array}$ & \\
\hline \multirow[t]{3}{*}{$\mathrm{pLd7}$} & repB & Replication initiator protein & \\
\hline & orf2 & Replication-associated protein & \\
\hline & $h s d R$ & $\begin{array}{l}\text { Type I restriction-modification system } \\
\text { specificity subunit } R\end{array}$ & \\
\hline
\end{tabular}




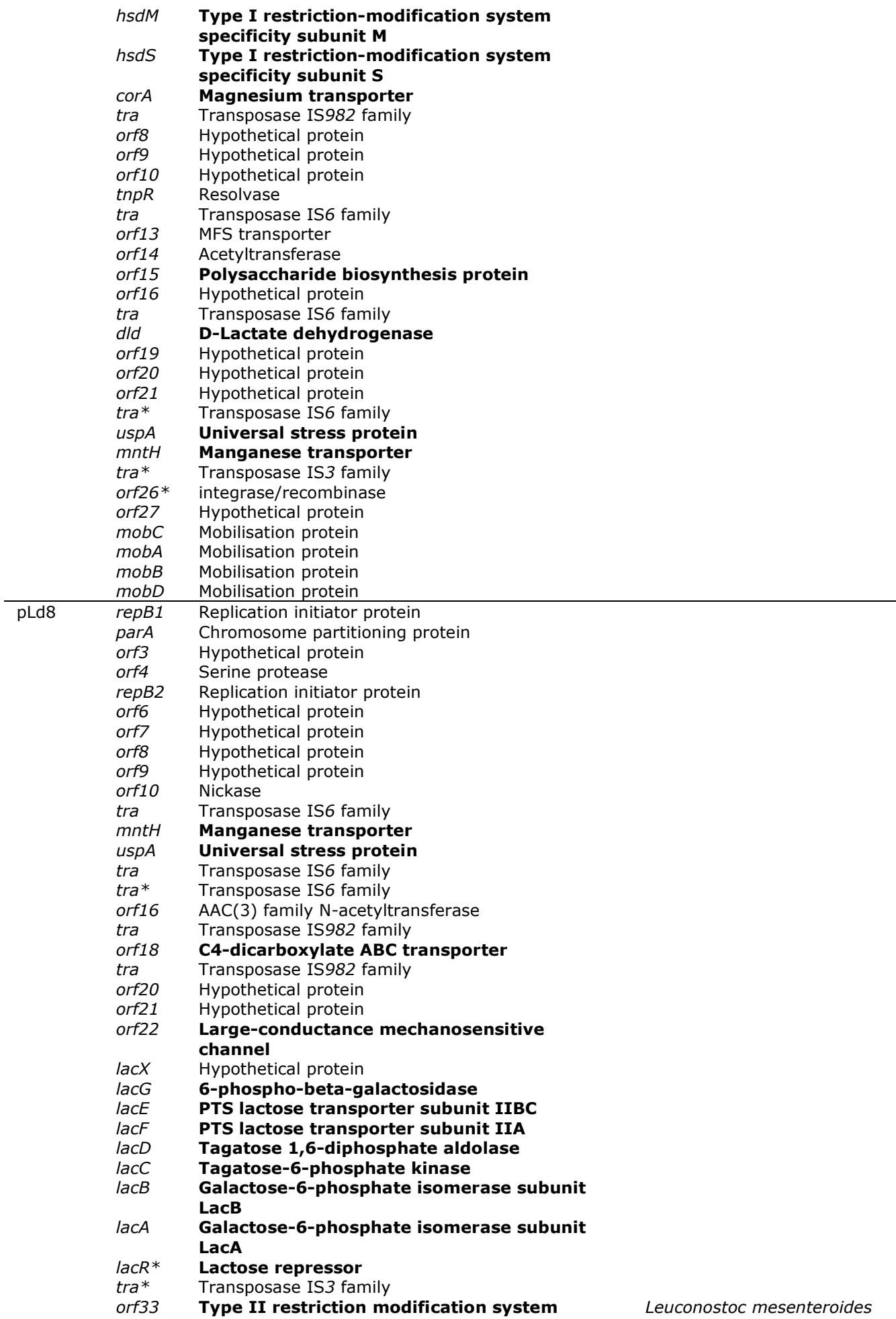




\begin{tabular}{|c|c|c|c|}
\hline & $\begin{array}{l}\text { orf34 } \\
\text { orf35 } \\
\text { tnpR } \\
\text { orf37 } \\
\text { orf38 } \\
\text { pepO } \\
\text { oppA } \\
\text { oppC } \\
\text { oppB } \\
\text { oppF } \\
\text { oppD } \\
\text { tra }\end{array}$ & $\begin{array}{l}\text { Type II restriction modification system } \\
\text { Hypothetical protein } \\
\text { Resolvase } \\
\text { Hypothetical protein } \\
\text { Hypothetical protein } \\
\text { Neutral endopeptidase } \\
\text { Peptide-binding protein } \\
\text { Peptide ABC transporter permease } \\
\text { Peptide ABC transporter permease } \\
\text { Oligopeptide transport ATP-binding protein } \\
\text { Oligopeptide transport ATP-binding protein } \\
\text { Transposase IS982 family }\end{array}$ & Leuconostoc mesenteroides \\
\hline pLd9 & $\begin{array}{l}\text { repB } \\
\text { orf2 } \\
\text { hsdS } \\
\text { abi* } \\
\text { tra } \\
\text { dld } \\
\text { tra } \\
\text { orf8 } \\
\text { orf9 } \\
\text { tra } \\
\text { tra* } \\
\text { orf12 } \\
\text { orf13 } \\
\text { orf14 } \\
\text { orf15 }\end{array}$ & $\begin{array}{l}\text { Replication initiator protein } \\
\text { Hypothetical protein } \\
\text { Type I restriction-modification system } \\
\text { specificity subunit S } \\
\text { Abortive phage resistance protein } \\
\text { Transposase IS6 family } \\
\text { D-lactate dehydrogenase } \\
\text { Transposase IS6 family } \\
\text { Hypothetical protein } \\
\text { MFS transporter } \\
\text { Transposase IS6 family } \\
\text { Transposase IS982 family } \\
\text { Amidohydrolase of peptidase M20 family } \\
\text { Hypothetical protein } \\
\text { Site-specific integrase } \\
\text { Integrase-associated protein }\end{array}$ & \\
\hline pLd10 & $\begin{array}{l}\text { repB1 } \\
\text { orf2 } \\
\text { tra } \\
\text { epsR } \\
\text { epsX } \\
\text { epsA } \\
\text { epsB } \\
\text { epsC } \\
\text { epsD } \\
\text { epsE } \\
\text { epsL } \\
\text { orfY } \\
\text { tra* } \\
\text { tra } \\
\text { tra } \\
\text { tra } \\
\text { orf17 } \\
\text { orf18 } \\
\text { repB2 } \\
\text { mobD } \\
\text { mobB } \\
\text { mobA } \\
\text { mobC } \\
\text { orf24 } \\
\text { orf25 } \\
\text { orf26 } \\
\text { tra* } \\
\text { orf28 } \\
\text { epsG } \\
\text { epsH } \\
\text { tra } \\
\text { epsI* } \\
\text { wzxA } \\
\text { tra } \\
\text { tra* }\end{array}$ & $\begin{array}{l}\text { Replication initiator protein } \\
\text { Replication-associated protein } \\
\text { Transposase IS982 family } \\
\text { XRE family transcriptional regulator } \\
\text { Polysaccharide biosynthesis protein } \\
\text { Tyrosine protein kinase transmembrane } \\
\text { modulator } \\
\text { Tyrosine protein kinase } \\
\text { Tyrosine protein phosphatase } \\
\text { Undecaprenyl-phosphate } \\
\text { galactosephosphotransferase } \\
\text { Group } \mathbf{1} \text { glycosyltransferase } \\
\text { Exopolysaccharide biosynthesis protein } \\
\text { LytR family transcriptional regulator } \\
\text { Transposase IS6 family } \\
\text { Transposase IS3 family } \\
\text { Transposase IS3 family } \\
\text { Transposase IS256 family } \\
\text { XRE family transcriptional regulator } \\
\text { Replication-associated protein } \\
\text { Replication initiator protein } \\
\text { Mobilisation protein } \\
\text { Mobilisation protein } \\
\text { Mobilisation protein } \\
\text { Mobilisation protein } \\
\text { Hypothetical protein } \\
\text { Integrase } \\
\text { Hypothetical protein } \\
\text { Transposase IS6 family } \\
\text { Hypothetical protein } \\
\text { Glycosyltransferase family } \mathbf{2} \text { protein } \\
\text { Glycosyltransferase family } \mathbf{2} \text { protein } \\
\text { Transposase IS6 family } \\
\text { Glycosyltransferase } \\
\text { Flippase } \\
\text { Transposase IS6 family } \\
\text { Transposase }\end{array}$ & $\begin{array}{l}\text { Lactobacillus/ Oenococcus/ } \\
\text { Enterococcus }\end{array}$ \\
\hline
\end{tabular}




\begin{tabular}{|c|c|c|c|}
\hline & tra* & Transposase & Lactobacillus \\
\hline & tra & Transposase IS30 family & Lactobacillus \\
\hline & $w z x B$ & Flippase & Lactobacillus \\
\hline & $w z y$ & Polymerase & Lactobacillus \\
\hline & orf40 & Hypothetical protein & \\
\hline & epsM & Glycosyltransferase family 2 protein & Lactobacillus \\
\hline & epsN & Glycosyltransferase & Lactobacillus \\
\hline & epsO & Glycosyltransferase family 1 protein & Lactobacillus \\
\hline & orf44 & DUF4411 domain-containing protein & Lactobacillus plantarum \\
\hline & tra & Transposase IS $\angle 3$ family & Lactobacillus \\
\hline & orf46 & Hypothetical protein & Lactobacillus \\
\hline & orf47 & Hypothetical protein & \\
\hline & orf48 & Hypothetical protein & \\
\hline & orf49 & Resolvase & \\
\hline \multirow[t]{12}{*}{ pLd11 } & repB & Replication initiator protein & \\
\hline & orf2 & Replication-associated protein & \\
\hline & orf3 & Relaxase/mobilisation nuclease domain protein & \\
\hline & pepE & Peptidase E & \\
\hline & tra & Transposase IS6 family & \\
\hline & tra* & Transposase IS6 family & \\
\hline & orf7 & DUF1919 domain-containing protein & \\
\hline & orf8 & DUF3883 domain-containing protein & \\
\hline & orf9 & Relaxase & \\
\hline & orf10 & Hypothetical protein & \\
\hline & orf11 & Hypothetical protein & \\
\hline & mobC & Mobilisation relaxosome protein & \\
\hline \multirow[t]{2}{*}{ pLd12 } & repB & Replication initiator protein & \\
\hline & hsdS & $\begin{array}{l}\text { Type I restriction-modification system } \\
\text { specificity subunit S }\end{array}$ & \\
\hline
\end{tabular}

\section{Substrate uptake and utilisation}

To thrive in particular environments, microorganisms require specific transporters and metabolic pathways to take up substrates from the environment and use them for growth. Therefore, the presence of genes encoding particular transporters or metabolic enzymes indicate adaptation to a specific environments. To investigate if $L$. lactis FM03P was adapted to the dairy environment, we searched the plasmid sequences for genes involved in utilisation of the main carbon and energy sources found in bovine milk: citrate, lactose and proteins.

Citrate utilisation is characteristic for the biovariety diacetylactis of Lactococcus lactis that contains a plasmid-encoded citQRP operon. In L. lactis FM03P, this operon is located on plasmid pLd1, which is $99 \%$ identical to lactococcal plasmids pCRL1127 and pIL2. The citP gene is encoding a citrate permease enabling the host to take up divalent citrate from the environment (Marty-Teysset et al., 1995). Citrate utilisation results in the generation of a proton motive force in L. lactis (Bandell et al., 1998) and at the same time increases the $\mathrm{pH}$ of the environment (García-Quintáns et al., 1998). Citrate utilisation also has been linked to the production of acetoin and diacetyl. These buttery aromas are important flavour compounds in dairy products. Both citQ and citR are involved in the regulation of expression if citP (de Felipe et al., 1995, Drider et al., 2004). The gene citP is mainly expressed at low pH (around 5.5) when the abundance of divalent citrate 
is maximal (Smith et al., 1992, van Mastrigt et al., 2018), minimising the metabolic burden of maintaining this plasmid.

Genes involved in lactose uptake and utilisation are found on plasmid pLd8, which contains the lacR-lacABCDFEGX operon for lactose uptake via a phosphotransferase system (PTS) and utilisation via the tagatose-6-phosphate pathway. As described for L. lactis IL1403 (Aleksandrzak-Piekarczyk et al., 2005), L. lactis FM03P also contains the chromosomal-encoded Leloir pathway for lactose utilisation. The presence of both pathways in one strain might give this strain a competitive advantage by fast uptake and utilisation of lactose. Interestingly, the LacR protein might not be functional due to a 40 amino acids deletion at the Cterminus, most likely caused by an IS element insertion in the lacR gene. LacR is a transcriptional repressor of the lac operon and deletion of the lac $R$ gene has been shown to increase the activity of the lac promoter both during growth on glucose and lactose (van Rooijen and de Vos, 1990, van Rooijen et al., 1992). Therefore, a non-functional LacR might increase the maximum lactose utilisation rate.

Genes involved in utilisation of proteins, or more specifically oligopeptides, are found on plasmid pLd8, which contains the pepO gene encoding a neutral endopeptidase and the complete oppDFBCA gene cluster encoding the oligopeptide permease (Opp) system (Tynkkynen et al., 1993). All of these genes are also encoded on the chromosome with a high similarity in amino acid sequence (>99\%), except for oppA which only has $87 \%$ similarity to its chromosomal homolog. Plasmid pLd11 carries a pepE gene encoding a putative aspartyl dipeptidase $\mathrm{E}$, which does not have a chromosomal homolog. The opp and pep genes are required, in combination with the extracellular protease PrtP, for utilisation of the milk caseins as nitrogen source (Smid et al., 1991, Tynkkynen et al., 1993). The extracellular protease is often plasmid encoded by prtP and prtM (McKay and Baldwin, 1975). Interestingly, the prtP and prtM genes are not found in $L$. lactis FM03P and this strain does not show a caseinolytic phenotype (data not shown). Presence of the $p e p E$, pepO and oppDFBCA gene cluster and absence of the prtP and prtM genes could give the strain an advantage when growing in combination with a protease-positive $\left(\mathrm{prt}^{+}\right)$strain in milk, in particular at high cell densities (Bachmann et al., 2011). Only the prt $^{+}$strain secretes the protease to hydrolyse the milk caseins, thereby investing energy in production of this protein, while the protease-negative ( $\mathrm{prt}^{-}$) strain can use the peptides generated by the protease without having the burden of protease expression. This combination of $\mathrm{prt}^{+}$and prt- strains is found in many dairy starter cultures, for instance in the Ur starter culture (Erkus et al., 2013). The presence of pepO and opp genes on both the chromosome and plasmid pLd8 could result in faster utilisation of the peptides 
depending on the copy number of the plasmid and the regulation of expression of the pepo and opp genes.

\section{Phage resistance by restriction-modification systems}

An important technological property that is often carried by plasmids is the resistance to bacteriophage infections via the type I restriction-modification system comprising of three subunits. HsdS and HsdM are both necessary for methyltransferase activity, while HsdR is required in addition to the HsdS-HsdM complex for restriction of foreign' DNA that is not methylated (Murray, 2000). The HsdS subunit contains two variable target recognition domains (TRDs) that determine the target sequence specificity of both the restriction and modification activities of the complex (Cowan et al., 1989, Murray, 2000). The variable domains are flanked by conserved regions required for specific associations with the other subunits and for maintaining the relative positions of the two TRDs (Murray, 2000). In L. lactis FM03P the three subunits of this system (HsdR, HsdM and HsdS) are all encoded once on the chromosome. On 7 out of the 12 plasmids other HsdS subunits are found. The protein sequences of all HsdS proteins were aligned with MAFFT (Fig. 3.4). Most of the HsdS proteins contained two variable regions flanked by highly conserved regions as expected. However, the HsdS protein encoded on plasmid pLd1 is not complete. The $h s d S$ gene carried by pLd1 is about half of the size, most likely due to a deletion of 500 to 600 nucleotides. Interestingly, the second variable region of the HsdS proteins of pLd2 and pLd5 are identical and also $97 \%$ similar to pLd7, while the first variable regions do not show significant homology to each other. Similarly, the first variable regions of the HsdS proteins of pLd2, pLd9 and pLd12 are identical, while the second variable region of pLd2 does not show significant homology to those of pLd9 and pLd12. The high similarities of one of the variable regions suggest homologous recombination events as also found for plasmids pAH33 and pAH82 (O'Sullivan et al., 2000). This could result in new R/M specificities.

In addition to the many hsdS genes, on pLd7 also HsdR and HsdM subunits are encoded. These proteins have a low homology to their chromosomal counterparts ( $41 \%$ and $34 \%$, respectively), although the C-terminus of HsdM is quite similar to its chromosomal counterpart. As Schouler et al. (1998) described before, the Cterminal parts of the HsdM subunits of different families have a common sequence that could be involved in the association of HsdM with HsdS. Therefore, the different HsdM and HsdR variants found on the chromosome and the plasmids in combination with the many different plasmid encoded HsdS subunits can form an effective recombination system for broadening the target specificity of the system. 

MAKIDDSVKKKVPELRFPGFTDDWEERKLGSLTTVVRG--ASPRP IQDPKWFDKESDI GWLRIADVTEQNGRIYHLEQHISKLGQEKT MAKIDDSVKKKVPELRFPGFTDDWEERKLGSLTTVVRG--ASPRP IQDPKWFDKESDI GWLRIADVTEQNGRIYHLEQH I SKLGQEKT MAKIDDSVKKKVPELRFPGFTDDWEERKLGSLTTVVRG--ASPRPIQDPKWFDKESDIGWLRIADVTEQNGRIYHLEQHISKLGQEKT MAKIDDSVKKKVPELRFKGFTDDWEERKLGELSNIVGG--GTPSTSNSEYW---DGDIDWYAPAEIGEQR-YVSKSKKTITELGLKKS ------MSKKSPQLRFEGFTDDWELRKLGELLTEVKR----PIKMQDDDT--------YQLVTVKRRN------EGIVSRGFEKG MAKIDDSVKKKVPELRFPGFTDDWEERKLGELTTSFSG--GTPSAGNSSYY---KGDI PFIRSGEINSDK-----TELFLTEAGLKS S MAKIDDSVKKKVPELRFKGFTDDWEERKLGEVGKTKSG-IGFPDAQQG-----GKQVTPFYKVSDMNNPGNEVVMMNANNYASDSQLK

FDRWMGNNRLE--KGDVVFTTEAPL-GNVAQVPDNNG-YILNQRAVAFKSLQE-TDDNFF--AQLLRSPIVQNTLKASSSGGTAKGIG --RVLTEPHL------LLSIAATV-GKPVVNYVKTG---VHDGEL-I FLNPT-EERE FM--EQWLEMFRPKW--QKYGQPGSQVNLN --RVLTEPHL-------LLSIAATV-GKPVVNYVKTG---VHDGFL-IFLNPT-EEREFM--FQWLEMFRPKW--QKYGQPGSQVNLN --RVLTEPHL-------LLSIAATV-GKPVVNYVKTG---VHDGFL-IFLNPT-EERE FM--FOWLEMFRPKW--QKYGQPGSQVNLN SARI LPVGTV-------LFTSRAGI-GNTAILGKEAT---TNQGFQS IVPNPNKLDSYFI--YSRTNELKRYG--EVTGAGSTEVEIS R-QILVKNYFELHAGDYLISKRQVVHGANG IVPKDMEGAIVSNEYLVSVGNKN-ITTDFLTI ISKLPIMYKMFFLSSYGIDIEKLVEN SAKMVSVGDI-------LYALYGATSGEVGISQINGA---INQAI LAIKPCDG-YNSHFL--MQWLKLKKQKI-IDQYLQGG-QGNLS ENKWNPINPQ-------NSGVVFAKVGAAI ELDRKRI---VDTSFLI DNNMMSYLFDSSWNRYFGKTLFEKLRLSIFAQVGALPS ENG

MKEFAKLNARVPETHEEQRKIGLFFKQLDDTIVLHQRKLDLLKEQKKGYLQKMFPKNGSKI PELRFAEFADDWEERKLGEVATFLNGR SELVRNQEI VLP-NYKEQQKIGSFFKQLDETIALHQRKLDLLKEQKKGYLQKMF PQNGAKVPELRFAGFADDWEERKFKD I LKTHSFR SELVRNQEIVLP-NYKEQQKIGSFFKQLDETIALHQRKLDLLKEQKKGYLQKMFPQNGAKVPELRFAGFADDWEERKFKD ILKTHSFR SELVRNQEIVLP-NYKEQQKIGSFFKQLDNTITLHQRKLDLLKEQKKGYLQKMF PKNGAKVPELRFAGFADDWEERKLSSMTNYKNGK GKQMS KMS IMVP-ELSEQKKIGSFFEQLDNTIALHQRKLDLLKEQKKGYLQKMF PKNGAKVPELRFAGFADDWEERKLSSMTNYKNGK VKDWKKREISIP-SLQEQDRISSFFKQLDDTIVLHQRKLDLLKEQKKGYLQKMF PKNGAKVPELRFAGFADDWEERKLSSMTNYKNGK GSIVKNLVLKVP-NEEEQKKIGAFFKQLDDTITLHQRKLDLLKEQKKGYLQKMFPKNGAKVPELRFAGFADDWELRKLGDVVGEIQSG S-DVEDIKVMIP-EESEQKMIGDMFEKLDDIIALHQ RKLDLLKEQKKAFYKRCLYRVYN-

AYKODELLDSGKYKVLRVGNFYTNDSWYYSN--MELGDKYYVDKGDLVYTWS -ATFGPH--IWSGEKVIYHYHIWKVEI SYLAGVS-ENGEYEVIQQGDKPIVG-YSDGEPETDYKDI-TLFGDHTVSLYK-----------PK---- SPFFVATDGVKILSA SYLAGVS-ENGEYEVIQQGDKPIVG-YSDGEPFTDYKDI-TLFGDHTVSLYK----------PK----SPEFVATDGVKILSA SHEDKQS-TSGKLELINLNSISISGGLKHSGKFIDEADD-TLQKDDLVMI LSDVGHGDLLGRVALIPE-----DDRFVLNQRVALLRP SHEDKQS-TSGKLELINLNSIS ISGGLKHSGKF IDEADD-TLQKDDLVMILSDVGHGDLLGRVALIPE-----DDRFVLNQRVALLRP GHEDKQS-T IGKFELINLNSISISGGLKHSGKF IDEADD-TLQKDDLVMILSDVGHGDLLGRVALIPE-----DDRFVLNQRVALLRP NRLPKDSLTNGDVP-------YVVAQTKNDGVETKIARG-TLDYNGKPMKLF---PGNSISFS IDNPEAMFYRNAEFYTSNIMRVIHE

SKFLDRNFTLQLLEADKARLLSSTNGSTMI HVTKGDMESKIVS I PNI DEQKQIGSFFKQLDNTITLHQRKLDLLKEQKKGFLQKMF DNF-DGDYLYTTLE--RYKPEPQGYKRHFT ILKNQDV----WFTENMEEQQKIGSFFKQLDDTIALHQRKLDLLKEQKKGFLQKMFV DNF-DGDYLYTTLE--RYKPEPQGY KRHFT I LKNQDV----WFTENMEEQQKIGSFFKQLDDT IALHQRKLDLLKEQKKGFLQKMFV NTTADPQFLFSY INAHQYYFKAQGAGMSQLN I SKGSVENF I SFVP I IEEQKKIGAFFKQLDDT ITLHQRKLDLLKEQKKGFLQKMFF NTTAD PQFLFSY INAHQYY FKAQGA GMSQLN I SKGSVENF I S FVP I I EEQKKIGAFFKQLDDT ITLHQRKLDLLKEQKKGFLQKMF F NATAD PQFL F SY INAHQYY FKAQGAGMSQLN I SKGSVEN F I S FV I I EEQKKIGTFFKQLDNT ITLHQRKLDLLKEQKKGFLQKMFV DHLTHEQYVFILENMKRF---IRGYDWSR-KFSGPVVSNLEYLQPSEEEQQKIGSFFKQLDDTIDLHQRKLDLLKEQKKGYLQKMFV

Figure 3.4: Alignment of predicted amino acid sequences of HsdS proteins encoded by genes of Lactococcus lactis FM03P. The conserved regions are shown with boldface letters (Schouler et al., 1998). Shaded boxed show the identical amino acids sequence in the variable regions of different HsdS proteins.

In addition to the type I R/M system, plasmid pLd8 harbours orf33, orf34 and orf35 encoding a putative type II restriction-modification system. A similar operon is found in Leuconostoc mesenteroides LK-151 (90\%) and in the strains JM3, SK110 and N41 of Lactococcus lactis. orf34 is predicted to encode a type IIG restriction enzyme/N6-adenine DNA methyltransferase according to the restriction enzymes database REBASE (Roberts et al., 2014), which contains both a methylase and recognition domain (pfam12950) according to the SMART database (Letunic et al., 2014). However, no restriction domain was found in the protein encoded by orf34. The restriction domain is most likely part of the protein encoded by orf33. This protein contains a putative phospholipase D (PLD) domain (pfam13091), a SNF2 ATPase domain (pfam00176), a DEAD-like helicase domain and a helicase C-terminal domain (pfam00271) according to the SMART database. The PLD domain is the metal-independent catalytic site in type IIS restriction 
endonucleases (Pingoud et al., 2014) and might also catalyse the restriction. Notably, the predicted domains in the proteins encoded by orf33 and orf34 are similar to the domains found in the recently identified Class I DISARM system for bacteriophage defence in which the methylase was also predicted to be of the IIG type (Ofir et al., 2017), although they do not share a similar organisation.

Finally, plasmid pLd9 carries an abi gene encoding a putative abortive infection system. The first 642 nucleotides of this gene are $100 \%$ identical to a gene encoding the characterised abortive infection system Abi-859 (Deng et al., 1997). However, the last 207 nucleotides are missing in pLd9 due to an insertion of a mobile element, so this system might not be functional.

\section{Exopolysaccharide (EPS) production}

Exopolysaccharides are thought to have several functions including protection against low-moisture environments and toxic compounds (e.g. bile salts and hydrolysing enzymes, metal ions and antibiotics), colonisation and preventing phage infection (Broadbent et al., 2003). However, in the dairy industry the most important function of EPS is that due to its water-binding capacity, it may improve the rheological properties of the fermented product by affecting the viscosity, syneresis, firmness and taste perception (Zeidan et al., 2017). Plasmid pLd10 carries 18 eps genes encoding putative proteins involved in polysaccharide production via the Wzy-dependent pathway. A typical eps gene cluster in $L$. lactis consists of the 6 highly conserved genes epsR, epsX, epsA, epsB, epsC and epsD at the $5^{\prime}$ end, a variable region including genes encoding a polymerase $(w x y)$, a flippase $(w z x)$, one or more glucosyl tranferases and/or other polymer-modifying enzymes and the conserved genes epsL and orfY at the $3^{\prime}$ end (Zeidan et al., 2017). These genes are usually transcribed as a single mRNA (van Kranenburg and de Vos, 1998). Interestingly, in pLd10 the eps genes are distributed over two clusters and in between these clusters a replication and a mobilisation operon are located, which are flanked by IS elements (Fig. 3.1). The first cluster consists of: i) the epsR gene encoding an XRE family transcriptional regulator; ii) the conserved gene eps $X$ with unknown function; iii) the phosphoregulatory module consisting of epsABC; iv) epsD encoding an undecaprenyl-phosphate galactosephosphotransferase which catalyses the first step in the assembly of the EPS basic repeating unit (i.e. addition of galactose-1-phosphate to the lipidphosphate carrier); v) epsE encoding a putative glycosyl tranferase; and finally (vi) epsL and orfy with an unknown and regulator function, respectively. The second cluster consists of 6 putative glycosyltransferase genes (epsH, epsI, eps], $e p s O, e p s P, e p s Q)$ of which epsI is most likely not functional, 2 putative flippases 
L. lactis FM03P plasmid pLd10
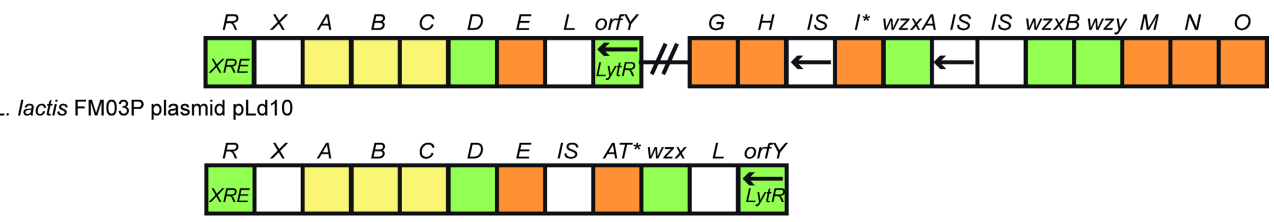

L. lactis FM03P, chromosome operon 1

L. lactis FM03P, chromosome operon 2
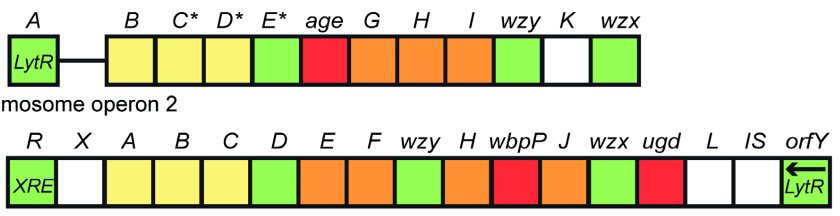

L. lactis KLDS 4.0325

Lactobaciilus delbrueckii Lfi5

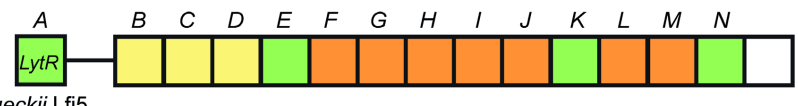

\begin{tabular}{|l|l|l|l|} 
Assembly & Modulation & GT & NDP-sugar biosynthesis \\
\hline
\end{tabular}

Figure 3.5: Schematic genetic organisation of the exopolysaccharide (EPS) gene clusters in L. lactis FM03P. Typical eps gene clusters of L. lactis and Lactobacillus delbrueckii are shown (Zeidan et al., 2017). Colours represents different functional groups. Genes with unknown functions or functions not related to EPS biosynthesis are shown in white. All genes are transcribed in the forward direction except for a few genes oriented in the opposite direction, marked with arrows. Pseudogenes are marked with an asterisk. The 'GT' functional module consists of glycosyl transferases and other enzymes modifying the repeat unit structure, like the acetyl transferase (AT). This figure is adapted from (Zeidan et al., 2017). IS: transposase; NDP-sugar: nucleotide diphopho-sugar.

for export ( $w z x A$ and $w z x B$ ) and a putative polymerase (wzy). On the chromosome, two other eps clusters are found (Fig. 3.5). The first cluster has a typical organisation of $L$. lactis eps operons (epsRXABCD - epsL-orfY), while the second cluster has a typical Lactobacillus eps cluster. This operon starts with epsA encoding a LytR transcriptional regulator followed directly by the phosphoregulatory module (epsBCD) and does not contain epsX, epsL and orf $Y$.

\section{Cation transport}

Cation transporters play an important role in growth and survival of microorganisms i) by protecting against toxic heave metals, like cadmium, ii) by providing essential metals, like magnesium, or iii) by providing metals that increase stress survival, like manganese. Several cation transporters were found on the plasmids of $L$. lactis FM03P.

Plasmid pLd2 carries genes encoding the proteins CadA and CadC, which are $>99.8$ and $100 \%$ similar to CadA and CadC encoded on plasmids pAH82 of L. lactis 
DPC220 and pND302 of L. lactis M71 and on the chromosome of Streptococcus thermophiles 4134, respectively (Liu et al., 1997, O' Sullivan et al., 2001, Schirawski et al., 2002). The cadCA genes have been shown to provide resistance towards cadmium and zinc in both $S$. thermophiles and L. lactis (O' Sullivan et al., 2001, Schirawski et al., 2002). CadC is a transcriptional repressor which binds to its own promoter region (Endo and Silver, 1995). At high cadmium concentrations, CadC is released from its promoter resulting in the production of cadCA transcript and the expression of cadA (Schirawski et al., 2002). CadA is an ATPase of the Ptype catalysing the efflux of cadmium and zinc (Tsai and Linet, 1993). The gene cadD, which is located on the chromosome, also encodes a cadmium transporter with $44 \%$ similarity in amino acid sequence to the plasmid encoded (pRW001) CadD of Staphylococcus aureus (Crupper et al., 1999).

On plasmid pLd7 we found the corA gene encoding a CorA family transporter, which is expected to be the main $\mathrm{Mg}^{2+}$ uptake system in bacteria but might transport other cations like cobalt or nickel as well (Niegowski and Eshaghi, 2007). The CorA protein on pLd7 is $99 \%$ identical to the CorA protein found on plasmid pSK11P of $L$. lactis SK11. On the chromosome three other CorA family transporters are found as well as two other $\mathrm{Mg}^{2+}$ transporting ATPases. The best match of all these proteins only had $27 \%$ homology to CorA of pLd7. Kehres et al. (1998) observed a high degree of diversity in sequences within the CorA family even within one species and argued that members within the CorA family might have functions other than $\mathrm{Mg}^{2+}$ transport. This may also be the case for some of the CorA proteins found in the genome of L. lactis FM03P.

The third cation transporter on plasmids of L. lactis FM03P is the manganese transporter $\mathrm{MntH}$, which belongs to the Nramp family of transporters for divalent metal ions. $\mathrm{Mn}^{2+}$ ion is an important trace metal required for growth and survival of many bacteria (Nierop Groot et al. , 2005). Several species of lactic acid bacteria accumulate $\mathrm{Mn}^{2+}$ to scavenge toxic oxygen species, especially superoxide radicals $\left(\cdot \mathrm{O}_{2}\right)$. This enables the bacteria to survive oxidative stress conditions (Nierop Groot et al., 2005). Therefore, it is expected that the presence and activity of this transporter could enhance growth in environments with a low manganese concentration and increase survival towards oxidative stress. Plasmid pLd7 and pLd9 encode similar MntH proteins which are $99 \%$ homologous and belong to class $\mathrm{C}$ of the MntH proteins (Richer et al., 2003). The chromosome of L. lactis FM03P harbours three operons that encode manganese transporters: i) a $\mathrm{mntH}$ gene of class $C \beta$, which contains a 344 bp deletion compared to the $m n t H C \beta$ gene of $L$. lactis subsp. lactis ATCC 11454 (Richer et al., 2003), ii) a $m n t H$ gene of class B with $99 \%$ identity to $m n t H$ B of L. lactis subsp. lactis ATCC 11454 (Richer et al., 2003), and iii) a mtsBCA operon encoding an $A B C$ transporter for manganese. The 
mntH genes on the plasmids most likely take over the role of the disrupted chromosomal $\mathrm{mntH} C \beta$ gene.

\section{Stress resistance}

Besides the potential oxidative stress resistance provided by $\mathrm{MntH}$, there are universal stress proteins (UspA) encoded by genes on pLd7 and pLd9 and a cold shock protein CspC encoded on pLd2. This CspC protein is identical to the CspC protein of L. lactis MG1363 (Woufers et al., 1998). Furthermore, orf22 carried by pLd8 encodes a putative large-conductance mechanosensitive channel which acts as osmotic release valve in response to a hypoosmotic shock preventing cell lysis (Booth et al., 2007).

\section{Antibiotic resistance}

Plasmid pLd8 carries orf16 encoding a putative aminoglycoside 3-Nacetyltransferase, which catalyses the acetylation of aminoglycoside antibiotics at the 3-amino group and thereby this gene is potentially involved in resistance towards these antibiotics (Wright, 2005). BLAST analysis revealed highly similar genes (>99\% similarity), which are often found on plasmids in L. lactis, for instance in plasmids pJM3B, pSK11L, pC43 pJM2C, pUC06B, pJM4E, pUC109B, p158C, pCIS8, pJM1A and pUC08A. All these plasmid have in common that they are large $(>47 \mathrm{~kb})$ and carry the lacR-lacABCDFEGX operon for lactose uptake and utilisation and the several oligopeptide transporters.

\section{Miscellaneous beneficial functions}

Other functions encoded by genes on the plasmids that might be beneficial for the host are the putative FAD-dependent D-lactate dehydrogenases on pLd7 and pLd9, which are both $99 \%$ similar to the plasmid encoded putative D-lactate dehydrogenase from $L$. lactis SK11. Siezen et al. (2005) suggested that D-lactate dehydrogenase could play a role in D-lactate utilisation in aerobic cultures, which could increase the external $\mathrm{pH}$ and the conversion to acetate leads to ATP production. However, Tanous et al. (2007) did not find D-LDH activity in pGdh442containing strains, which carry a highly similar dld gene, nor were they capable of growing on M17 containing D-lactate as carbon source. Plasmid pLd8 carries orf18 encoding a C4-dicarboxylate $A B C$ transporter, which function is to transport dicarboxylates such as aspartate, malate, fumarate, succinate and oxaloacetate. Finally, plasmid pLd7 and pLd9 both carry a gene encoding a transporter of the major facilitator superfamily. 


\section{New genes}

The plasmids found in L. lactis FM03P carry several genes that are rarely found before in L. lactis, which could indicate horizontal gene transfer events. BLASTP analysis of the amino acid sequence of the predicted protein encoded by orf 2 on plasmid pLd3 gave mainly hits from Lactobacillus reuteri ( $<29 \%$ similar in amino acids) and contained a DUF3552 domain with unknown function. On pLd4 the fmtB gene, encoding a putative peptidoglycan-binding protein, has the highest homology to Streptococcus thermophilus strain B59671, although the homology only covers $60 \%$ of the gene. Both the orf 2 and the fmtB gene encode large proteins (569 and 1217 amino acids, respectively), account for a large fraction of the plasmids ( 40.3 and $30.4 \%$, respectively), and are located on the only two plasmids which do not carry other genes with known dairy functions. The genes orf33 and orf34 on pLd8 were homologous to genes of Leuconostoc mesenteroides LK-151 encoding a putative type IIG restriction-modification system. Many genes in the eps operon on pLd10 (especially all genes from tra to orf46; Table 3.4) were most similar to Lactobacillus species and not found in Lactococcus species. Finally, various small (pseudo)genes were found which had the highest homology to species other than $L$. lactis. These include orf13, orf14, repC and orf16 on pLd2, which are most similar ( $>96 \%$ ) to genes found in Streptococcus, Lactobacillus and Enterococcus and orf2 and orf3 on pLd4, which are similar to genes of Enterococcus faecalis (99 and 97\%, respectively).

\section{Protoplast-induced curing}

To determine the effect of plasmid content on the growth performance, plasmidcured variants were made by protoplast-induced curing. After cells were harvested, the cell wall was degraded with lysozyme. Subsequently, protoplasts were regenerated on plates and the plasmid content of random selected colonies was analysed by PCR targeting genes on each plasmids followed by gel electrophoresis. The obtained variants are shown in Table 3.1.

\section{Plasmid loss in non-dairy environments}

The plasmids carry several genes important for growth in a dairy environment, but in non-dairy environments the plasmids have to be maintained with limited benefits and plasmid-cured variants might arise. Some plasmids were already lost during a single propagation step in either M17 supplemented with glucose or chemically defined medium containing lactose, showing that these plasmids were segregationally unstable in laboratory conditions in the absence of a selection 
pressure (e.g. bacteriophages, lactose, peptides) (Table 3.1). To determine the effect of the plasmid content on the growth of $L$. lactis in a non-dairy environment, the obtained plasmid-cured variants were grown in LM17 and the optical density at $600 \mathrm{~nm}$ was monitored. The growth curves significantly differed between the variants, showing that the plasmid content did affect the growth in M17 (Fig. 3.6A). The highest maximum optical density was found for variant FM03-V1, which only lost plasmid pLd7 (OD 1.1). Variants 48,49 and 50 had the same maximum growth rate (Fig. 3.6B) but reached a slightly lower maximum OD (between 0.91 and 0.96). These three variants all lost plasmids pLd4, pLd5 and pLd6, indicating that one of these plasmids might carry a gene which resulted in the higher OD. The growth curve of the parent strain FM03P was similar to variants 48,59 and 50, but the maximum growth rate could not be estimated using the two-fold dilution method due to flocculation of this variant in M17. The remaining five variants reached a much lower maximum optical density and also grew slower (Fig. 3.6). Both variant 47 and 63 lost plasmid pLd8 carrying the lacRIaCABCDFEGX operon involved in lactose uptake and utilisation. Therefore, the drop in the growth rate in these variants at an optical density of approximately 0.25 could be caused by limited lactose utilisation and suggests that the lacRIaCABCDFEGX operon is functional when present. Variant 3,5 and 51 were all growing much slower throughout the cultivation. Variant 3 and 5 are the only variants missing plasmid pLd10 carrying the eps genes, while variant 51 is the variant with the lowest number of plasmids since it has lost plasmids pLd4, pLd5, pLd6, pLd7, pLd8, pLd9, pLd11 and pLd12.
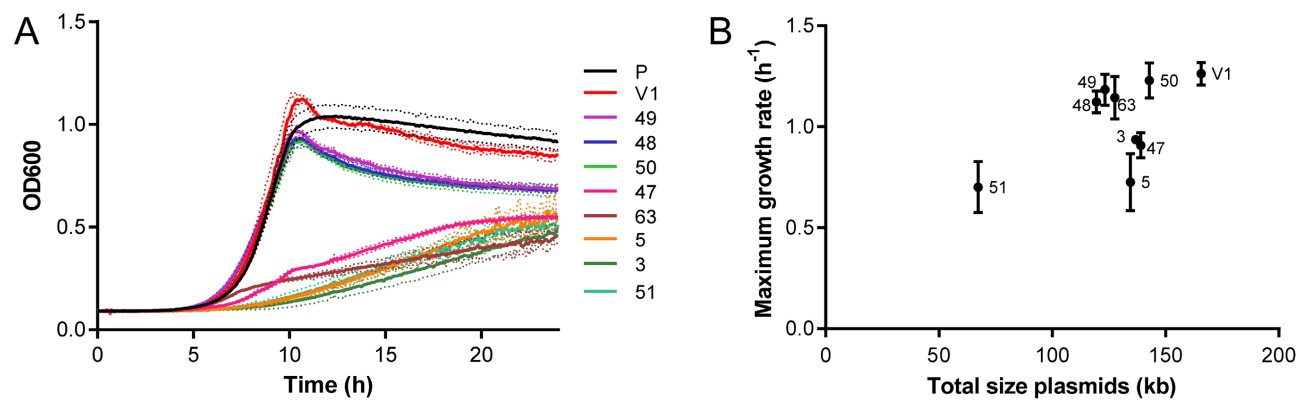

Figure 3.6: Growth of plasmid-cured variants of L. lactis FM03P in LM17. A: Growth curves of the plasmid-cured variants. Dotted lines represent the standard deviation of biological quadruplicates. B: Maximum growth rate as function of the total plasmid size calculated using the two-fold dilution method. Numbers near the symbols correspond to the different variants. The maximum growth rate of the parent strain FM03P could not be estimated using the two-fold dilution method due to flocculation affecting the time to detection and is therefore omitted. Error bars represent the standard deviation of biological triplicates. 
Additionally, growth performance of variants 48 and 50, the latter carrying plasmid pLd2 containing the cadCA operon, was determined in co-cultures in M17 supplemented with $0.5 \%$ glucose and $35 \mu \mathrm{M}$ cadmium. Variant 50 dominated the populations after 27 generations (68\%) which shows that pLd2 provided a competitive advantage in the presence of $35 \mu \mathrm{M}$ cadmium. This is in line with the slightly higher maximal growth rates of variant 50 compared to that of variant 48 in the presence of cadmium (data not shown).

\section{Conclusions}

In the past, important industrial traits have been linked to plasmids in L. lactis. Carrying twelve plasmids, L. lactis FM03P has the biggest plasmidome of all completely sequenced and published $L$. lactis strains up till now. Some of its plasmids were already lost during a single propagation step showing that the plasmids can be easily lost during propagation in a non-dairy environment. Because we directly sequenced the strain after it was isolated from cheese, the risk of plasmid loss was minimised. Examination of the twelve plasmids of L. lactis FM03P showed that 10 of the plasmids carry genes known to be important for the growth and survival in the dairy environment. These genes encode functions such as lactose and citrate utilisation, degradation and uptake of peptides, exopolysaccharide production, cation transport and bacteriophage and stress resistance. This shows that the plasmids play an important role in the adaptation of this strain to the dairy environment. Two plasmids, pLd3 and pLd4, did not carry any genes that are known to be linked to the dairy environment, but both do harbour a large gene with unknown function that has not been found before in L. lactis and may have a function relevant for dairy processing.

\section{Acknowledgements}

We thank Mette Nørtoft Kristensen for isolating the strain. This work was financially supported by Arla Foods, Aarhus, Denmark. 


\section{Supplementary materials}

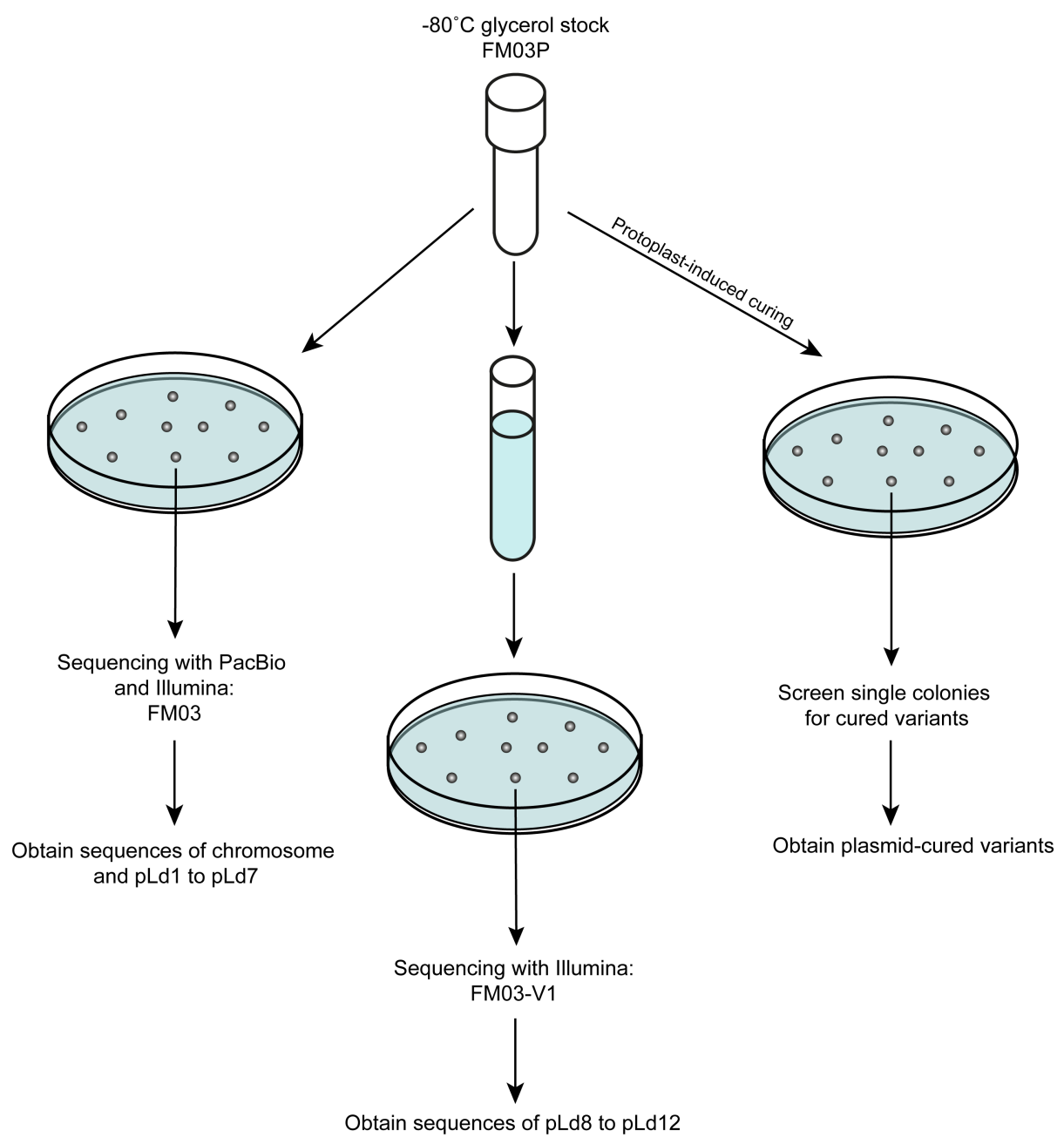

Supplementary Figure S3.1: Summary of the sequencing attempts 


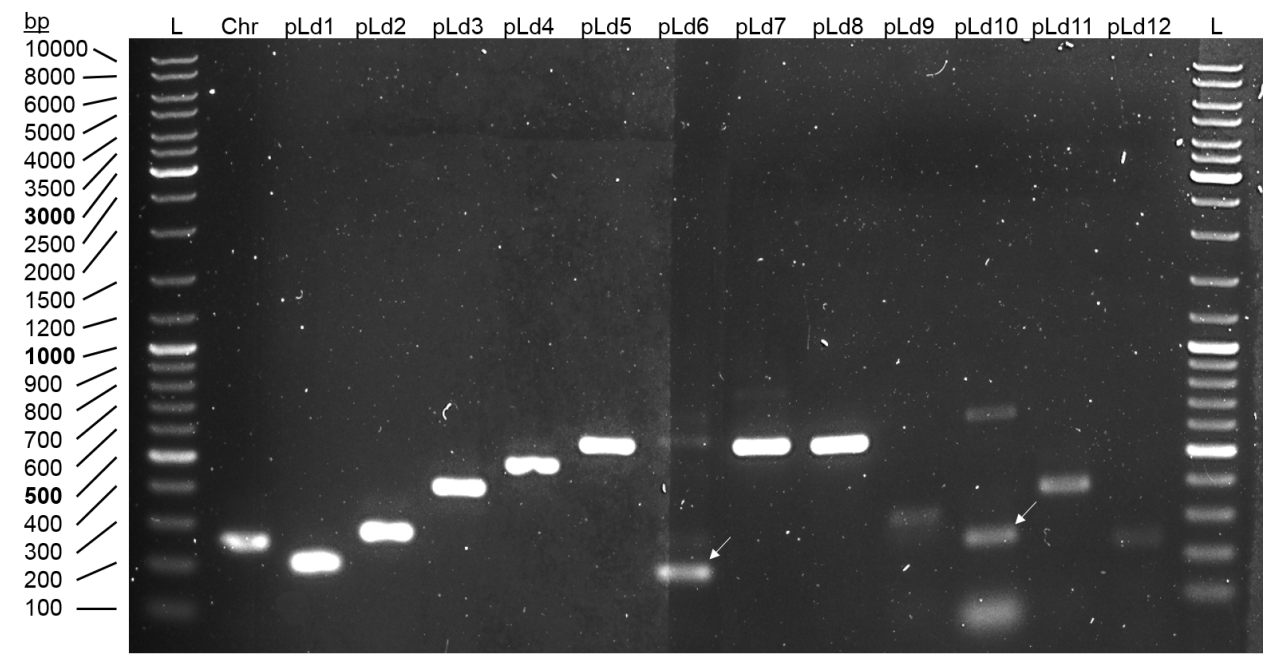

Supplementary Figure S3.2: PCR products confirming the presence of the 12 plasmids in L. lactis FM03P. PCRs were performed using the primers listed in Table 3.2. In case of multiple bands, white arrows indicate the band with the expected size as listed in Table 3.2. L: DNA Ladder mix \#SM1173 (Thermo Sciencific, USA). 
pLd1

pLd4

pLd2

pLd9

pLd7

pLd10 repB2

pLd5

pLd10_repB1

pLd11

pLd8_repB2

pLd 6

pLd12

pLd8_repB1

pLd1

pLd4

pLd2

pLd9

pLd7

pLd10 repB2

pLd5

pLd10_repB1

pLd11

pLd8_repB2

pLd6

pLd12

pLd8_repB1

pLd1

pLd4

pLd2

pLd9

pLd7

pLd10 repB2

pLd5

pLd10_repB1

pLd11

pLd8_repB2

pLd 6

pLd12

pLd8_repB1

pLd1

pLd4

pLd2

pLd9

pLd7

pLd10 repB2

pLd5

pLd10 repB1

pLd 11

pLd8_repB2

pLd6

pLd12

pLd8_repB1

AT-rich

-----TTTTAAATTT----TTGAAAAAAATAAAAAA----------AGGCG-AAGCC--TATTATATATTTATCATATATATTT TTTTGACCCTGATTTTAAGATTGAAAAAAATAAAAAATAGACTTGCCTTAGCA-AGTCC--TTTTATTAATTTATCTTATATATTT -----TTTTAАATTT----TTGAAAAAAATAAAAAA---------TAGGCG-AAGCCTATA-TATATATTTATCTTATATATTT -----TTTTAAATTT----TTGAAAAAAATAAAAAA--------TAGGCG-AAGCCTATA-TATATATTTATCTTATATATTT

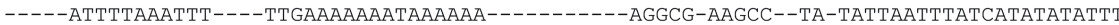
TTTTGATTTTAAATTT----TTGAAAAAAATAAAAAA---------AGGCG-AAGCC--TA-TATTAATTTATCATATATATTT -TTTATTTTTACATTT----TTGAGAAAAATAAAAAA---------TAGGCG-AAGCC--TATTATATATTTATCTTATATATTT -TTTGATTTTAAGATT----TTGAAACGGAGTGAAAA--------AAGGCG-AAGCC--TA-TATATATTTATCATATATATTT -CTTGATTTTAAGATT----TTTAAACGGAGTGAAAA---------AAGGCG-AAGCC--TA-TTATTATTTATCTTATATATTT -----------TT----TTAAAAGGTTATCAAAA---------AAGGCGTTAGCC--TA-TTTATATTTATCATATATATTT TTTTCTTTTTAATTCTTTG-TTGAAAAGTTTTA------------------AAGCC--TA-TATTAATTTATCTTATATATTT TTTTCTTTTTAATTCTTTG-TTGAAAAGTTTTA--_---------------AAGCC--TA-TATTAATTTATATTATATATTT ---------TAT----TTTAAAAAAAGAAAATTGGGACTC-CTTAG----AGTCCCTTTTAATTATTTTATATTATATATTT

AT-rich

$22-\mathrm{bp}$ DR

TAATCTTTTGTTCTTTGCGTGAAAAAAAAGGCAGTGTTTTCGCTAGTTATAGAAATTAAACAGTCACAAAAATCGATGTATAGAG TAATCTTTTGTTCTTTTGTGTGGTTTAAAAGTCAATGTTTTCAAAAATTTACAGATTTATAGCGGTAGAAAAAACTGTGTATAGCG TAATCTTTTATTCTTTTGCGTCAAAAAAAAATCAATATTTTCAAGGCTTTATAGAATTATATACCAACAAAAAACTGTGTATATAC TAATCTTTTATTCTTTTGCGTCAAAAAAAAATCAATATTTTCAAGGCTTTATAGAATTATATACCAACAAAAAACTGTGTATATAC TAATCTTTTCTTCTTTTGCGTCAAAAAAAAGTGAGTGTTTCAAGGGGTTAAAGAATAATATGTGGAGAAAAAACTGTTTATATAT TAATCTTTTCTTCTTTTGCGTCAAAAAAAAGTGAGTATTTTTAAGGGGTTACAGAATAATATAGCATAAAAAAACTGTGTATATAG TAATCTTTTA-TCTTTTGCGTGAAAAAAAAGTCAGTATTTATAGGCTTATACAAATTTTTATAGCAAGAAAAAACTATGTATATAG TAATCTTTTGTTCTTTTGCGTGAGGAAAAAGTCAGTTTTTTCAAGGGGATACAGATTTTTTAGGAGAGAAAAAACTATGTATAAGG TAATCTTTTGTTCTTTTGCGTGAAAAAAAAGTCAGTGTTTGCAAGGTTAAATAGATTTATACCGAGAGAAAAAACAATGTATACCG TAATCTTTTGTTCTTTTACGTGGTGAAAAAGTCAATGTTTCCGCCAATTATATAAATTATACCAAGACAAAAAACAATGTATACCA TAATCTTTTA-TCTTTTGCGTGAAAAAAAAGTCAGTGTTTTCAAGGGTATGCAGAATTTAATACGTAAAAAAAACTGTGTATACAC TAATCTTTTA-TCTTTTGCGTGAAAAAAAAGTCAGTGTTTCCAAGGGTATGCAGAATTTAATACGTAAAAAAAACTATGTACACAC T-GTCTTTTGTTCTTTTGCGAAAAAAAAAATCTAGTGTTTGCAAGGGGTAACAGGATTATAGTCCTACAAAAAACTGTGCATAGTC

$$
22-\mathrm{bP} D R
$$$$
\text { 22-bp DR }
$$

TCACAAAAATCGATGTATAGAGTCACAAAAATCGATGTACACAGTACGA---CTTTTGTATTTGTGTGCTGTATATAGTATAAT GTAGAAAAAACTGTGTATAGCGGTAGAAAAAACTGTGTATAGCGGTAGAAAA-TTATTTTATTATTACCGCTATTGTGATATAAT CAACAAAAAACTGTGCATACACCAACAAAAAACTGTGCATATACCAACTTCT-TTGTTTGTTTCGTTGGTATATAATGATATAAT CAACAAAAAACTGTGCATACACCAACAAAAAACTGTGCATATACCAACTTCT-TTGTTTGTTTCGTTGGTATATAATGATATAAT GGAGAAAAAACTGTTTATATATGGAGAAAAAACTGTTTATACATGGAGAATG-TCTATTATCTTCTCCATGTATTATGGTATAAT САTAАAАAАACTGTGTATATAGCATAAAAAAACTGTGTATATAGCATAAAAA-AATCATCAGTTTATGCTATATTATGATATAAT CAAGAAAAAACTATGTATATAGCAAGAAAAAACTATGTATATAGCAAGAAAA-AACTATAAAAACTTGCTGTATTATGATATAAT AGAGAAAAAACTATGTATAAGGAGAGAAAAAACTATGTATAAGGAGAGAAAA-ATTTATCGTTCCTCTCTTTATTATGATATAAT AGAGAAAAAACAATGTATACCGAGAGAAAAAACAATGTATACCGAGAGAAAATAAATGTTTTTTCTCTCGGTATTTTGATATAAT AGACAAAAAACAATGTATACCAAGACAAAAAACAATGTATACCAAGACAATT-TATTAAAAAATGTCTTGGTAT-GTGATATAAT GTAAAAAAAACTGTGTACACGCGTAAAAAAAACTGTGTACACACGTAAAAAA--ACTTTATTAGTTTACGTGTTTTTGATATAAT GTAAAAAAAACTATGTACACACGTAAAAAAAACTATGTACACACGTAAAAAA---CTTTATTAGTTTACGTGTTTTTGATATAAT CTACAAAAAACTGTGTATAGTCCTACAAAAAACT GTGTATAGCCCTACAAGT-TATTTGTGTTTGTAGGTGTTTCGTGTTATTAT

IRb

(1)

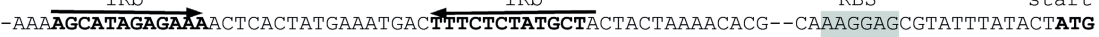
AAAAAGCATAGAGAAATTCACGACTAAATGACTTTCTCTATGCTACTCTCAAA-CACG--CAAAGGAGCGTATTTATATTATG -AAAAGCATGAAGAA-TCTCTCTACGAAAAGTGTTTCTTCATGCTTATCTAAACTCACTCACAAAGGAGCAGTTTT---CTATG -AAAAGCATGAAGAA-TCTCTCTACGAAAAGTGTTTCTTCATGCTTATCTAAACTCACTCACAAAGGAGCAGTTTT---CTATG -AAAAGCATGAAGAA-ACACTTTTCGTCGAGAGAATCTTCATGCGTATCTAAAAACACT--CAAAGGAGCGTATTT---CTATG -AAAAGTATGAAGAACAAACTTTTGAACGAGAATTTCTTCATACTTACTTATGAACACG--CAGAGGAGCGTATCT---TTATG -AAAAGCATAGAGAAAATTCACGACGAAATGA-CTTCTCTATGCTTAGCCAAAATTACTC-ATAAGGAGCAACTTC---TCATG -AAAAGCATGAAGAAATTCCGTCTAAAGAATT-TTTCTTCATGCTTAACCAAAATTACTC-ATAAGGAGCAACTTC---TCATG -AAAAGCATGAAGAAATTCCGTCCAAAGATAT-TTTCTTCATGCTTAACTAAAATTACTC-ATAAGGAGCAACTTC---TCATG -AAAAGCATAGAGAAATCAGACGGAAAAATCAGTTTCTCTATGCTTAACCAAAATTACTC-ATAAGGAGCAACTTC---TCATG -AAAAACATAGAGAAATTGACTCGCTAAAGATTTTTCTCTATGCCTATTTAAAAACACTCACAAAGGAGTA--TTA---CTATG -AAAAACATAGAGAAATTCACTCGGAAAAATGTTTTCTCTATGCCTATCTAAAATCACTCACAAAGGAGTA--TTT---ACATG --

Supplementary Figure S3.3: Multiple sequence alignment of repB promoters of thetatype replication plasmids in L. lactis FM03P. Indicated are the AT-rich regions, the 22-bp direct repeats and in bold the inverted repeats IRa and IRb. The extended -10 promoter site and ribosome binding site (RBS) are shaded. The ATG start codon of repB is shown at the end of the alignment in bold. 
pLd1

pLd2

pLd9

pLd4

pLd7

pLd10 RepB2

pLd6

pLd12

pLd8 RepB2

pLd1 $\overline{0}$ RepB1

pLd11

pLd5

pLd8_RepB1

pLd1

pLd2

pLd9

pLd4

pLd7

pLd10 RepB2

pLd 6

pLd12

pLd8_RepB2

pLd10_RepB1

pLd11

pLd5

pLd8_RepB1

pLd1

pLd2

pLd9

pLd4

pLd7

pLd10 RepB2

pLd6

pLd12

pLd8 RepB2

pLd10_RepB1

pLd11

pLd5

pLd8_RepB1

pLd1

pLd2

pLd9

pLd4

pLd7

pLd10 RepB2

pLd6

pLd12

pLd8 RepB2

pLd10_RepB1

pLd11

pLd5

pLd8_RepB1

pLd1

pLd2

pLd9

pLd4

pLd7

pLd10_RepB2

pLd6

pLd12

pLd8_RepB2

pLd10_RepB1

pLd11

pLd5

pLd8_RepB1
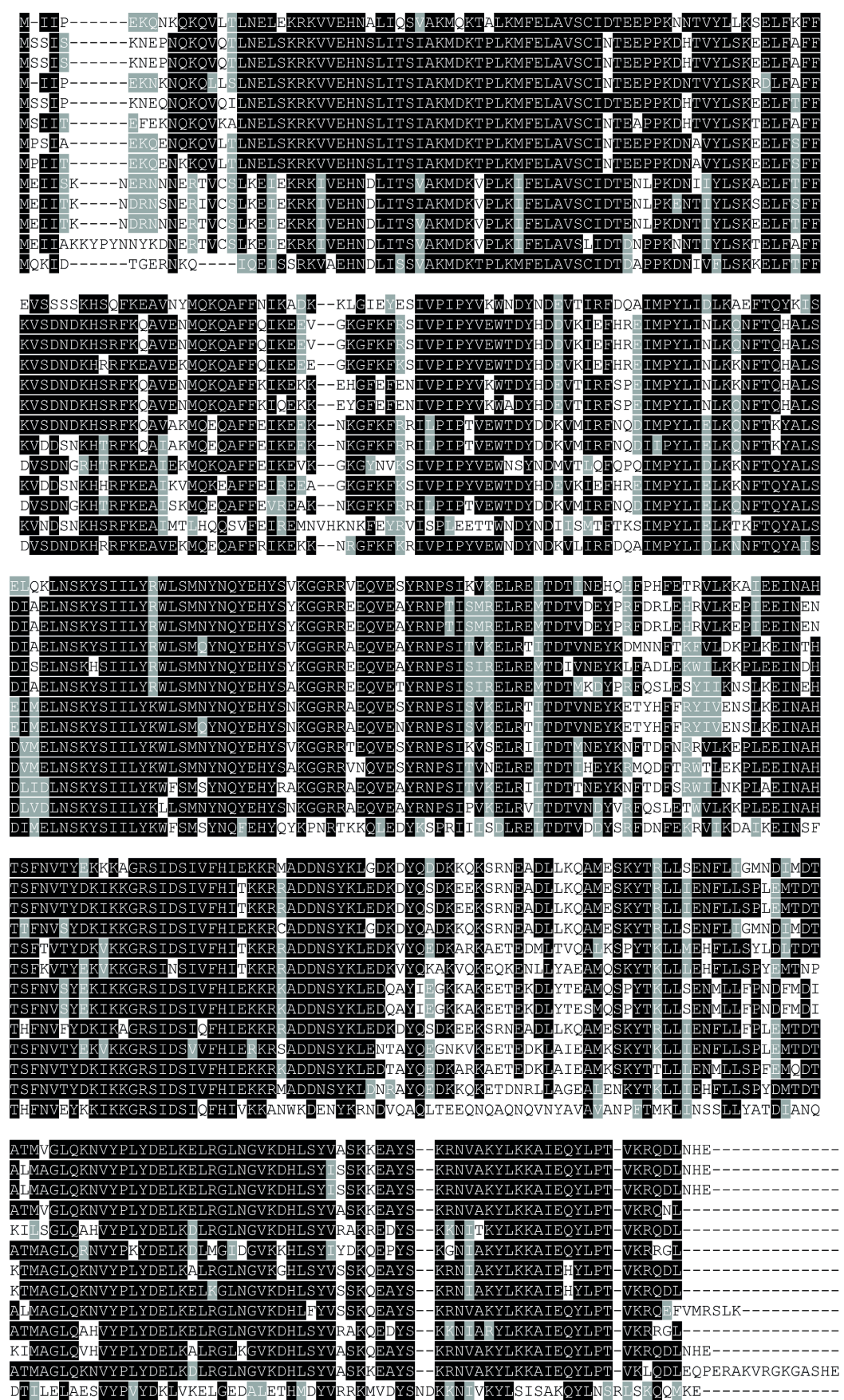

Supplementary Figure S3.4: Multiple alignment of RepB amino acid sequences of thetatype replication plasmids of $L$. lactis FM03P. Amino acids are shaded black if they follow the consensus sequence (same amino acid in at least half of the sequences). Amino acids are shaded grey if they are similar to the consensus. 


\section{References}

Ainsworth, S., Stockdale, S., Bottacini, F., Mahony, J. and van Sinderen, D. (2014). The Lactococcus lactis plasmidome: much learnt, yet still lots to discover. FEMS Microbiol. Rev. 38(5): 1066-1088.

Aleksandrzak-Piekarczyk, T., Kok, J., Renault, P. and Bardowski, J. (2005). Alternative lactose catabolic pathway in Lactococcus lactis IL1403. Appl. Environ. Microbiol. 71(10): 6060-6069.

Aziz, R. K., Bartels, D., Best, A. A., DeJongh, M., Disz, T., Edwards, R. A., Formsma, K., Gerdes, S., Glass, E. M., Kubal, M., Meyer, F., Olsen, G. J., Olson, R., Osterman, A. L., Overbeek, R. A., McNeil, L. K., Paarmann, D., Paczian, T., Parrello, B., Pusch, G. D., Reich, C., Stevens, R., Vassieva, O., Vonstein, V., Wilke, A. and Zagnitko, O. (2008). The RAST Server: rapid annotations using subsystems technology. BMC Genomics 9: 75.

Bachmann, H., Molenaar, D., Kleerebezem, M. and van Hylckama Vlieg, J. E. T. (2011). High local substrate availability stabilizes a cooperative trait. ISME J 5(5): 929-932.

Bandell, M., Lhotte, M. E., Marty-Teysset, C., Veyrat, A., Prévost, H., Dartois, V., Diviès, C., Konings, W. N. and Lolkema, J. S. (1998). Mechanism of the citrate transporters in carbohydrate and citrate cometabolism in Lactococcus and Leuconostoc species. Appl. Environ. Microbiol. 64(5): 1594-1600.

Biesta-Peters, E. G., Reij, M. W., Joosten, H., Gorris, L. G. and Zwietering, M. H. (2010). Comparison of two optical-density-based methods and a plate count method for estimation of growth parameters of Bacillus cereus. Appl. Environ. Microbiol. 76(5): 1399-1405.

Booth, I. R., Edwards, M. D., Black, S., Schumann, U. and Miller, S. (2007). Mechanosensitive channels in bacteria: signs of closure? Nat. Rev. Microbiol. 5(6): 431-440.

Broadbent, J. R., McMahon, D. J., Welker, D. L., Oberg, C. J. and Moineau, S. (2003). Biochemistry, genetics, and applications of exopolysaccharide production in Streptococcus thermophilus: a review. J. Dairy Sci. 86(2): 407-423.

Cavanagh, D., Fitzgerald, G. F. and McAuliffe, O. (2015). From field to fermentation: the origins of Lactococcus lactis and its domestication to the dairy environment. Food Microbiol. 47: 45-61.

Cowan, G. M., Gann, A. A. F. and Murray, N. E. (1989). Conservation of complex DNA recognition domains between families of restriction enzymes. Cell 56(1): 103-109.

Crupper, S. S., Worrell, V., Stewart, G. C. and Iandolo, J. J. (1999). Cloning and expression of cadD, a new cadmium resistance gene of Staphylococcus aureus. J. Bacteriol. 181(13): 4071-4075.

de Felipe, F. L., Magni, C., de Mendoza, D. and López, P. (1995). Citrate utilization gene cluster of the Lactococcus lactis biovar diacetylactis: organization and regulation of expression. Mol. Gen. Genet. 246(5): 590-599.

del Solar, G., Giraldo, R., Ruiz-Echevarría, M. J., Espinosa, M. and Díaz-Orejas, R. (1998). Replication and control of circular bacterial plasmids. Microbiol. Mol. Biol. Rev. 62(2): 434-464.

Deng, Y.-M., Harvey, M. L., Liu, C.-Q. and Dunn, N. W. (1997). A novel plasmid-encoded phage abortive infection system from Lactococcus lactis biovar. diacetylactis. FEMS Microbiol. Lett. 146(1): 149-154.

Drider, D., Bekal, S. and Prevost, H. (2004). Genetic organization and expression of citrate permease in lactic acid bacteria. Genet. Mol. Res. 3(2): 273-281.

Endo, G. and Silver, S. (1995). CadC, the transcriptional regulatory protein of the cadmium resistance system of Staphylococcus aureus plasmid pI258. J. Bacteriol. 177(15): 4437-4441.

Erkus, O., de Jager, V. C. L., Spus, M., van Alen-Boerrigter, I. J., van Rijswijck, I. M. H., Hazelwood, L., Janssen, P. W. M., van Hijum, S. A. F. T., Kleerebezem, M. and Smid, E. J. (2013). Multifactorial diversity sustains microbial community stability. ISME J 7(11): 2126-2136. 
Fallico, V., McAuliffe, O., Fitzgerald, G. F. and Ross, R. P. (2011). Plasmids of raw milk cheese isolate Lactococcus lactis subsp. lactis biovar diacetylactis DPC3901 suggest a plant-based origin for the strain. Appl. Environ. Microbiol. 77(18): 6451-6462.

Francia, M. V., Varsaki, A., Garcillán-Barcia, M. P., Latorre, A., Drainas, C. and de la Cruz, F. (2004). A classification scheme for mobilization regions of bacterial plasmids. FEMS Microbiol. Rev. 28(1): 79-100.

Fujita, Y., Okamoto, T. and Irie, R. (1983). Protoplast rormation and regeneration in lactic streptococci. Agric. Biol. Chem. 47(9): 2103-2105.

García-Quintáns, N., Magni, C., de Mendoza, D. and López, P. (1998). The citrate transport system of Lactococcus lactis subsp. lactis biovar diacetylactis is induced by acid stress. Appl. Environ. Microbiol. 64(3): 850-857.

Kehres, D. G., Lawyer, C. H. and Maguire, M. E. (1998). The CorA magnesium transporter gene family. Microb. Comp. Genomics 3(3): 151-169.

Kelleher, P., Bottacini, F., Mahony, J., Kilcawley, K. N. and van Sinderen, D. (2017). Comparative and functional genomics of the Lactococcus lactis taxon; insights into evolution and niche adaptation. BMC Genomics 18(1): 267.

Kelly, W. J., Ward, L. J. and Leahy, S. C. (2010). Chromosomal diversity in Lactococcus lactis and the origin of dairy starter cultures. Genome Biol. Evol. 2: 729-744.

Khan, S. A. (1997). Rolling-circle replication of bacterial plasmids. Microbiol. Mol. Biol. Rev. 61(4): 442-455.

Klijn, N., Weerkamp, A. H. and de Vos, W. M. (1995). Detection and characterization of lactose-utilizing Lactococcus spp. in natural ecosystems. Appl. Environ. Microbiol. 61(2): 788-792.

Kobayashi, M., Nomura, M., Fujita, Y., Okamoto, T. and Ohmomo, S. (2002). Influence of lactococcal plasmid on the specific growth rate of host cells. Lett. Appl. Microbiol. 35(5): 403-408.

Leroy, F. and De Vuyst, L. (2004). Lactic acid bacteria as functional starter cultures for the food fermentation industry. Trends Food Sci. Technol. 15(2): 67-78.

Letunic, I., Doerks, T. and Bork, P. (2014). SMART: recent updates, new developments and status in 2015. Nucleic Acids Res. 43(D1): D257-D260.

Liu, C.-Q., Khunajakr, N., Chia, L. G., Deng, Y.-M., Charoenchai, P. and Dunn, N. W. (1997). Genetic analysis of regions involved in replication and cadmium resistance of the plasmid pND302 from Lactococcus lactis. Plasmid 38(2): 79-90.

Marty-Teysset, C., Lolkema, J. S., Schmitt, P., Divies, C. and Konings, W. N. (1995). Membrane potential-generating transport of citrate and malate catalyzed by Citp of Leuconostoc mesenteroides. J. Biol. Chem. 270(43): 25370-25376.

McKay, L. L. and Baldwin, K. A. (1975). Plasmid distribution and evidence for a proteinase plasmid in Streptococcus lactis C2. Appl. Microbiol. 29(4): 546-548.

Mills, S., McAuliffe, O. E., Coffey, A., Fitzgerald, G. F. and Ross, R. P. (2006). Plasmids of lactococci-genetic accessories or genetic necessities? FEMS Microbiol. Rev. 30(2): 243-273.

Murray, N. E. (2000). Type I restriction systems: sophisticated molecular machines (a legacy of Bertani and Weigle). Microbiol. Mol. Biol. Rev. 64(2): 412-434.

Nickels, C. and Leesment, H. (1964). Method for the differentiation and qualitative determination of starter bacteria. Milchwissenschaft 19: 374-378.

Niegowski, D. and Eshaghi, S. (2007). The CorA family: Structure and function revisited. Cell. Mol. Life Sci. 64(19): 2564.

Nierop Groot, M. N., Klaassens, E., de Vos, W. M., Delcour, J., Hols, P. and Kleerebezem, M. (2005). Genome-based in silico detection of putative manganese transport systems in Lactobacillus plantarum and their genetic analysis. Microbiology 151(4): 12291238.

Nomura, M., Kobayashi, M., Narita, T., Kimoto-Nira, H. and Okamoto, T. (2006). Phenotypic and molecular characterization of Lactococcus lactis from milk and plants. J. Appl. Microbiol. 101(2): 396-405.

Novick, R. P. (1987). Plasmid incompatibility. Microbiol. Rev. 51(4): 381.

O' Sullivan, D., Ross, R. P., Twomey, D. P., Fitzgerald, G. F., Hill, C. and Coffey, A. (2001). Naturally occurring lactococcal plasmid pAH90 links bacteriophage resistance and 
mobility functions to a food-grade selectable marker. Appl. Environ. Microbiol. 67(2): 929-937.

O'Sullivan, D., Twomey, D. P., Coffey, A., Hill, C., Fitzgerald, G. F. and Ross, R. P. (2000). Novel type I restriction specificities through domain shuffling of HsdS subunits in Lactococcus lactis. Mol. Microbiol. 36(4): 866-875.

Ofir, G., Melamed, S., Sberro, H., Mukamel, Z., Silverman, S., Yaakov, G., Doron, S. and Sorek, R. (2017). DISARM is a widespread bacterial defence system with broad anti-phage activities. Nature Microbiology: 1.

Passerini, D., Beltramo, C., Coddeville, M., Quentin, Y., Ritzenthaler, P., Daveran-Mingot, M.-L. and Le Bourgeois, P. (2010). Genes but not genomes reveal bacterial domestication of Lactococcus lactis. PLoS One 5(12): e15306.

Perwez, T. and Meyer, R. J. (1999). Stabilization of the relaxosome and stimulation of conjugal transfer are genetically distinct functions of the R1162 protein MobB. $J$. Bacteriol. 181(7): 2124-2131.

Pingoud, A., Wilson, G. G. and Wende, W. (2014). Type II restriction endonucleases-a historical perspective and more. Nucleic Acids Res. 42(12): 7489-7527.

Richer, E., Courville, P., Bergevin, I. and Cellier, M. F. M. (2003). Horizontal gene transfer of "prototype" Nramp in bacteria. J. Mol. Evol. 57(4): 363-376.

Roberts, R. J., Vincze, T., Posfai, J. and Macelis, D. (2014). REBASE-a database for DNA restriction and modification: enzymes, genes and genomes. Nucleic Acids Res. 43(D1): D298-D299.

Salama, M. S., Musafija-Jeknic, T., Sandine, W. E. and Giovannoni, S. J. (1995). An ecological study of lactic acid bacteria: isolation of new strains of Lactococcus including Lactococcus lactis subspecies cremoris. J. Dairy Sci. 78(5): 1004-1017.

Sandine, W., Radich, P. and Elliker, P. (1972). Ecology of the lactic streptococci. A review. J. Milk Food Technol. 35(3): 176-185.

Schirawski, J., Hagens, W., Fitzgerald, G. F. and van Sinderen, D. (2002). Molecular characterization of cadmium resistance in Streptococcus thermophilus strain 4134: an example of lateral gene transfer. Appl. Environ. Microbiol. 68(11): 5508-5516.

Schouler, C., Gautier, M., Ehrlich, S. D. and Chopin, M.-C. (1998). Combinational variation of restriction modification specificities in Lactococcus lactis. Mol. Microbiol. 28(1): 169-178.

Seegers, J. F., Bron, S., Franke, C. M., Venema, G. and Kiewiet, R. (1994). The majority of lactococcal plasmids carry a highly related replicon. Microbiology 140(6): 12911300.

Siezen, R. J., Renckens, B., van Swam, I., Peters, S., van Kranenburg, R., Kleerebezem, M. and de Vos, W. M. (2005). Complete sequences of four plasmids of Lactococcus lactis subsp. cremoris SK11 reveal extensive adaptation to the dairy environment. Appl. Environ. Microbiol. 71(12): 8371-8382.

Siezen, R. J., Starrenburg, M. J., Boekhorst, J., Renckens, B., Molenaar, D. and van Hylckama Vlieg, J. E. (2008). Genome-scale genotype-phenotype matching of two Lactococcus lactis isolates from plants identifies mechanisms of adaptation to the plant niche. Appl. Environ. Microbiol. 74(2): 424-436.

Smid, E. J., Poolman, B. and Konings, W. N. (1991). Casein utilization by lactococci. Appl. Environ. Microbiol. 57(9): 2447-2452.

Smith, M. R., Hugenholtz, J., Mikóczi, P., Deree, E., Bunch, A. W. and Debont, J. A. M. (1992). The stability of the lactose and citrate plasmids in Lactococcus lactis subsp lactis biovar diacetylactis. FEMS Microbiol. Lett. 96(1): 7-11.

Tanous, C., Chambellon, E. and Yvon, M. (2007). Sequence analysis of the mobilizable lactococcal plasmid pGdh442 encoding glutamate dehydrogenase activity. Microbiology 153(5): 1664-1675.

Terzaghi, B. E. and Sandine, W. E. (1975). Improved medium for lactic streptococci and their bacteriophages. Appl. Microbiol. 29(6): 807-813.

Tsai, K. J. and Linet, A. L. (1993). Formation of a phosphorylated enzyme intermediate by the cadA $\mathrm{Cd}^{2+}$-ATPase. Arch. Biochem. Biophys. 305(2): 267-270.

Tynkkynen, S., Buist, G., Kunji, E., Kok, J., Poolman, B., Venema, G. and Haandrikman, A. (1993). Genetic and biochemical characterization of the oligopeptide transport system of Lactococcus lactis. J. Bacteriol. 175(23): 7523-7532. 
van Kranenburg, R. and de Vos, W. M. (1998). Characterization of multiple regions involved in replication and mobilization of plasmid pNZ4000 coding for exopolysaccharide production in Lactococcus lactis. J. Bacteriol. 180(20): 5285-5290.

van Mastrigt, O., Abee, T. and Smid, E. J. (2017). Complete genome sequences of Lactococcus lactis subsp. lactis bv. diacetylactis FM03 and Leuconostoc mesenteroides FM06 isolated from cheese. Genome Announc. 5(28): e00633-17.

van Mastrigt, O., Mager, E. E., Jamin, C., Abee, T. and Smid, E. J. (2018). Citrate, low pH and amino acid limitation induce citrate utilisation in Lactococcus lactis biovar diacetylactis. Microb. Biotechnol. 11(2): 369-380.

van Rooijen, R. J. and de Vos, W. M. (1990). Molecular cloning, transcriptional analysis, and nucleotide sequence of $l a c R$, a gene encoding the repressor of the lactose phosphotransferase system of Lactococcus lactis. J. Biol. Chem. 265(30): 1849918503.

van Rooijen, R. J., Gasson, M. J. and De Vos, W. M. (1992). Characterization of the Lactococcus lactis lactose operon promoter: contribution of flanking sequences and LacR repressor to promoter activity. J. Bacteriol. 174(7): 2273-2280.

Woufers, J. A., Sanders, J.-W., Kok, J., de Vos, W. M., Kuipers, O. P. and Abee, T. (1998). Clustered organization and transcriptional analysis of a family of five csp genes of Lactococcus lactis MG1363. Microbiology 144(10): 2885-2893.

Wright, G. D. (2005). Bacterial resistance to antibiotics: Enzymatic degradation and modification. Adv. Drug Deliv. Rev. 57(10): 1451-1470.

Zeidan, A. A., Poulsen, V. K., Janzen, T., Buldo, P., Derkx, P. M., Øregaard, G. and Neves, A. R. (2017). Polysaccharide production by lactic acid bacteria: from genes to industrial applications. FEMS Microbiol. Rev. 41(Supp_1): S168-S200.

Zhang, S. and Meyer, R. (1997). The relaxosome protein MobC promotes conjugal plasmid mobilization by extending DNA strand separation to the nick site at the origin of transfer. Mol. Microbiol. 25(3): 509-516. 


\section{Chapter 4}

\section{Citrate, low pH and amino acid limitation induce citrate utilisation in Lactococcus lactis biovar diacetylactis}

Oscar van Mastrigt, Emma E. Mager, Casper Jamin, Tjakko Abee \& Eddy J. Smid

Published in Microbial Biotechnology, March 2018, 11(2):369-380 Published online: 7 Dec 2017, doi: 10.1111/1751-7915.13031 


\section{Abstract}

In Lactococcus lactis subsp. lactis biovar diacetylactis citrate transport is facilitated by the plasmid-encoded citrate permease (CitP). In this work, we analysed regulation of citrate utilisation by $\mathrm{pH}$, nutrient limitation and the presence of citrate at four different levels: i) plasmid copy number, ii) citP transcription, iii) citP mRNA processing and iv) citrate utilisation capacity. Citrate was supplied as co-substrate together with lactose. The citP gene is known to be induced in cells grown at low $\mathrm{pH}$. However, we demonstrated that transcription of citP was even higher in the presence of citrate (3.8-fold compared to 2.0-fold). The effect of citrate has been overlooked by other researchers because they determined the effect of citrate using M17 medium, which already contains 0.80 $\pm 0.07 \mathrm{mM}$ citrate. The plasmid copy number increased in cells grown under amino acid limitation (1.6-fold) and/or at low pH (1.4-fold). No significant differences in citP mRNA processing were found. Citrate utilisation rates increased from approximately 1 to $65 \mu \mathrm{mol} . \mathrm{min}^{-1} . \mathrm{gDW}^{-1}$ from lowest to highest citP expression. Acetoin formation increased during growth in an acidic environment due to induction of the acetoin pathway. Quantification of the relative contributions allowed us to construct a model for regulation of citrate utilisation in L. lactis biovar diacetylactis. This knowledge will help to select conditions to improve flavour formation from citrate. 


\section{Introduction}

Citrate is an important precursor for flavour formation in dairy fermentations. In contrast to lactose which can be consumed by many lactic acid bacteria, citrate can be degraded only by particular lactic acid bacteria, such as Lactococcus lactis biovar diacetylactis. These bacteria convert citrate via oxaloacetate into pyruvate (Harvey and Collins, 1961), which can be further converted into acetoin and diacetyl. The latter two compounds are responsible for the buttery flavour of dairy products. The intermediate pyruvate is a central metabolite in the metabolism of lactic acid bacteria and is also the end-product of glycolysis. However, production of acetoin and diacetyl is mainly linked to citrate metabolism because no reducing equivalents are produced during citrate degradation (Collins, 1972, Hugenholtz, 1993).

L. lactis biovar diacetylactis is one of the main species in dairy starter cultures that degrades citrate. In this bacterium the rate of citrate utilisation is limited by the rate of citrate transport across the cell membrane (Harvey and Collins, 1962), which is facilitated by a citrate permease. This permease transports divalent citrate either in symport with a proton or in exchange for monovalent L-lactate (Bandell et al., 1998, Cachon et al., 1995, Marty-Teysset et al., 1995, MartyTeysset et al., 1996). Divalent citrate is most abundant around pH 5.5, which agrees with the observed optimum pH for citrate utilisation (Smith et al., 1992).

The gene encoding the citrate permease (citP) is located on a plasmid in L. lactis biovar diacetylactis (Kempler and Mckay, 1979a, b, 1981). In contrast, all other lactococcal genes required for citrate degradation are located on the chromosome (Martín et al., 2004). The plasmid linked gene citP is part of the citQRP operon (Magni et al., 1994), in which citR codes for a regulatory protein and citQ codes for a leader peptide (de Felipe et al., 1995, Drider et al., 2004). The citQRP operon contains promoter $\mathrm{P} 1$ and $\mathrm{P} 2$, which are located before and after an IS982 element, respectively (de Felipe et al., 1995). Transcription is mainly driven from promoter $\mathrm{P} 1$ resulting in a 2.9-kb mRNA, which is post-transcriptionally regulated by processing in a complex secondary structure (de Felipe et al., 1995, 1996). Garcia-Quintáns et al. (1998) showed that promoter P1 is strongly induced by low $\mathrm{pH}$, which conceivably results in higher citrate uptake rates at low $\mathrm{pH}$.

The effect of citrate on the expression of citP is still under debate (de Felipe et al., 1996). Harvey and Collins (1962) reported a 20 times higher citrate uptake rate of cells grown in batch cultivation on a medium supplemented with citrate compared to growth on a medium without citrate. On the other hand, Smith et al. (1992) did not find any significant difference in citrate uptake in a pulse experiment in chemostat cultivations on LM17 without and with supplemented 
citrate. Expression of citP and the uptake of citrate were also not affected when L. lactis was grown in batch cultivation on LM17 with or without supplementation of citrate (Magni et al., 1994).

The expression of the chromosomal citM-citCDEFXG operon, containing genes encoding the citrate lyase complex and an oxaloacetate decarboxylase, as well as the citrate lyase and oxaloacetate decarboxylase activity increases at low $\mathrm{pH}$ (Martín et al., 2004, Sender et al., 2004). However, citrate does not affect the expression of the citM-citCDEFXG operon and the citrate lyase activity in L. lactis (Cogan, 1981, Martín et al., 2004).

The aim of this study was to decipher the role of different parameters acting on overall citrate utilisation capacity (i.e. uptake and metabolism) in L. lactis biovar diacetylactis, including copy numbers of the citP containing plasmid, citP mRNA levels, citP mRNA processing, uptake and metabolism of citrate, and production of end products, such as acetoin. Bacteria were grown at constant growth rates using chemostat cultivation on chemically defined lactose-containing media at $\mathrm{pH}$ 5.5 and $\mathrm{pH} 7.0$, with and without supplementation of citrate under lactose and amino acid limitation.

\section{Materials and methods}

\section{Strain}

In this study we used Lactococcus lactis subsp. lactis biovar diacetylactis FM03-V1. This variant has the same parent as L. lactis FM03, of which the genome has been sequenced (van Mastrigt et al., 2017). The most important difference between strain FM03-V1, strain FM03 and their parent strain FM03P is the plasmid content: FM03P contains 12 plasmids ( $p L d 1$ till pLd12); FM03 contains 7 plasmids ( $p L d 1$ till pLd7); FM03-V1 contains 11 plasmids (pLd1, pLd2, pLd3, pLd4, pLd5, pLd6, pLd8, pLd9, pLd10, pLd11 and pLd12). Compared to L. lactis FM03, FM03-V1 shows improved lactose utilisation in chemically defined medium. This is most likely caused by the presence of pLd8, which carries genes encoding a phosphotransferase system (PTS) for lactose uptake and the tagatose-6phosphate pathway for lactose utilisation. Both variants contain plasmid pLd1 that encodes the citQRP operon.

\section{Chemostat cultivation}

Chemostat cultivations were carried out in duplicate in bioreactors with a working volume of $0.5 \mathrm{~L}$ (Multifors, Infors $\mathrm{HT}$, Switzerland). The temperature was maintained at $30^{\circ} \mathrm{C}$, a stirring speed of $300 \mathrm{rpm}$ was used and the $\mathrm{pH}$ was 
controlled at 5.5 or 7 by automatic addition of $5 \mathrm{M} \mathrm{NaOH}$. To maintain anaerobic conditions, we flushed the headspace with nitrogen gas at a rate of $0.1 \mathrm{~L} / \mathrm{min}$. Bioreactors were inoculated (inoculum size: $2 \% \mathrm{v} / \mathrm{v}$ ) with an overnight culture in M17 (Terzaghi and Sandine, 1975$)$ supplemented with $0.5 \%$ lactose $(w / v)$. At the end of the exponential growth phase, continuous fresh medium supply was activated to culture the bacteria at a constant growth rate of $0.135 \mathrm{~h}^{-1}$ (chemostat mode). Samples were taken after reaching steady-state conditions. A steady state was considered to be achieved after a minimum of five volume changes at which the optical density at $600 \mathrm{~nm}$ remained constant. The optical density was continuously monitored using an internal probe (Trucell 2, Finesse, USA). The same culture was used to analyse different conditions by changing the medium composition or the $\mathrm{pH}$. Lactose and amino acid-limited cultures were analysed in separate cultivations. Duplicate experiments of the same condition were never performed in the same cultivation.

\section{Media}

The chemically defined media that were used differed in lactose and amino acid content to vary the type of nutrient limitation. Lactose and amino acid-limited cultures contained per $\mathrm{kg}$ of medium 5.3 or $21 \mathrm{~g}$ lactose. $\mathrm{H}_{2} \mathrm{O}(0.5$ or $2 \%)$ and 10 or $1 \mathrm{~g}$ Bacto-Tryptone, respectively. Citrate content was either 2.43 or $0 \mathrm{~g} . \mathrm{kg}^{-1}$ $\left(\mathrm{NH}_{4}\right)_{3}$ citrate. Furthermore, all media contained per $\mathrm{kg}: \mathrm{KH}_{2} \mathrm{PO}_{4}, 2.67 \mathrm{~g}$; Na.acetate. $3 \mathrm{H}_{2} \mathrm{O}, 1.66 \mathrm{~g} ; 100 \mathrm{x}$ metal stock, $10 \mathrm{~g} ; 100 \mathrm{x}$ nucleotide stock, $10 \mathrm{~g}$ and $100 \mathrm{x}$ vitamin stock, $10 \mathrm{~g}$. All media were prepared in $5 \mathrm{~L}$ batches, adjusted the $\mathrm{pH}$ to 5.5 and filter-sterilised (Millipore, USA).

The 100x vitamin stock solution contained per $\mathrm{kg}: 0.2 \mathrm{~g}$ pyridoxine- $\mathrm{HCl}, 0.5 \mathrm{~g}$ pyridoxamine- $\mathrm{HCl}, 0.1 \mathrm{~g}$ nicotinic acid, $0.1 \mathrm{~g}$ thiamin- $\mathrm{HCl}, 0.1 \mathrm{~g} \mathrm{Ca}-(\mathrm{D}+)-$ panthothenate, $1 \mathrm{~g}$ Na-p-aminobenzoate, $0.25 \mathrm{~g}$ D-biotin, $0.1 \mathrm{~g}$ folic acid, $0.1 \mathrm{~g}$ vitamin $B_{12}, 0.5 \mathrm{~g}$ orotic acid, $0.5 \mathrm{~g}$ thymidine, $0.5 \mathrm{~g}$ inosine and $0.25 \mathrm{~g} \mathrm{DL}-6,8-$ thioctic acid. $\mathrm{pH}$ was set at 6.8 . The $100 \mathrm{x}$ nucleic acid stock solution contained per $\mathrm{kg}$ : $1 \mathrm{~g}$ adenine, $1 \mathrm{~g}$ uracil, $1 \mathrm{~g}$ xanthine and $1 \mathrm{~g}$ guanine. The compounds were dissolved in $0.1 \mathrm{M} \mathrm{NaOH}$. The $100 \mathrm{x}$ metal stock solution contained per $\mathrm{kg}$ : $20 \mathrm{~g} \mathrm{MgCl}_{2} .6 \mathrm{H}_{2} \mathrm{O}, 5 \mathrm{~g} \mathrm{CaCl}_{2} .2 \mathrm{H}_{2} \mathrm{O}, 0.5 \mathrm{~g} \mathrm{ZnSO}_{4} .7 \mathrm{H}_{2} \mathrm{O}, 0.25 \mathrm{~g} \mathrm{CoCl}_{2} .6 \mathrm{H}_{2} \mathrm{O}, 1.6 \mathrm{~g}$

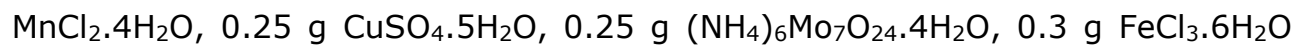
and $0.5 \mathrm{~g} \mathrm{FeSO}_{4} .7 \mathrm{H}_{2} \mathrm{O}$. $\mathrm{FeSO}_{4} .7 \mathrm{H}_{2} \mathrm{O}$ was first dissolved in $10 \mathrm{ml} 17 \% \mathrm{HCl}$, before it was mixed with the other compounds. All stocks were kept at $-20^{\circ} \mathrm{C}$ until use. 


\section{Analysis of extracellular metabolites}

After taking a sample from the bioreactor, cells were directly removed by centrifugation $\left(17000 \times g\right.$ for 2 minutes at $4^{\circ} \mathrm{C}$ ) and the supernatant was stored at $-20^{\circ} \mathrm{C}$ until analysis. High Performance Liquid Chromatography (HPLC) was performed to quantify lactose, lactate, acetate, ethanol, formate, pyruvate and acetoin on an Ultimate 3000 (Dionex) equipped with an Aminex HPX-87H column $(300 \times 7.8 \mathrm{~mm})$ with pre-column (Bio-Rad, USA). As mobile phase, $5 \mathrm{mM}$ sulfuric acid was used at $0.6 \mathrm{ml} / \mathrm{min}$ and the column was kept at $40^{\circ} \mathrm{C}$. Compounds were detected by a refractive index detector Shodex RI-101 (Showa DenkoKK, Japan) for quantification and UV measurements at 220, 250 and $280 \mathrm{~nm}$ for identification.

The citrate concentration was determined with a citric acid kit (Roche, Switzerland) according to the manufacturer's procedures. The decrease of absorbance at $340 \mathrm{~nm}$ was measured, which correlates with the amount of NADH consumed in enzymatic reactions with citrate as substrate. This method was also used for the quantification of citrate in M17.

Extracellular metabolite concentrations were analysed in duplicate.

\section{Cell dry weight determination}

Samples were directly taken from the bioreactor and passed through pre-weighted membrane filters with a pore size of $0.2 \mu \mathrm{m}$ (Pall Corporation, USA) by a vacuum filtration unit. The exact sample sizes were determined by weighing. The membrane filters were washed with at least three volumes demineralised water, dried at $80^{\circ} \mathrm{C}$ for $48 \mathrm{~h}$ and weighted again on an analytical balance. Dry weights were determined in duplicate.

\section{Citrate utilisation capacity}

Citrate utilisation capacity was determined in triplicate by monitoring the decrease in citrate concentration in time. Ten $\mathrm{ml}$ of cell suspension was taken from the bioreactor and directly centrifuged at $5000 \times \mathrm{g}$ for 5 minutes. The pellet was washed with physiological salt solution at $\mathrm{pH} 5.5$, resuspended in modified chemically defined medium of $\mathrm{pH} 5.5$ and incubated at $30^{\circ} \mathrm{C}$. This medium was equal to the lactose-limited medium but did not contain any lactose or acetate. Samples were taken from these cultures at regular intervals followed by direct centrifugation $\left(17000 \times \mathrm{g}\right.$ for $1 \mathrm{~min}$ at $4^{\circ} \mathrm{C}$ ). The supernatant was stored at $-20^{\circ} \mathrm{C}$ until the citrate concentration was determined. In contrast to the enzymatic determination of residual citrate in chemostats, we monitored the citrate concentration using HPLC according to the protocol described before. The citrate 
utilisation rate was calculated by dividing the change in citrate concentration in time, determined by linear regression, by the biomass concentration.

\section{RNA isolation}

Gene expression analysis consisted of four different steps: i) stabilisation of RNA by quenching of cells, ii) isolation of RNA, iii) reverse transcription of RNA into CDNA, and iv) relative quantification of CDNA by qPCR. A two $\mathrm{ml}$ cell suspension sample derived from the bioreactor was directly mixed with $4 \mathrm{ml}$ RNAprotect (Qiagen, Germany) for immediate RNA stabilisation. After mixing to homogeneity the sample was flash-frozen in liquid nitrogen and stored at $-80^{\circ} \mathrm{C}$ until further use. RNA was isolated using the RNeasy Mini kit (Qiagen, Germany). Samples were thawed on ice and $1 \mathrm{ml}$ sample was taken and centrifuged at $17000 \times g$ for 5 minutes at room temperature. The pellet was resuspended in $350 \mu \mathrm{l}$ RLT buffer containing $10 \mu \mathrm{l} / \mathrm{ml} \beta$-mercaptoethanol. The suspension was transferred to a lysis matrix tube (matrix B. $0.1 \mathrm{~mm}$ silica spheres, Bio-Rad) and bead-beated in three cycles of 30 seconds beating (4.0 m.s ${ }^{-1}$; Bio-Rad Fastprep-24) and 5 minutes cooling on ice. Subsequently, tubes were centrifuged for 20 seconds $(17000 \times g)$ and the supernatant was transferred to a new tube. To the supernatant an equal volume of $70 \%$ ethanol was added, thoroughly mixed and transferred to a spin column. The different binding and washing steps were performed according to the manufacturer's procedure. To elute RNA from the column, $30 \mu \mathrm{l}$ RNase-free water was added and the column was centrifuged for 1 minute at $17000 \times g$. This step was repeated once. A small aliquot of the isolated RNA was used for determination of the RNA content using absorbance spectroscopy (Nanodrop; Thermo Scientific, USA). The remaining sample was stored at $-80^{\circ} \mathrm{C}$ until further use.

\section{Reverse transcriptase}

RNA was reverse transcribed using the QuantiTect Reverse Transcription Kit (Qiagen, Germany) according to the manufacturer's procedure. This procedure also removed remaining genomic DNA from the purified RNA samples. RNA used for the reverse transcriptase reaction was standardised at $175 \mathrm{ng}$. After the treatment, the $\mathrm{CDNA}$ was stored at $-20^{\circ} \mathrm{C}$ until analysis of the expression by qPCR.

\section{Relative expression determined by qPCR}

Relative gene expression of the citrate permease (citP) was quantified by qPCR (Bio-Rad Thermal cycler CFX96 Real Time system) using recA as reference gene. recA showed the most stable expression in a set of five housekeeping genes (recA, tuf, gyrB, gyrA and rpoD) during chemostat cultivation in all tested conditions 
(data not shown). qPCR data were analysed with Bio-Rad CFXmanager software. A threshold of 800 Relative Fluorescence Unit (RFU) was used for all measurements. The qPCR reaction took place in $20 \mu \mathrm{l}$ and contained per reaction $10 \mu \mathrm{l} 2 \mathrm{x}$ iQ SYBR green supermix (Bio-Rad, USA), $0.5 \mu \mathrm{M}$ forward and reverse primer and $1 \mu \mathrm{l}$ cDNA. Primer sequences are given in Table 4.1.

Table 4.1: Primer and Taqman probes used for determination of plasmid copy number, relative expression of citP and processing of citP mRNA. The gene glyA, located with one copy on the chromosome, was targeted in the plasmid copy number determination. recA was used as reference gene in the relative citP expression assays. Primers for citP were used for determination of both plasmid copy number, relative citP expression and citP mRNA processing. For the citP mRNA processing, citP primers were used to quantify both processed and unprocessed mRNA1 and mRNA2, in which mRNA1 and mRNA2 refer to mRNA produced from promoter $\mathrm{P} 1$ and $\mathrm{P} 2$ of the citQRP operon, respectively (de Felipe et al., 1995).

\begin{tabular}{lll}
\hline Target & & Sequences $\left(\mathbf{5}^{\prime} \rightarrow \mathbf{3}^{\prime}\right)$ \\
\hline recA & Fw primer & ACAACAGTCGCTCTTCATGC \\
& Rv primer & ATTTGAGCCCTTGTTCACC \\
citP (plasmid) & Fw primer & CATTGCTATGCCAATCATGG \\
& Rv primer & TTCCCGAGGATAACTGTTGG \\
& Probe & [TXRD]CACAATACCAGCACCAACTCCACCA[BHQ2] \\
glyA (chromosome) & Fw primer & CAAAAGCAGTATGGCAGCA \\
& Rv primer & ACATCAACCGCTTCTGTTCC \\
& Probe & [6FAM]ACGTTCCCAGGATAACCTTCGGC[BHQ1] \\
Unprocessed mRNA1 & Fw primer & TGAAATTAACTAGCAATTCGGGTA \\
& Rv primer & ACAAAGGCGCGTTAATGTT \\
Unprocessed mRNA1 & Fw primer & CCGAGTGAACGAGGTCAAT \\
and mRNA2 & Rv primer & ACAAAGGCGCGTTTAATGTT \\
\hline
\end{tabular}

The qPCR program started with heating the samples for $5 \mathrm{~min}$ at $95^{\circ} \mathrm{C}$ followed by 39 cycles of 30 seconds at $95^{\circ} \mathrm{C}$ and 30 seconds at $58^{\circ} \mathrm{C}$. Fluorescence was measured at the end of each cycle. After 39 cycles a melting curve was made by a stepwise increase of the temperature from $65^{\circ} \mathrm{C}$ to $95^{\circ} \mathrm{C}$ in steps of $0.5^{\circ} \mathrm{C}$ every 5 seconds. To determine the efficiency of the PCR reaction, a calibration curve was made using a dilution series of isolated DNA. The efficiency did not significantly deviate from 2 for all PCR reactions (mean $\pm S E ; 2.02 \pm 0.04$ and $2.05 \pm 0.08$ ). Therefore, the relative expression was calculated using an efficiency of 2 as stated in equation 4.1 .

$$
\text { Relative expression }=2^{C_{t, r e f}-C_{t, g o i}}
$$

where $\mathrm{Ct}_{\text {,goi }}$ is the threshold cycle of the gene of interest and $\mathrm{Ct}$, ref is the threshold cycle of the reference gene.

Of each sample, RNA was isolated at least in duplicate and for each RNA extraction procedure the gene expression was determined by qPCR in triplicate. The average expression within one RNA extraction procedure was calculated and corrected for 
the measured average plasmid copy number in that specific sample. This corrected expression was used as input for the statistical analysis.

\section{Processing of citP mRNA}

Processing of citP mRNA was analysed by reverse transcriptase qPCR as described for the determination of relative citP expression with the following modifications. Primers were used targeting either $\mathrm{i}$ ) unprocessed mRNA1, ii) unprocessed mRNA1 and mRNA2, or iii) both processed and unprocessed mRNA1 and mRNA2 (total citP mRNA). The primer sequences are given in Table 4.1. The amount of cDNA used in the PCR reactions was decreased from approximately 175 to $87 \mathrm{ng}$. Because the efficiency of the PCR reaction was significantly lower than 2 , relative abundances of the unprocessed mRNAs compared to the total citP mRNA were calculated with equation 4.2.

$$
\text { Relative abundance }=\frac{E_{t}^{C_{t, t}}}{E_{u}^{C_{t, u}}}
$$

where $E_{u}$ and $E_{t}$ are the efficiencies of the $P C R$ reactions using primers for unprocessed mRNA species and total mRNA, respectively, and $\mathrm{Ct}_{\mathrm{u}}$ and $\mathrm{Ct}_{\mathrm{t}}$ are the threshold cycles of unprocessed mRNA species and total mRNA, respectively.

\section{Plasmid copy number determination}

The plasmid copy number was determined in 2 steps: i) extraction of DNA and ii) quantification of chromosomal and plasmid DNA by qPCR. DNA was extracted from bacterial cultures using the DNeasy Blood and tissue kit (Qiagen, Germany). The amount of cells used for the DNA extraction was standardised at a very low amount ( $1 \mathrm{ml}$ of culture with optical density of 0.02 at $600 \mathrm{~nm}$ ) to obtain similar extraction efficiencies for chromosomal and plasmid DNA. The standardised cell suspension was centrifuged for $5 \mathrm{~min}$ at $17000 \times \mathrm{g}$. The pellet was washed with 1 $\mathrm{ml}$ PPS and resuspended in $0.25 \mathrm{ml}$ lysis buffer $(20 \mathrm{mM}$ Tris- $\mathrm{HCl}, 2 \mathrm{mM}$ EDTA, $1.2 \%\left(\mathrm{w} / \mathrm{v}\right.$ ) Triton-X-100, $1 \mathrm{mg}^{\mathrm{m}} \mathrm{ml}^{-1}$ lysozyme and $50 \mathrm{U}$ mutanolysin, $\mathrm{pH}$ 8.0). The suspension was incubated at $37^{\circ} \mathrm{C}$ for 1 hour. Subsequently, $15 \mu$ proteinase $\mathrm{K}$ and $125 \mu \mathrm{AL}$ buffer were added. After incubation at $56^{\circ} \mathrm{C}$ for 1 hour, $125 \mu \mathrm{l}$ absolute ethanol was added. The released DNA was purified using a spin-column provided by the kit. First, the mixture was transferred to the spin-column and centrifuged for $1 \mathrm{~min}$ at $6000 \times \mathrm{g}$. Then $500 \mu \mathrm{l}$ washing buffer AW1 was added and centrifuged for $1 \mathrm{~min}$ at $6000 \times \mathrm{g}$. To remove all traces of buffer, the column was centrifuged for 4 minutes at $17000 \times g$. Finally, the DNA was eluted from the column with elution buffer $\mathrm{AE}$ preheated at $80^{\circ} \mathrm{C}$. Elution buffer was added to the column, incubated for 10 minutes at room temperature and centrifuged for 1 
minute at $6000 \times g$. This step was repeated in total three times. The extracted DNA was stored at $-20^{\circ} \mathrm{C}$ until the plasmid copy number was determined using qPCR.

Chromosomal and plasmid DNA were quantified with qPCR (Bio-Rad Thermal cycler CFX96 Real Time system). For the chromosomal DNA control, the single copy gene glyA was used and for plasmid pLd1 the citP gene was targeted. Taqman probes were used to perform multiplex qPCR reactions, so PCR reactions of glyA and citP took place in a single well in 96-wells plates. Sequences of the used primers and probes are given in Table 4.1. The PCR reaction took place in $20 \mu \mathrm{l}$ and contained $0.2 \mathrm{mM}$ dNTPs, $0.5 \mu \mathrm{M}$ of all four primers, $0.25 \mu \mathrm{M}$ of each probe, $2 \mu \mathrm{l}$ 10x DreamTaq buffer with $\mathrm{MgCl}_{2}$ (Thermo Scientific, USA), $0.8 \mathrm{U}$ DreamTaq DNA polymerase and $2 \mu \mathrm{INA}$. The PCR program started with heating the samples for $5 \mathrm{~min}$ at $95^{\circ} \mathrm{C}$ followed by 39 cycles of 10 seconds at $95^{\circ} \mathrm{C}$ and 20 seconds at $59^{\circ} \mathrm{C}$. Fluorescence of the probes was measured. A threshold of 800 RFU was used to determine the threshold cycle $\left(C_{t}\right)$. An efficiency of 2 was used for both PCR reactions for calculation of the plasmid copy numbers. The following equation was used:

$$
\text { Plasmid copy number }=2^{C_{t, \text { chromosome }}-C_{t, \text { plasmid }}}
$$

From each sample DNA was isolated at least in quadruplicate and for each DNA extraction the PCN was determined by qPCR in sextuplicate. As input for the statistical analyses, the average plasmid copy number of a DNA extraction was taken.

\section{Statistical analysis}

To analyse how significant the effect of the parameters $(\mathrm{pH}$, citrate and nutrient limitation) were on the plasmid copy number, citP expression and the citrate utilisation rate, statistical analyses were conducted in $\mathrm{R}$ (version 3.1 .3 ) using the nlme package (version 3.1-120 (Pinheiro et al., 2016)). Linear mixed-effects modelling was used to correct for the nested structure of the data: different conditions were analysed in the same cultivation, while duplicates of conditions were performed in different cultivations. Therefore, cultivation was used as random categorical variable. First the linear mixed-effects (LME) model with all fixed explanatory variables ( $\mathrm{pH}$, citrate, nutrient limitation and their interactions) was compared with a generalised least square model (GLS) by restricted maximum likelihood estimation (REML) to see which model was better. Subsequently, the fixed explanatory variables of the selected model containing all explanatory variables were optimised by a repetitive process of fitting a full model, dropping all allowable terms in turn, comparing models by maximum likelihood estimation and dropping the least significant term. This process was repeated until 
all terms were significant (Zuur et al., 2009). The residual errors in final model were checked for homogeneity.

Analysis of covariance (ANCOVA) was used to determine whether any of the parameters $(\mathrm{pH}$, citrate and nutrient limitation) had a significant influence on the relation between citP expression and citrate utilisation rate. The average expression and citrate utilisation rate per condition per cultivation was used for the analysis. A linear mixed-effects (LME) model with only citP expression as explanatory variable and cultivation as random categorical variable was compared by $\mathrm{REML}$ with LME models that also included $\mathrm{pH}$, citrate or nutrient limitation as explanatory variable.

\section{Results}

To study the regulation of citrate uptake and metabolism, Lactococcus lactis biovar diacetylactis FM03-V1 was grown in independent duplicate chemostat cultivations at a growth rate of $0.135 \pm 0.004 \mathrm{~h}^{-1}$. In these cultivations the bacterium was subjected to various conditions to study the effect of $\mathrm{pH}$, citrate, nutrient limitation and their interactions on various cellular processes linked to citrate utilisation (Table 4.2). In each condition we analysed the effect on plasmid copy number of pLd1, citP expression, citP mRNA processing, citrate utilisation capacity and metabolite production.

Table 4.2: Overview of the experimental design of the chemostat cultivations. All combinations of nutrient limitation, $\mathrm{pH}$ and citrate have been performed in duplicate in four separate cultivations. The sequences of conditions in these cultivations were as follows: cultivation 1: 1, 4, 2, 3; cultivation 2: 4, 3, 1, 2; cultivation 3 and 4: 7, 8, 5, 6. Parameters other than $\mathrm{pH}$, nutrient limitation and citrate content were kept constant during the cultivations. Bacteria were grown anaerobically at a dilution rate of $0.135 \mathrm{~h}^{-1}$ at $30^{\circ} \mathrm{C}$ with a stirring speed of $300 \mathrm{rpm}$.

\begin{tabular}{llll}
\hline Condition & Nutrient limitation & pH & Citrate \\
\hline 1 & Lactose & 5.5 & + \\
2 & Lactose & 5.5 & - \\
3 & Lactose & 7 & + \\
4 & Lactose & 7 & - \\
5 & Amino acid & 5.5 & + \\
6 & Amino acid & 5.5 & - \\
7 & Amino acid & 7 & + \\
8 & Amino acid & 7 & - \\
\hline
\end{tabular}




\section{Citrate utilisation increases at low pH}

The residual lactose concentration was measured to determine whether the selected media resulted in lactose and amino acid limited conditions indicated by complete or incomplete lactose consumption, respectively. Residual lactose concentrations were below $0.05 \mathrm{mM}$ and above $10 \mathrm{mM}$ for lactose-limited and amino acid-limited media, respectively, which confirmed that growth was limited by either lactose or amino acids. In all conditions with citrate present in the medium, citrate consumption was observed. However, more than $99.7 \%$ was consumed at $\mathrm{pH} 5.5$, while at $\mathrm{pH} 7$ only $30 \%$ and $63 \%$ of the citrate was consumed in lactose and amino acid-limited cultures, respectively. Because the biomass concentration was similar, also the biomass specific citrate utilisation rates were higher at $\mathrm{pH} 5.5$ and under amino acid limitation (Fig. 4.1).

These differences suggested that both an acidic environment and amino acid limitation increased the citrate utilisation capacity. To identify how these parameters regulate the citrate utilisation capacity - by affecting the plasmid copy number, the citP mRNA level, citP mRNA processing or the CitP protein level - the plasmid copy number, the expression of citP, processing of citP mRNA and the citrate utilisation capacity were determined.

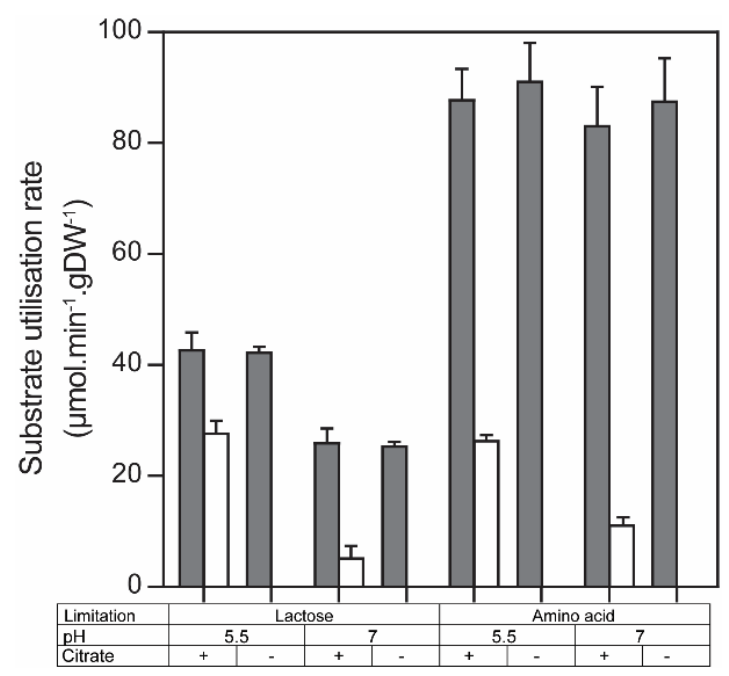

Figure 4.1: Substrate utilisation rates $\left(\mu \mathrm{mol} \cdot \mathrm{min}^{-1} \cdot \mathrm{gDW}^{-1}\right)$ of lactose and citrate during chemostat cultivation at constant growth rate $\left(0.135 \mathrm{~h}^{-1}\right)$ at different conditions: $\mathrm{pH} 5.5$ or 7 , in presence $(+)$ or absence (-) of citrate and under lactose or amino acid limitation. Filled and open bars represent lactose and citrate utilisation, respectively. Error bars represent the standard deviation of two biological replicates. Lactose and citrate were measured in duplicate using HPLC and enzymatic determination, respectively. 


\section{Higher plasmid copy number at low pH and under amino acid limitation}

To determine the plasmid copy number of plasmid pLd1, plasmid and chromosomal DNA were quantified with GPCR and the ratio between them was calculated (Fig. 4.2). We fitted these ratios to linear mixed effect models to find which factors significantly affected the plasmid copy number (see materials and methods). Significant effects were found for $\mathrm{pH}$, nutrient limitation and the interaction between nutrient limitation and pH (Table 4.3). Compared to growth under lactose limitation at $\mathrm{pH} 7$, the plasmid copy number increased both under amino acid limitation (58\%) and at low pH (35\%). These changes in plasmid copy numbers were caused by changes in plasmid replication rates because the growth rate was kept constant.

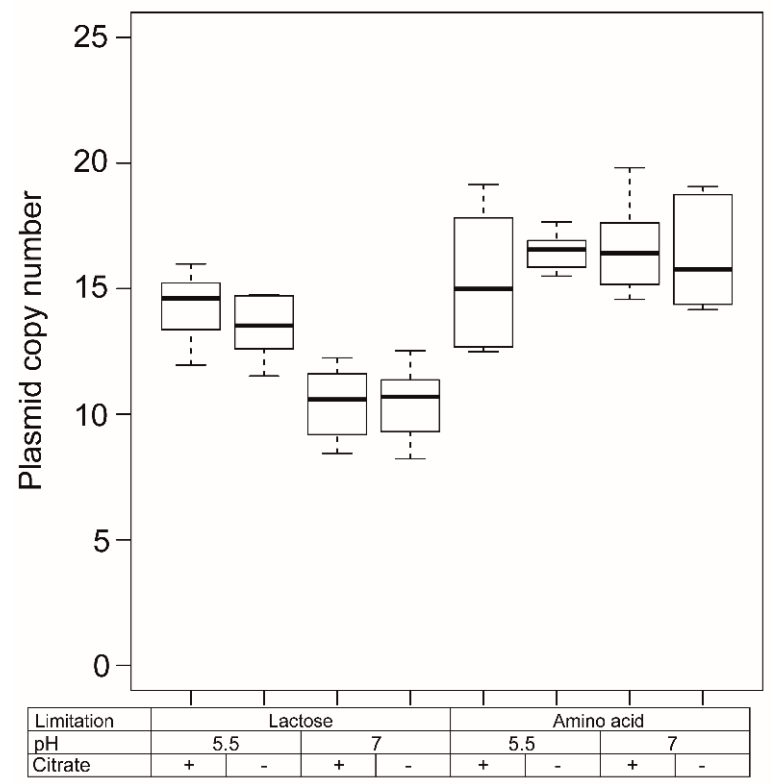

Figure 4.2: Plasmid copy number of plasmid pLd1 of chemostat grown cells cultivated at a constant growth rate $\left(0.135 \mathrm{~h}^{-1}\right)$ at different $\mathrm{pH}$ values, in the presence $(+)$ or absence $(-)$ of citrate and under lactose or amino acid limitation. The single-copy genes citP and glyA were targeted during qPCR to quantify plasmid and chromosomal copies, respectively. 
Table 4.3: Significance of effects of $\mathrm{pH}$, nutrient limitation and the presence of citrate and their interactions on the plasmid copy number (PCN) and relative citP expression. Statistical analysis was performed with $R$ by regression with a linear mixed effect (LME) model as explained in the experimental procedures. Df: degrees of freedom.

\begin{tabular}{|c|c|c|c|c|c|c|}
\hline & \multicolumn{3}{|c|}{ PCN (LME) } & \multicolumn{3}{|c|}{ citP expression (LME) } \\
\hline & $\mathrm{df}$ & $t$ value & $P$ value & df & $t$ value & $P$ value \\
\hline $\mathrm{pH}$ & 53 & -7.53 & $6.2 \times 10^{-10}$ & 28 & -2.3 & 0.029 \\
\hline Nutrient limitation & 2 & 1.16 & 0.37 & & & \\
\hline Citrate & & & & 28 & 4.6 & $9.1 \times 10^{-5}$ \\
\hline $\mathrm{pH}$ :Nutrient limitation & 53 & 4.13 & $3.2 \times 10^{-8}$ & & & \\
\hline pH:Citrate & & & & 28 & -2.5 & 0.018 \\
\hline
\end{tabular}

\section{Both citrate and low $\mathrm{pH}$ are required for high expression of citP}

In addition to changes in the plasmid copy number, citrate uptake is regulated by modulating transcription of the citrate permease gene citP. Relative expression of citP was corrected for the plasmid copy numbers to quantify the effect of $\mathrm{pH}$, citrate and nutrient limitation on transcription of citP (Fig. 4.3).

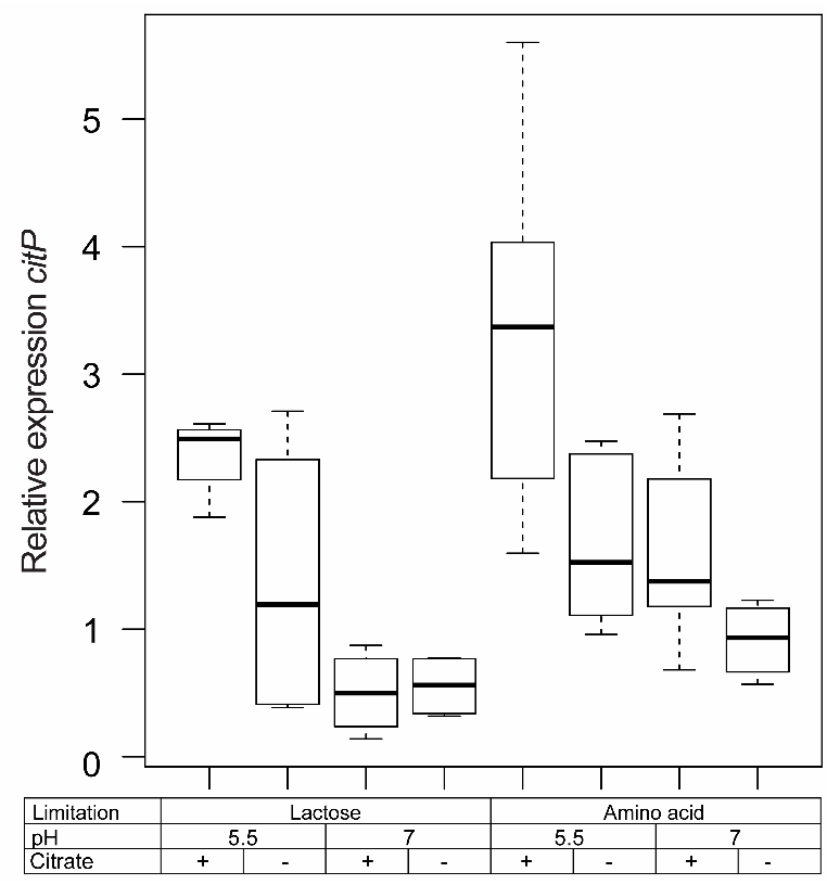

Figure 4.3: Expression of citP relative to the expression of the housekeeping gene recA. Expression has been corrected for the plasmid copy number of pLd1 (Fig. 4.2). Cells have been cultivated in chemostats at a constant growth rate at different $\mathrm{pH}$, in the presence $(+)$ or absence (-) of citrate and under lactose or amino acid limitation. 
Fitting of the data to linear mixed effect models revealed that the $\mathrm{pH}$, citrate and their interaction significantly affected the expression of citP (Table 4.3). After correction for the plasmid copy number, expression of citP was found to be 1.9 and 2.8-fold higher when cells were grown at low pH in the presence of citrate than when cells were grown either at low $\mathrm{pH}$ or in the presence of citrate, respectively. No significant effect of the nutrient limitation on citP expression was found. This indicates that the nutrient limitation only affects the citrate utilisation capacity by increasing the number of copies of plasmid pLd1.

\section{CitP processing is not affected by $\mathrm{pH}$, nutrient limitation or presence of citrate}

The citQRP operon contains promoters $\mathrm{P} 1$ and $\mathrm{P} 2$ resulting in mRNA1 and mRNA2 of $2.9 \mathrm{~kb}$ and $1.9 \mathrm{~kb}$, respectively (de Felipe et al., 1995, 1996, Drider et al., 1998). These mRNAs are processed in a complex secondary structure (Drider et al., 1998). Primers sets has been designed which target specifically i) unprocessed mRNA1, ii) unprocessed mRNA1 and mRNA2 and iii) both processed and unprocessed mRNA1 and mRNA2 (total mRNA; same primers as used for relative citP expression). To determine the effect of the $\mathrm{pH}$, nutrient limitation and the presence of citrate on processing of the citQRP mRNA, the abundance of processed and unprocessed mRNA was analysed with reverse transcriptase qPCR (Fig. 4.4). Surprisingly, the PCR using primers targeting both processed and unprocessed mRNA gave a lower response than the primers targeting only unprocessed mRNA. This could be caused by processing of the mRNA at different sites than reported. Primers targeting unprocessed mRNA1 or both unprocessed mRNA1 and mRNA2 gave almost identical results showing that mRNA1 was the dominant mRNA species and transcription was mainly driven from promoter $\mathrm{P} 1$. No significant differences were found between the various conditions in the abundance of unprocessed mRNA1 and mRNA2 relative to the total mRNA, showing that processing of the mRNAs was not affected by either $\mathrm{pH}$, nutrient limitation or the presence of citrate in the medium. 


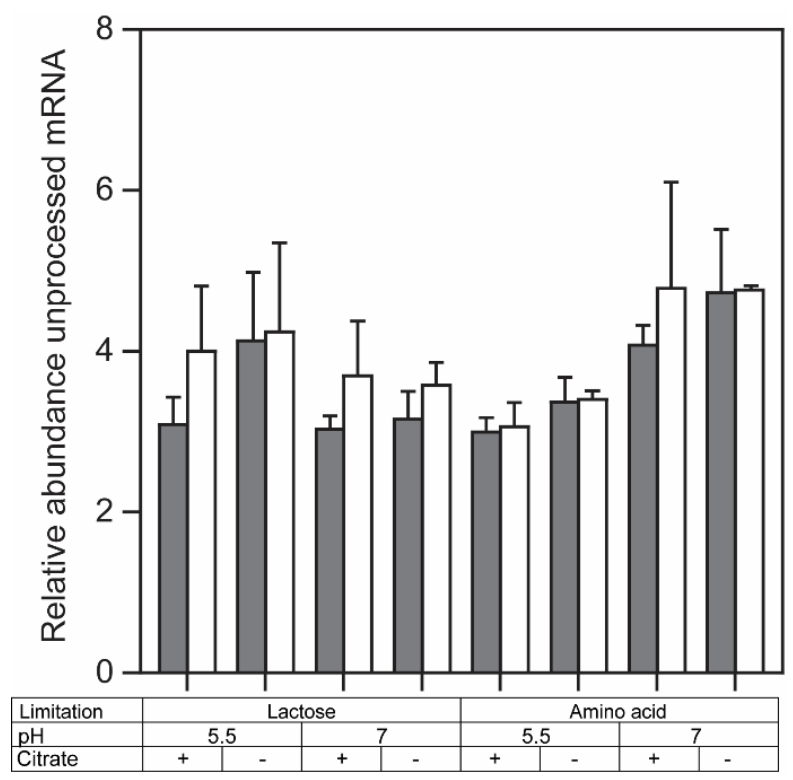

Figure 4.4: Abundance of unprocessed mRNA1 and mRNA2 (filled bars) and unprocessed mRNA1 (open bars) relative to the total citP mRNA (both processed and unprocessed mRNA1 and mRNA2) determined with reverse transcriptase qPCR using primers given in Table 4.3. mRNA1 and mRNA2 refer to mRNA produced from promoter P1 and P2 of the citQRP operon, respectively (de Felipe et al., 1995). Error bars represent the standard deviation of biological duplicates.

\section{Citrate utilisation capacity correlates with citP expression}

Expression of citP was compared to the citrate utilisation rate to find indications of translational and post-translational regulation mechanisms. The citrate utilisation rate was determined in non-growing cells in fresh chemically defined lactose-free medium in which the citrate concentration was monitored in time. Similar trends were found for the citrate utilisation rate as for the expression of citP (Fig. 4.5). Both low pH and the presence of citrate did significantly increase the citrate utilisation rate. The measured utilisation rates were similar to those found in the chemostat cultivation (compare Fig. 4.1 and Fig. 4.5). Analysis of covariance (ANCOVA) showed that none of the tested parameters significantly affected the relation between expression of citP and citrate utilisation rate. Thus, no indication was found that citrate utilisation is regulated at the translational or post-translational level by these parameters. 


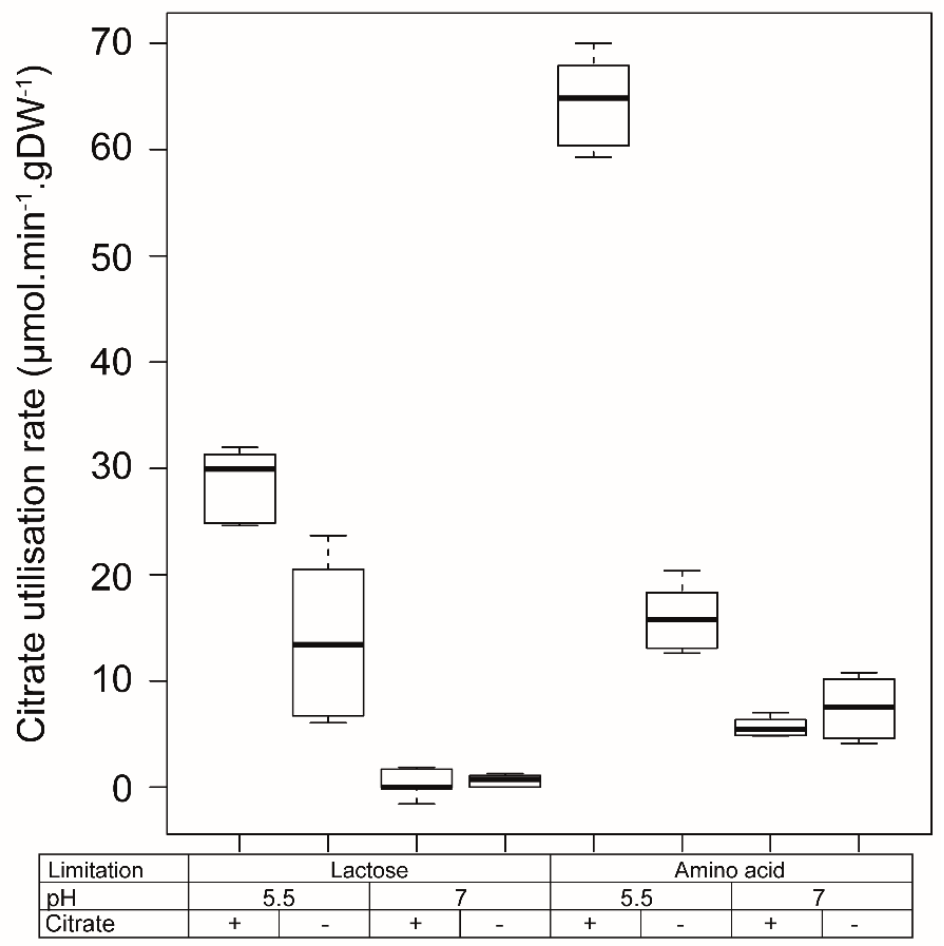

Figure 4.5: Citrate utilisation capacity of chemostat grown cells cultivated at constant growth rate $\left(0.135 \mathrm{~h}^{-1}\right)$ at different $\mathrm{pH}$ values, in the presence $(+)$ or absence $(-)$ of citrate and under lactose or amino acid limitation. The rate of citrate utilisation has been determined and corrected for the biomass concentration.

\section{Acetoin formation increases at low pH}

Although uptake of citrate was shown to be the limiting step in citrate utilisation (Harvey and Collins, 1962), regulation of citrate metabolism could greatly influence the formation of flavour compounds. Metabolite production was analysed to examine the effect of $\mathrm{pH}$, citrate and nutrient limitation on primary metabolism. In all cultivations lactose and citrate were mainly converted into lactate, acetate, formate and ethanol. However, the distribution between the end-products of fermentation differed drastically depending on the cultivation conditions (Fig. 4.6). Lactate was the major end-product (>91 mol\%) under amino acid limitation independent of the $\mathrm{pH}$. This metabolic profile is comparable with the profile found in batch cultures of L. lactis (Starrenburg and Hugenholtz, 1991). Under lactose limitation, only $68 \%$ and $29 \%$ of the end-products was lactate at pH 5.5 and 7, respectively. Instead a mixture of formate, acetate and ethanol was produced. Acetoin was mainly produced at low $\mathrm{pH}$ in the presence of citrate, but in some cases acetoin was also produced in the absence of citrate. This indicates that 
citrate is the main but not the only substrate for acetoin formation. At low $\mathrm{pH}$, the acetoin yield on citrate increased indicating that acetoin formation is regulated by $\mathrm{pH}$ (Fig. 4.7). Citrate is linked to acetoin formation for two reasons: i) citrate is converted into pyruvate without production of reducing equivalents and ii) citrate conversion leads to increased pyruvate production conceivably resulting in higher intracellular pools of pyruvate. Because a-acetolactate synthase has a low affinity for pyruvate $\left(\mathrm{K}_{\mathrm{m}}=50 \mathrm{mM}\right.$ ) (Snoep et al., 1992), a high intracellular pyruvate concentration is required for acetoin formation. The significant export of pyruvate suggests that the intracellular pyruvate concentration was indeed high in conditions where acetoin was formed (Fig. 4.6).

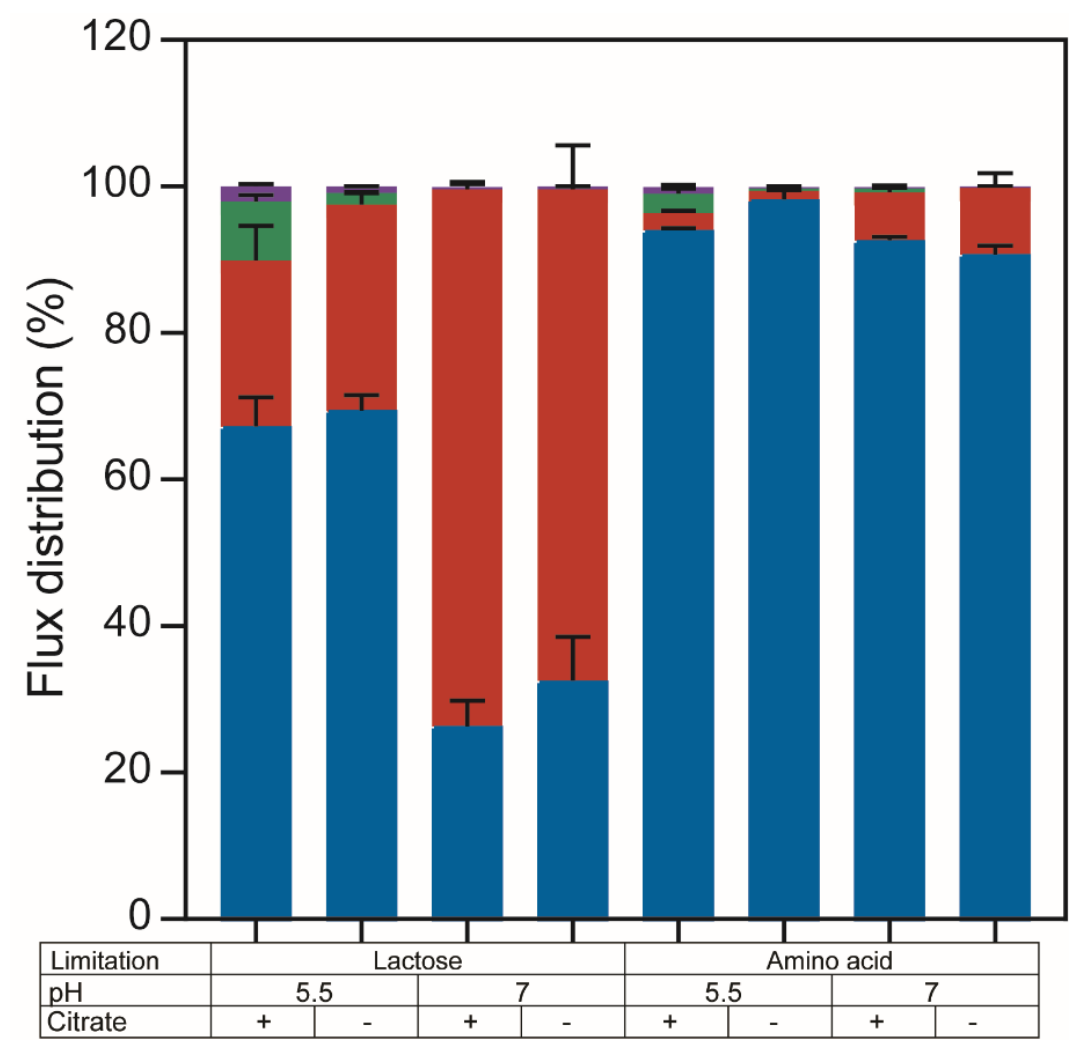

Figure 4.6: Flux distribution of the main metabolic pathways with pyruvate as substrate during chemostat cultivation at constant growth rate $\left(0.135 \mathrm{~h}^{-1}\right)$ at different conditions: $\mathrm{pH}$ 5.5 or 7 , presence $(+)$ or absence (-) of citrate and under lactose or amino acid limitation. Blue: Lactate dehydrogenase; red: pyruvate formate lyase; green: a-acetolactate synthase; purple: pyruvate efflux. It has been taken into account that a-acetolactate synthase converts two pyruvate molecules into one a-acetolactate by multiplying the acetoin production flux by 2 . Error bars represent the standard deviation of two biological replicates. 


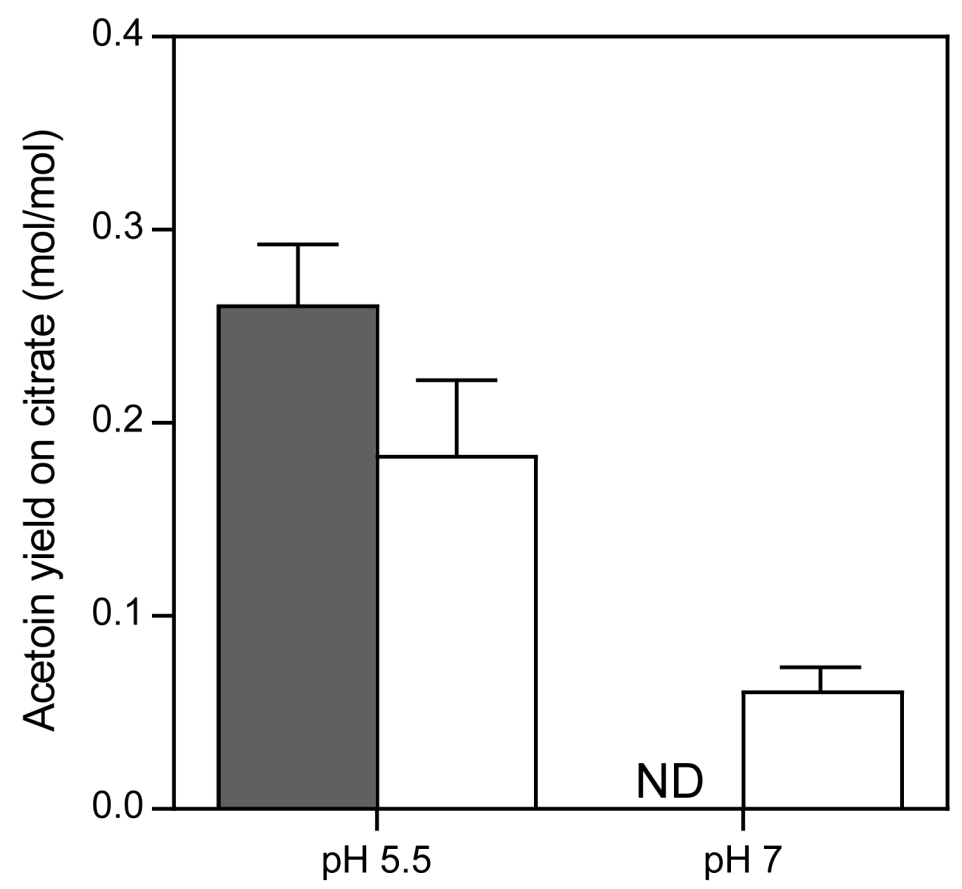

Figure 4.7: Acetoin yield on citrate (mol acetoin / mol citrate) during chemostat cultivation as function of $\mathrm{pH}$ and nutrient limitation. Filled bar: lactose limitation, Open bars: amino acid limitation. ND, not detected. Error bars represent the standard deviation of two biological replicates.

\section{Discussion}

The ability of Lactococcus lactis subsp. lactis biovar diacetylactis to metabolise citrate is an important trait for the dairy industry. In this bacterium citrate metabolism is limited by the uptake of citrate through the citrate permease CitP (Harvey and Collins, 1962). It is known that CitP is induced at low pH (GarcíaQuintáns et al., 1998), but the role of citrate is still under debate (de Felipe et al., 1996, Harvey and Collins, 1962, Magni et al., 1994, Smith et al., 1992). Moreover, the role of the plasmid copy number in regulating the citrate utilisation capacity has never been studied. To fill these knowledge gaps, we studied L. lactis chemostat cultures at different conditions with the objective to elucidate the role of $\mathrm{pH}$, citrate and nutrient limitation in regulation of citrate utilisation. Our study revealed that amino acid limitation significantly increased the plasmid copy number of pLd1. Expression of citP was higher at low pH, but also the supply of citrate in the medium significantly increased transcription. An overview summarising all factors and their interactions affecting citrate utilisation is presented (Fig. 4.8). 


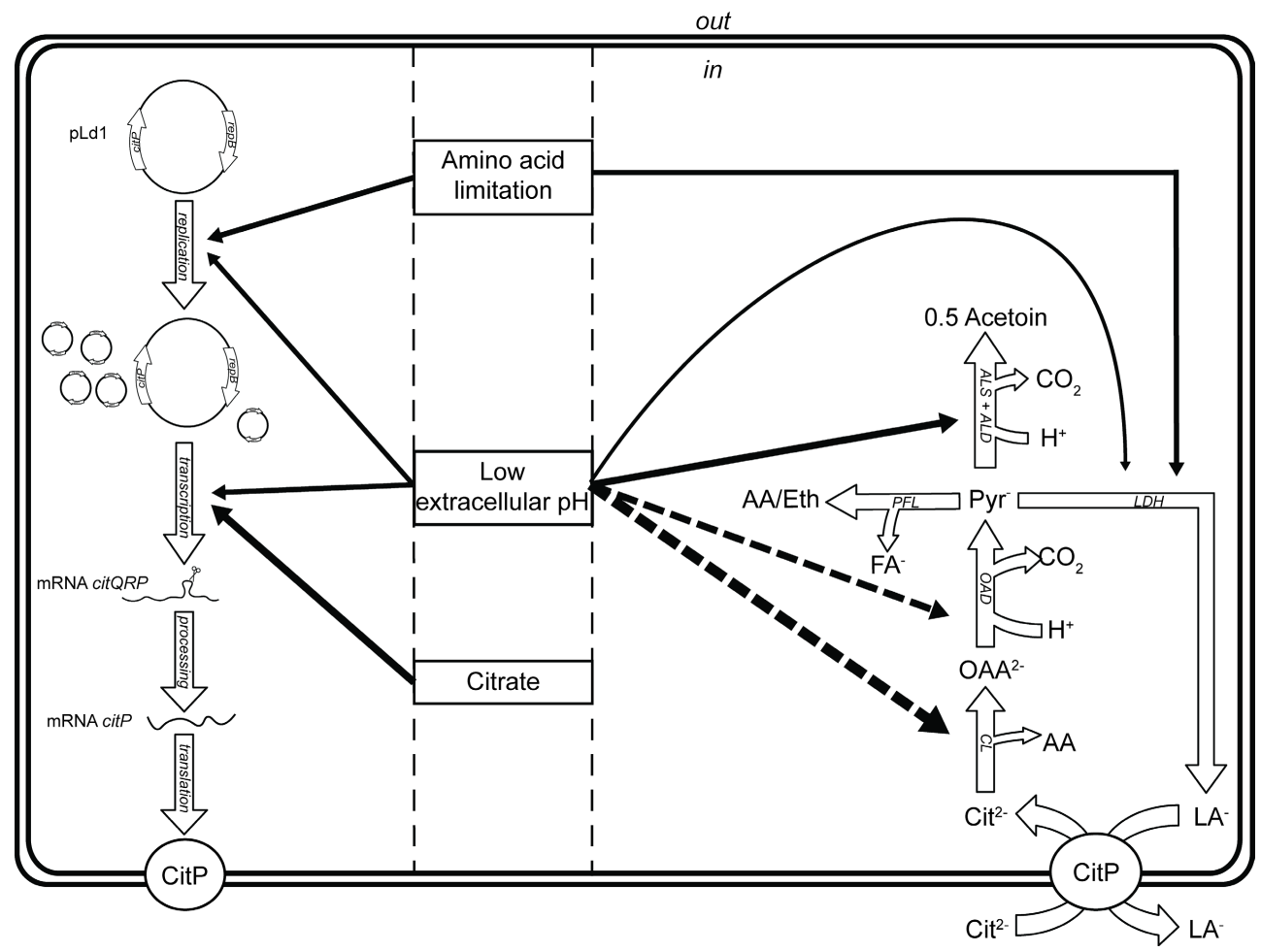

Figure 4.8: Overview of the regulation of citrate uptake and metabolism in Lactococcus lactis biovar diacetylactis by the three investigated parameters: $\mathrm{pH}$, citrate and nutrient limitation. Filled arrows represent a positive influence on the process or reaction. Dashed arrows represent effects found in other studies (Martín et al., 2004). Thicker arrows represent bigger effects. On the left side regulation of citP expression and translation is summarised showing regulation at the level of the plasmid copy number of pLd1 and citP expression. On the right side regulation of citrate metabolism is shown. Low pH increases acetoin production via induction of citrate lyase and the acetoin forming pathway. Low $\mathrm{pH}$ also stimulates homolactic fermentation compared with mixed acid fermentation. Amino acid limitation mainly results in lactic acid production. Abbreviations: $\mathrm{Cit}^{2-}$ : divalent citrate; $\mathrm{LA}^{-}$: lactate; $\mathrm{FA}^{-}$: formate; $\mathrm{OAA}^{-}$: oxaloacetate; Pyr': pyruvate; $\mathrm{AA}$ : acetic acid; Eth: ethanol; $\mathrm{CL}$ : citrate lyase; OAD: oxaloacetate decarboxylase; ALS: a-acetolactate synthase; ALD: aacetolactate decarboxylase; PFL: pyruvate formate lyase; LDH: lactate dehydrogenase.

The plasmid copy number of pLd1 increased approximately 1.5 -fold in cells cultivated at low pH or under amino acid limitation. The higher copy number can be ascribed to elevated plasmid replication rates because the growth rate was constant. The amino acid sequence of the replication initiation protein RepB revealed that plasmid pLd1 replicates via a theta type mechanism (del Solar et al., 1998). At the complementary strand of the rep $B$ gene, a small putative counter-transcript RNA (ctRNA) is encoded, which is located on the 5 ' -end of the 
repB coding sequence (Suppl. Fig. S4.1). This ctRNA is similar to the ctRNA of pND324 (Duan et al., 1998) and might also be involved in inhibition of translation of rep $B$ by interacting with repB mRNA. By this mechanism the ctRNA could control the plasmid copy number. The role of $\mathrm{pH}$ and nutrient limitation in this regulation mechanism remains to be elucidated.

In addition to the effect of plasmid pLd1 copy number, we confirmed that citP is induced at low pH (García-Quintáns et al., 1998). Moreover, our study demonstrates that citP expression is higher in cells when citrate is present during growth and that this correlates with higher citrate utilisation rates. The increased citrate utilisation capacity for cells grown in the presence of citrate was also found by Harvey and Collins (1962). However, Magni et al. (1994) and Smith et al. (1992) did not find an effect on the citrate uptake rate by supplementing M17 growth medium with citrate. We demonstrated by enzymatic determination of citrate that $\mathrm{M} 17$ already contains $0.80 \pm 0.07 \mathrm{mM}$ citrate, which probably results in induction of citP. The presence of citrate in M17 has been overlooked by many researchers (de Felipe et al., 1995, García-Quintáns et al., 1998, Magni et al., 1994, Magni et al., 1999, Martín et al., 2004, Smith et al., 1992) and this most likely explains why those researchers did not find any effect of citrate on the regulation of citrate uptake and metabolism in L. lactis biovar diacetylactis.

In various heterofermentative bacteria citrate does play a role in regulation of citrate uptake and metabolism. In Weissella paramesenteroides, citrate induces the cit operon via the transcriptional activator CitI (Martín et al., 2005). In addition to induction of the citI gene by the presence of citrate in the medium, citrate increases the DNA-binding affinity of CitI to its two operator sites enhancing recruitment of RNA polymerase at the promoters PcitI and Pcit (Martín et al., 2005). In L. lactis, citI is induced at low pH (Martín et al., 2004). However, the effect of citrate on citI induction remains to be elucidated because all experiments in that particular study were performed with M17 grown cells. The promoter of citI in L. lactis contains an operator site for binding of CitI (Martín et al., 2005), and we speculate that the presence of citrate affects citrate uptake by modulating expression of citI. In promoter $\mathrm{P} 1$, which mainly drives citP expression, we found a putative operator site for CitI (Suppl. Fig S4.2). This operator site is located 121 bp upstream of the transcription start site and might be involved in regulation of citP by citrate via CitI.

Induction of promoter $\mathrm{P} 1$ results in a polycistronic mRNA containing citQ, citR and citP (de Felipe et al., 1995, 1996). CitQ and CitR are involved in posttranscriptional regulation of CitP by processing the mRNA in a complex secondary structure (de Felipe et al., 1995, Drider et al., 1999). None of the tested 
parameters in this study significantly affected the processing of the mRNA or the relation between citP expression and the citrate utilisation rate. This suggests that citrate utilisation was not regulated at the post-transcriptional level by any of these parameters. However, the amount of CitP protein has to be quantified to confirm this.

Although transport of citrate has been reported to be limiting (Harvey and Collins, 1962), enzymes responsible for citrate metabolism, i.e. citrate lyase and oxaloacetate decarboxylate, could become limiting under specific conditions. The genes encoding these enzymes are located on the chromosome in the citMcitCDEFXG operon. Like the citQRP operon, this operon is induced at low $\mathrm{pH}$ resulting in increased activities of citrate lyase and oxaloacetate decarboxylase at low pH (Martín et al., 2004, Sender et al., 2004). Therefore, the very low citrate utilisation rate at $\mathrm{pH} 7$ under lactose limitation in which citP is expressed could be caused by low activities of citrate lyase or oxaloacetate decarboxylase.

In addition to regulating citrate uptake, $\mathrm{pH}$ plays a role in the metabolism of citrate. Both citrate lyase and a-acetolactate synthase have been shown to be induced in acidic environments (García-Quintáns et al., 2008, Martín et al., 2004). These enzymes convert citrate via pyruvate into C4-compounds acetoin and diacetyl. Therefore, induction of the genes encoding these enzymes most likely caused the increased acetoin yield at acidic conditions. Knowing the mechanisms governing citrate utilisation in Lactococcus lactis biovar diacetylactis will help to select conditions to improve flavour formation from citrate.

\section{Nucleotide sequence accession numbers}

The sequence of plasmid pLd8, pLd9, pLd10, pLd11 and pLd12 of Lactococcus lactis FM03-V1 has been deposited at GenBank under the accession numbers MF150536, MF150537, MG813924, MG813925 and MG813926.

\section{Acknowledgments}

This work was financially supported by Arla Foods (Aarhus, Denmark). The authors would like to acknowledge Judith Wolkers-Rooijackers for her technical support with the HPLC analysis. 


\section{Supplementary materials}

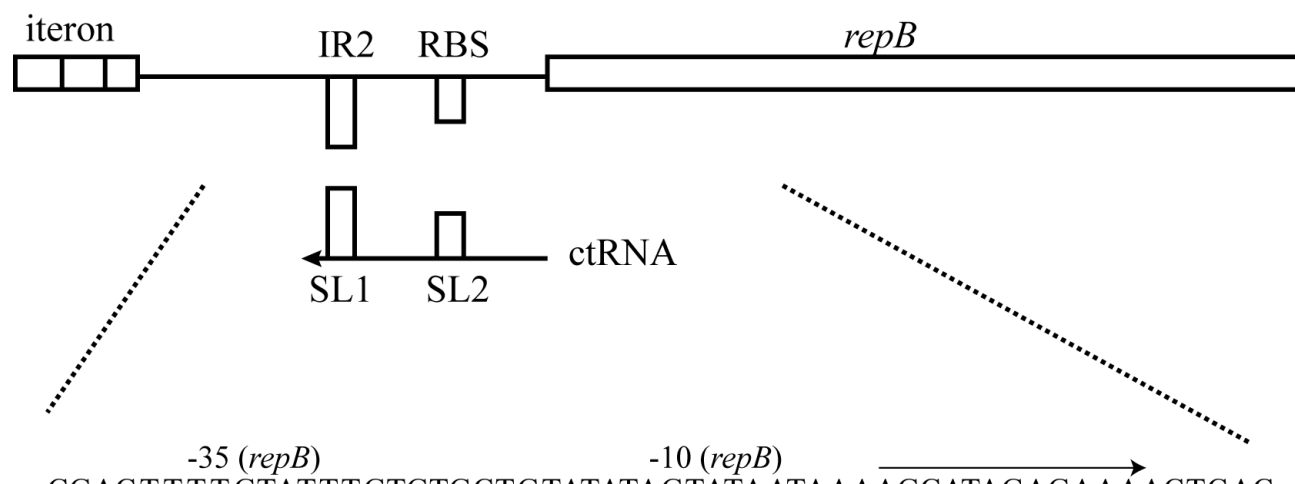

CGACTTTTGTATTTGTGTGCTGTATATAGTATAATAAAAGCATAGAGAAAACTCAC GCTGAAAACATAAACACACGACATATATCATATTATTTTCGTATCTCTTTTGAGTG

IR2

RBS

TATGAAATGACTTTCTCTATGCTACTACTAAAACACGCAAAGGAGCGTATTTATACT ATACTTTACTGAAAGAGATACGATGATGATTTTGTGCGTTTCCTCGCATAAATATGA $+1$

$\rightarrow \operatorname{rep} B$

ATGATTATACCAGAAAAACAAAACAAACAAAAGCAGG

TACTAATATGGTCTT TTTGTTTTGTTTGTTTTCGTCC -10 (ctRNA)

-35 (ctRNA)

Supplementary Figure S4.1: Genetic organisation of the replicon of plasmid pLd1. Underlined nucleotides are -35 and -10 signals, the putative ribosome binding site (RBS) and the startcodon for $\operatorname{rep} B$ (thick arrow). The two thin arrows indicate the inverted repeat IR2. The picture has been designed according to plasmid pND324 (Duan et al., 1998). Iteron: 2.9 times 22-bp direct repeats; SL1 and SL2: stem-loop structures in ctRNA.

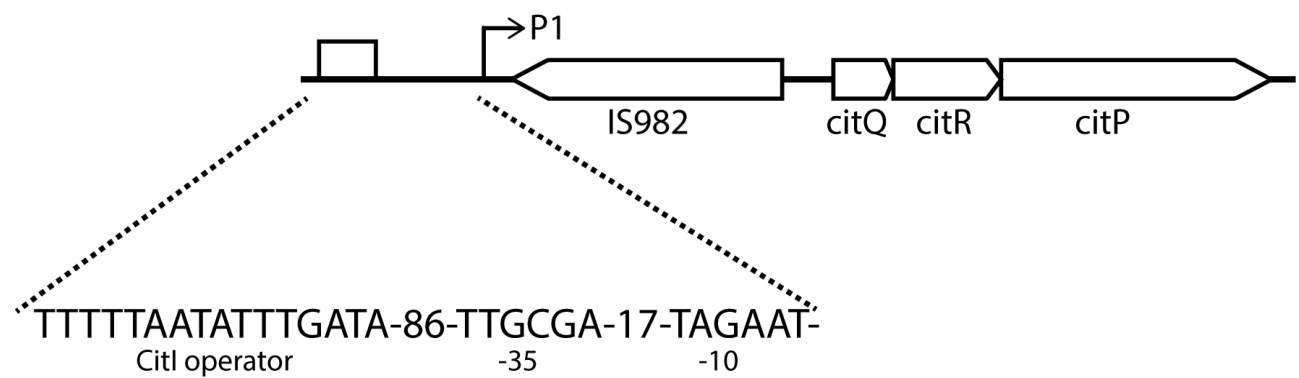

Supplementary Figure S4.2: Schematic representation of citQRP operon in Lactococcus lactis subsp. lactis biovar diacetylactis FM03-V1 with the putative CitI operator site. The arrow indicates the P1 promoter. The nucleotide sequence of the CitI operator site and the -10 and -35 signals are given together with the number of nucleotides between them. 


\section{References}

Bandell, M., Lhotte, M. E., Marty-Teysset, C., Veyrat, A., Prevost, H., Dartois, V., Divies, C., Konings, W. N. and Lolkema, J. S. (1998). Mechanism of the citrate transporters in carbohydrate and citrate cometabolism in Lactococcus and Leuconostoc species. Appl. Environ. Microbiol. 64(5): 1594-1600.

Cachon, R., Daniel, S. and Diviès, C. (1995). Proton-dependent kinetics of citrate uptake in growing cells of Lactococcus lactis subsp lactis bv diacetylactis. FEMS Microbiol. Lett. 131(3): 319-323.

Cogan, T. M. (1981). Constitutive nature of the enzymes of citrate metabolism in Streptococcus lactis subsp. diacetylactis. J. Dairy Res. 48(3): 489-495.

Collins, E. B. (1972). Biosynthesis of flavor compounds by microorganisms. J. Dairy Sci. 55(7): 1022-1028.

de Felipe, F. L., Magni, C., de Mendoza, D. and López, P. (1995). Citrate utilization gene cluster of the Lactococcus lactis biovar diacetylactis: organization and regulation of expression. Mol. Gen. Genet. 246(5): 590-599.

de Felipe, F. L., Magni, C., de Mendoza, D. and López, P. (1996). Transcriptional activation of the citrate permease $P$ gene of Lactococcus lactis biovar diacetylactis by an insertion sequence-like element present in plasmid pCIT264. Mol. Gen. Genet. 250(4): 428-436.

del Solar, G., Giraldo, R., Ruiz-Echevarría, M. J., Espinosa, M. and Díaz-Orejas, R. (1998). Replication and control of circular bacterial plasmids. Microbiol. Mol. Biol. Rev. 62(2): 434-464.

Drider, D., Santos, J. M., Arraiano, C. M. and Lopez, P. (1998). RNA processing is involved in the post-transcriptional control of the citQRP operon from Lactococcus lactis biovar diacetylactis. Mol. Gen. Genet. 258(1-2): 9-15.

Drider, D., Santos, J. M., Garcia-Quintáns, N., Arraiano, C. M. and López, P. (1999). The role of Escherichia coli RNase E and RNase III in the processing of the citQRP operon mRNA from Lactococcus lactis biovar diacetylactis. J. Mol. Microbiol. Biotechnol. 1(2): 337-346.

Drider, D., Bekal, S. and Prevost, H. (2004). Genetic organization and expression of citrate permease in lactic acid bacteria. Genet. Mol. Res. 3(2): 273-281.

Duan, K., Liu, C. Q., Supple, S. and Dunn, N. W. (1998). Involvement of antisense RNA in replication control of the lactococcal plasmid pND324. FEMS Microbiol. Lett. 164(2): 419-426.

García-Quintáns, N., Magni, C., de Mendoza, D. and López, P. (1998). The citrate transport system of Lactococcus lactis subsp. lactis biovar diacetylactis is induced by acid stress. Appl. Environ. Microbiol. 64(3): 850-857.

García-Quintáns, N., Repizo, G., Martín, M., Magni, C. and López, P. (2008). Activation of the diacetyl/acetoin pathway in Lactococcus lactis subsp. lactis bv. diacetylactis CRL264 by acidic growth. Appl. Environ. Microbiol. 74(7): 1988-1996.

Harvey, R. J. and Collins, E. B. (1961). Role of citritase in acetoin formation by Streptococcus diacetilactis and Leuconostoc citrovorum. J. Bacteriol. 82(6): 954-959.

Harvey, R. J. and Collins, E. B. (1962). Citrate transport system of Streptococcus diacetilactis. J. Bacteriol. 83: 1005-1009.

Hugenholtz, J. (1993). Citrate metabolism in lactic acid bacteria. FEMS Microbiol. Rev. 12(13): $165-178$.

Kempler, G. M. and Mckay, L. L. (1979a). Characterization of plasmid deoxyribonucleic acid in Streptococcus lactis subsp. diacetylactis: evidence for plasmid-linked citrate utilization. Appl. Environ. Microbiol. 37(2): 316-323.

Kempler, G. M. and Mckay, L. L. (1979b). Genetic evidence for plasmid-linked lactose metabolism in Streptococcus lactis subsp diacetylactis. Appl. Environ. Microbiol. 37(5): 1041-1043.

Kempler, G. M. and Mckay, L. L. (1981). Biochemistry and genetics of citrate utilization in Streptococcus lactis ssp diacetylactis. J. Dairy Sci. 64(7): 1527-1539. 
Magni, C., de Felipe, F. L., Sesma, F., López, P. and de Mendoza, D. (1994). Citrate Transport in Lactococcus-Lactis Biovar Diacetylactis - Expression of the Plasmid-Borne Citrate Permease-P. FEMS Microbiol. Lett. 118(1-2): 75-82.

Magni, C., de Mendoza, D., Konings, W. N. and Lolkema, J. S. (1999). Mechanism of citrate metabolism in Lactococcus lactis: resistance against lactate toxicity at low pH. J. Bacteriol. 181(5): 1451-1457.

Martín, M. G., Sender, P. D., Peirú, S., de Mendoza, D. and Magni, C. (2004). Acid inducible transcription of the operon encoding the citrate lyase complex of Lactococcus lactis biovar diacetylactis CRL264. J. Bacteriol. 186(17): 5649-5660.

Martín, M. G., Magni, C., de Mendoza, D. and López, P. (2005). CitI, a transcription factor involved in regulation of citrate metabolism in lactic acid bacteria. J. Bacteriol. 187(15): 5146-5155.

Marty-Teysset, C., Lolkema, J. S., Schmitt, P., Divies, C. and Konings, W. N. (1995). Membrane potential-generating transport of citrate and malate catalyzed by Citp of Leuconostoc mesenteroides. J. Biol. Chem. 270(43): 25370-25376.

Marty-Teysset, C., Posthuma, C., Lolkema, J. S., Schmitt, P., Divies, C. and Konings, W. N. (1996). Proton motive force generation by citrolactic fermentation in Leuconostoc mesenteroides. J. Bacteriol. 178(8): 2178-2185.

Pinheiro, J., Bates, D., DebRoy, S., Sarkar, D. and Team, R. C. (2016). nlme: Linear and Nonlinear mixed-effects models. from http://CRAN.R-project.org/package=nlme

Sender, P. D., Martín, M. G., Peirú, S. and Magni, C. (2004). Characterization of an oxaloacetate decarboxylase that belongs to the malic enzyme family. FEBS Lett. 570(1-3): 217-222.

Smith, M. R., Hugenholtz, J., Mikoczi, P., Deree, E., Bunch, A. W. and Debont, J. A. M. (1992). The stability of the lactose and citrate plasmids in Lactococcus lactis subsp lactis biovar diacetylactis. FEMS Microbiol. Lett. 96(1): 7-11.

Snoep, J. L., Demattos, M. J. T., Starrenburg, M. J. C. and Hugenholtz, J. (1992). Isolation, characterization, and physiological role of the pyruvate dehydrogenase complex and alpha-acetolactate synthase of Lactococcus lactis subsp. lactis bv. diacetylactis. J. Bacteriol. 174(14): 4838-4841.

Starrenburg, M. J. C. and Hugenholtz, J. (1991). Citrate fermentation by Lactococcus and Leuconostoc spp. Appl. Environ. Microbiol. 57(12): 3535-3540.

Terzaghi, B. E. and Sandine, W. E. (1975). Improved medium for lactic streptococci and their bacteriophages. Appl. Microbiol. 29(6): 807-813.

van Mastrigt, O., Abee, T. and Smid, E. J. (2017). Complete genome sequences of Lactococcus lactis subsp. lactis bv. diacetylactis FM03 and Leuconostoc mesenteroides FM06 isolated from cheese. Genome Announc. 5(28): e00633-17.

Zuur, A. F., Ieno, E. N., Walker, N. J., Saveliev, A. A. and Smith, G. M. (2009). Mixed effects models and extensions in ecology with $R$. New York: Springer. 



\section{Chapter 5}

\section{Aroma formation during cheese ripening is best resembled by Lactococcus lactis retentostat cultures}

Oscar van Mastrigt, Diego Gallegos Tejeda, Mette. N. Kristensen, Tjakko Abee, Eddy J. Smid

Published in Microbial Cell Factories, July 2018, 17(1):104 Published online: 4 July 2018, doi: 10.1186/s12934-018-0950-7 


\section{Abstract}

Cheese ripening is a complex, time consuming and expensive process, which involves the generation of precursors from carbohydrates, proteins and fats and their subsequent conversion into a wide range of compounds responsible for the flavour and texture of the cheese. This study aims to investigate production of cheese aroma compounds outside the cheese matrix that could be applied for instance as food supplements in dairy or non-dairy products. In this study, aroma formation by a dairy Lactococcus lactis was analysed as a function of the growth medium (milk, hydrolysed micellar casein isolate (MCI) and chemically defined medium (CDM)) and the cultivation conditions (batch culture, retentostat culture and a milli-cheese model system). In the retentostat cultures, the nutrient supply was severely restricted resulting in low growth rates $\left(\sim 0.001 \mathrm{~h}^{-1}\right)$, thereby mimicking cheese ripening conditions in which nutrients are scarce and bacteria hardly grow. In total 82 volatile organic compounds were produced by the bacteria. Despite the use of a chemically defined medium, retentostat cultures had the biggest qualitative overlap in aroma production with the milli-cheese model system ( 36 out of 54 compounds). In the retentostat cultures, 52 known cheese compounds were produced and several important cheese aroma compounds and/or compounds with a buttery or cheese-like odour increased in retentostat cultures compared to batch cultures and milli-cheeses, such as esters, methyl ketones, diketones and unsaturated ketones. In cultures on CDM and MCI, free fatty acids and their corresponding degradation products were underrepresented compared to what was found in the milli-cheeses. Addition of a mixture of free fatty acids to CDM and MCI could help to enhance flavour formation in these media, thereby even better resembling flavour formation in cheese. This study demonstrates that retentostat cultivation is the preferred method to produce cheese flavours outside the cheese matrix by mimicking the slow growth of bacteria during cheese ripening. 


\section{Introduction}

Ripening of cheese is a complex process in which the typical cheese characteristics, such as the flavour and texture, are formed by the action of numerous enzymes derived from the milk, the rennet, the starter bacteria and the non-starter bacteria (Fox et al., 2017). It primarily involves the generation of precursors from carbohydrates, proteins and fats and their conversion into a wide range of compounds responsible for the flavour and texture of the cheese (Smid and Kleerebezem, 2014).

Cheese ripening is a slow and expensive process that can last from about two weeks, e.g. Mozzarella, to more than two years, e.g. Parmigiano-Reggiano (McSweeney, 2004). Consequently, there is a demand to accelerate this complex process while maintaining the flavour balance. Most of the methods to accelerate cheese ripening focus on enhancing proteolysis and lipolysis by for instance ripening at elevated temperatures, the addition of enzymes (e.g. lipases, proteinases and peptidases), microencapsulation of enzymes, using attenuated starter cultures, culture adjuncts and genetically modified cultures. Each of these methods have their advantages and disadvantages that are extensively reviewed by Azarnia et al. (2006), but have in common that they mainly enhance precursor formation and not their subsequent conversion into aroma compounds which requires intact cells with functional metabolic pathways (Smid and Kleerebezem, 2014).

From both a scientific and a technological perspective, it could be interesting to study aroma formation outside the cheese matrix. Such a study could provide new insights into for instance the origin of particular aroma compounds and how their formation is regulated. Moreover, such studies could help to optimise or steer aroma formation by lactic acid bacteria in such a way that these aroma compounds can be applied as food supplements in dairy or dairy-like products.

It is important to realise that during the ripening of cheese the bacteria hardly grow, and this has been shown to affect the aroma formation (van de Bunt et al., 2014, van Mastrigt et al., 2018a). Slow growth in the cheese matrix could be mimicked using retentostat cultivation, which is a modification of the chemostat cultivation in which the biomass is retained in the bioreactor using a filter in the effluent line (Ercan et al., 2015). However, milk cannot be used as medium in retentostat cultures due to clogging of the filter. Therefore, retentostat cultivations were carried out using a chemically defined medium with lactose, citrate and Bacto-tryptone as main carbon and nitrogen sources to mimic the 
composition of milk as much as possible within the constraints imposed by the retentostat cultivation method.

This study aimed at comparing aroma formation by the dairy Lactococcus lactis subsp. lactis biovar diacetylactis FM03-V1 during (i) growth in retentostat cultures using a chemically defined medium, (ii) in batch cultures using a chemically defined medium, hydrolysed micellar casein isolate (MCI) and milk, and (iii) in a milli-cheese model system (Spus et al., 2017).

\section{Materials and methods}

\section{Strain and pre-culture conditions}

Lactococcus lactis subsp. lactis biovar diacetylactis FM03-V1 (van Mastrigt et al., 2017, van Mastrigt et al., 2018b), which has been isolated from a 10-week-old Sams $\varnothing$ cheese, was used in this study. For each cultivation, L. lactis was streaked on M17 plates (Oxoid, UK) supplemented with $0.5 \%(w / v)$ lactose (LM17) and incubated for $48 \mathrm{~h}$ at $30^{\circ} \mathrm{C}$. A single colony was inoculated into appropriate liquid medium (chemically defined medium, hydrolysed MCI or milk) and incubated overnight at $30^{\circ} \mathrm{C}$. These overnight cultures were used to prepare the millicheeses and to inoculate the same media for the batch and retentostat cultivations.

\section{Medium}

\section{Chemically defined medium (CDM)}

The chemically defined media contained $0.5 \%(\mathrm{w} / \mathrm{w})$ lactose, $0.24 \%(\mathrm{w} / \mathrm{w})$ $\left(\mathrm{NH}_{4}\right)_{3}$ citrate and $1 \%(\mathrm{w} / \mathrm{w})$ Bacto-Tryptone as main carbon and nitrogen sources (van Mastrigt et al., 2018b) and the $\mathrm{pH}$ was standardised to 5.5 with $\mathrm{HCl}$. To increase the buffering capacity the phosphate content was increased from 2.67 to $6 \mathrm{~g} / \mathrm{kg} \mathrm{K \textrm {K } _ { 2 }} \mathrm{PO}_{4}$ and from 0 to $6 \mathrm{~g} / \mathrm{kg} \mathrm{K}_{2} \mathrm{HPO}_{4}$ for batch cultivations.

Hydrolysed micellar casein isolate

Micellar casein isolate (MCI) powder was obtained from Arla Foods (Aarhus, Denmark) and stored as powder at $4^{\circ} \mathrm{C}$ until use. The MCI powder was dissolved in demineralised water to a protein content of $5.2 \%$. Subsequently, the solution was heated to $92^{\circ} \mathrm{C}$ for $45 \mathrm{~min}$ and cooled down to $50^{\circ} \mathrm{C}$. The $\mathrm{pH}$ was adjusted to 8 with $6 \mathrm{M} \mathrm{NaOH}$ and $0.018 \%$ Alcalase $\AA 2.4$ L FG (Novozymes, Denmark) was added. After incubation at $50^{\circ} \mathrm{C}$ until the $\mathrm{pH}$ reached 7 , a solution of $6 \mathrm{M} \mathrm{NaOH}$ was used to adjust the $\mathrm{pH}$ to 7 if required and $0.018 \%$ Neutrase $0.8 \mathrm{~L}$ (Novozymes, Denmark) was added. After incubation for 4 hours, enzymes were inactivated at 
$85^{\circ} \mathrm{C}$ for 15 minutes. Subsequently, $0.027 \%$ Flavourzymes $1000 \mathrm{~L}$ (Novozymes, Denmark) was added and the MCI solution was incubated for 18 hours at $50^{\circ} \mathrm{C}$. Finally, enzymes were inactivated at $85^{\circ} \mathrm{C}$ for 15 minutes. After centrifugation for 1 hour at $10^{\circ} \mathrm{C}$ at $15600 \times g$, the hydrolysed MCI was sterilised by filtration through a $0.2 \mu \mathrm{m}$ filter (Millipore, USA).

\section{Cultivations}

\section{Batch cultures}

L. lactis was incubated in at least 5 independent batch cultures at $30^{\circ} \mathrm{C}$ for 2 weeks. Three types of medium were used: full fat UHT milk, hydrolysed MCI and CDM. For the milk pre-cultures, skimmed UHT milk supplemented with $1 \%(\mathrm{w} / \mathrm{w})$ Bacto-tryptone was used.

\section{Retentostat cultures}

Two independent retentostat cultivations were performed in bioreactors with a 1 $\mathrm{L}$ working volume (Infors HT, Switzerland). The stirring speed was set at $400 \mathrm{rpm}$, the $\mathrm{pH}$ was controlled at 5.5 by automatic addition of $5 \mathrm{M} \mathrm{NaOH}$ and the temperature was kept at $30^{\circ} \mathrm{C}$. The headspace was flushed with nitrogen gas at a flow of $0.1 \mathrm{~L} / \mathrm{min}$ to maintain anaerobic conditions. After running the bioreactor for at least 5 volume changes in a chemostat mode at a dilution rate of $0.05 \mathrm{~h}^{-1}$, a polyethersulfone crossflow filter (Spectrum laboratories, USA) was connected to the effluent line in an outer loop to start the retentostat cultivation. Samples were taken every 3 to 4 days, and the aroma profile was compared with the other cultures after 2 weeks.

\section{Milli-cheese}

Milli-cheeses were made with pasteurised full-fat milk (Jumbo, Netherlands) according to Spus et al. (2017) with some adaptations. 1\% (w/w) Bacto-tryptone was added to the milk so no caseinolytic strain had to be added. Forty-five millilitres milk was pre-heated at $30.5^{\circ} \mathrm{C}$ and $5 \mathrm{ml} 10 \%$ Bacto-tryptone, $12.5 \mu \mathrm{l}$ rennet, $20 \mu \mathrm{l}$ of $33 \%\left(\mathrm{w} / \mathrm{v}\right.$ ) $\mathrm{CaCl}_{2}$ and $0.5 \mathrm{ml}$ overnight culture were added and mixed. A 24-deep well plate was filled with $5 \mathrm{ml}$ of this mixture per well and incubated at $32^{\circ} \mathrm{C}$ for 40 minutes on a thermoblock (Eppendorf, USA). Subsequently, the curd was cut with a custom-made sterile stirring device for 20 minutes (stirring for 20 seconds, resting for 3 minutes) followed by 5 minutes of resting. The plate was sealed with an adhesive cover (Microseal $®$, Bio-Rad, USA) and centrifuged at $500 \times g$ for 30 minutes at $30^{\circ} \mathrm{C}$. The whey was removed and replaced by $2 \mathrm{ml}$ sterile water pre-heated at $45^{\circ} \mathrm{C}$. The curd was cut for 40 minutes at $35^{\circ} \mathrm{C}$ and rested for 20 minutes. The plate was sealed again and centrifuged 
for 1 hour at $4800 \times g$ at $30^{\circ} \mathrm{C}$. After all the whey was removed, the plate was sealed with a gas-permeable seal (BREATHseal ${ }^{\mathrm{TM}}$, Greinier Bio One, Germany) and incubated at $30^{\circ} \mathrm{C}$. After overnight incubation, $50 \mu \mathrm{l}$ of a $19 \%(\mathrm{w} / \mathrm{v}) \mathrm{NaCl}$ solution was added to each well followed by centrifugation for 5 minutes at $99 \times g$. The plate was sealed with a gas-permeable seal, placed in a jar under anaerobic conditions and incubated at $12^{\circ} \mathrm{C}$. After $1,2,4$ and 8 weeks, 6 cheeses per time point were sampled for analysis of the volatile organic compounds.

\section{Cell dry weight determination}

The cell dry weight was determined as previously described (van Mastrigt et al., 2018b). Approximately $3 \mathrm{~mL}$ sample was used for each determination.

\section{Cell viability detection}

The viability of cells in the culture was determined by LIVE/DEAD Baclight Bacterial Viability kit (Molecular Probes Europe, The Netherlands). Cells were stained by incubating $100 \mu \mathrm{l}$ culture with $3.34 \mu \mathrm{M}$ green fluorescent SYTO 9 and $20 \mu \mathrm{M}$ red fluorescent propidium iodide for 10 minutes at room temperature in the dark. The number of green and red cells were counted after visualising them with an X-Cite 120Q excitation light source (Excelitas, USA) at 1000 times magnification with a fluorescent microscope equipped with a camera (Olympus, Japan). Selected images for counting had at least 50 cells.

\section{Volatile organic compounds (VOCs) analysis}

One millilitre sample or approximately $0.5 \mathrm{~g}$ cheese was transferred to a $5 \mathrm{ml} \mathrm{GC}$ vial and stored at $-20^{\circ} \mathrm{C}$ until analysis of the VOCs by headspace solid phase microextraction gas chromatography mass spectrometry (HS SPME GC-MS) according to van Mastrigt et al. (2018a). Aroma profiles were analysed with Chromeleon 7.2 software. The ICIS algorithm was used for peak integration and mass spectral profiles were matched with the NIST main library for identification. One peak (in general the higher $\mathrm{m} / \mathrm{z}$ peak per compound) was used per compound for quantification and 1 or 2 peaks were used for confirmation. Compounds were only considered if the area of the quantitative peak was at least 3 times higher than the peak area in the medium in at least one sample and the peak area was higher than 500 count $\times$ min. Methyl esters, butyl esters and 1-butanol were removed from the analysis as their formation was dependent on the presence of methanol and butanol, which were not produced by the bacteria but present in the medium (1-butanol is used as solvent to make Bacto-tryptone). Alkanes were removed because they were not considered as important flavour compounds in cheese. Compounds were considered produced if the peak area was at least 1.5 
times higher than the peak area in the medium and the peak area was higher than 200 count $\times$ min. The aroma profiles of every biological independent culture was analysed once, except for the two retentostat cultures, which were analysed in duplicate.

\section{Results and discussion}

Lactococcus lactis FM03-V1 was grown in retentostat and batch cultures on a chemically defined medium (CDM) to determine the effect of the cultivation method, imposing slow and fast growth respectively, on the formation of aroma compounds. Furthermore, aroma formation was analysed in batch cultures using three different media (CDM, hydrolysed micellar casein isolate (MCI) and full fat milk) and in a milli-cheese model system, which could indicate the origin of the compounds and show which methods best resemble aroma formation during cheese ripening. We chose to use a milli-cheese model system instead of conventionally produced cheese making for several reasons: i) we wanted to use the single non-proteolytic strain $L$. lactis FM03-V1, which required the addition of an amino acid source to the milk; ii) milli-cheeses resemble conventionally produced cheeses very well including their flavour profile (Bachmann et al., 2009); iii) the milli-cheese system allowed for more replicates and different sampling times.

In batch and retentostat cultures (liquid medium) L. lactis was incubated for 2 weeks, while aroma formation in the milli-cheeses was analysed after 1, 2, 4 and 8 weeks of ripening. During the retentostat cultivations, the biomass increased in 2 weeks from 1 to $6 \mathrm{gDW} / \mathrm{kg}$ and the growth rate gradually decreased from $0.05 \mathrm{~h}^{-1}$ to approximately $0.001 \mathrm{~h}^{-1}$ (Suppl. Fig. S5.1), while the viability remained always above $90 \%$ as determined by live/dead staining. In the batch cultivations, the bacteria grew fast in the first day after which the growth rate decreased due to nutrient depletion and/or low $\mathrm{pH}$.

\section{Qualitative comparison of aroma production}

In total 82 aroma compounds were considered produced in the cultivations (in at least 1 sample at least a 3 times increase after fermentation and a peak area greater than 500 count $\times$ min). The produced compounds were qualitatively compared with each other in a heat map showing production of compounds in specific cultures (Fig. 5.1). Biological replicates had highly similar patterns in the heat map showing that aroma formation was reproducible. Furthermore, we found that both the cultivation method (batch cultivation, retentostat cultivation and milli-cheese) and the medium (CDM, MCI, milk) affected the formation of aroma compounds. 


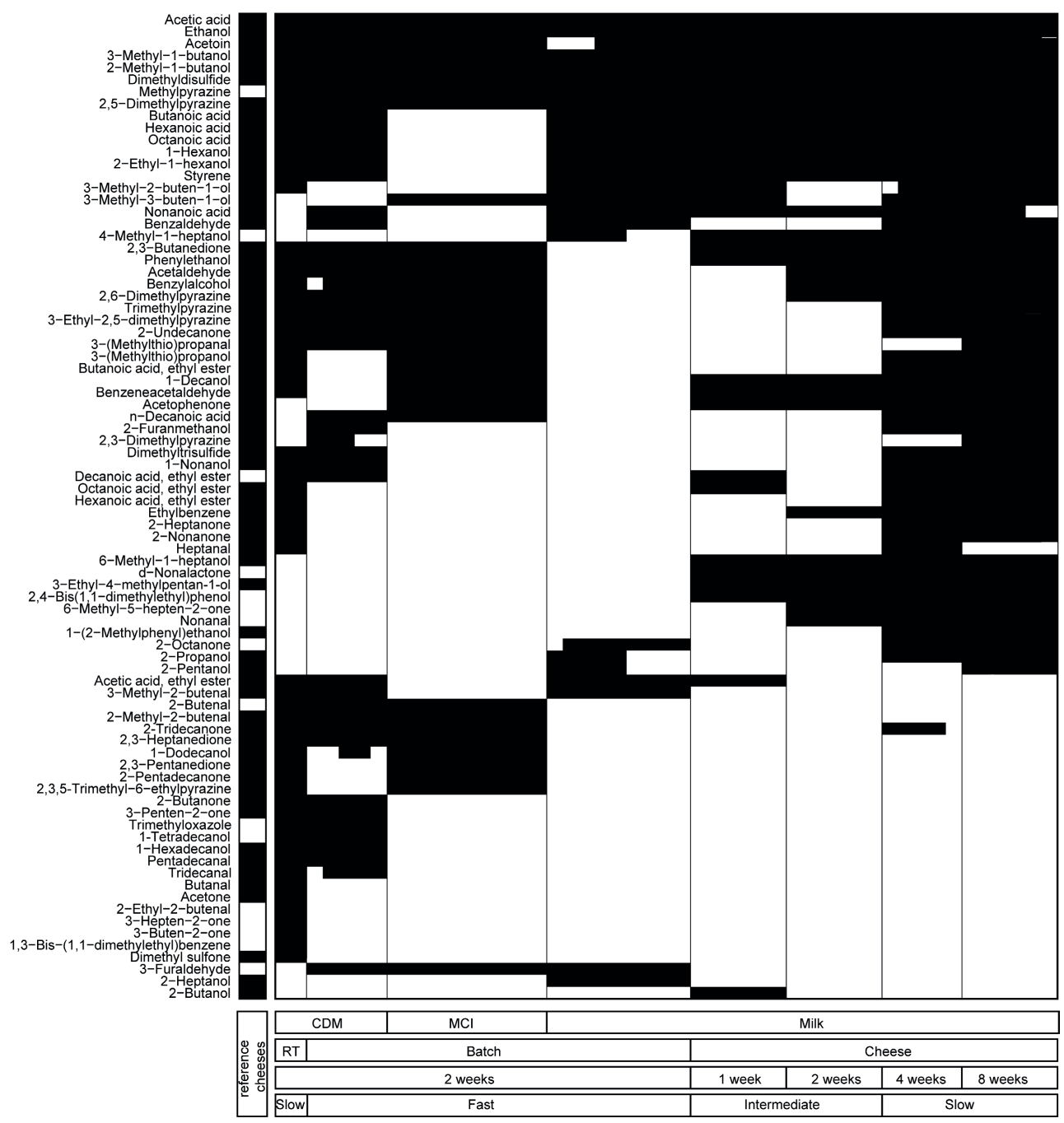

Figure 5.1: Production of VOCs by L. lactis FM03-V1. Colours represent the presence (black) or absence (white) of VOCs after two weeks incubation in batch cultures in chemically defined medium (CDM), hydrolysed micellar casein isolate (MCI), and full fat milk, or in retentostat cultures (RT) in CDM or in a milli-cheese model system after 1, 2, 4 or 8 weeks or ripening. The first column represents compounds that can be found in various cheeses (black: present; (Barbieri et al., 1994, Curioni and Bosset, 2002, Fernández-García et al., 2002, Qian and Reineccius, 2002, Singh et al., 2003, van Leuven et al., 2008)).

Fatty acids were not produced in MCI medium possibly due to the lack of fat in this medium. Interestingly, fatty acids were produced in CDM that also did not contain fat or free fatty acids indicating that free fatty acid were synthesised by the bacteria to end up in volatile aroma compounds. In the batch cultures with 
milk several degradation products of amino acids were not found while being found in batch culture based on CDM and/or MCI, e.g. phenylethanol, benzeneacetylaldehyde, benzylalcohol, acetophenone, 3-(methylthio)propanol, 3(methylthio)propanal and dimethyltrisulfide. In CDM and MCI, amino acids were abundant due to addition of Bacto-tryptone and due to hydrolysis of the proteins, respectively. There were 10 compounds that were lacking in the batch cultures with CDM and milk while being present in the 8-week-old cheeses and retentostat cultures, which suggests that these compounds were only produced by slow growing cells. These compounds include ethyl esters (butanoic acid, ethyl ester, hexanoic acid, ethyl ester and octanoic acid, ethyl ester) and methyl ketones (2heptanone and 2-nonanone). The limiting compound in ethyl ester formation by lactic acid bacteria is considered to be ethanol (Liu et al., 2004). Notably, due to the low growth rates in retentostat cultures, L. lactis produces mainly ethanol, acetate and formate instead of lactate, thereby driving ethyl ester formation. Both esters and methyl ketones strongly contribute to the flavour of the cheese due to their low perception thresholds (Curioni and Bosset, 2002). Esters have fruity notes and are mainly found in Italian type cheese, while methyl ketones are associated with fruity, musty and blue cheese notes and are typically found in mould-ripened cheese.

Thirteen (13) compounds were found only with milk as medium of which 7 compounds were found only in the ripened cheese. These include alcohols (4methyl-1-heptanol, 6-methyl-1-heptanol, 3-ethyl-4-methylpentanol, 2-propanol, 2-butanol, 2-pentanol and 2-heptanol) and ketones (2-octanone, 6-methyl-5hepten-2-one and $\delta$-nonalactone) that most likely originate from degradation of milk fats.

In total 27 compounds were identified that were not found in the 4- or 8-weekold milli-cheeses, of which 24 were found in the retentostat cultures including 17 compounds that can be found in ripened cheese (Barbieri et al., 1994, Curioni and Bosset, 2002, Fernández-García et al., 2002, Qian and Reineccius, 2002, Singh et al., 2003, van Leuven et al., 2008). These 24 compounds compounds include 2 diketones with a buttery, cheesy flavour (2,3-pentanedione and 2,3heptanedione) (Burdock, 2010), 3 unsaturated ketones with a sweet, cheesy flavour (3-buten-2-one, 3-penten-2-one and 3-hepten-2-one) (Pripdeevech et al., 2011), 4 unsaturated short aldehydes with a green fruity aroma (2-butenal, 3methyl-2-butenal, 2-methyl-2-butenal and 2-ethyl-2-butenal) (Luna et al., 2006) and 7 long straight-chain alcohols, ketones and aldehydes that have a relatively high perception threshold and are conceivably less important (1-dodecanol, 1tetradecanol, 1-hexadecanol, 2-tridecanone, 2-pentadecanone, tridecanal and 
pentadecanal). Based on these odour descriptions, production of these compounds is considered positive.

Comparing all produced aroma compounds with ripened milli-cheeses revealed that the aroma profile of the retentostat cultures had the biggest overlap with 8week-old milli-cheeses ( 36 compounds in common) despite the use of a chemically defined medium (Fig. 5.2). Moreover, many of the aroma compounds that were produced in the retentostat cultures but not in the milli-cheeses can be found in other types of cheeses (Barbieri et al., 1994, Curioni and Bosset, 2002, Fernández-García et al., 2002, Qian and Reineccius, 2002, Singh et al., 2003, van Leuven et al., 2008) and/or have an odour description that is considered positive. This shows that retentostat cultivation is an interesting technique to produce aroma compounds typical for cheese.
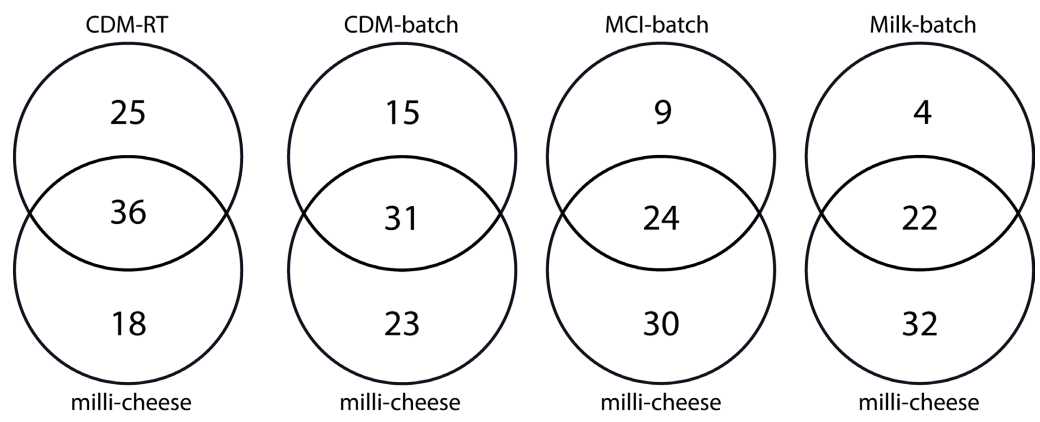

Figure 5.2: Qualitative comparison of the production of VOCs by L. lactis FM03-V1. VOCs production was compared between the liquid cultures and a milli-cheese model system after 8 weeks of ripening.

\section{Quantitative comparison presence of aroma}

To identify compounds that were underrepresented in the retentostat cultures, we quantitatively compared aroma formation in the retentostat cultures with that in the 8-week-old cheese. We combined this information with the putative origin of the compounds indicating which substrates could be added to the medium to enhance flavour formation during retentostat cultivation to better resemble aroma formation during cheese ripening (Fig. 5.3). In total 25 out of 67 compounds found in the 8-week-old milli-cheese model system were found in higher amounts in the retentostat culture and 33 compounds were produced in the retentostat cultures but in lower amounts. Only 9 compounds were lacking in the retentostat cultures (peak area in retentostat culture $<1 \%$ of milli-cheese). 


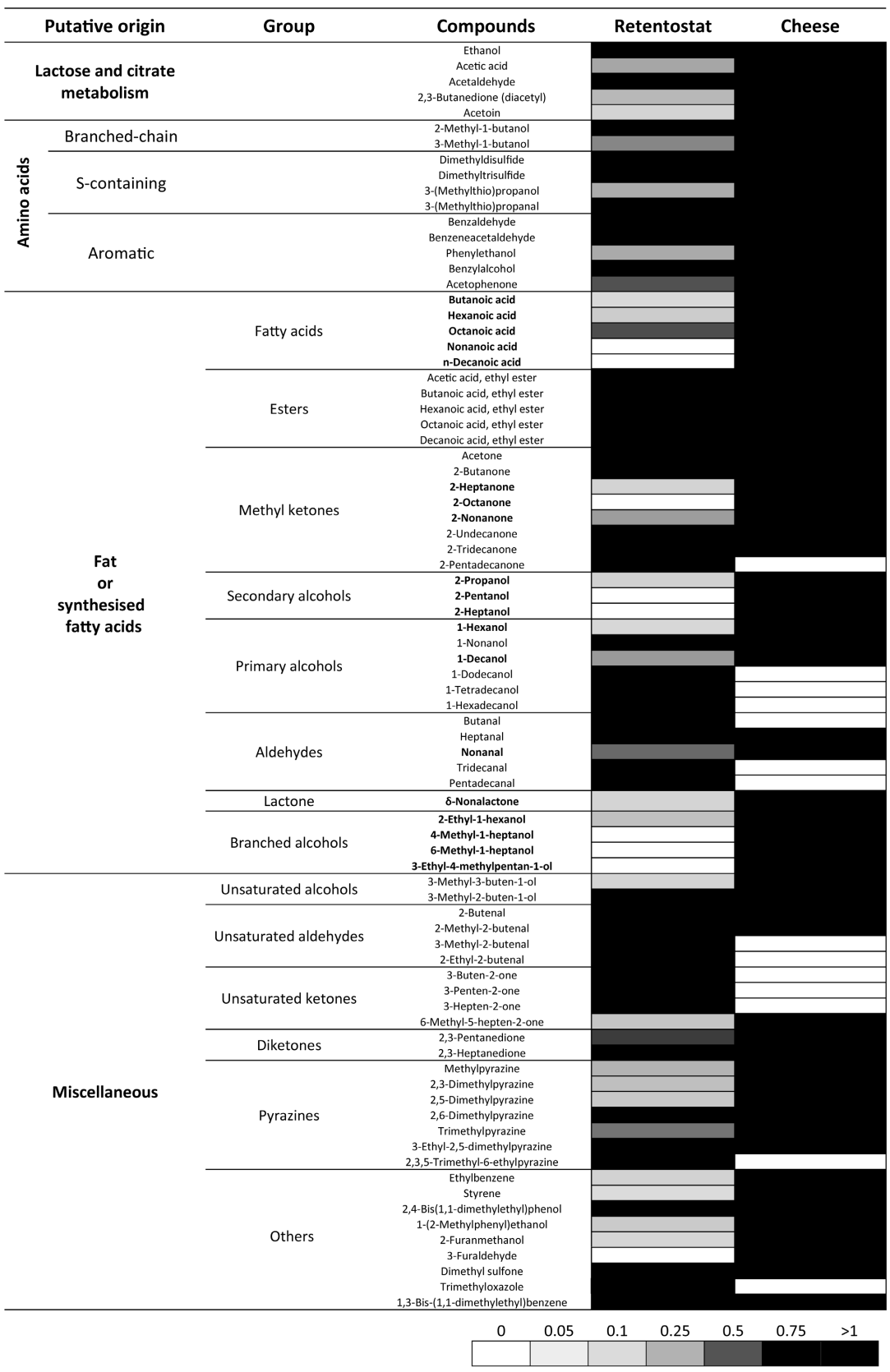

Figure 5.3: Quantitative comparison of VOCs in retentostat cultures of L. lactis FM03-V1 with a milli-cheese model system. Shades of grey correspond to the abundance relative to the 8-week-old milli-cheese (see legend at bottom of table). Black: similar or higher than in milli-cheese; grey: lower than in milli-cheese; white: absent. Production of compounds in bold was lower in the retentostat cultures but might increase by addition of free fatty acids to the medium. 
Groups of compounds that were underrepresented in the retentostat cultures were mainly medium-chain free fatty acids, medium-chain methyl ketones, secondary alcohols, medium-chain primary alcohols and branched-chain primary alcohols (Fig. 5.3). These compounds most likely originate from the milk fats via lipolysis and subsequent conversion of the released free fatty acids to either methyl ketones and secondary alcohols via $\beta$-oxidation or primary alcohols and branchedchain alcohols via reduction of the free fatty acids. Fat or free fatty acids were not present in the chemically defined medium and therefore the fatty acids that were found were synthesised by the bacteria. Fatty acids biosynthesis involves the addition of two carbon units in each cycle explaining why only even numbered fatty acids were found in the retentostat cultures. During $\beta$-oxidation, fatty acids are first oxidised to a-keto acids, which are further decarboxylated to their corresponding methyl ketones with one carbon atom less, such as 2-heptanone, 2-nonanone and 2-undecanone. Ultimately, the methyl ketones can be reduced to secondary alcohols (Collins et al., 2003). Fatty acid biosynthesis in bacteria is regulated by product inhibition by long-chain acyl-ACPs (Jiang and Cronan, 1994) preventing high levels of fatty acid production. Therefore, it will be hard to find conditions to stimulate fatty acid production and it is more convenient to add fat or a mixture of free fatty acids to improve the production of fatty acid-related aroma compounds. Moreover, this enables the production of even numbered methyl ketones that are currently lacking in the retentostat cultures. Because fat will most likely impair the retentostat cultivation by clogging the filter, free fatty acids are preferred. Care should be taken with the addition of free fatty acids as high levels can lead to rancidity and unbalanced flavours (Collins et al., 2003). As fatty acids and their derived compounds were also lacking after fermentation of MCI (Suppl. Fig. S5.2), addition of free fatty acids could also improve flavour formation in MCI.

\section{Conclusions}

Aroma production by L. lactis was clearly affected by the type of medium (CDM, MCI or milk) and the cultivation method (batch, retentostat or milli-cheese). Aroma formation in a milli-cheese model system was best resembled using retentostat cultivation in which the bacteria hardly grew mimicking cheese ripening conditions. Retentostat cultivation resulted in the highest number of known cheese aroma compounds (52) and an increase in esters, methyl ketones, diketones and unsaturated ketones, which are important cheese flavours and/or have cheese-like or buttery flavours. In the retentostat cultures as well as in batch cultures with CDM and MCI, free fatty acids and their corresponding degradation products (methyl ketones, secondary alcohols and primary alcohols) were 
underrepresented compared to the milli-cheese. Addition of a mixture of free fatty acids to CDM and MCI could help to enhance flavour formation in these media to better resemble flavour formation in cheese. This study shows that typical cheese aroma compounds can be produced by lactic acid bacteria outside the cheese matrix, which offers opportunities for future applications as food supplements in dairy or non-dairy products.

\section{Acknowledgement}

This work was financially supported by Arla Foods (Aarhus, Denmark). 


\section{Supplementary materials}

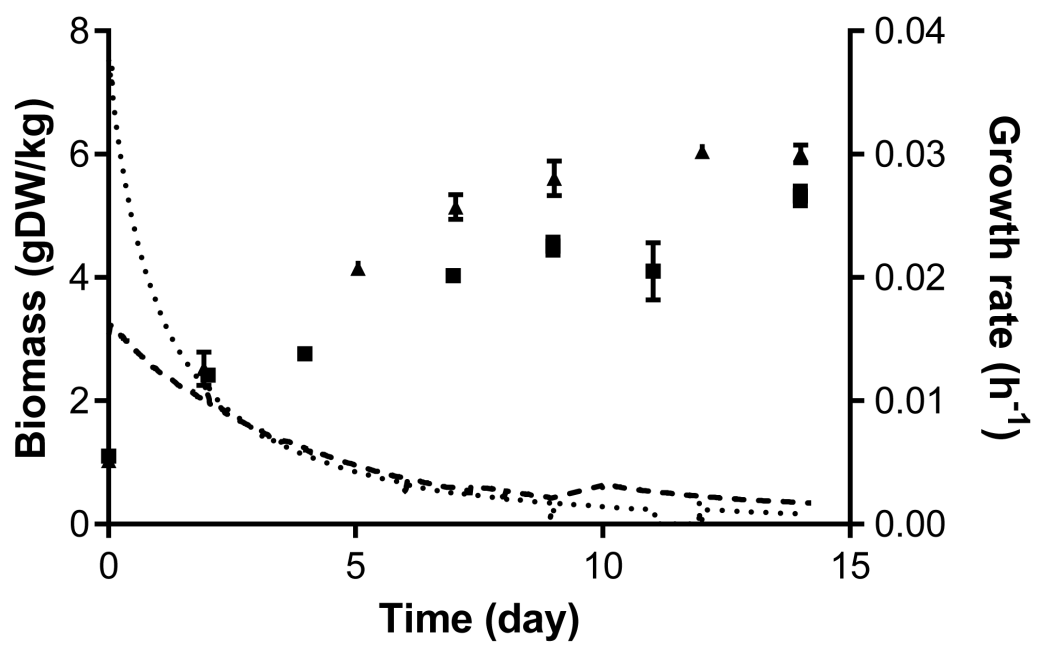

Supplementary Figure S5.1: Biomass accumulation of L. lactis FM03-V1 in two independent retentostat cultures at a dilution rate of $0.05 \mathrm{~h}^{-1}$ and the corresponding growth rates. Squares and triangles represent biomass concentrations of retentostat 1 and 2, respectively. Dashed and dotted lines represent the estimated growth rates in retentostat culture 1 and 2, respectively. Error bars represent the standard deviation of technical duplicates. 


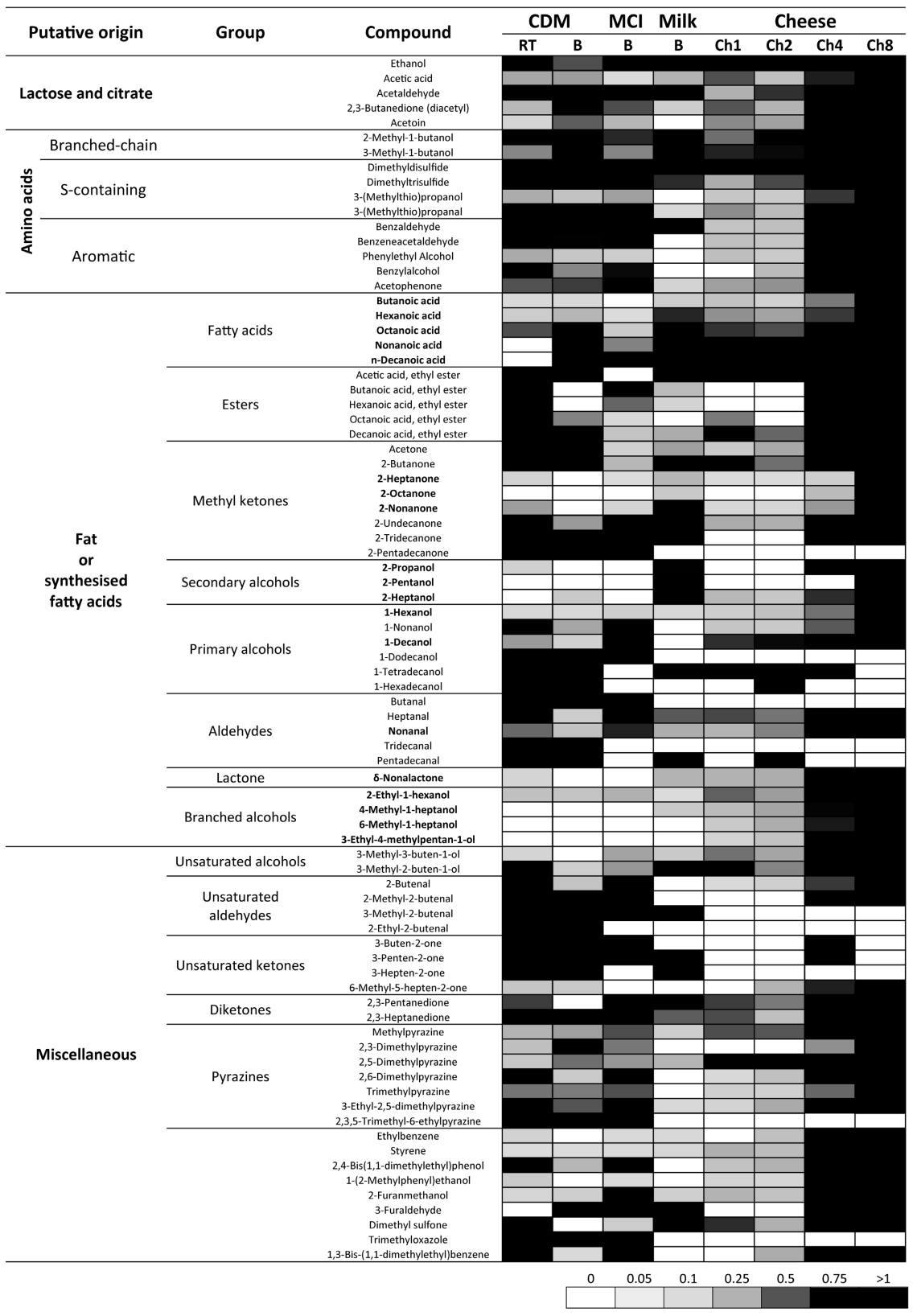

Supplementary Figure S5.2: Quantitative comparison of the volatile organic compounds in liquid cultures of $L$. lactis FM03-V1 and in a milli-cheese model system after 1, 2, 4 and 8 weeks of ripening. Shades of grey correspond to the abundance relative to the 8 -week-old milli-cheese (see legend at bottom of table). Black: similar or higher than milli-cheese; grey: lower than in milli-cheese; white: absent. B: batch cultivation; RT: retentostat cultivation, CDM: chemically defined medium; MCI: hydrolysed micellar casein isolate medium. 


\section{References}

Azarnia, S., Robert, N. and Lee, B. (2006). Biotechnological methods to accelerate Cheddar cheese ripening. Crit. Rev. Biotechnol. 26(3): 121-143.

Bachmann, H., Kruijswijk, Z., Molenaar, D., Kleerebezem, M. and van Hylckama Vlieg, J. E. T. (2009). A high-throughput cheese manufacturing model for effective cheese starter culture screening. J. Dairy Sci. 92(12): 5868-5882.

Barbieri, G., Bolzoni, L., Careri, M., Mangia, A., Parolari, G., Spagnoli, S. and Virgili, R. (1994). Study of the volatile fraction of Parmesan cheese. J. Agric. Food Chem. 42(5): $1170-1176$.

Burdock, G. A. (2010). Fenaroli's handbook of flavor ingredients (6th ed.). Boca Raton, USA: CRC Press.

Collins, Y. F., McSweeney, P. L. H. and Wilkinson, M. G. (2003). Lipolysis and free fatty acid catabolism in cheese: a review of current knowledge. Int. Dairy J. 13(11): 841866.

Curioni, P. M. G. and Bosset, J. O. (2002). Key odorants in various cheese types as determined by gas chromatography-olfactometry. Int. Dairy J. 12(12): 959-984.

Ercan, O., Bisschops, M. M. M., Overkamp, W., Jorgensen, T. R., Ram, A. F., Smid, E. J., Pronk, J. T., Kuipers, O. P., Daran-Lapujade, P. and Kleerebezem, M. (2015). Physiological and transcriptional responses of different industrial microbes at nearzero specific growth rates. Appl. Environ. Microbiol. 81(17): 5662-5670.

Fernández-García, E., Serrano, C. and Nuñez, M. (2002). Volatile fraction and sensory characteristics of Manchego cheese. 2. Seasonal variation. J. Dairy Res. 69(4): 595604.

Fox, P. F., Guinee, T. P., Cogan, T. M. and McSweeney, P. L. H. (2017). Biochemistry of cheese ripening. In Fox, P. F., Guinee, T. P., Cogan, T. M. and McSweeney, P. L. H. (Eds.), Fundamentals of cheese science (pp. 391-442). Boston, MA: Springer.

Jiang, P. and Cronan, J. E. (1994). Inhibition of fatty acid synthesis in Escherichia coli in the absence of phospholipid synthesis and release of inhibition by thioesterase action. J. Bacteriol. 176(10): 2814-2821.

Liu, S.-Q., Holland, R. and Crow, V. L. (2004). Esters and their biosynthesis in fermented dairy products: a review. Int. Dairy J. 14(11): 923-945.

Luna, G., Morales, M. T. and Aparicio, R. (2006). Characterisation of 39 varietal virgin olive oils by their volatile compositions. Food Chem. 98(2): 243-252.

McSweeney, P. L. H. (2004). Biochemistry of cheese ripening. Int. J. Dairy Technol. 57(23): $127-144$.

Pripdeevech, P., Khummueng, W. and Park, S.-K. (2011). Identification of odor-active components of agarwood essential oils from Thailand by solid phase microextraction-GC/MS and GC-O. J. Essent. Oil Res. 23(4): 46-53.

Qian, M. and Reineccius, G. (2002). Identification of aroma compounds in ParmigianoReggiano cheese by gas chromatography/olfactometry. J. Dairy Sci. 85(6): 13621369.

Singh, T. K., Drake, M. A. and Cadwallader, K. R. (2003). Flavor of Cheddar cheese: a chemical and sensory perspective. Compr. Rev. Food Sci. Food Saf. 2(4): 166-189.

Smid, E. J. and Kleerebezem, M. (2014). Production of aroma compounds in lactic fermentations. Annu. Rev. Food Sci. Technol. 5(1): 313-326.

Spus, M., Liu, H., Wels, M., Abee, T. and Smid, E. J. (2017). Isolation and characterization of Lactobacillus helveticus DSM 20075 variants with improved autolytic capacity. Int. J. Food Microbiol. 241(Supplement C): 173-180.

van de Bunt, B., Bron, P. A., Sijtsma, L., de Vos, W. M. and Hugenholtz, J. (2014). Use of non-growing Lactococcus lactis cell suspensions for production of volatile metabolites with direct relevance for flavour formation during dairy fermentations. Microb. Cell Fact. 13(1): 176.

van Leuven, I., van Caelenberg, T. and Dirinck, P. (2008). Aroma characterisation of Goudatype cheeses. Int. Dairy J. 18(8): 790-800. 
van Mastrigt, O., Abee, T. and Smid, E. J. (2017). Complete genome sequences of Lactococcus lactis subsp. lactis bv. diacetylactis FM03 and Leuconostoc mesenteroides FM06 isolated from cheese. Genome Announc. 5(28): e00633-17.

van Mastrigt, O., Abee, T., Lillevang, S. K. and Smid, E. J. (2018a). Quantitative physiology and aroma formation of a dairy Lactococcus lactis at near-zero growth rates. Food Microbiol. 73: 216-226.

van Mastrigt, O., Mager, E. E., Jamin, C., Abee, T. and Smid, E. J. (2018b). Citrate, low pH and amino acid limitation induce citrate utilisation in Lactococcus lactis biovar diacetylactis. Microb. Biotechnol. 11(2): 369-380. 



\section{Chapter 6}

\section{Quantitative physiology and aroma formation of a dairy Lactococcus lactis at near-zero growth rates}

Oscar van Mastrigt, Tjakko Abee, Søren K. Lillevang \& Eddy J. Smid

Published in Food Microbiology, August 2018, 73:216-226 Published online: 3 Feb 2018, doi: 10.1016/j.fm.2018.01.027 


\begin{abstract}
During food fermentation processes like cheese ripening, lactic acid bacteria (LAB) encounter long periods of nutrient limitation leading to slow growth. Particular $L A B$ survive these periods while still contributing to flavour formation in the fermented product. In this study the dairy Lactococcus lactis biovar diacetylactis FM03-V1 is grown in retentostat cultures to study its physiology and aroma formation capacity at near-zero growth rates. During the cultivations, the growth rate decreased from $0.025 \mathrm{~h}^{-1}$ to less than $0.001 \mathrm{~h}^{-1}$ in 37 days, while the viability remained above $80 \%$. The maintenance coefficient of this dairy strain decreased by a factor 7 at near-zero growth rates compared to high growth rates (from $2.43 \pm 0.35$ to $0.36 \pm 0.03 \mathrm{mmol}$ ATP.gDW $\left.\mathrm{gW}^{-1} \cdot \mathrm{h}^{-1}\right)$. In the retentostat cultures, 62 different volatile organic compounds were identified by HS SPME GC-MS. Changes in the aroma profile resembled some of the biochemical changes occurring during cheese ripening and reflected amino acid catabolism, metabolism of fatty acids and conversion of acetoin into 2-butanone. Analysis of complete and cell-free samples of the retentostat cultures showed that particular lipophilic compounds, mainly long-chain alcohols, aldehydes and esters, accumulated in the cells, most likely in the cell membranes. In conclusion, retentostat cultivation offers a unique tool to study aroma formation by lactic acid bacteria under industrially relevant growth conditions.
\end{abstract}




\section{Introduction}

In natural environments, microorganisms generally live in a feast or famine existence due to variable availabilities of nutrient and energy sources (Koch, 1971, Morita, 1993) and it has been suggested that energy limitation is the prevailing physiological state among microorganisms on Earth (Brock, 1971, Morita, 1997). The physiological state of these organisms is poorly represented in laboratory experiments with nutrient-rich batch cultures, in which metabolic rates are several orders of magnitude higher than in oligotrophic environments (Hoehler and Jorgensen, 2013). Also during particular industrial fermentations bacteria may experience strongly restricted access to nutrients for long periods of time. For instance, during cheese ripening lactic acid bacteria (LAB) encounter such conditions (Martley and Crow, 1993, McSweeney and Fox, 2004, Thomas and Pearce, 1981). This leads to severe reduction of the growth rate. Particular LAB survive these periods of extremely slow growth, while still contributing to aroma formation in the fermented product (Erkus et al., 2013).

Conditions of strong nutrient restriction and extremely slow growth could be mimicked in the laboratory using retentostat cultivation (Boender et al., 2009). This unique fermentation technique is a modification of chemostat cultivation in which culture effluent is removed, but biomass is retained by a filter in the effluent line. Because nutrients are continuously fed to the reactor while biomass is retained, biomass will accumulate leading to a gradual decrease in the substrate availability per cell. Metabolic energy is distributed to either growth or non-growth (maintenance) processes (Pirt, 1965). When the maintenance energy coefficient is assumed to be growth rate independent as proposed by Pirt (Pirt, 1965), the decreasing substrate availability leads to growth rates approaching zero. Finally, all metabolic energy that can be derived from the substrate will be used for maintenance processes.

Retentostat cultivation has been used for studying the physiology of some industrially relevant microorganisms, like Saccharomyces cerevisiae, Bacillus subtilis, Lactobacillus plantarum and Lactococcus lactis (Boender et al., 2009, Ercan et al., 2013, Ercan et al., 2015a, Goffin et al., 2010, Overkamp et al., 2015). Doubling times increased till one year and the viability remained high. Different adaptation strategies were observed, like alternative carbon-source utilisation, upregulation of stress response genes and increased robustness (Boender et al., 2011, Ercan et al., 2015a, Ercan et al., 2015b, Vos et al., 2016). However, the effect of extremely slow growth on aroma formation has never been studied using retentostat cultivation. Moreover, the study with $L$. lactis was performed with the plant-associated L. lactis subsp. lactis KF147, while the main aroma producers of 
this species during cheese ripening are strains of L. lactis subsp. lactis biovar diacetylactis (Erkus et al., 2013). This biovariety is able to consume citrate, which can be converted into the buttery aromas diacetyl and acetoin (Harvey and Collins, 1961).

Besides these buttery aromas, various other categories of compounds are responsible for aroma in fermented foods, such as alcohols, aldehydes, ketones, fatty acids, esters and sulphur compounds (Engels et al., 1997). Precursors for these compounds originate from all main components in food materials (i.e. proteins, lipid and carbohydrates) (Smit et al., 2005b). Conversion of most precursors into aroma compounds relies on functional metabolic pathways (Smid and Kleerebezem, 2014) and microorganisms might activate certain pathways in response to specific conditions.

In this study, a dairy strain of Lactococcus lactis subsp. lactis biovar diacetylactis was grown in retentostat cultures to study the effect of near-zero growth rates on the physiology and aroma formation capacity of this bacterium.

\section{Materials and methods}

\section{Strain and media}

In this study Lactococcus lactis subsp. lactis biovar diacetylactis FM03-V1 has been used, which has been isolated from 10-week-old Sams $\emptyset$ cheese (van Mastrigt et al., 2018). For chemostat and retentostat cultivations, the bacteria were streaked from a $-80^{\circ} \mathrm{C}$ stock on M17 agar plates supplemented with $0.5 \%(\mathrm{w} / \mathrm{v})$ lactose (LM17). After incubation at $30^{\circ} \mathrm{C}$ for 2 days, a single colony was inoculated in $10 \mathrm{~mL}$ lactose-limited chemically defined medium (van Mastrigt et al., 2018) and grown overnight at $30^{\circ} \mathrm{C}$. This overnight culture was used to inoculate the bioreactor at $1 \%(\mathrm{v} / \mathrm{v})$ inoculation level. The chemically defined medium that was used for all retentostat cultivations contained as main carbon and nitrogen sources $0.5 \%(\mathrm{w} / \mathrm{w})$ lactose, $10 \mathrm{mM}\left(\mathrm{NH}_{4}\right)_{3}$ citrate and 1\% (w/w) Bacto-Tryptone and was prepared in $20 \mathrm{~L}$ batches. For chemostat cultivations, the media contained either 0 or $10 \mathrm{mM}\left(\mathrm{NH}_{4}\right)_{3}$ citrate.

\section{Chemostat cultivation}

Bacteria were grown at dilution rates between 0.05 and $0.4 \mathrm{~h}^{-1}$ in bioreactors with a working volume of $0.5 \mathrm{~L}$ (Multifors, Infors $\mathrm{HT}$, Switzerland). The stirring speed was set at $300 \mathrm{rpm}$, the temperature was kept constant at $30^{\circ} \mathrm{C}$ and the $\mathrm{pH}$ was controlled at 5.5 by automatic addition of $5 \mathrm{M} \mathrm{NaOH}$. The headspace was flushed 
with nitrogen gas at a rate of $0.1 \mathrm{~L} / \mathrm{min}$ to maintain anaerobic conditions. The optical density at $600 \mathrm{~nm}$ was continuously measured using an internal probe (Trucell2, Finesse, USA). Samples were taken after reaching steady state conditions, which were considered to be achieved after a minimum of five volume changes at which the optical density remained constant. The dilution rate was changed by controlling the feed rate, which was monitored by continuously weighing the medium vessel.

\section{Retentostat cultivation}

Independent retentostat cultivations were performed in bioreactors with a working volume of $1 \mathrm{~L}$ (Multifors, Infors HT, Switzerland). All conditions were the same as in the chemostat cultivation except that a stirring speed of $400 \mathrm{rpm}$ was used. After a steady state was achieved in a chemostat cultivation at a dilution rate of $0.025 \mathrm{~h}^{-1}$, retentostat cultivation was initiated by removing the effluent via a sterilisable polyethersulfone cross-flow filter (Spectrum laboratories, USA). This filter was connected via an outer loop. Residence times of biomass in the outer loop were minimised. As removal of samples could interfere with biomass accumulation, sample volumes and frequency were minimised to maximum $10 \mathrm{ml}$ every 3 to 4 days.

\section{Cell dry weight determination}

The cell dry weight was determined as described by van Mastrigt et al. (2018). Briefly, 2 and $5 \mathrm{ml}$ sample was passed through a pre-weighted $0.2 \mu \mathrm{m}$ membrane filter (Pall Corporation, USA) by a vacuum filtration unit. Subsequently, the filter was dried at $80^{\circ} \mathrm{C}$ for 48 hours and weighted again on an analytical balance.

\section{Analysis of extracellular metabolites}

Lactose, citrate, lactate, acetate, ethanol, formate, pyruvate and acetoin were quantified by High Performance Liquid Chromatography (HPLC) as described by van Mastrigt et al. ( 2018).

\section{Volatile organic compounds analysis}

VOC detection by HS SPME GC-MS

To determine the volatile organic compounds (VOCs), $100 \mu \mathrm{l}$ sample was transferred to a $5 \mathrm{ml} \mathrm{GC}$ vial. Samples were kept frozen $\left(-20^{\circ} \mathrm{C}\right)$ until analysis by headspace solid phase microextraction gas chromatography mass spectrometry 
(HS SPME GC-MS) (van Rijswijck et al., 2017). Samples were defrosted and incubated for 5 minutes at $60^{\circ} \mathrm{C}$ with agitation. Subsequently, volatile organic compounds were extracted from the samples for 20 minutes at $60^{\circ} \mathrm{C}$ using a Solid Phase Microextraction fiber ( $85 \mathrm{~mm}$ CAR/PDMS, Supelco, USA). The compounds were desorbed from the fiber for 10 minutes on a Stabilwax ${ }^{\circledR}$-DA-Crossband ${ }^{\circledR}$ Carbowax ${ }^{\circledR}$-polyethylene-glycol column (30 m length, $0.25 \mathrm{mmID}, 0.5 \mu \mathrm{m} \mathrm{df}$ ). The settings on the gas chromatograph were PTV Split-less mode 5 minutes at $250^{\circ} \mathrm{C}$. Helium was used as carrier gas at a constant flow of $1.5 \mathrm{ml} / \mathrm{min}$. The temperature of the $\mathrm{GC}$ oven was initially $40^{\circ} \mathrm{C}$. After 2 minutes, the temperature was raised to $240^{\circ} \mathrm{C}$ at a rate of $10^{\circ} \mathrm{C} / \mathrm{min}$ and kept at $240^{\circ} \mathrm{C}$ for 5 minutes. Mass spectral data was collected over a range of $\mathrm{m} / \mathrm{z} 33-250$ in full scan mode with 3.0030 scans/second.

\section{VOC identification and quantification}

VOC profiles were analysed with Chromeleon 7.2 software. The ICIS algorithm was used for peak integration and the NIST main library was used for identification by matching mass spectral profiles with the profiles in NIST. One quantifying peak (in general the highest $\mathrm{m} / \mathrm{z}$ peak per compound) was used per compound for quantification, while 1 or 2 confirming peaks were used for confirmation.

\section{VOC statistics}

Every sample was analysed in duplicate and the average was taken of these technical replicates. To identify if compounds were produced by the bacteria, we used the criteria that the quantifying peak area in the retentostat samples had to be higher than the quantifying peak area in the medium. To determine if production of the volatile organic compounds changed in time, linear mixedeffects modelling was used to correct for the nested structure of the data: samples were taken at different times from the same cultivation. Therefore, time nested within cultivation was used as random factor. A linear mixed effect model (LME) with time as explanatory variable was compared with a generalised least-squares model (GLS) by restricted maximum likelihood estimation (REML). A compound was considered to change significantly in time if the $P$ value for the fixed factor time was lower than 0.00094 (Bonferroni correction; $p<0.05 / 53$ )

To analyse if compounds were located in the cells, the volatile organic compounds of complete samples and cell-free samples were quantified. Subsequently, paired t-tests were performed for each compound to determine if compounds were significantly more abundant in complete samples. To correct for the large number of compounds, Bonferroni correction was used $(P$ value $<0.05 / 61)$ 


\section{$\log P$}

The octanol-water partitioning coefficient (log $\mathrm{P}_{\text {oct} / \text { wat }}$ ) was either taken from literature (Burkhard et al., 1985, Hansch et al., 1995, Sangster, 1989, Tewari et al., 1982, Valvani et al., 1981, Yamagami et al., 1991) or if not found in literature it was predicted by EPIWEB 4.1 software.

\section{Cell viability}

The viability of cells in the culture was determined by LIVE/DEAD Baclight Bacterial Viability kit (Molecular Probes Europe, The Netherlands). To stain the bacteria, $100 \mu \mathrm{l}$ culture was incubated with $3.34 \mu \mathrm{M}$ green fluorescent SYTO 9 and $20 \mu \mathrm{M}$ red fluorescent propidium iodide for 10 minutes at room temperature in the dark. A fluorescent microscope was used for visualisation and estimation of the number of green and red cells. In addition, appropriate dilutions of the culture in peptone physiological salt solution were plated in duplicate on LM17 plates for quantification of colony forming units (CFUs).

\section{Scanning electron microscopy}

The morphology of the cells was analysed by scanning electron microscopy (SEM). Samples were centrifuged $(17000 \times g$ for 1 minute), washed with peptone physiological salt solution (PPS) and resuspended in PPS. A droplet of the suspension was placed onto poly-L-lysine coated coverslips (Corning BioCoat, USA) and allowed to stand for 1 hour at room temperature. After rinsing in phosphate buffered saline (PBS), the samples were fixed in $3 \%$ glutaraldehyde in buffer for 1 hour. This was followed by further rinsing and post-fixing in $1 \%$ osmium tetroxide in buffer for 1 hour. Subsequently, the samples were dehydrated in a graded series of ethanol followed by critical point drying with $\mathrm{CO}_{2}$ (Leica EM CPD300, Leica Microsystems, Germany). The coverslips were fitted onto sample stubs using carbon adhesive tabs and sputter coated with $10 \mathrm{~nm}$ tungsten (Leica SCD500). Samples were imaged at $2 \mathrm{KV}, 6 \mathrm{pA}$, at room temperature in a field emission scanning electron microscope (Magellan 400, FEI Company, USA).

\section{Substrate utilisation capacity}

The lactose and citrate utilisation capacity were determined by monitoring the decrease in lactose and citrate in time in cell suspension as previously described (van Mastrigt et al., 2018). Briefly, $10 \mathrm{ml}$ cell suspension of exponentially or retentostat-grown cells was centrifuged at $6000 \times g$ for 5 minutes. The pellets were washed with physiological salt solution and resuspended in chemically defined 
medium of $\mathrm{pH} 5.5$ and incubated at $30^{\circ} \mathrm{C}$. This medium was equal to the chemically defined medium used for the retentostat cultivation except that acetate was omitted. Samples were taken at regular intervals followed by direct centrifugation $\left(17000 \times g\right.$ for 1 minute at $\left.4^{\circ} \mathrm{C}\right)$. The supernatant was stored at $-20^{\circ} \mathrm{C}$ until determination of the lactose and citrate concentration by HPLC. The lactose and citrate utilisation capacity was calculated by dividing the lactose and citrate utilisation rate by the dry weight concentration, which was determined just before the first cell harvesting step.

\section{Protein content}

The protein content was determined by cell lysis using bead-beating followed by protein determination using the Pierce ${ }^{\mathrm{TM}}$ Coomassie Plus (Bradford) assay kit (Thermo Scientific, USA). Cells were either harvested at the end of retentostat cultures or from batch cultures in the exponential phase. For the batch cultures, L. lactis FM03-V1 was streaked onto a LM17 plate and incubated for 3 days at $30^{\circ} \mathrm{C}$. A single colony was inoculated in chemically defined medium with lactose and citrate as main carbon sources and grown overnight at $30^{\circ} \mathrm{C}$. One $\mathrm{ml}$ was transferred to $14 \mathrm{ml}$ fresh medium and incubated for 3 hours at $30^{\circ} \mathrm{C}$. When the optical density at $600 \mathrm{~nm}$ reached $0.5-0.6,5 \mathrm{ml}$ was used to determine the cell dry weight according to the previous described protocol. The remaining sample was centrifuged $(6000 \times g, 5$ minutes) and washed three times with $1 \mathrm{ml} 0.85 \%$ $\mathrm{NaCl}$. The sample was resuspended in $1 \mathrm{ml}$ demineralised water and transferred to a lysis matrix tube (Matrix B, $0.1 \mathrm{~mm}$ silica spheres, Bio-Rad). The suspension was bead-beated in six cycles of 30 seconds beating $(6.0 \mathrm{~m} / \mathrm{s}$; Bio-Rad Fastprep$24)$ and 5 minutes cooling on ice. Subsequently, tubes were centrifuged $(17000 \times g$ for 20 seconds) and the supernatant was transferred to a new tube and used for the protein determination.

For the retentostat cultivations, cells were harvested at the end of the cultivation (37 days). The biomass concentration was determined as described before. Due to the high biomass concentration only $0.5 \mathrm{~mL}$ sample was used. The cells were washed three times with $1 \mathrm{ml} 0.85 \% \mathrm{NaCl}$ and resuspended in $1 \mathrm{ml}$ demineralised water. The rest of the protocol is the same as for the batch cultures described before.

The protein concentration in the suspensions was determined with the Pierce ${ }^{\mathrm{TM}}$ Coomassie Plus (Bradford) assay kit (Thermo Scientific, USA) according to the manufacturer's procedure. Bovine serum albumin (BSA) was used as standard. Both standards and samples were analysed in triplicate. The protein content was 
determined for three independent batch cultures and two independent retentostat cultures were analysed in triplicate.

\section{Modelling biomass accumulation for estimation maintenance}

The accumulation of biomass was modelled using a modified Verseveld equation (van Verseveld et al., 1986), in which the metabolic changes resulting in a different ATP yield on substrate were taken into account:

$$
C_{X}(t)=\left(C_{X, 0}-\frac{D \cdot\left(C_{S, i n}-C_{S}\right) \cdot Y_{A T P / S}}{m_{A T P}}\right) \cdot e^{-m_{A T P} \cdot Y_{x / A T P}^{\max } \cdot t}+\frac{D \cdot\left(C_{S, i n}-C_{S}\right) \cdot Y_{A T P / S}}{m_{A T P}}
$$

in which $\mathrm{C}_{\mathrm{x}}$ is the biomass concentration $(\mathrm{gDW} / \mathrm{kg}), \mathrm{C}_{\mathrm{x}, 0}$ is the initial biomass concentration $(\mathrm{gDW} / \mathrm{kg}), \mathrm{D}$ is the dilution rate $\left(\mathrm{h}^{-1}\right), \mathrm{C}_{\mathrm{s}, \mathrm{in}}$ is the substrate concentration in the medium $\left(14.6 \mathrm{mmol} / \mathrm{kg}\right.$ lactose and $10 \mathrm{mmol} / \mathrm{kg}$ citrate), $\mathrm{C}_{\mathrm{s}}$ is the substrate concentration in the effluent, $\mathrm{Y}_{\mathrm{ATP} / \mathrm{S}}$ is the ATP yield on substrate (mol ATP/CmolS), $m_{\text {ATP }}$ is the maintenance coefficient (mol ATP.gDW ${ }^{-1} \cdot \mathrm{h}^{-1}$ ), $\mathrm{YX}_{\mathrm{X} / \mathrm{ATP}}{ }^{\max }$ is the maximum biomass yield on ATP ( $\mathrm{gDW} / \mathrm{mol}$ ATP) and $\mathrm{t}$ is the time (h).

The ATP yield on substrate was calculated based on the measured metabolite production rates using equation 6.2:

$$
Y_{A T P / S}=\frac{R_{\text {lactate }}+R_{\text {ethanol }}+R_{\text {pyruvate }}+2 \cdot R_{\text {acetoin }}+2 \cdot R_{\text {acetate }}+2 \cdot R_{\text {citrate }}}{-\left(12 \cdot R_{\text {lactose }}+6 \cdot R_{\text {citrate }}\right)}
$$

in which $R_{i}$ is the production rate $(\mathrm{mol} / \mathrm{h}$ ) of compound $\mathrm{i}$. Based on chemostat cultivations with and without citrate, it was assumed that uptake of $1 \mathrm{~mol}$ citrate and its conversion to pyruvate generated 1 mol ATP.

Input data for the modelling were the online optical density measurements, which were first converted into biomass dry weight concentrations using a second-order polynomial relation (Suppl. Fig. S6.1). For fitting the data to equation 6.1, two different models were used: i) both $Y_{X / A T P} \max$ and $m_{\text {ATP }}$ were constant and fitted and ii) $Y_{X / A T P}{ }^{\max }$ was estimated from chemostat cultivations between 0.05 and $0.4 \mathrm{~h}^{-1}$ and the $\mathrm{m}_{\text {ATP }}$ was linearly dependent on the biomass specific ATP production rate $\left(q_{A T P}\right)$ with a maximum $m_{A T P} \max$ (equation 6.3).

$$
m_{A T P}=a+b \cdot q_{A T P} \text { with } m_{a t p} \leq m_{A T P}^{\max }
$$

For this model, $a, b$ and $m_{A T P}{ }^{\max }$ were fitted. Because $q_{A T P}$ and the growth rate are related to each other, both models are equivalent when $m_{\text {ATP }} \leq m_{\text {ATP }}$ max (Supplement A), although the interpretation is different. The second model represents the situation that the maintenance coefficient changes at very low metabolic activities and thus at very low growth rates, while the first model would assume a different maximum biomass yield in chemostat and retentostat cultures. 
The variable parameters were optimised by minimising the sum of squared errors between the model and the data. This was done using the solver add-in of Excel.

\section{Results}

\section{Biomass accumulation, viability and cell morphology}

Lactococcus lactis subsp. lactis biovar diacetylactis FM03-V1 was grown anaerobically in retentostat cultivations on a chemically defined medium containing both lactose and citrate. Two independent cultivations of 37 days were performed to investigate physiological adaptations of this dairy strain towards near-zero growth rates (retentostat 1 and 2). During the cultivations the biomass concentration and the corresponding growth rates were determined every 3 to 4 days (Fig. 6.1).

In both cultures the biomass concentration continuously increased and the growth rate gradually decreased till less than $0.001 \mathrm{~h}^{-1}$ at the end of the cultivation. This corresponds to a doubling time of more than one month. The culture viability was determined using SYTO 9 and propidium iodide (PI) as fluorescent markers combined with fluorescent microscopy. The population was divided into two subpopulations based on the staining characteristics: i) strong green and weak red fluorescence was considered to represent live cells with intact cell membranes, ii) strong red fluorescence was considered to represent damaged or dead cells, of which the cell membrane is permeable to PI. During cultivation 1 , the viability, which was calculated by dividing the number of living cells by the total number of cells, gradually decreased from $99 \pm 1 \%$ to $79 \pm 4 \%$ (Fig. 6.2A).
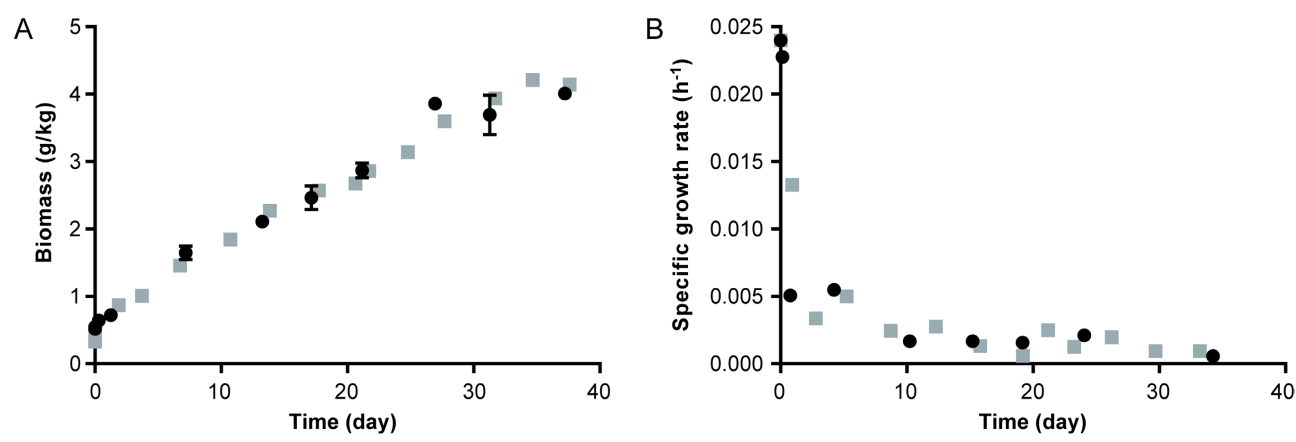

Figure 6.1: Growth of $L$. lactis FM03-V1 during retentostat cultivation. Circles and squares represent data points of retentostat cultivation 1 and 2 , respectively. At time zero a chemostat culture in steady state was switched to retentostat mode by insertion of a filter in the effluent line. A: measured biomass concentration; data points represent average \pm standard deviation of duplicate samples. B: Calculated specific growth rates based on the cell dry weight measurements. 
To determine the number of cells that remained culturable during the cultivations, serial dilutions were plated on LM17 plates. The plate counts hardly increased during the cultivations (Fig. 6.2B), while the biomass concentrations increased 8 -fold (Fig. 6.1A). This difference could not be explained by increased chain lengths of the bacteria because the average chain length even decreased approximately 2 -fold during the cultivation from 13 to 7 cells per chain (Suppl. Fig. S6.2). The above observations indicate that a large fraction of the bacteria remained viable but lost culturability on LM17 plates. The morphology of the retentostat-grown cells was examined using scanning electron microscopy (Fig. 6.3). This showed that the population became more heterogeneous during the cultivations and the fraction of cells with an altered appearance gradually increased. These cells were bigger or longer (more rod-shaped) (Suppl. Fig. S6.3). Moreover, the surface of these altered cells was rougher than that of fast growing cells and was comparable to the cell surface near the division plane of growing cells (compare cells at $\mathrm{t}=0$ and $\mathrm{t}=37$ in Fig. 6.3).
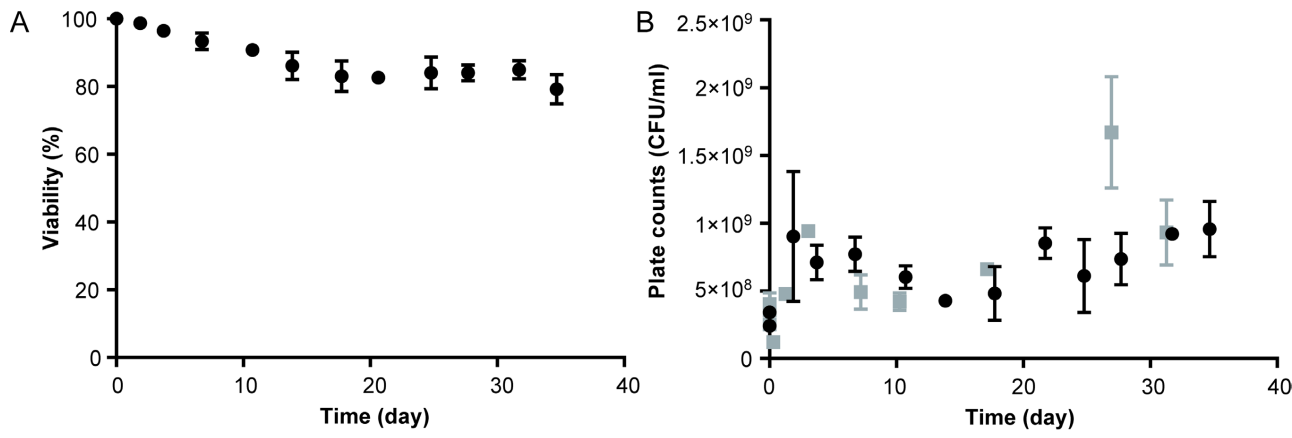

Figure 6.2: Culturable cells and viability of L. lactis FM03-V1 during retentostat cultivation. At time zero a chemostat culture in steady state was switched to retentostat mode by insertion of a filter in the effluent line. A: Viability of cells in retentostat cultivation 1 determined using SYTO 9 and propidium iodide as fluorescent markers followed by fluorescent microscopy and manual counting. Cells with strong green and weak red fluorescence were considered live and cells with weak green and strong red fluorescence were considered dead. Data points represent the average \pm standard deviation of at least five pictures taken from the same sample. B: Culturable cells from retentostat 1 (circle) and 2 (square) determined by plating on M17 supplemented with $0.5 \%$ lactose. Data points represent average \pm standard deviation of duplicate samples. 


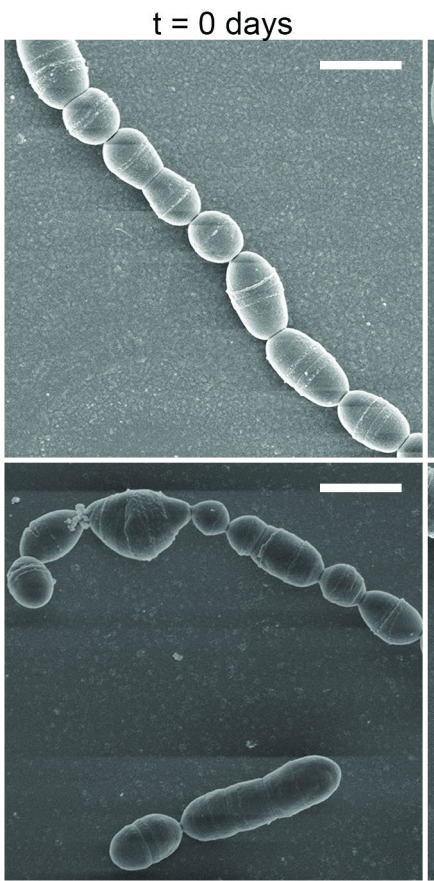

$\mathrm{t}=34$ days

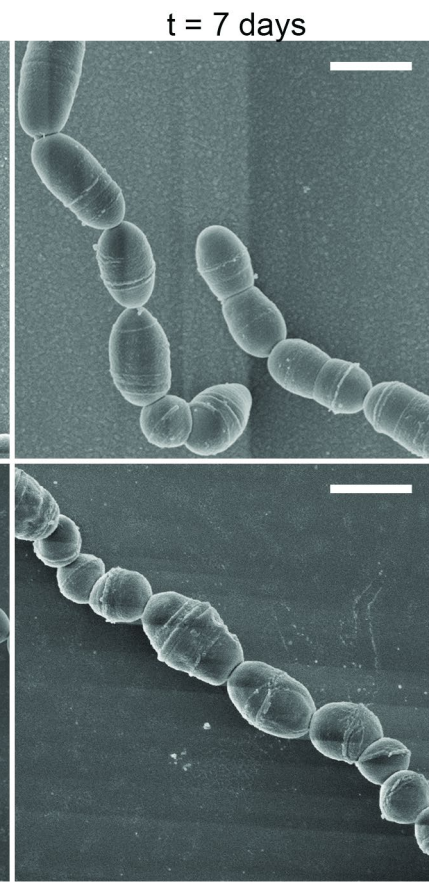

$\mathrm{t}=37$ days

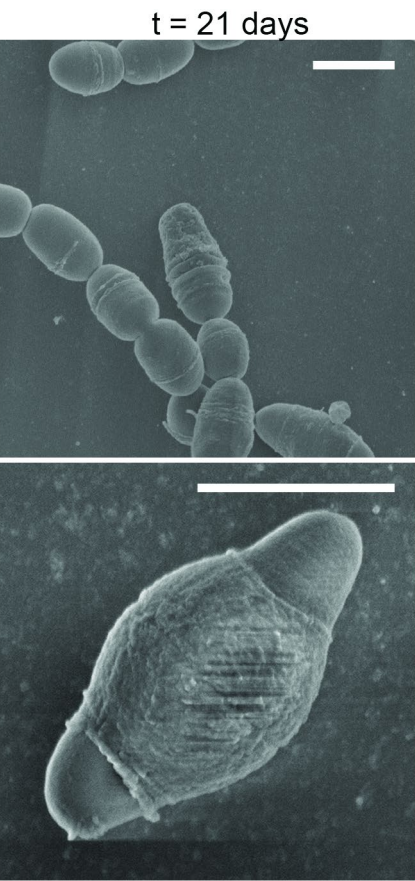

$\mathrm{t}=37$ days

Figure 6.3: Morphology of L. lactis FM03-V1 during cultivation in retentostat 1 analysed using scanning electron microscopy. Similar morphology was found for cells in retentostat 2. Samples were taken at different time points and stored at $-20^{\circ} \mathrm{C}$ until used for analysis by scanning electron microscopy. Morphology of cells was not affected by the freezing (data not shown). White bars correspond to $1 \mu \mathrm{m}$.

\section{Metabolism}

Concentrations of the main carbon sources (lactose and citrate) and the main fermentation products (lactate, acetate, ethanol, formate, acetoin and pyruvate) were determined with HPLC. In all cultivations no residual lactose and citrate was detected. Lactose and citrate were mainly converted into a mixture of lactate, formate, acetate and ethanol, but also small amounts of acetoin and pyruvate were detected. The product concentrations were converted into production rates using the effluent rate and compared with the consumption rates of lactose and citrate to determine how substrate was distributed over the various metabolic pathways of L. lactis (Fig. 6.4). 



Figure 6.4: Metabolite production by L. lactis FM03-V1 during retentostat cultivation. Left: Distribution of pyruvate over five different metabolic pathways during retentostat cultivation 1 and 2 . The distribution is normalised to $100 \%$. Every colour corresponds to a particular pathway highlighted in the right picture: LDH (purple), PFL (red), PDH (green), ALS (pink), pyruvate efflux (orange). Circles and squares represent the electron-balances of retentostat 1 and 2, respectively, which were calculated based on the degree of reduction of the main substrate and products. At time zero a chemostat culture in steady state was switched to retentostat mode by insertion of a filter in the effluent line. Right: Overview of the central metabolism of $L$. lactis FM03-V1 during growth on lactose and citrate according to stoichiometry. Reactions are normalised based on the production or consumption of 1 pyruvate or 1 acetyl-CoA. LDH: lactate dehydrogenase; PFL: pyruvate formate lyase; PDH: pyruvate dehydrogenase; ALS: acetolactate synthase; ALD: acetolactate decarboxylase; PTA: phosphotransacetylase; ACK: acetate kinase; ADH: alcohol dehydrogenase.

During the first 10 days of retentostat cultivation, lactate production via lactate dehydrogenase relatively increased from approximately $50 \%$ to $60-70 \%$, while acetate and ethanol production via acetyl-CoA forming reactions decreased from $40-50 \%$ to $30 \%$. This distribution remained constant during the rest of the cultivations. During retentostat cultivation 2, acetoin production decreased from $11 \%$ to approximately $2 \%$. In contrast, in retentostat cultivation 1 only $2 \%$ of the substrate was converted into acetoin throughout the cultivation. In both cultivations formate production rates were low compared to production rates of ethanol and acetate (even after correction for acetate production by citrate lyase). This indicates that pyruvate formate lyase ( $P F L$ ) was not the only enzyme producing acetyl-CoA and most likely pyruvate dehydrogenase $(\mathrm{PDH})$ was active despite the anaerobic conditions. 


\section{Maintenance requirements}

The maximum biomass yield on ATP ( $\left.Y_{\text {X/ATP }}{ }^{\max }\right)$ and the maintenance coefficient $\left(m_{\text {ATP }}\right)$ were estimated by fitting the biomass accumulation data to a modified Verseveld equation in which changes in metabolism were taken into account (Eq. 6.1). Two models were used: $i)$ both $Y_{X / A T P}{ }^{\max }$ and $m_{\text {ATP }}$ were constant and fitted and ii) $Y_{X / A T P}{ }^{\max }$ was constant and estimated from chemostat cultivations, while $\mathrm{m}_{\text {ATP }}$ was fitted and linearly dependent on the biomass specific ATP production rate $\left(q_{A T P}\right)$ with a maximum $m_{\text {ATP }}$ max. The models were compared based on $\left.i\right)$ how well they fitted the biomass accumulation, ii) the estimated biomass concentrations in the chemostat phase and iii) biological interpretation of changes in $Y_{X / A T P} \max$ and $m_{\text {ATP. }}$. The biomass concentration in the chemostat was estimated using equation 6.4 (derivation in Supplement B).

$$
C_{x, \text { chemostat }}=\frac{D \cdot\left(C_{s, i n}-C_{S, o u t}\right) \cdot Y_{A T P / S}}{\frac{D}{Y_{x / A T P}^{m a x}}+m_{A T P}}
$$

Both models fitted the biomass accumulation very well, but only for model 2 the obtained $\mathrm{Y}_{\mathrm{X} / \mathrm{ATP}} \max$ and $\mathrm{m}_{\text {ATP }}$ could also predict the biomass concentration in the chemostat phase $(\sim 0.6 \mathrm{gDW} / \mathrm{kg}$ ) (Table 6.1 and Suppl. Fig. S6.4). Moreover, model 1 predicted a 4 times lower $\mathrm{Y}_{\mathrm{X} / \mathrm{ATP}}{ }^{\max }$ during retentostat cultivation compared to chemostat cultivation, which is unlikely because the $Y_{X / A T P}{ }^{\max }$ is assumed to be a constant growth parameter when using the same medium and environmental conditions. In contrast, model 2 assumed the same $Y_{X / A T P} \max$ as in chemostat cultivations and predicted that the maintenance requirement gradually decreased and approached a minimum of $0.36 \pm 0.03 \mathrm{mmol}$ ATP.gDW-1. $\mathrm{h}^{-1}$.

It has been suggested that microorganisms can adapt to situations of extreme calorie restriction and lower their maintenance requirements (Kempes et al., 2017). We conclude that model 2 better describes the physiological changes during retentostat cultivation, which suggests that the maintenance coefficient of our $L$. lactis strain decreases at near-zero growth rates. Using model 2 , the growth rate was predicted and plotted against the biomass specific ATP production rate ( $\left.\mathrm{q}_{\text {ATP }}\right)$ and compared with data from chemostat cultivations used to estimate the $\mathrm{Y}_{\mathrm{X} / \mathrm{ATP}}{ }^{\max }$ and $\mathrm{m}_{\text {ATP }}$ (Fig. 6.5). This shows that these estimates are only valid at growth rates above approximately $0.01 \mathrm{~h}^{-1}$. At near-zero growth rates, the ATP required for growth is significantly lower than predicted from the chemostat cultivations due to the decreasing maintenance requirements. 


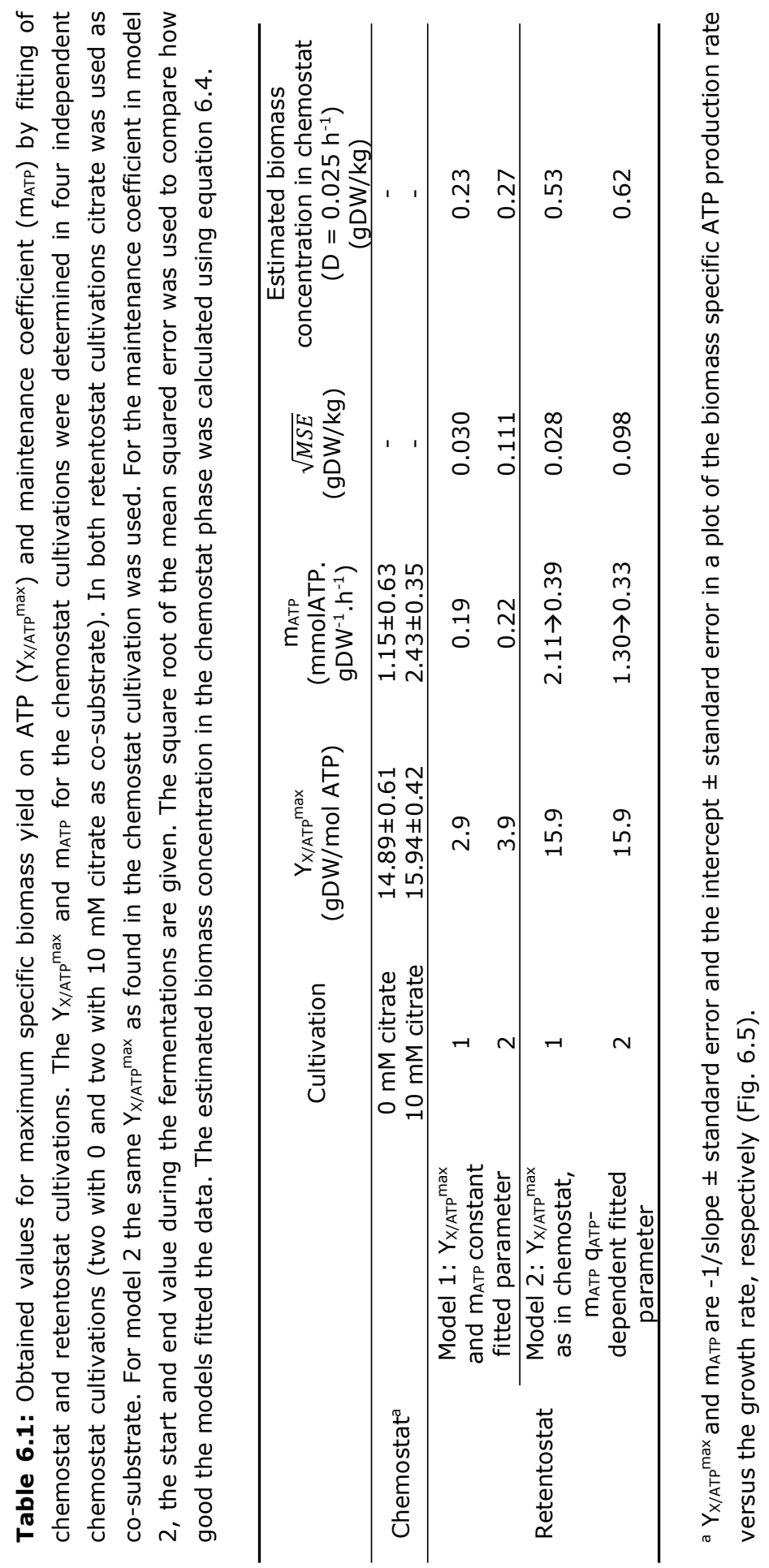




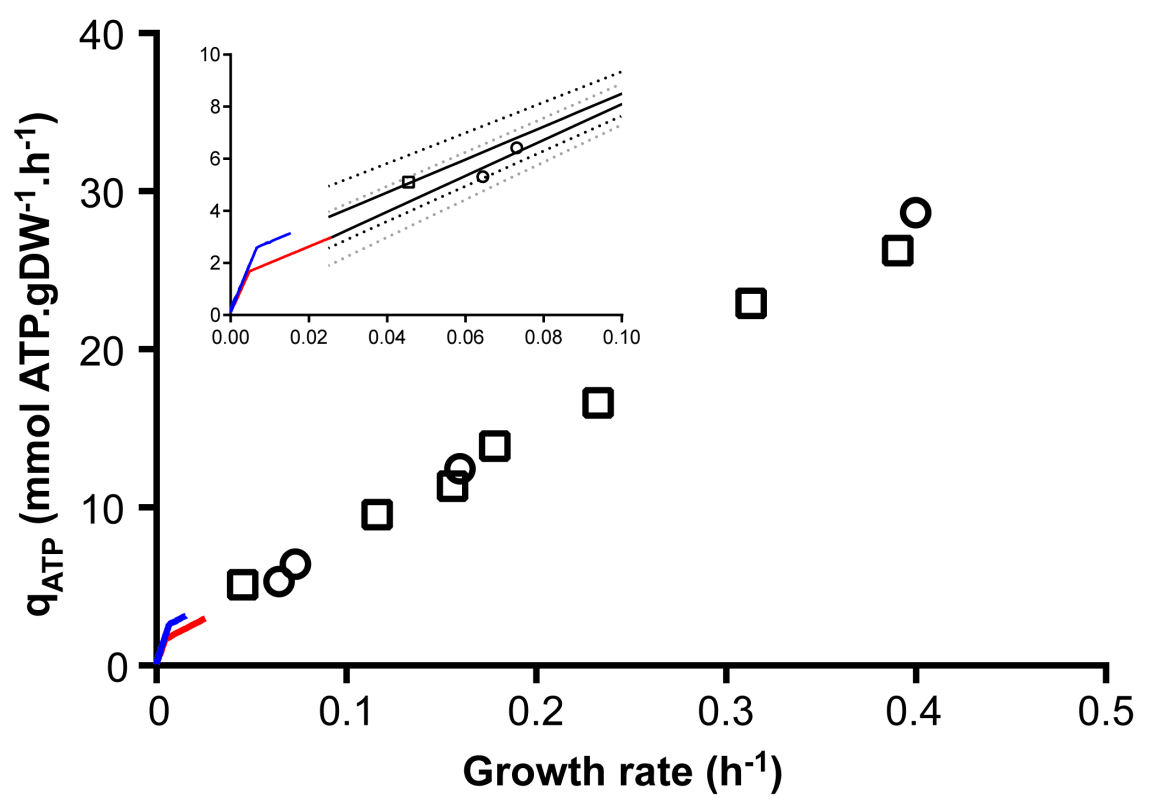

Figure 6.5: Relation between the specific growth rate and the biomass specific ATP production rate $\left(q_{A T P}\right)$ in chemostat and retentostat cultivations. Data from chemostat cultivations with and without citrate as co-substrate are represented by squares and circles, respectively. The black lines in the inset represent linear regression lines of the chemostat data points used to estimate the maximum biomass yield on ATP and the maintenance coefficient. Dotted lines in the inset represent the $95 \%$ confidence intervals of these linear regression lines. The biomass specific ATP production rate and growth rate in retentostat cultivations 1 and 2 were calculated based on biomass predictions of model 2 and shown by blue and red lines, respectively. Axis titles in the inset are the same as the main figure.

\section{Maintenance components}

Kempes et al. (2017) determined for a wide range of cell sizes what the major components of the maintenance requirements are. For $L$. lactis with a volume of about $5.10^{-19} \mathrm{~m}^{3}$ (sphere with a diameter of $1 \mu \mathrm{m}$ ) it is expected that protein repair and maintaining trans-membrane proton gradients are the major constituents of the maintenance and other components, like RNA turnover, play a minor role. Due to the smaller surface to volume ratio in bigger cells, proton leakage is generally smaller in bigger cells. Both in this study and in retentostat studies with the plant-associated L. lactis KF147 the cell size increased (Ercan et al., 2013). However, the cell size decreased for microorganisms upon starvation (Lever et al., 2015). This suggests a different response towards starvation and near-zero growth conditions. 

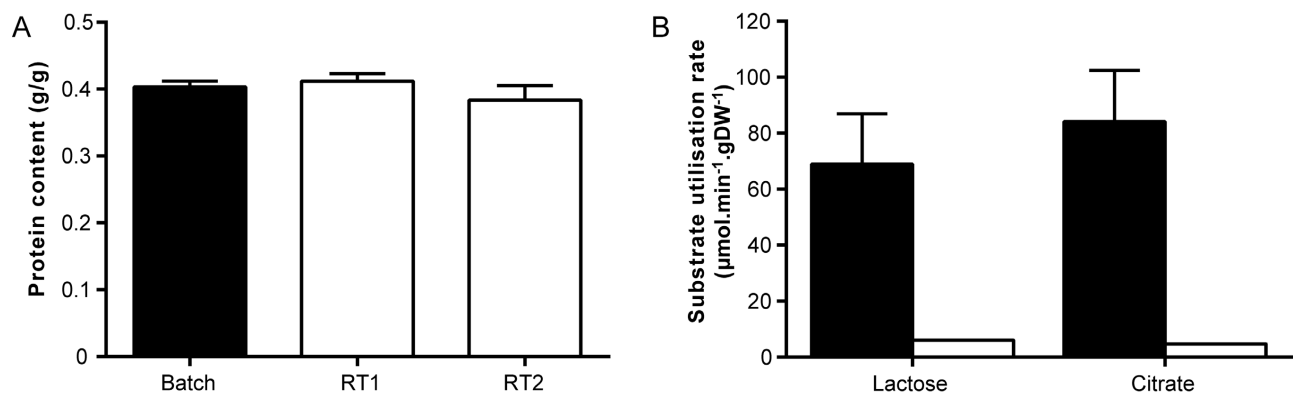

Figure 6.6: Protein content and substrate utilisation rate of L. lactis FM03-V1 grown on chemically defined medium with citrate in either batch (filled bars) or retentostat cultures (open bars). Cells were grown in three independent batch cultures and were harvested in exponential phase. For the retentostat cultivations, cells were harvested at the end of the cultivation (37 days). A: Protein content. RT1 and RT2 represent retentostat cultivation 1 and 2, respectively. Error bars represent the standard deviation of triplicate measurements. B: Substrate utilisation rates of lactose and citrate. Only retentostat culture 1 has been analysed. The error bars represent the standard deviation of biological triplicates.

One way for a cell to minimise its energy need for protein repair is to decrease its protein content. The protein content was determined at the end of the retentostat cultivations and compared with the protein content of exponentially growing cells. The protein content was not significantly different between fast and slow growing cell cultures suggesting that the bacteria adapted in a different way to extreme calorie restriction (Fig. 6.6A).

The in vivo utilisation capacity of lactose and citrate of retentostat-grown cells was compared with exponentially grown cells to see adaptation at the level of enzyme capacities. The lactose and citrate utilisation capacity were 11 and 18fold lower in the retentostat-grown cells, respectively (Fig. 6.6B). This suggests that the bacteria might have adapted to the extreme calorie restriction by reducing the repair and/or replacement of damaged proteins resulting in a similar protein content but less functional proteins.

\section{Aroma formation during retentostat cultivation}

During the retentostat cultivations, samples were taken to monitor the formation of volatile organic compounds (VOCs) using headspace solid phase microextraction gas chromatography (HS SPME GC-MS). Samples were analysed without removal of cells because particular aromas might be located in the cells due to their hydrophobic nature. In total 62 compounds were identified. Comparison of peak areas of each compound in retentostat samples and medium samples, revealed that 56 compounds were higher in concentration in the 
retentostat samples and therefore considered to be produced during the cultivation. The produced compounds were assigned to different groups of chemicals: acids, alcohols, aldehydes, ketones, esters, sulphur-containing compounds, pyrazines, and other compounds (Suppl. Table S6.1, Fig. 6.7). The compounds mainly originated from primary metabolism and the degradation of amino acids (methionine, isoleucine, leucine, phenylalanine, threonine and tyrosine) and fatty acids.

The abundance of 14 compounds significantly changed in time and thus were affected by the low growth rates. These compounds include products of amino acid degradation (benzaldehyde, benzeneacetyladehyde, 3-(methylthio)-propanal and acetophenone), products of degradation of medium and long chain fatty acids (octanoic acid, 2-heptanone, tridecanal, 1-tetradecanol, 1-hexadecanol and decanoic acid, ethyl ester), and some other compounds (3-methyl-2-butenal, 2,3-dimethylpyrazine, 2,5-dimethylpyrazine, 3-methyl-1-hexanol). All of these compounds increased in time (Fig. 6.8), which suggests that production of these compounds increases in cells that are growing very slowly.
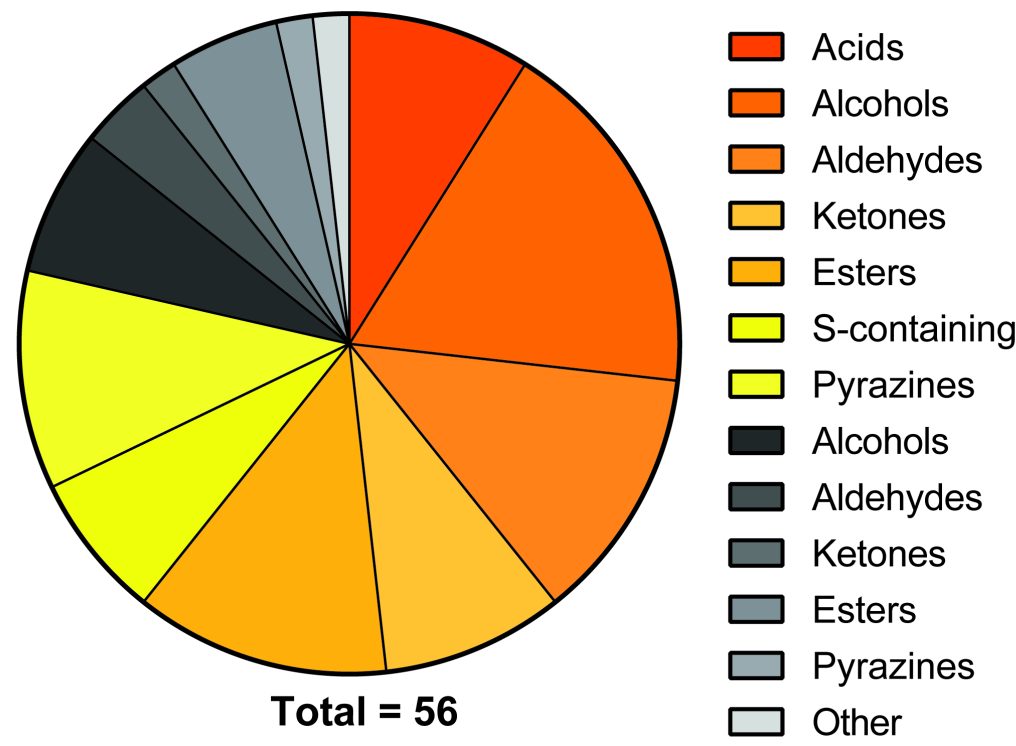

Figure 6.7: Composition of the volatile organic compounds produced by L. lactis during retentostat cultivations. Every pie sector corresponds to a group of compounds (Suppl. Table 6.1) and the colour represents if the group is found in cheese (orange to yellow) or not (grey). Five studies describing aroma compounds in various cheeses, including Gouda, Cheddar, Parmigiano-Reggiano and Parmasan, were selected for comparison (Barbieri et al., 1994, Curioni and Bosset, 2002, Qian and Reineccius, 2002, Singh et al., 2003, van Leuven et al., 2008). 


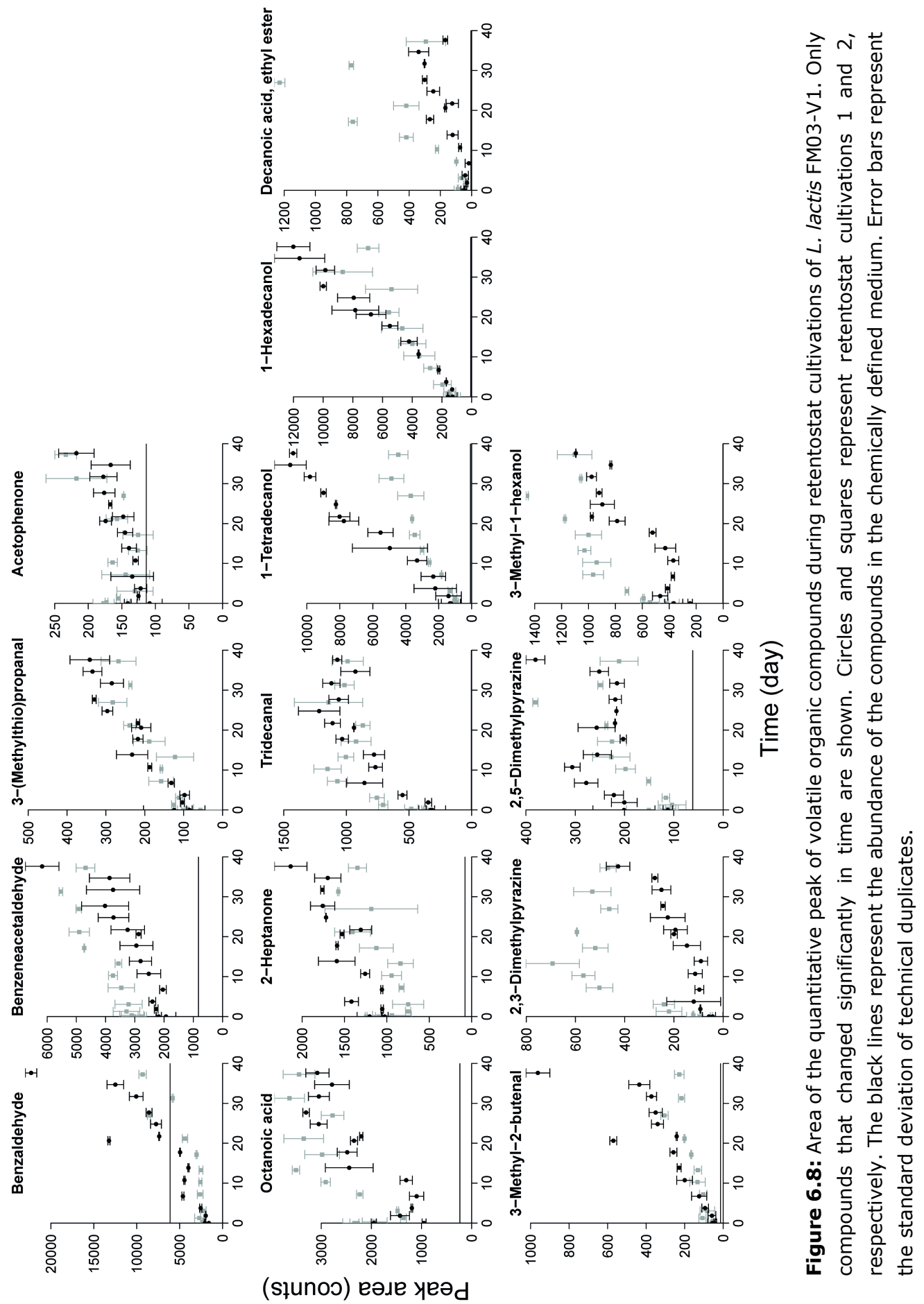


Fat-derived compounds play a crucial role to achieve a balanced cheese flavour (Urbach, 1993). Although milk fat and fatty acids were not present in the chemically defined medium, some fat-related compounds were produced (Suppl. Table S6.1) and some even increased during the cultivations (tridecanal, 1-tetradecanol, 1-hexadecanol and decanoic acid, ethyl ester) (Fig. 6.8). These compounds might originate from the turnover or degradation of phospholipids of the cell membrane. Interestingly, these compounds were hardly detected in cellfree samples in which cells were removed by centrifugation (data not shown). This shows that these compounds were associated with cells.

\section{Partitioning of VOCs}

To get further insight in the partitioning of compounds, we compared the VOC profiles of complete samples (cells and extracellular liquid) and cell-free samples (only extracellular liquid; cells removed by centrifugation). Sixteen compounds were significantly more abundant in the complete samples (Suppl. Fig. S6.5) indicating that significant fractions of these compounds were located in the cells. More hydrophobic compounds were relatively more abundant in the cells (Fig. 6.9) suggesting that the hydrophobic compounds predominantly accumulated in the cell membrane. Because cells were retained in the retentostat cultivation, also these aroma compounds were retained, which explains why some fat-related compounds increased in time (Fig. 6.8).

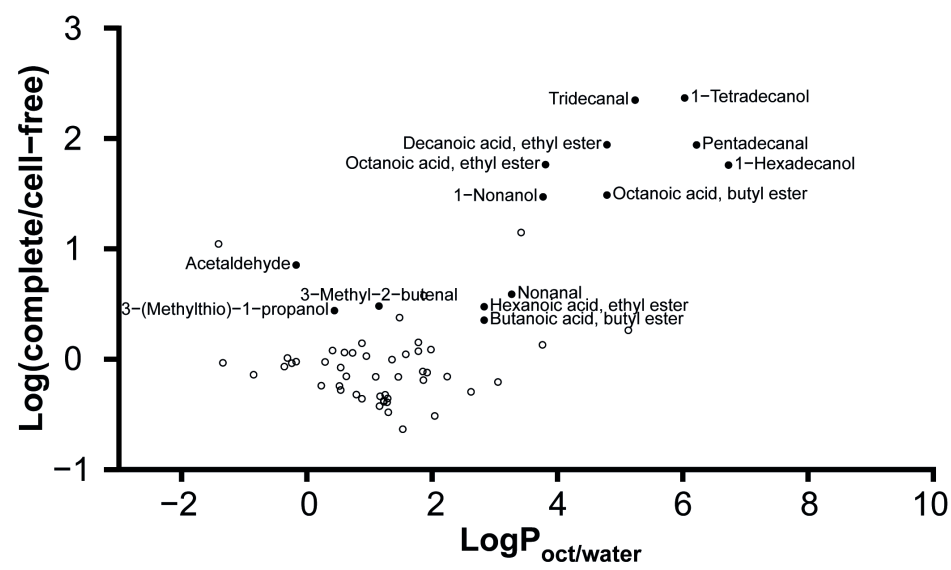

Figure 6.9: Abundance of compounds in complete samples and cell-free samples as function of their hydrophobicity (logPoct/water). Filled circles represent compounds that were significantly more abundant in complete samples and their names are given. Open circles represent compounds that were not significantly more abundant in complete samples. Data of both cultivations was taken into account by plotting the abundances in complete and cellfree samples (Suppl. Fig. S6.5), calculating the slopes and taking the logarithm of these slopes. 


\section{Discussion}

\section{Physiology of L. lactis at near-zero growth rates}

The physiology of a dairy Lactococcus lactis subsp. lactis biovar diacetylactis growing at near-zero growth rates has been investigated by inducing extreme carbon and energy limitation using retentostat cultivation. Biomass accumulation, culturability, viability, morphology and metabolite production were analysed revealing physiological adaptations to near-zero growth rates. Moreover this is, to the best of our knowledge, the first study of aroma formation during the retentostat cultivations revealing changes in the secondary metabolism of this bacteria upon extreme calorie restriction.

During 37 days of retentostat cultivation, the biomass concentration increased 8 -fold while the growth rate decreased till less than $0.001 \mathrm{~h}^{-1}$. Compared to the plant-associated L. lactis KF147, biomass accumulated much slower and no plateau was reached within 37 days. This was most likely caused by adaptation of the strain to the near-zero growth rates resulting in decreased maintenance costs. Decreasing maintenance costs during retentostat cultivation has also been described for Pichia pastoris and Pseudomonas putida (Panikov et al., 2015, Rebnegger et al., 2016). After 37 days of retentostat cultivation, the estimated maintenance coefficient of $L$. lactis FM03-V1 was approximately 7 -fold lower than the value observed during chemostat cultivation and was similar to the maintenance coefficient of $L$. lactis KF147 (1.04 \pm 0.07 and $1.11 \pm 0.04$ mCmolS.gDW-1. $\mathrm{h}^{-1}$, respectively) (Ercan et al., 2013). Kempes et al. (2017) combined data about maintenance costs from a very broad range of organisms and concluded that protein repair and proton leakage are the main components of the maintenance costs for organisms with a diameter of approximately $1 \mu \mathrm{m}$. Lahtvee et al. (2014) measured protein turnover rates in L. lactis and showed that protein turnover accounts for the highest maintenance costs in L. lactis. Moreover, they showed that the protein turnover rate decreases at lower growth rates. In our study the capacity to utilise lactose and citrate significantly decreased in retentostat-grown cells compared to exponential growing cells, while the protein content remained constant. In a retentostat study with Lactobacillus plantarum the protein content also remained constant (Goffin et al., 2010). We speculate that the bacteria might have adapted to the nutrient limitation by decreasing the protein turnover (i.e. repair and replacement of damaged proteins). This would result in less functional proteins (lower capacity), while the amount of proteins will remain the same (same protein content) and could greatly reduce the energy required for protein repair. Such response has also been observed for Bacillus 
subtilis and Saccharomyces cerevisiae, which showed a reduced expression of the translational machinery during retentostat cultivation (Boender et al., 2011, Overkamp et al., 2015).

Comparing the biomass yield on substrate and maintenance coefficient of $L$. lactis FM03-V1 to other strains revealed that this strain, when grown in chemostat cultures, has very similar growth parameters as other dairy L. lactis strains (Adamberg et al., 2003, Meghrous et al., 1992, Otto et al., 1980, Ten Brink et al., 1985, Thomas et al., 1979). The low maximum biomass yield on substrate in combination with the high maintenance costs of dairy strains suggests adaptation towards the nutrient-rich dairy environment. In contrast, the plant-associated environment is commonly classified as a poor condition for microbial growth due to low $\mathrm{pH}$, high osmolarity and/or low nutrient conditions and therefore the plantisolated L. lactis KF147 may have evolved to these harsh environments by minimising its maintenance costs (Ercan et al., 2013).

During the cultivations, a significant fraction of the population lost the ability to grow on LM17 plates, while maintaining a high viability as determined by staining with SYTO 9 and propidium iodide. The increase in viable but non-culturable cells (VBNC) has also been observed for Pseudomonas putida during retentostat cultivation and was linked to a decreased ribosome content in VBNC cells (Panikov et al., 2015). Also during cheese ripening L. lactis loses the capability to grow on M17 plates, while remaining its transcriptional activity (Desfossés-Foucault et al., 2013, Ruggirello et al., 2014, Ruggirello et al., 2016). A relative increase in VBNC cells during cheese ripening was also found by quantification of culturable cells (by plate counting) and viable cells (using qPCR with a propidium monoazide treatment) (Erkus et al., 2013, Erkus et al., 2016).

Based on the metabolite production profile, we concluded that despite the anaerobic conditions pyruvate dehydrogenase was active and responsible for part of the conversion of pyruvate into acetyl-CoA. In $L$. lactis, pyruvate dehydrogenase (PDH) is highly sensitive for the NADH/NAD ratio, which prevents in vivo activity under aerobic conditions (Condon, 1987, Snoep et al., 1992, Snoep et al., 1993), but apparently a small activity remained under anaerobic conditions. The highest flux through PDH was $0.26 \mathrm{mmol} . \mathrm{gDW}^{-1} . \mathrm{h}^{-1}$ which is lower than found as in vitro activity for PDH for L. lactis MG1363 grown under anaerobic conditions at a dilution rate of $0.1 \mathrm{~h}^{-1}$ (Jensen et al., 2001). This indicates that PDH activity was in absolute terms not high in this experiment, but relatively abundant because all other metabolic fluxes were also very low in retentostat cultivation. This allows relatively low metabolic fluxes to contribute significantly to the overall metabolism during retentostat cultivation. 


\section{Aroma formation in retentostat cultures resembles aroma formation during cheese ripening}

Volatile organic compounds (VOCs) were analysed to detect changes in the secondary metabolism of the bacterium and to compare aroma formation at nearzero growth rates and during cheese ripening. Of the VOCs that were produced during the retentostat cultivations, $79 \%$ can be found in ripened cheese (Suppl. Table S6.1). Production of particular aroma compounds increased at near-zero growth rates including some specific cheese flavours thereby resembling reactions occurring during cheese ripening.

Degradation of amino acids is a major source of flavour compounds in cheese (Yvon and Rijnen, 2001). In the retentostat cultivations in total 12 amino acid degradation products were identified that could originate from degradation of phenylalanine, tyrosine, threonine, methionine, leucine and isoleucine. The abundance of some of these products increased significantly in time (benzaldehyde, benzeneacetaldehyde, 3-(methylthio)propanal and acetophenone) indicating that production of these compounds was higher in a culture growing very slowly. Although products of branched chain amino acids degradation are important flavours produced by $L$. lactis during cheese ripening, production of these compounds was limited. This can be explained by the large deletion in the gene encoding the branched chain a-keto acid decarboxylase $(k d c A)$ in L. lactis FM03-V1 as also described for L. lactis IL1403 (Smit et al., 2005a).

Ketones are common constituents of cheese and especially methyl ketones are known for their contribution to the aroma of blue-veined and surface mouldripened cheese (Curioni and Bosset, 2002). 2-Heptanone has a blue cheese note and its formation in surface-ripened cheese has been related to the enzymatic activity of moulds, which convert octanoic acid into 2-heptanone (Gehrig and Knight, 1961). In the retentostat cultures both octanoic acid and 2-heptanone increased in time suggesting that also $L$. lactis is able to produce 2-heptanone and that production of 2-heptanone is increased in cells growing at near-zero growth rates. During retentostat cultivation 1, 2-butanone increased 9-fold, while the acetoin concentration remained low (Suppl. Fig. S6.6). Acetoin might have been continuously produced, but further converted into 2-butanone resembling reactions during cheese ripening (Keen et al., 1974). This would also explain the higher abundance of 2-butanone in retentostat 2 in which slightly more acetoin was produced at the onset of the cultivation (Suppl. Fig. S6.6). 
During the retentostat cultivations several ethyl and butyl esters increased in time. Butyl esters can be formed by condensation of a carboxylic acid with 1-butanol, which was present in high concentrations in the medium as part of the Bacto-tryptone, and are generally not found in cheese. Most common in cheese are ethyl esters of straight-chain fatty acids of $\mathrm{C2}-\mathrm{C} 10$, which could be formed enzymatically by the bacteria by condensation of ethanol and a carboxylic acid (Curioni and Bosset, 2002, Liu et al., 2004a). These ethyl esters are responsible for the fruitiness of Italian-type cheeses, but at high concentrations they result in a fruity off-flavour. In general, ethanol is the limiting factor in ester synthesis in cheese (Liu et al., 2004a, Thierry et al., 2006). In contrast, the ethanol concentration in the retentostat cultures was high due to the mixed acid fermentation behaviour of the culture, which resulted in formation of various esters. In L. lactis FM03-V1 the estA gene encodes an alcohol acyltransferase, which might be responsible for the ester formation (Liu et al., 2004b, Nardi et al., 2002).

In all retentostat cultivations 3-methyl-2-butenal increased in time. The odour of this compound has been described as almond (Burdock, 2010) and as cheese, fruity and green (Curioni and Bosset, 2002) and this compound is sometimes found in cheese (Gogus et al., 2006, Majcher et al., 2010, Majcher et al., 2011) and yoghurt (Cheng, 2010). Based on metabolism of L. lactis, we speculate that this compound is chemically produced from dimethylallyl pyrophosphate (DMAPP), which is the end-product of the mevalonate pathway required for isoprenoid synthesis. Therefore, the increase in 3-methyl-2-butenal suggests accumulation of DMAPP caused by the near-zero growth rates.

Several pyrazines were produced during the retentostat cultivations. Pyrazines have an earthy, roasty odour and are important contributors to cheese flavour, especially in Cheddar and Gruyère (Curioni and Bosset, 2002). The pyrazines found in this study can be linked to enzymatic reactions involving diketones, such as 2,3-butanedione (diacetyl) and 2,3-pentanedione (Dickschat et al., 2010).

Although fat and fatty acids were not present in the chemically defined medium, some fat-related compounds were produced and even increased during the retentostat cultivations. Most likely these compounds originated from the turnover of phospholipids of the cell membrane or from degradation of phospholipids of dead cells. Most of the fat-related compounds are hydrophobic and therefore accumulate in the lipid bilayer of the cytoplasmic membrane. Because cells were retained in the bioreactor, also these hydrophobic compounds were largely retained and also accumulated in time. 
In conclusion, this study shows that retentostat cultivation is a unique tool to study microorganisms under industrially relevant conditions of slow growth. Physiological responses of $L$. lactis subsp. lactis biovar diacetylactis in the retentostat cultivations show similarities with the in situ behaviour of this lactic acid bacterium during cheese ripening e.g. viable but non-culturable cells and characteristic aroma formation. The similarities in aroma formation between lactococcal cells captured in the cheese matrix and cells in a retentostat bioreactor, indicates that retentostat cultivation might not only be used as study platform, but also as production platform for specific cheese aromas.

\section{Acknowledgements}

This work was supported by Arla Foods (Aarhus, Denmark). We thank Judith Wolkers-Rooijackers and Irma van Rijswijck for their assistance in the GC-MS analysis. We thank Marcel Giesbers (Wageningen Electron Microscopy Centre, Wageningen University \& Research) for his assistance in SEM. 


\section{Supplementary materials}

Supplementary Table S6.1: Volatile organic compounds produced by L. lactis during retentostat cultivation and their putative origin. Compounds in bold can be found in cheese (Barbieri et al., 1994, Curioni and Bosset, 2002, Qian and Reineccius, 2002, Singh et al., 2003, Van Leuven et al., 2008). Compounds with an asterisk significantly increased during the retentostat cultivations. The number of compounds per group and their presence in cheese has been is summarised in Figure 6.8.

\begin{tabular}{|c|c|c|c|}
\hline Group & Compound & Putative origin & Reference \\
\hline Acid & $\begin{array}{c}\text { Acetic acid } \\
\text { Butanoic acid } \\
\text { Hexanoic acid } \\
\text { Octanoic acid* } \\
\text { Nonanoic acid } \\
\end{array}$ & $\begin{array}{l}\text { Primary metabolism } \\
\text { Fatty acid biosynthesis } \\
\text { Fatty acid biosynthesis } \\
\text { Fatty acid biosynthesis } \\
\text { Fatty acid biosynthesis }\end{array}$ & \\
\hline \multirow{4}{*}{ Alcohol } & $\begin{array}{l}\text { Ethanol } \\
\text { 2-Methyl-1-butanol } \\
\text { 3-Methyl-1-butanol }\end{array}$ & $\begin{array}{l}\text { Primary metabolism } \\
\text { Isoleucine } \\
\text { Leucine }\end{array}$ & $\begin{array}{l}\text { (Yvon and Rijnen, 2001) } \\
\text { (Yvon and Rijnen, 2001) }\end{array}$ \\
\hline & $\begin{array}{l}\text { 3-Methyl-3-buten-1-ol } \\
\text { 3-Methyl-2-buten-1-ol }\end{array}$ & $\begin{array}{c}\text { IPP } \\
\text { (mevalonate pathway) } \\
\text { DMAPP } \\
\text { (mevalonate pathway) }\end{array}$ & $\begin{array}{l}\text { (This study) } \\
\text { (This study) }\end{array}$ \\
\hline & Benzylalcohol & Phenylalanine & $\begin{array}{l}\text { (Nierop Groot and de } \\
\text { Bont, 1998) }\end{array}$ \\
\hline & $\begin{array}{c}\text { 2-Phenylethanol } \\
\text { Phenol } \\
\text { 1-Nonanol } \\
\text { 1-Dodecanol } \\
\text { 1-Tetradecanol* } \\
\text { 1-Hexadecanol* } \\
\text { 3-Methyl-1-hexanol* } \\
\text { 1-(4-Methylphenyl)ethanol }\end{array}$ & $\begin{array}{l}\text { Phenylalanine } \\
\text { Tyrosine } \\
\text { Fatty acid } \\
\text { Phospholipid } \\
\text { Phospholipid } \\
\text { Phospholipid } \\
\text { - } \\
\text { - }\end{array}$ & $\begin{array}{l}\text { (Yvon and Rijnen, 2001) } \\
\text { (Yvon and Rijnen, 2001) } \\
\text { (This study) } \\
\text { (This study) } \\
\text { (This study) }\end{array}$ \\
\hline \multirow{9}{*}{ Aldehyde } & Acetaldehyde & Threonine / Glycine & (Collins et al., 2003) \\
\hline & 2-Butenal & $\begin{array}{l}\text { Acetaldehyde (aldol } \\
\text { condensation) }\end{array}$ & $\begin{array}{l}\text { (Hashimoto and } \\
\text { Kuroiwa, 1975) }\end{array}$ \\
\hline & 3-Methyl-2-butenal* & $\begin{array}{l}\text { DMAPP (mevalonate } \\
\text { pathway) }\end{array}$ & (This study) \\
\hline & Benzaldehyde* & $\begin{array}{l}\text { Phenylalanine / } \\
\text { Tryptophan }\end{array}$ & $\begin{array}{l}\text { (Singh et al., 2003, } \\
\text { Yvon and Rijnen, 2001) }\end{array}$ \\
\hline & Benzeneacetaldehyde* & Phenylalanine & (Yvon and Rijnen, 2001) \\
\hline & Nonanal & Fatty acid & (Collins et al., 2003) \\
\hline & Decanal & Fatty acid & (Collins et al., 2003) \\
\hline & Tridecanal* & Phospholipid & (This study) \\
\hline & Pentadecanal & Phospholipid & (This study) \\
\hline \multirow{4}{*}{ Ketone } & 2-Butanone & Fatty acid or acetoin & (Keen et al., 1974) \\
\hline & $\begin{array}{c}\text { Acetoin } \\
\text { (3-hydroxy-2-butanone) }\end{array}$ & Primary metabolism & \multirow{3}{*}{$\begin{array}{c}\text { (Ott et al., 2000) } \\
\text { (Ott et al., 2000) } \\
\text { (Gehrig and Knight, } \\
1961 \text { ) } \\
\text { (Singh et al., 2003) }\end{array}$} \\
\hline & $\begin{array}{c}\text { Diacetyl (2,3-butanedione) } \\
2,3 \text {-Pentanedione }\end{array}$ & $\begin{array}{c}\text { Primary metabolism } \\
\text { Threonine }\end{array}$ & \\
\hline & $\begin{array}{l}\text { 2-Heptanone* } \\
\text { Acetophenone* }\end{array}$ & $\begin{array}{l}\text { Octanoic acid } \\
\text { Phenylalanine }\end{array}$ & \\
\hline
\end{tabular}

Ester Formic acid, ethyl ester Formic acid, butyl ester Acetic acid, ethyl ester Acetic acid, butyl ester
Formic acid + ethanol

Formic acid + butanol

Acetic acid + ethanol

Acetic acid + butanol 


\begin{tabular}{|c|c|c|c|}
\hline & $\begin{array}{l}\text { Butanoic acid, ethyl ester } \\
\text { Butanoic acid, butyl ester } \\
\text { Hexanoic acid, ethyl ester } \\
\text { Octanoic acid, ethyl ester } \\
\text { Octanoic acid, butyl ester } \\
\text { Decanoic acid, ethyl ester* }\end{array}$ & $\begin{array}{l}\text { Butanoic acid + ethanol } \\
\text { Butanoic acid + butanol } \\
\text { Hexanoic acid }+ \text { ethanol } \\
\text { Octanoic acid }+ \text { ethanol } \\
\text { Octanoic acid }+ \text { butanol } \\
\text { Decanoic acid + ethanol }\end{array}$ & \\
\hline $\begin{array}{l}\text { Sulp } \\
\text { conta }\end{array}$ & $\begin{array}{c}\text { 3-(Methylthio)-1-propanol } \\
\text { 3-(Methylthio)-1-propanal* } \\
\text { Dimethyltrisulfide } \\
\text { Dimethyl sulfone }\end{array}$ & $\begin{array}{l}\text { Methionine } \\
\text { Methionine } \\
\text { Methionine } \\
\text { Methionine }\end{array}$ & $\begin{array}{l}\text { (Yvon and Rijnen, 2001) } \\
\text { (Yvon and Rijnen, 2001) } \\
\text { (Yvon and Rijnen, 2001) }\end{array}$ \\
\hline Pyr & $\begin{array}{l}\text { 2,3-Dimethylpyrazine* } \\
\text { 2,5-Dimethylpyrazine* } \\
\text { 2,6-Dimethylpyrazine } \\
\text { Trimethylpyrazine } \\
\text { Tetramethylpyrazine } \\
\text { 2-Ethyl-6-methylpyrazine } \\
\text { Trimethylethylpyrazine }\end{array}$ & $\begin{array}{c}\text { Acetoin + glyoxal } \\
\text { Methylglyoxal } \\
\text { Methylglyoxal } \\
\text { Acetoin }+ \text { methylglyoxal } \\
\text { Acetoin } \\
\text { Methylglyoxal }+ \\
\text { 2-oxo-butanal } \\
\text { Acetoin }+ \\
\text { 2,3-pentadione }\end{array}$ & $\begin{array}{l}\text { (Dickschat et al., 2010) } \\
\text { (Dickschat et al., 2010) } \\
\text { (Dickschat et al., 2010) } \\
\text { (Dickschat et al., 2010) } \\
\text { (Dickschat et al., 2010) } \\
\text { (Dickschat et al., 2010) } \\
\text { (Dickschat et al., 2010) }\end{array}$ \\
\hline Other & Trimethyloxazole & Acetoin + acetic acid & (Dickschat et al., 2010) \\
\hline
\end{tabular}




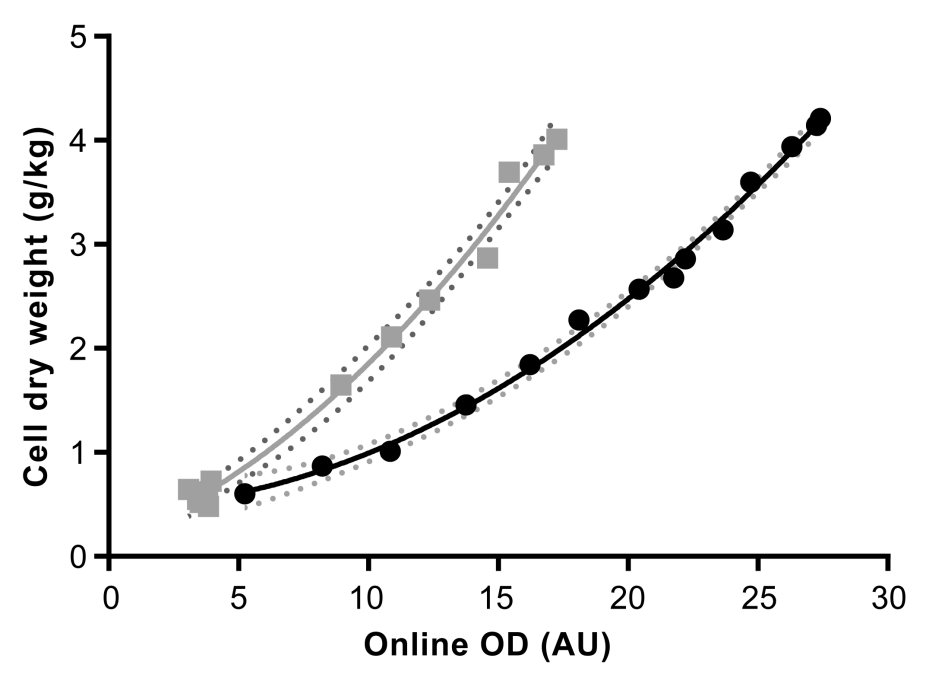

Supplementary Figure S6.1: Relation between optical density at $600 \mathrm{~nm}$ measured by an internal probe and the biomass concentration during two independent retentostat cultivation. The $95 \%$ confidence interval is given with the dotted lines. Circles and squares represent retentostat 1 and 2, respectively.

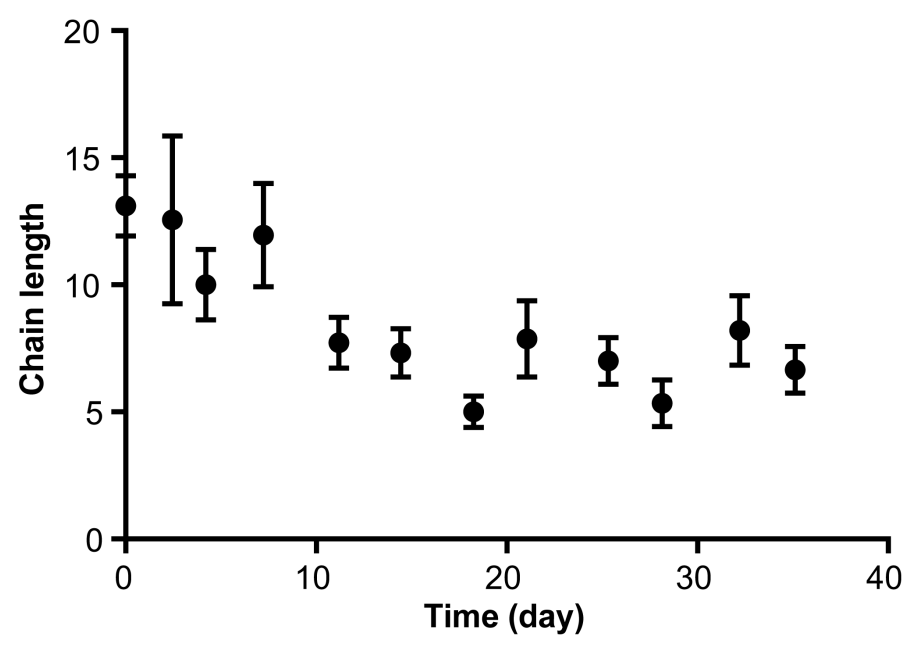

Supplementary Figure S6.2: Average chain length during retentostat cultivation 1 . The chain length of 19 to 66 chains per sample was determined by manually counting of cells on microscopic pictures. Chains had similar lengths in retentostat cultivation 2. At time zero a chemostat culture in steady state was switched to retentostat mode by insertion of a filter in the effluent line. Error bars represent the standard error. 


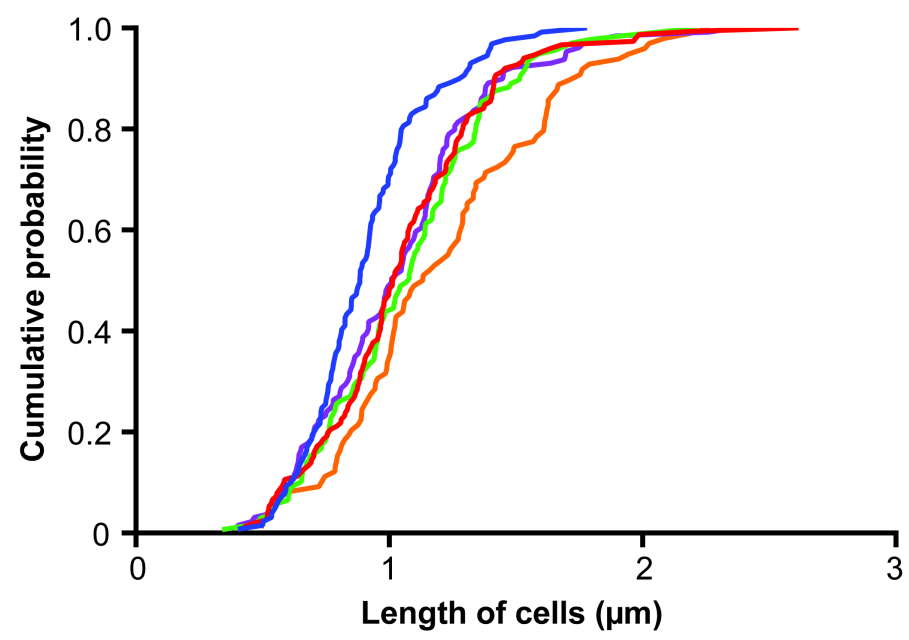

Supplementary Figure S6.3: Cumulative probability distribution of the length of L. lactis FM03-V1 cells during retentostat 1 at various time points. The length of more than 100 cells per time point was determined with ImageJ based on scanning electron microscopy pictures. Blue, red, green, purple and orange represent time point $0,7,21,34$ and 37 days in retentostat cultivation, respectively.

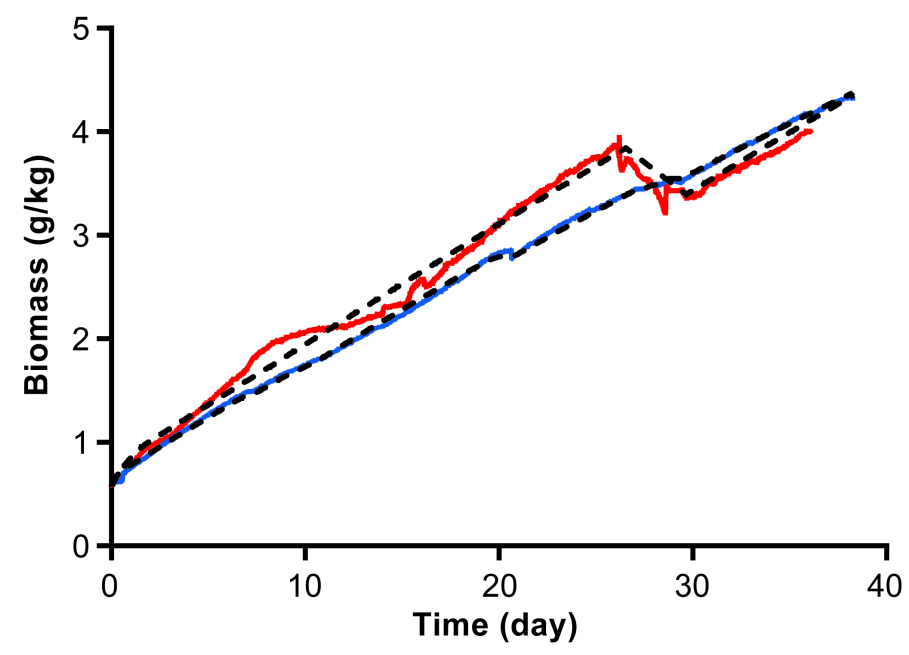

Supplementary Figure S6.4: Growth of L. lactis FM03-V1 during retentostat cultivation. Blue and red solid lines represent the online optical density measurements at $600 \mathrm{~nm}$ of retentostat cultivation 1 and 2, respectively, which are converted to cell dry weight concentrations with a second-order polynomial function (Suppl. Fig. S1). Dashed lines represent predictions of the biomass concentration by model 2. At time zero a chemostat culture in steady state was switched to retentostat mode by insertion of a filter in the effluent line. 

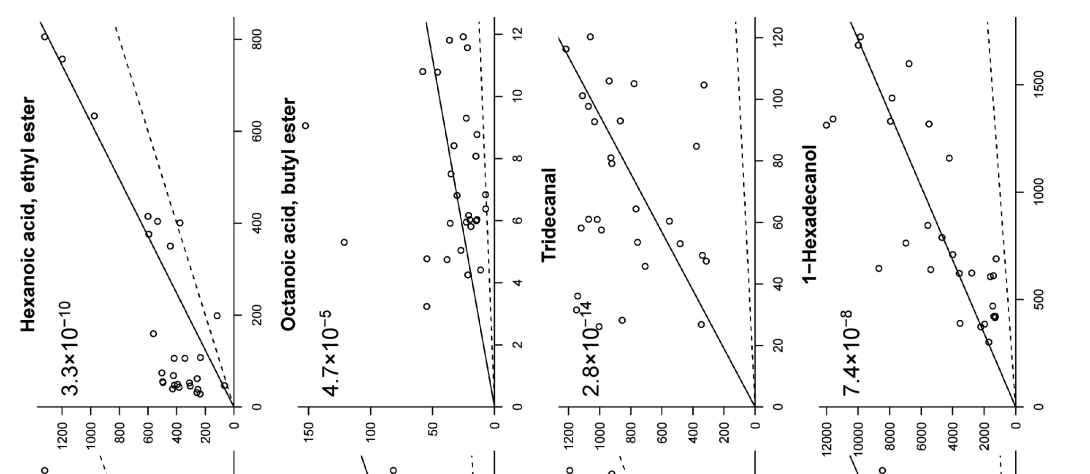

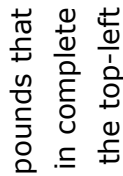

है ญ
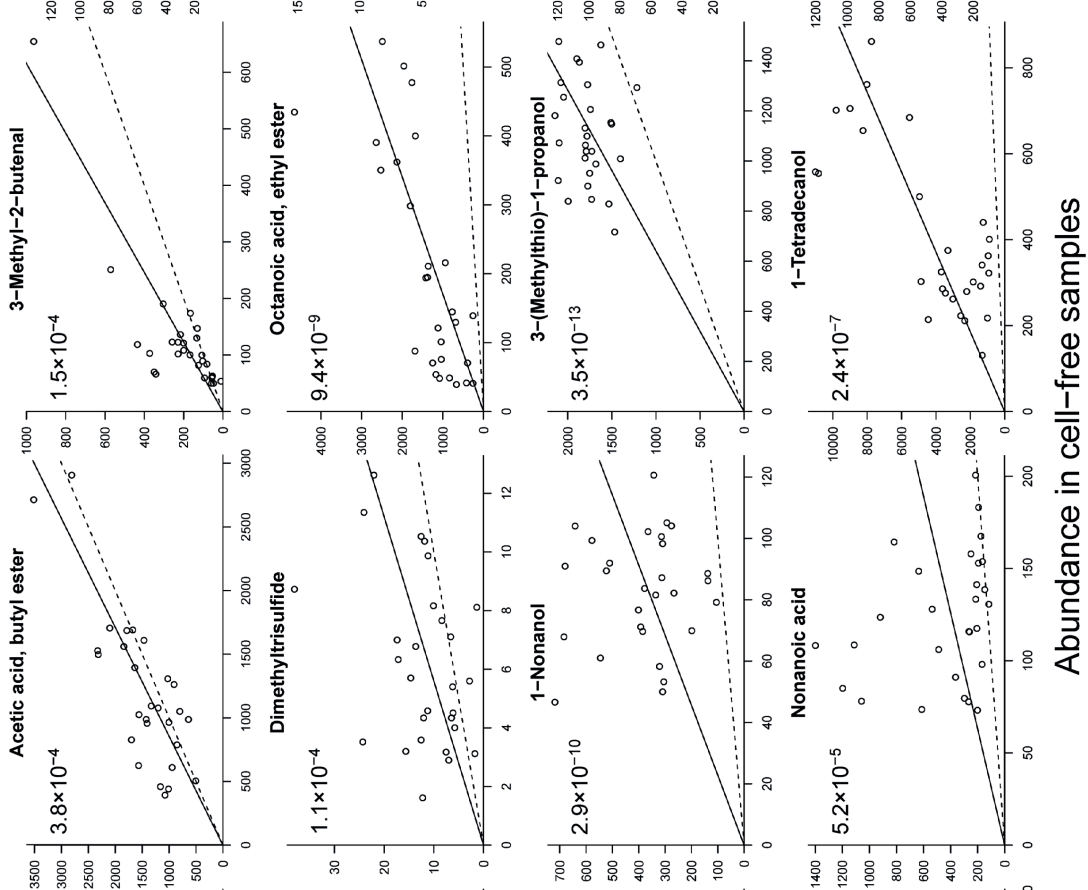

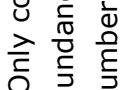

ปิ

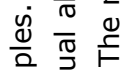

ह

)

妾 仓

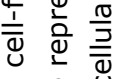

ர $\stackrel{0}{\subseteq} \stackrel{0}{\square}$

ข पृ

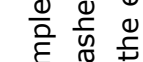

ज 0.5

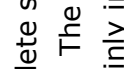

高
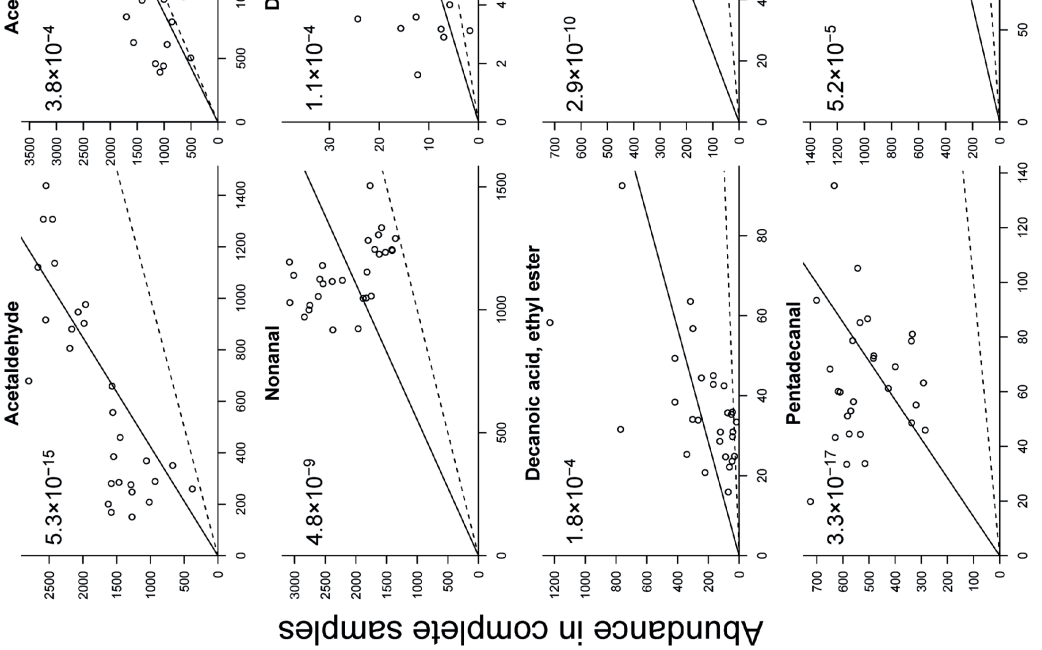

.$\subseteq$
$\mathbb{0}$
$\frac{0}{\frac{1}{0}}$
$\frac{0}{0}$
$\frac{1}{5}$
$\frac{0}{4}$

응 음

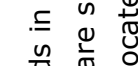

气

है है

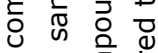

प

\& 응

䒕 है

돈

远婇至

ที $\frac{\pi}{0} \stackrel{0}{\frac{D}{D}}$

ดे

ช

ป⿱艹

i $\frac{1}{2}$ 过

ते

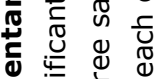

ह sə|dues әाә|dmoว u! әэuepunq $\forall$

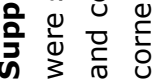



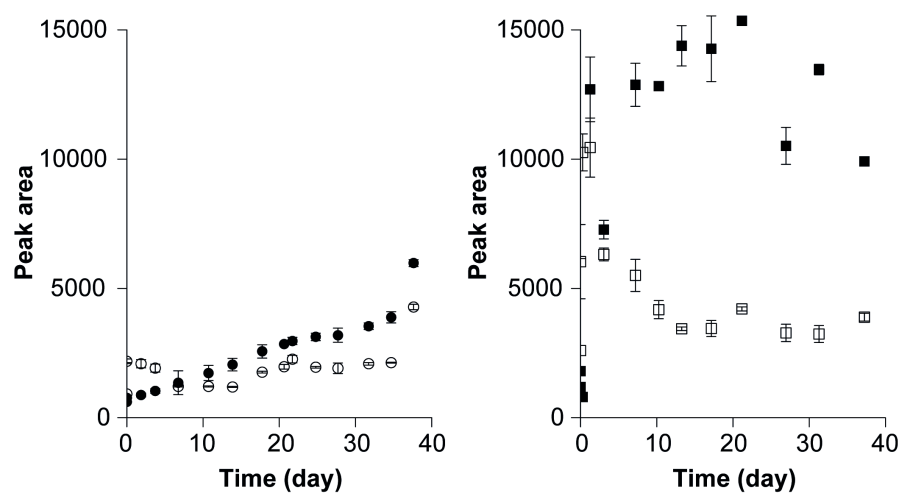

Supplementary Figure S6.6: Abundances of acetoin (open) and 2-butanone (closed) during retentostat cultivation 1 (left, circles) and 2 (right, squares). At time zero a chemostat culture in steady state was switched to retentostat mode by insertion of a filter in the effluent line. Error bars represent the standard deviation of technical duplicates. 


\section{Supplement A: Equivalence of model 1 and 2}

Model 1 (fitted $\mathrm{YX}_{\mathrm{X} / \mathrm{ATP}} \max$ and $\mathrm{m}_{\text {ATP }}$ ) and model 2 (estimated $\mathrm{Y}_{\mathrm{X} / \mathrm{ATP}}$ max from chemostat cultivations and $m_{\text {ATP }}$ linearly dependent on $q_{A T P}$ ) are equivalent to each other if $m_{A T P}$ is smaller than $m_{A T P}$ max as proved by the following derivation.

In both models it was assumed that ATP was used for growth and maintenance processes and the division depends on the maximum biomass yield on ATP ( $\mathrm{Y}_{\mathrm{X} / \mathrm{ATP}}{ }^{\mathrm{max}}$ ) and the maintenance coefficient ( $\left.\mathrm{m}_{\text {ATP }}\right)$. This assumption can be described with equation S6.1.

$$
q_{A T P}=\frac{\mu}{Y_{x / A T P}^{\max }}+m_{A T P}
$$

For model 1 we assumed a constant $m_{\text {ATP }}$ and $Y_{X / A T P}{ }^{\max }$, for model 2 we assumed that the maintenance coefficient was linearly dependent on the biomass specific ATP production rate.

$$
m_{A T P}=a+b \cdot q_{A T P}
$$

Substitution of equation S6.2 in equation S6.1 gives:

$$
q_{A T P}=\frac{\mu}{Y_{x / A T P}^{\max }}+a+b \cdot q_{A T P}
$$

Isolation of $\mathrm{q}_{\mathrm{ATP}}$ in equation $\mathrm{S} 6.3$ gives:

$$
q_{\text {ATP }}=\frac{\mu}{(1-b) \cdot Y_{x / A T P}^{\max }}+\frac{a}{1-b}
$$

Equation S6.4 is equivalent to equation S6.1 because $a, b$ and $\mathrm{Y}_{\mathrm{X} / \mathrm{ATP}}{ }^{\max }$ are all constants. Comparison of equation S6.1, describing model 1, and equation S6.4, describing model 2 , gives the relationship between the $\mathrm{Y}_{\mathrm{X} / \mathrm{ATP}}{ }^{\max }$ and $\mathrm{m}_{\text {ATP }}$ of model 1 and 2.

$$
\begin{gathered}
Y_{x / A T P, 1}^{\max }=(1-b) \cdot Y_{x / A T P, 2}^{\max } \\
m_{A T P, 1}=\frac{a}{1-b}
\end{gathered}
$$




\section{Supplement B: Derivation of equation 6.4 - prediction of biomass concentration in the steady state of a chemostat cultivation}

The change in substrate in the bioreactor depends on the influx of substrate $\left(F_{\text {in }} \cdot C_{s, i n}\right)$, the efflux of residual substrate $\left(F_{\text {out }} \cdot C_{s, \text { out }}\right)$ and the consumption of substrate by the bacteria $\left(q_{s} \cdot C_{x} \cdot V\right)$.

$$
\frac{d\left(V \cdot C_{s}\right)}{d t}=F_{\text {in }} \cdot C_{s, \text { in }}-F_{\text {out }} \cdot C_{s, \text { out }}-q_{s} \cdot C_{x} \cdot V
$$

In the chemostat the volume is kept constant and therefore we assume that the flow of medium to the bioreactor is equal to the efflux of spent medium: $F_{\text {in }}=F_{\text {out }}=F$. Therefore, we can rewrite equation S6.8 to:

$$
\frac{d c_{s}}{d t}=\frac{F}{V} \cdot\left(C_{s, \text { in }}-C_{s, \text { out }}\right)-q_{s} \cdot C_{x}
$$

When the chemostat is in a steady state, there is no change in the substrate concentration $\left(\mathrm{dC}_{\mathrm{s}} / \mathrm{dt}=0\right)$ giving:

$$
0=\frac{F}{V} \cdot\left(C_{s, \text { in }}-C_{s, \text { out }}\right)-q_{s} \cdot C_{x}
$$

Isolating the biomass concentration gives:

$$
C_{x}=\frac{F_{\bar{V}} \cdot\left(C_{s, i n}-C_{s, o u t}\right)}{q_{s}}
$$

The biomass specific substrate consumption rate $q_{s}$ can be expressed in the biomass specific ATP consumption rate and the ATP yield on substrate.

$$
q_{S}=\frac{q_{A T P}}{Y_{A T P / S}}
$$

Substitution of equation $\mathrm{S} 6.12$ in equation S6.11 gives:

$$
C_{x}=\frac{\frac{F}{\bar{V}} \cdot\left(C_{s, \text { in }}-C_{\text {sout }}\right)}{\frac{q_{A T P}}{Y_{A T P / S}}}
$$

ATP is used for growth and maintenance processes and the division depends on the maximum biomass yield on ATP $\left(\mathrm{Y}_{\mathrm{X} / \mathrm{ATP}}{ }^{\max }\right)$ and the maintenance coefficient $\left(m_{\text {ATP }}\right)$.

$$
q_{A T P}=\frac{\mu}{Y_{x / A T P}^{\max }}+m_{A T P}
$$

Substitution of equation $\mathrm{S} 6.14$ in equation $\mathrm{S} 6.13$ followed by some rearrangements gives:

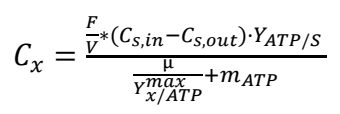


In the steady state of a chemostat, the growth rate $\mu$ equals the dilution rate

$$
\mu=D=\frac{F}{V}
$$

Substitution of equation S6.16 in equation S6.15 gives:

$$
c_{x}=\frac{D \cdot\left(C_{s, i n}-C_{s, o u t}\right) \cdot Y_{A T P / S}}{\frac{D}{Y_{x / A T P}^{m a x}}+m_{A T P}}
$$




\section{References}

Adamberg, K., Kask, S., Laht, T.-M. and Paalme, T. (2003). The effect of temperature and $\mathrm{pH}$ on the growth of lactic acid bacteria: a pH-auxostat study. Int. J. Food Microbiol. 85(1): 171-183.

Barbieri, G., Bolzoni, L., Careri, M., Mangia, A., Parolari, G., Spagnoli, S. and Virgili, R. (1994). Study of the volatile fraction of Parmesan cheese. J. Agric. Food Chem. 42(5): $1170-1176$.

Boender, L. G. M., de Hulster, E. A. F., van Maris, A. J. A., Daran-Lapujade, P. A. S. and Pronk, J. T. (2009). Quantitative physiology of Saccharomyces cerevisiae at nearzero specific growth rates. Appl. Environ. Microbiol. 75(17): 5607-5614.

Boender, L. G. M., van Maris, A. J. A., de Hulster, E. A. F., Almering, M. J. H., van der Klei, I. J., Veenhuis, M., de Winde, J. H., Pronk, J. T. and Daran-Lapujade, P. (2011). Cellular responses of Saccharomyces cerevisiae at near-zero growth rates: transcriptome analysis of anaerobic retentostat cultures. FEMS Yeast Res. 11(8): 603-620.

Brock, T. D. (1971). Microbial growth rates in nature. Bacteriol. Rev. 35(1): 39-58.

Burdock, G. A. (2010). Fenaroli's handbook of flavor ingredients (Sixth ed.). Boca Raton, USA: CRC Press.

Burkhard, L. P., Kuehl, D. W. and Veith, G. D. (1985). Evaluation of reverse phase liquid chromatography/mass spectrometry for estimation of n-octanol/water partition coefficients for organic chemicals. Chemosphere 14(10): 1551-1560.

Cheng, H. (2010). Volatile flavor compounds in yogurt: a review. Crit. Rev. Food Sci. Nutr. 50(10): 938-950.

Collins, Y. F., McSweeney, P. L. H. and Wilkinson, M. G. (2003). Lipolysis and free fatty acid catabolism in cheese: a review of current knowledge. Int. Dairy J. 13(11): 841866.

Condon, S. (1987). Responses of lactic acid bacteria to oxygen. FEMS Microbiol. Lett. 46(3): 269-280.

Curioni, P. M. G. and Bosset, J. O. (2002). Key odorants in various cheese types as determined by gas chromatography-olfactometry. Int. Dairy J. 12(12): 959-984.

Desfossés-Foucault, É., LaPointe, G. and Roy, D. (2013). Dynamics and rRNA transcriptional activity of lactococci and lactobacilli during Cheddar cheese ripening. Int. J. Food Microbiol. 166(1): 117-124.

Dickschat, J. S., Wickel, S., Bolten, C. J., Nawrath, T., Schulz, S. and Wittmann, C. (2010). Pyrazine biosynthesis in Corynebacterium glutamicum. European J. Org. Chem. 2010(14): 2687-2695.

Engels, W. J. M., Dekker, R., de Jong, C., Neeter, R. and Visser, S. (1997). A comparative study of volatile compounds in the water-soluble fraction of various types of ripened cheese. Int. Dairy J. 7(4): 255-263.

Ercan, O., Smid, E. J. and Kleerebezem, M. (2013). Quantitative physiology of Lactococcus lactis at extreme low-growth rates. Environ. Microbiol. 15(8): 2319-2332.

Ercan, O., Bisschops, M. M. M., Overkamp, W., Jorgensen, T. R., Ram, A. F., Smid, E. J., Pronk, J. T., Kuipers, O. P., Daran-Lapujade, P. and Kleerebezem, M. (2015a). Physiological and transcriptional responses of different industrial microbes at nearzero specific growth rates. Appl. Environ. Microbiol. 81(17): 5662-5670.

Ercan, O., Wels, M., Smid, E. J. and Kleerebezem, M. (2015b). Molecular and metabolic adaptations of Lactococcus lactis at near-zero growth rates. Appl. Environ. Microbiol. 81(1): 320-331.

Erkus, O., de Jager, V. C. L., Spus, M., van Alen-Boerrigter, I. J., van Rijswijck, I. M. H., Hazelwood, L., Janssen, P. W. M., van Hijum, S. A. F. T., Kleerebezem, M. and Smid, E. J. (2013). Multifactorial diversity sustains microbial community stability. ISME J 7(11): 2126-2136.

Erkus, O., de Jager, V. C. L., Geene, R. T. C. M., van Alen-Boerrigter, I., Hazelwood, L., van Hijum, S. A. F. T., Kleerebezem, M. and Smid, E. J. (2016). Use of propidium monoazide for selective profiling of viable microbial cells during Gouda cheese ripening. Int. J. Food Microbiol. 228(Supplement C): 1-9. 
Gehrig, R. F. and Knight, S. G. (1961). Formation of 2-heptanone from caprylic acid by spores of various filamentous fungi. Nature 192(4808): 1185-1185.

Goffin, P., van de Bunt, B., Giovane, M., Leveau, J. H. J., Höppener-Ogawa, S., Teusink, B. and Hugenholtz, J. (2010). Understanding the physiology of Lactobacillus plantarum at zero growth. Mol. Syst. Biol. 6(413).

Gogus, F., Ozel, M. Z. and Lewis, A. C. (2006). Analysis of the volatile components of Cheddar cheese by direct thermal desorption-GCXGC-TOF/MS. J. Sep. Science 29(9): 1217-1222.

Hansch, C., Leo, A. and Hoekman, D. (1995). Exploring QSAR: hydrophobic, electronic, and steric constants. Washington, DC: American Chemical Society.

Harvey, R. J. and Collins, E. B. (1961). Role of citritase in acetoin formation by Streptococcus diacetilactis and Leuconostoc citrovorum. J. Bacteriol. 82(6): 954-959.

Hashimoto, N. and Kuroiwa, Y. (1975). Proposed pathways for the formation of volatile aldehydes during storage of bottled beer. Proc. Am. Soc. Brew. Chem. 33: 104111.

Hoehler, T. M. and Jorgensen, B. B. (2013). Microbial life under extreme energy limitation. Nat. Rev. Microbiol. 11(2): 83-94.

Jensen, N. B. S., Melchiorsen, C. R., Jokumsen, K. V. and Villadsen, J. (2001). Metabolic behavior of Lactococcus lactis MG1363 in microaerobic continuous cultivation at a low dilution rate. Appl. Environ. Microbiol. 67(6): 2677-2682.

Keen, A. R., Walker, N. J. and Peberdy, M. F. (1974). The formation of 2-butanone and 2butanol in Cheddar cheese. J. Dairy Res. 41(2): 249-257.

Kempes, C. P., van Bodegom, P. M., Wolpert, D., Libby, E., Amend, J. and Hoehler, T. (2017). Drivers of bacterial maintenance and minimal energy requirements. Front. Microbiol. 8(31).

Koch, A. L. (1971). The adaptive responses of Escherichia coli to a feast and famine existence. Adv. Microb. Physiol. 6: 147-217.

Lahtvee, P.-J., Seiman, A., Arike, L., Adamberg, K. and Vilu, R. (2014). Protein turnover forms one of the highest maintenance costs in Lactococcus lactis. Microbiology 160(7): 1501-1512.

Lever, M. A., Rogers, K. L., Lloyd, K. G., Overmann, J., Schink, B., Thauer, R. K., Hoehler, T. M. and Jorgensen, B. B. (2015). Life under extreme energy limitation: a synthesis of laboratory- and field-based investigations. FEMS Microbiol. Rev. 39(5): 688-728.

Liu, S.-Q., Holland, R. and Crow, V. L. (2004a). Esters and their biosynthesis in fermented dairy products: a review. Int. Dairy J. 14(11): 923-945.

Liu, S.-Q., Baker, K., Bennett, M., Holland, R., Norris, G. and Crow, V. L. (2004b). Characterisation of esterases of Streptococcus thermophilus ST1 and Lactococcus lactis subsp. cremoris B1079 as alcohol acyltransferases. Int. Dairy J. 14(10): 865870.

Majcher, M., Ławrowski, P. and Jeleń, H. (2010). Comparison of original and adulterated Oscypek cheese based on volatile and sensory profiles. Acta Sci. Pol. Technol. Aliment 9(3): 265-275.

Majcher, M. A., Goderska, K., Pikul, J. and Jeleń, H. H. (2011). Changes in volatile, sensory and microbial profiles during preparation of smoked ewe cheese. J. Sci. Food Agric. 91(8): 1416-1423.

Martley, F. G. and Crow, V. L. (1993). Interactions between non-starter microorganisms during cheese manufacture and repening. Int. Dairy J. 3(4): 461-483.

McSweeney, P. L. H. and Fox, P. F. (2004). Metabolism of residual lactose and of lactate and citrate Cheese: Chemistry, Physics and Microbiology (Vol. 1, pp. 361-371): Academic Press.

Meghrous, J., Huot, E., Quittelier, M. and Petitdemange, H. (1992). Regulation of nisin biosynthesis by continuous cultures and by resting cell of Lactococcus lactis subsp. lactis. Res. Microbiol. 143(9): 879-890.

Morita, R. Y. (1993). Bioavailability of energy and the starvation state. In Kjelleberg, S. (Ed.), Starvation in Bacteria (pp. 1-23). Boston, MA: Springer US.

Morita, R. Y. (1997). Bacteria in oligotrophic environments: starvation-survival lifestyle. New York: Chapman \& Hall. 
Nardi, M., Fiez-Vandal, C., Tailliez, P. and Monnet, V. (2002). The EstA esterase is responsible for the main capacity of Lactococcus lactis to synthesize short chain fatty acid esters in vitro. J. Appl. Microbiol. 93(6): 994-1002.

Nierop Groot, M. N. and de Bont, J. A. M. (1998). Conversion of phenylalanine to benzaldehyde initiated by an aminotransferase in Lactobacillus plantarum. Appl. Environ. Microbiol. 64(8): 3009-3013.

Ott, A., Germond, J.-E. and Chaintreau, A. (2000). Vicinal diketone formation in yogurt: $13 \mathrm{C}$ precursors and effect of branched-chain amino acids. J. Agric. Food Chem. 48(3): 724-731.

Otto, R., Sonnenberg, A. S., Veldkamp, H. and Konings, W. N. (1980). Generation of an electrochemical proton gradient in Streptococcus cremoris by lactate efflux. Proc. Natl. Acad. Sci. 77(9): 5502-5506.

Overkamp, W., Ercan, O., Herber, M., van Maris, A. J. A., Kleerebezem, M. and Kuipers, O. P. (2015). Physiological and cell morphology adaptation of Bacillus subtilis at nearzero specific growth rates: a transcriptome analysis. Environ. Microbiol. 17(2): 346363.

Panikov, N. S., Mandalakis, M., Dai, S., Mulcahy, L. R., Fowle, W., Garrett, W. S. and Karger, B. L. (2015). Near-zero growth kinetics of Pseudomonas putida deduced from proteomic analysis. Environ. Microbiol. 17(1): 215-228.

Pirt, S. J. (1965). The maintenance energy of bacteria in growing cultures. Proc. $R$. Soc. Lond. B. Biol. Sci. 163(991): 224-231.

Qian, M. and Reineccius, G. (2002). Identification of aroma compounds in ParmigianoReggiano cheese by gas chromatography/olfactometry. J. Dairy Sci. 85(6): 13621369.

Rebnegger, C., Vos, T., Graf, A. B., Valli, M., Pronk, J. T., Daran-Lapujade, P. and Mattanovich, D. (2016). Pichia pastoris exhibits high viability and a low maintenance energy requirement at near-zero specific growth rates. Appl. Environ. Microbiol. 82(15): 4570-4583.

Ruggirello, M., Dolci, P. and Cocolin, L. (2014). Detection and viability of Lactococcus lactis throughout cheese ripening. PLoS One 9(12): e114280.

Ruggirello, M., Cocolin, L. and Dolci, P. (2016). Fate of Lactococcus lactis starter cultures during late ripening in cheese models. Food Microbiol. 59: 112-118.

Sangster, J. (1989). Octanol-water partition coefficients of simple organic compounds. J. Phys. Chem. Ref. Data 18(3): 1111-1229.

Singh, T. K., Drake, M. A. and Cadwallader, K. R. (2003). Flavor of Cheddar cheese: a chemical and sensory perspective. Compr. Rev. Food Sci. Food Saf. 2(4): 166-189.

Smid, E. J. and Kleerebezem, M. (2014). Production of aroma compounds in lactic fermentations. Annu. Rev. Food Sci. Technol. 5(1): 313-326.

Smit, B. A., Vlieg, J. E. T. V., Engels, W. J. M., Meijer, L., Wouters, J. T. M. and Smit, G. (2005a). Identification, cloning, and characterization of a Lactococcus lactis branched-chain alpha-keto acid decarboxylase involved in flavor formation. Appl. Environ. Microbiol. 71(1): 303-311.

Smit, G., Smit, B. A. and Engels, W. J. M. (2005b). Flavour formation by lactic acid bacteria and biochemical flavour profiling of cheese products. FEMS Microbiol. Rev. 29(3): 591-610.

Snoep, J. L., Teixeira de Mattos, M. J., Starrenburg, M. J. and Hugenholtz, J. (1992). Isolation, characterization, and physiological role of the pyruvate dehydrogenase complex and alpha-acetolactate synthase of Lactococcus lactis subsp. lactis bv. diacetylactis. J. Bacteriol. 174(14): 4838-4841.

Snoep, J. L., de Graef, M. R., Westphal, A. H., de Kok, A., Teixeira de Mattos, M. J. and Neijssel, O. M. (1993). Differences in sensitivity to NADH of purified pyruvate dehydrogenase complexes of Enterococcus faecalis, Lactococcus lactis, Azotobacter vinelandii and Escherichia coli: Implications for their activity in vivo. FEMS Microbiol. Lett. 114(3): 279-283.

Ten Brink, B., Otto, R., Hansen, U. P. and Konings, W. N. (1985). Energy recycling by lactate efflux in growing and nongrowing cells of Streptococcus cremoris. J. Bacteriol. 162(1): 383-390. 
Tewari, Y. B., Miller, M. M., Wasik, S. P. and Martire, D. E. (1982). Aqueous solubility and octanol/water partition coefficient of organic compounds at $25.0^{\circ} \mathrm{C}$. J. Chem. Eng. Data 27(4): 451-454.

Thierry, A., Maillard, M.-B., Richoux, R. and Lortal, S. (2006). Ethyl ester formation is enhanced by ethanol addition in mini Swiss cheese with and without added aropionibacteria. J. Agric. Food Chem. 54(18): 6819-6824.

Thomas, T. D., Ellwood, D. C. and Longyear, V. M. C. (1979). Change from homo- to heterolactic fermentation by Streptococcus lactis resulting from glucose limitation in anaerobic chemostat cultures. J. Bacteriol. 138(1): 109-117.

Thomas, T. D. and Pearce, K. N. (1981). Influence of salt on lactose fermentation and proteolysis in Cheddar cheese. New Zeal J Dairy Sci 16(3): 253-259.

Urbach, G. (1993). Relations between cheese flavour and chemical composition. Int. Dairy J. 3(4): 389-422.

Valvani, S. C., Yalkowsky, S. H. and Roseman, T. J. (1981). Solubility and partitioning IV: Aqueous solubility and octanol-water partition coefficients of liquid nonelectrolytes. J. Pharm. Sci. 70(5): 502-507.

van Leuven, I., van Caelenberg, T. and Dirinck, P. (2008). Aroma characterisation of Goudatype cheeses. Int. Dairy J. 18(8): 790-800.

van Mastrigt, O., Mager, E. E., Jamin, C., Abee, T. and Smid, E. J. (2018). Citrate, low pH and amino acid limitation induce citrate utilisation in Lactococcus lactis biovar diacetylactis. Microb. Biotechnol. 11(2): 369-380.

van Rijswijck, I. M. H., Wolkers - Rooijackers, J. C. M., Abee, T. and Smid, E. J. (2017). Performance of non-conventional yeasts in co-culture with brewers' yeast for steering ethanol and aroma production. Microb. Biotechnol. 10(6): 1591-1602.

van Verseveld, H. W., de Hollander, J. A., Frankena, J., Braster, M., Leeuwerik, F. J. and Stouthamer, A. H. (1986). Modeling of microbial substrate conversion, growth and product formation in a recycling fermentor. Antonie Van Leeuwenhoek 52(4): 325342.

Vos, T., Hakkaart, X. D. V., de Hulster, E. A. F., van Maris, A. J. A., Pronk, J. T. and DaranLapujade, P. (2016). Maintenance-energy requirements and robustness of Saccharomyces cerevisiae at aerobic near-zero specific growth rates. Microb. Cell Fact. 15: 111.

Yamagami, C., Takao, N. and Fujita, T. (1991). Hydrophobicity parameter of diazines. II: Analysis and prediction of partition coefficients of disubstituted pyrazines. J. Pharm. Sci. 80(8): 772-777.

Yvon, M. and Rijnen, L. (2001). Cheese flavour formation by amino acid catabolism. Int. Dairy J. 11(4): 185-201. 


\section{Chapter 7}

\section{Aroma formation in retentostat co-cultures of Lactococcus lactis and Leuconostoc mesenteroides}

Oscar van Mastrigt, Reinier A. Egas, Tjakko Abee \& Eddy J. Smid 


\begin{abstract}
Lactococcus lactis biovar diacetylactis and Leuconostoc mesenteroides are considered to be the main aroma producers in Dutch-type cheeses. Both species of lactic acid bacteria were grown in retentostat mono- and co-cultures to investigate their interaction at near-zero growth rates and to determine if co-cultivation enhances the aroma complexity compared to single species performance. While the growth rates of both species decreased to less than $0.001 \mathrm{~h}^{-1}$, the viability of the cells remained above $80 \%$. However, a large fraction lost the ability to grow on agar plates indicating that cells were viable but not culturable (VBNC). Compared to LC. mesenteroides, L. lactis reached a 3.4-fold higher biomass concentration caused by i) a higher ATP yield on substrate, ii) a higher biomass yield on ATP and iii) a lower maintenance requirement. Dynamic models estimated that the maintenance coefficient $\left(m_{\text {ATP }}\right)$ of both species decreased approximately 7-fold at near-zero growth rates compared to high growth rates. These dynamic models were extended to predict accumulation of both species in retentostat co-cultures by assuming equal uptake kinetics of both species for lactose and citrate. This resulted in good prediction of the biomass accumulation with $L$. lactis dominating the co-culture (ratio of 100:1) in a similar way as observed in ripened cheese. In total, 47 aroma compounds were produced by both species of which 17 and 5 were found to be specific for $L$. lactis and $L C$. mesenteroides, respectively. Despite its low abundance $(\sim 1 \%), L C$. mesenteroides contributed to aroma production in co-cultures as indicated by the presence of 2 out of 5 specific LC. mesenteroides compounds. Co-cultures lacked additional unique aroma compounds indicating that a similar aroma complexity could be obtained by mixing retentostat mono-cultures.
\end{abstract}




\section{Introduction}

Mesophilic mixed DL-type starter cultures used for the production of Dutch-type cheeses consist of the homofermentative Lactococcus lactis subsp. cremoris, Lactococcus lactis subsp. lactis, Lactococcus lactis biovar diacetylactis and the heterofermentative Leuconostoc spp. (Frantzen et al., 2017). L. lactis subsp. cremoris and $L$. lactis subsp. lactis are considered to be the main acid producers that dominate in the early stages of cheese making and quickly decline during cheese ripening (Erkus et al., 2013). In contrast, L. lactis subsp. lactis biovar diacetylactis and Leuconostoc spp. become dominant during cheese ripening due to better survival and are the main aroma and texture producers. The aroma producers generally account for $1-10 \%$ of the starter culture population (Cogan and Jordan, 1994, Erkus et al., 2013). Both species are able to metabolise citrate and produce the important flavours diacetyl and acetoin as well as $\mathrm{CO}_{2}$, which is important for eye formation (Hugenholtz, 1993). Other important aroma compounds in cheese result from the metabolism of fatty acids and amino acids of which aromatic, sulphur-containing and branched-chain amino acids are considered to be most important (Yvon and Rijnen, 2001).

Recently, it was demonstrated that aroma formation by L. lactis subsp. lactis biovar diacetylactis was greatly affected by slow growth of the bacteria using retentostat cultivation, thereby better resembling aroma formation during cheese ripening (van Mastrigt et al., 2018a). Retentostat cultivation is a modification of chemostat cultivation in which a filter is connected to the effluent line thereby recycling the biomass to the bioreactor (Boender et al., 2009). This leads to accumulation of the biomass in time and a severe reduction in growth rate that approaches zero. Growth rates below $0.001 \mathrm{~h}^{-1}$ were obtained with the dairy $L$. lactis biovar diacetylactis FM03-V1 (van Mastrigt et al., 2018b). In addition to changes in aroma formation, the number of viable but non-culturable cells increased and the maintenance requirements decreased most likely due to a decrease in the energy spent on protein turnover (van Mastrigt et al., 2018b).

While studying aroma formation by lactic acid bacteria using retentostat cultivation, it has to be taken into account that starter cultures often consist of multiple strains and species. Metabolic complementation of strains could result in new compounds that cannot be produced by the single strains, thereby increasing the aroma complexity. Such complementation has been suggested for L. lactis and $L c$. mesenteroides for the conversion of glutamate into succinate (Erkus et al., 2013) 
To study such interactions under relevant conditions for cheese ripening, i.e. slow growth, L. lactis biovar diacetylactis FM03-V1 and Lc. mesenteroides FM06 were grown in retentostat mono- and co-cultures and the production of aroma compounds was compared. Moreover, dynamic models were developed to describe growth in retentostat mono-cultures and these models were used to predict growth of both species in retentostat co-cultures to identify growth stimulating or inhibiting interactions between the species.

\section{Materials and methods}

\section{Strain and media}

In this study Lactococcus lactis subsp. lactis biovar diacetylactis FM03-V1 and Leuconostoc mesenteroides FM06 were used, which were both isolated from 10week-old Sams $\varnothing$ cheese (van Mastrigt et al., 2017). For chemostat and retentostat cultivation, the bacteria were streaked from a $-80^{\circ} \mathrm{C}$ stock onto $\mathrm{M} 17$ agar plates supplemented with $0.5 \%(\mathrm{w} / \mathrm{v})$ lactose (LM17) and incubated for 2 days at $30^{\circ} \mathrm{C}$. A single colony was transferred to $10 \mathrm{ml}$ lactose-containing chemically defined medium (van Mastrigt et al., 2018c) and grown overnight at $30^{\circ} \mathrm{C}$. The bioreactors were inoculated with the overnight culture at a $1 \%(\mathrm{v} / \mathrm{v})$ inoculated level. The lactose-limited chemically defined medium used for the chemostat and retentostat cultivations contained $0.5 \%(\mathrm{w} / \mathrm{w})$ lactose, $10 \mathrm{mM}\left(\mathrm{NH}_{4}\right)_{3}$ citrate and $1 \%(\mathrm{w} / \mathrm{w})$ Bacto tryptone (van Mastrigt et al., 2018c) and was prepared in $20 \mathrm{~L}$ batches.

\section{Retentostat cultivation}

Retentostat cultivations were performed in bioreactors with a working volume of $1 \mathrm{~L}$ (Infors HT, Switzerland) as previously described (van Mastrigt et al., 2018b). The stirring speed was set at $400 \mathrm{rpm}$, the temperature was maintained at $30^{\circ} \mathrm{C}$ and the $\mathrm{pH}$ was controlled at 5.5 by automatic addition of $5 \mathrm{M} \mathrm{NaOH}$. Anaerobic conditions were maintained by flushing the headspace with nitrogen gas at 0.1 $\mathrm{L} / \mathrm{min}$. The optical density at $600 \mathrm{~nm}$ was continuously measured by an internal probe (Trucell 2, Finesse, USA). Retentostat cultivations were initiated by connecting a sterilisable polyethersulfone cross-flow filter (Spectrum laboratories, USA) to the effluent line after reaching a steady state in the chemostat cultivations at a dilution rate of $0.025 \mathrm{~h}^{-1}$. A steady state was considered to be achieved after a minimum of five volume changes at which the optical density remained constant. In case of co-cultivation, $L$. lactis and Lc. mesenteroides were pre-grown separately in $0.5 \mathrm{~L}$ chemostat cultures at a dilution rate of $0.025 \mathrm{~h}^{-1}$ and, after achieving a steady state, both cultures were transferred to a $1 \mathrm{~L}$ bioreactor 
equipped with a cross-flow filter to initiate the retentostat cultivation. To determine the maximum biomass yield on ATP ( $\left.\mathrm{Y}_{\mathrm{X} / \mathrm{ATP}}{ }^{\max }\right)$ and the maintenance coefficient ( $m_{\text {ATP }}$ ) of L. lactis FM03-V1 and Lc. mesenteroides FM06, the bacteria were grown in chemostat cultures at dilution rates between 0.025 and $0.4 \mathrm{~h}^{-1}$.

\section{Biomass determination}

To monitor the biomass accumulation in the chemostat and retentostat cultures, the cell dry weight concentration was measured as previously described (van Mastrigt et al., 2018c). Briefly, culture samples of 3 to $4 \mathrm{ml}$ were taken directly from the bioreactor and passed through pre-weighted $0.2 \mu \mathrm{m}$ membrane filters (Pall Corporation, USA) by a vacuum filtration unit. The filters were dried at $80^{\circ} \mathrm{C}$ for at least 2 days and weighted again to determine the dry weight of cell biomass.

\section{Cell counts}

To determine the total number of cells in the continuous cultures, samples were diluted 1000 times with physiological salt solution (PPS; Tritium Microbiologie, The Netherlands) and $25 \mu \mathrm{l}$ of this diluted suspension was added to a Bürker-Türk counting chamber (CellVision technologies, Netherlands). Cells were counted at 1000 times $(10 \times 100)$ magnification using a phase contrast microscope (Olympus, Japan).

\section{Plate counts}

To quantify the number of colony forming units (CFUs), appropriate dilutions of the cultures in PPS were plated on agar plates and the number of CFUs were counted after incubation for 3 days at $30^{\circ} \mathrm{C}$. For mono-cultures, MRS and LM17 plates were used to quantify $L C$. mesenteroides and $L$. lactis, respectively. For cocultures, MRS supplemented with $30 \mathrm{mg} / \mathrm{L}$ vancomycin, LM17 and MRS plates were used to quantify $L C$. mesenteroides, L. lactis and both bacteria, respectively.

\section{Cell viability}

The viability of the cultures was determined by LIVE/DEAD Baclight Bacterial Viability kit (Molecular Probes Europe, Netherlands). Bacteria were strained with $3.34 \mu \mathrm{M}$ green fluorescent SYTO 9 and $20 \mu \mathrm{M}$ red fluorescent propidium iodide for 10 minutes at room temperature in the dark. Green and red fluorescent cells were visualised using a fluorescence microscope with an excitation light source (Excelitas, USA) at 630 or 1000 times magnification. Pictures were taken with a 
camera attached to the microscope and the number of green and red cells was counted manually.

\section{Determination of viable $L$. lactis and $L c$. mesenteroides in co-cultures}

The ratio of viable cells of $L$. lactis and $L c$. mesenteroides in co-cultures was determined in three steps: i) propidium monoazide (PMA) treatment, ii) DNA extraction and iii) quantitative PCR (qPCR) analysis. One $\mathrm{ml}$ culture with an optical density at $600 \mathrm{~nm}$ of 0.1 was centrifuged $(17000 \times g$ for 5 minutes) and washed with $500 \mu \mathrm{l}$ phosphate buffered saline (PBS) and resuspended in $1 \mathrm{ml}$ PBS. Subsequently, $2.5 \mu \mathrm{l} 20 \mathrm{mM}$ PMA was added and the cell suspension was incubated for 5 minutes in the dark at room temperature with mixing every minute. The samples were exposed to light for 5 minutes with a PMA-Lite ${ }^{\mathrm{TM}}$ led photolysis device (Biotium, USA) to activate the PMA. DNA was extracted using the DNeasy Blood and Tissue kit (Qiagen, Germany). The sample was centrifuged $(17000 \times g$ for 5 minutes), cells were resuspended in $0.5 \mathrm{ml}$ lysis buffer $(20 \mathrm{mM}$ Tris- $\mathrm{HCl}, 2$ $\mathrm{mM}$ EDTA, $1.2 \%(\mathrm{w} / \mathrm{v})$ Triton X-100, $1 \mathrm{mg} / \mathrm{ml}$ lysozyme, $50 \mathrm{U}$ mutanolysin, $\mathrm{pH}$ 8.0) and the mixture was incubated for 1 hour at $37^{\circ} \mathrm{C}$. Subsequently, $30 \mu \mathrm{l}$ proteinase $\mathrm{K}$ and $250 \mu \mathrm{l} \mathrm{AL}$ buffer were added and the sample was incubated for 1 hour at $56^{\circ} \mathrm{C}$. Subsequently, $250 \mu \mathrm{l}$ absolute ethanol was added and the released DNA was purified using a spin column provided by the kit. $500 \mu$ solution was transferred to the spin column, left for 5 minutes in the column, centrifuged for 1 minute at $6000 \times g$ and washed subsequently with $500 \mu \mathrm{l} \mathrm{AW1}$ and $500 \mu \mathrm{lWW}$. The remaining traces of ethanol were removed by centrifugation for 4 minutes at $17000 \times g$. The DNA was eluted with three times $50 \mu \mathrm{l}$ AE buffer after incubation for 10 minutes at room temperature. Purified DNA was stored at $-20^{\circ} \mathrm{C}$ until analysis with qPCR. DNA extraction efficiencies of $L$. lactis and $L c$. mesenteroides were compared by extraction of DNA in triplicate from chemostat mono-cultures at a dilution rate of $0.025 \mathrm{~h}^{-1}$ according to the above protocol. This showed that extraction efficiencies of both species were not significantly different (t-test assuming equal variances, two-sided, degrees of freedom $=4, t$ value $=-1.26, P$ value $=0.27)$. DNA of $L$. lactis and $L C$. mesenteroides were quantified with qPCR (Bio-Rad Thermal cycler CFX96-Real-Time system) targeting the single-copy gene glyA and using TaqMan probes. Sequences of the used primers and probes are given in Table 7.1. The PCRs took place in $20 \mu$ reaction mixtures containing 0.2 mM dNTPs, $0.5 \mu \mathrm{M}$ forward primer, $0.5 \mu \mathrm{M}$ reverse primer, $0.31 \mu \mathrm{M}$ TaqMan probe, $2 \mu \mathrm{l}$ 10x DreamTaq buffer with $\mathrm{MgCl}_{2}$ (Thermo Scientific, USA), 0.8 U DreamTaq polymerase and $2 \mu \mathrm{l}$ purified DNA. PCR amplification was initiated with 5 minutes at $95^{\circ} \mathrm{C}$ followed by 39 cycles of 10 seconds at $95^{\circ} \mathrm{C}$ and 20 seconds at $59^{\circ} \mathrm{C}$. Fluorescence was measured at the end of each cycle. A threshold of 400 relative 
fluorescence unit (RFU) was used to determine the threshold cycle $\left(C_{t}\right)$. The efficiency of the PCRs of both species was not significantly different from 2 (mean \pm standard error was $2.04 \pm 0.10$ and $2.03 \pm 0.03$ for $L$. lactis and $L C$. mesenteroides, respectively.). Therefore, an efficiency of 2 was used to calculated the ratio of L.lactis:LC. mesenteroides in the co-cultures using equation 7.1:

$$
\text { L.lactis : Lc.mesenteroides }=2^{C t_{\text {Lc.mesenteroides }}-C t_{\text {Llactis }}}
$$

To calculate the viability of $L C$. mesenteroides during the co-cultures, qPCR was also performed using DNA without a PMA treatment, which showed that the viability was at least $33 \%$ throughout the co-cultures.

\section{Analysis of extracellular metabolites}

Lactose, citrate, lactate, acetate, ethanol, formate, pyruvate and acetoin were quantified by High Performance Liquid Chromatography (HPLC) as previously described (van Mastrigt et al., 2018c). Briefly, we used an Ultimate 3000 instrument (Dionex, Germany) equipped with an Aminex HPX-87H column (BioRad, USA) with pre-column at $40^{\circ} \mathrm{C}$ and $5 \mathrm{mM} \mathrm{H}_{2} \mathrm{SO}_{4}$ was used as mobile phase at $0.6 \mathrm{ml} / \mathrm{min}$. Compounds were detected with a Shodex RI-101 refractive index detector (Showa DendoKK, Japan)

\section{Volatile organic compounds analysis}

Volatile organic compounds (VOCs) were analysed by headspace solid phase microextraction gas chromatography mass spectrometry (HS SPME GC-MS) as previously described (van Mastrigt et al., 2018b). $100 \mu$ sample was incubated for 10 minutes at $60^{\circ} \mathrm{C}$ and VOCs were extracted for 20 minutes at $60^{\circ} \mathrm{C}$ using a SPME fiber ( $85 \mathrm{~mm}$ CAR/PDMS, Supelco, USA). Compounds were desorbed for 10 minutes on a Stabilwax ${ }^{\circledR}$-DA-Crossband ${ }^{\circledR}$ - Carbowax ${ }^{\circledR}$-polyethylene-glycol column (30 m length, $0.25 \mathrm{~mm}$ ID, $0.5 \mu \mathrm{m}$ df). PTV Splitless mode was used at $250^{\circ} \mathrm{C}$ for 5 minutes. Helium was used as carrier gas at a constant flow of $1.5 \mathrm{ml} / \mathrm{min}$. The temperature of the $\mathrm{GC}$ oven was initially $40^{\circ} \mathrm{C}$ and after 2 minutes the temperature was raised to $240^{\circ} \mathrm{C}$ at a rate of $10^{\circ} \mathrm{C} / \mathrm{min}$ and kept at $240^{\circ} \mathrm{C}$ for 5 minutes. Mass spectral data was collected over a range of $\mathrm{m} / \mathrm{z} 33-250$ in full scan mode with 3.0030 scans/second. Aroma profiles were analysed using Chromeleon 4.2 software. Peaks were integrated using the ICIS algorithm and the mass spectral profiles were matched with the NIST main library for identification. One peak was used for quantification (in general the highest $\mathrm{m} / \mathrm{z}$ peak per compound) and 1 or 2 peaks were used for confirmation. Compounds related to the medium (e.g. 1-butanol or esters of 1-butanol) were excluded from all analyses. 


\section{Principal component analysis}

Principal component analysis (PCA) of the aroma profiles in mono- and co-cultures were performed in $\mathrm{R}$ (version 3.1.3) using scaled peak areas (mean peak area of every compound is 0 and standard deviation is 1 ).

\section{Mathematical modelling of biomass accumulation}

\section{Mono-cultures}

Biomass accumulation in the retentostat mono-cultures were modelled according to van Mastrigt et al. (2018b) using equation 7.2.

$$
C_{X}(t)=\left(C_{X, 0}-\frac{D \cdot\left(C_{S, i n}-C_{S}\right) \cdot Y_{A T P / S}}{m_{A T P}}\right) \cdot e^{-m_{A T P} \cdot Y_{x / A T P}^{\max } \cdot t}+\frac{D \cdot\left(C_{S, i n}-C_{S}\right) \cdot Y_{A T P / S}}{m_{A T P}}
$$

in which $C_{x}$ is the biomass concentration $(g D W / k g), C_{x, 0}$ is the initial biomass concentration $(\mathrm{gDW} / \mathrm{kg}), \mathrm{D}$ is the dilution rate $\left(\mathrm{h}^{-1}\right), \mathrm{C}_{\mathrm{s}, \mathrm{in}}$ is the substrate concentration in the medium $\left(14.6 \mathrm{mmol} / \mathrm{kg}\right.$ lactose and $10 \mathrm{mmol} / \mathrm{kg}$ citrate), $\mathrm{C}_{\mathrm{S}}$ is the substrate concentration in the effluent, $\mathrm{Y}_{\text {ATP/S }}$ is the ATP yield on substrate (mol ATP/CmolS), $m_{\text {ATP }}$ is the maintenance coefficient (mol ATP.gDW ${ }^{-1} \cdot \mathrm{h}^{-1}$ ),

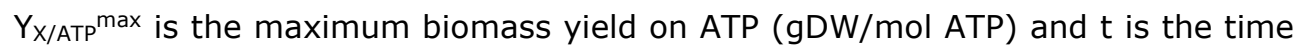
(h). The $Y_{\text {ATP/S }}$ was calculated based on the measured metabolite production. The $Y_{X / A T P}{ }^{\max }$ was estimated in chemostat cultures with a dilution rate between 0.025 and $0.4 \mathrm{~h}^{-1}$. The $\mathrm{m}_{\text {ATP }}$ was assumed to be linearly dependent on the biomass specific ATP production rate ( $q_{A T P}$ ) with a maximum $m_{A T P} \max$ (Eq. 7.3).

$$
m_{A T P}=a+b \cdot q_{A T P} \text { with } m_{a t p} \leq m_{A T P}^{\max }
$$

Input data for the modelling were online optical density measurements, which were converted to cell dry weight concentrations using a second-order polynomial relation. The variable parameters ( $a, b$ and $m_{A T P} \max$ in equation 7.3 ) were optimised by minimising the sum of squared errors between the model and the data in 10 minute intervals using the solver add-in of Microsoft Excel.

\section{Co-cultures}

The optimised variable parameters of the mono-cultures were used in a co-culture model to predict the biomass accumulation of $L$. lactis and Lc. mesenteroides in the co-cultures. The co-culture model followed the mono-culture model with the addition that we assumed $\mathrm{i}$ ) equal uptake kinetics of both species for lactose and citrate, in other words substrate consumption was divided over both species based on the biomass concentration and ii) no lysis of the species even when $q_{\text {ATP }}<$ $\mathrm{m}_{\text {ATP. }}$. 


\section{Results}

\section{Biomass accumulation in retentostat mono-cultures}

Lactococcus lactis FM03-V1 and Leuconostoc mesenteroides FM06 were grown anaerobically in four independent retentostat mono-cultures on a chemically defined medium containing both lactose and citrate. The biomass concentration was measured every 3 to 4 days and the growth rates were estimated. Moreover, we quantified the number of colony forming units by plating on LM17 or MRS and the culture viability by live/dead staining with SYTO 9 and propidium iodide. The retentostat cultures of $L$. lactis has previously been described (van Mastrigt et al., 2018b). For L. lactis and Lc. mesenteroides the biomass concentration increased to 4.2 and $1.2 \mathrm{gDW} / \mathrm{kg}$, respectively, which corresponded to an 8- and 6-fold increase (Fig. 7.1A). The higher biomass concentration of L. lactis indicates a lower maintenance requirement, i.e. less substrate required to maintain cells and/or a higher biomass yield on substrate. The growth rate of both species gradually decreased to less than $0.001 \mathrm{~h}^{-1}$, corresponding to doubling times of more than a month (Fig. 7.1B). The viability remained above $80 \%$ throughout all cultivations (Fig. 7.1C). Despite the observed increases in biomass, the plate counts hardly increased during the cultivations (Fig. 7.1D), indicating that a large fraction of the cells became viable but not culturable (VBNC). This was confirmed for Lc. mesenteroides by microscopic cell counting (Fig. 7.1E), which showed a 6fold increase similar to the observed increase in biomass concentration. This indicates that both species had very similar physiological responses towards nearzero growth rates.

\section{Central metabolism}

The higher biomass concentration of $L$. lactis could be explained by the different metabolic pathways for lactose utilisation of both species. The homofermentative L. lactis uses the Embden-Meyerhof-Parnas (EMP) pathway producing 4 to $6 \mathrm{~mol}$ ATP per mol lactose when it produces lactate (homolactic fermentation) or formate, acetate and ethanol (mixed-acid fermentation), respectively. The heterofermentative $L c$. mesenteroides uses the phosphoketolase pathway producing only 2 mol ATP per mol lactose and equimolar amounts of lactate and ethanol. By using the co-substrate citrate as alternative electron acceptor (Schmitt et al., 1990), 1 extra ATP can be produced per mol citrate by Lc. mesenteroides via acetate kinase. The main substrates (lactose and citrate) and products (lactate, ethanol, acetate, formate, acetoin, pyruvate) were quantified with HPLC to determine the amount of ATP that they theoretically gained from lactose and citrate metabolism (Fig. 7.2). 
A

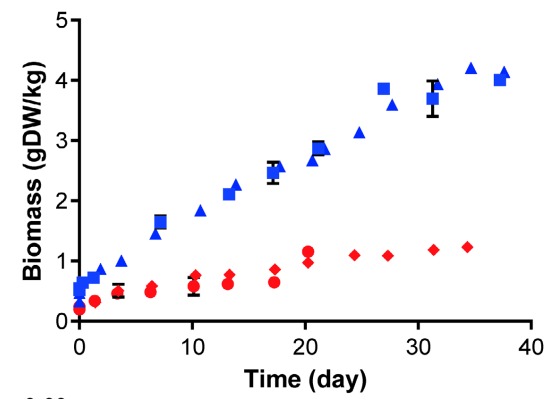

$\mathrm{B}$
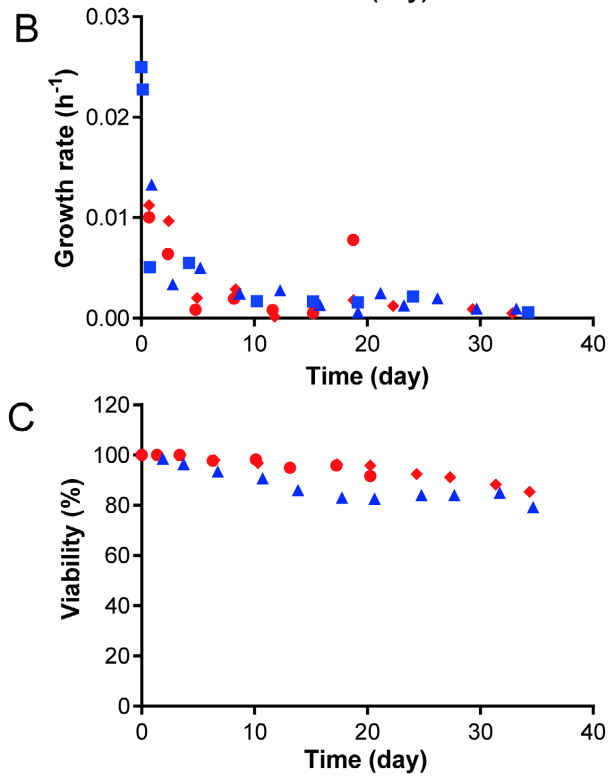
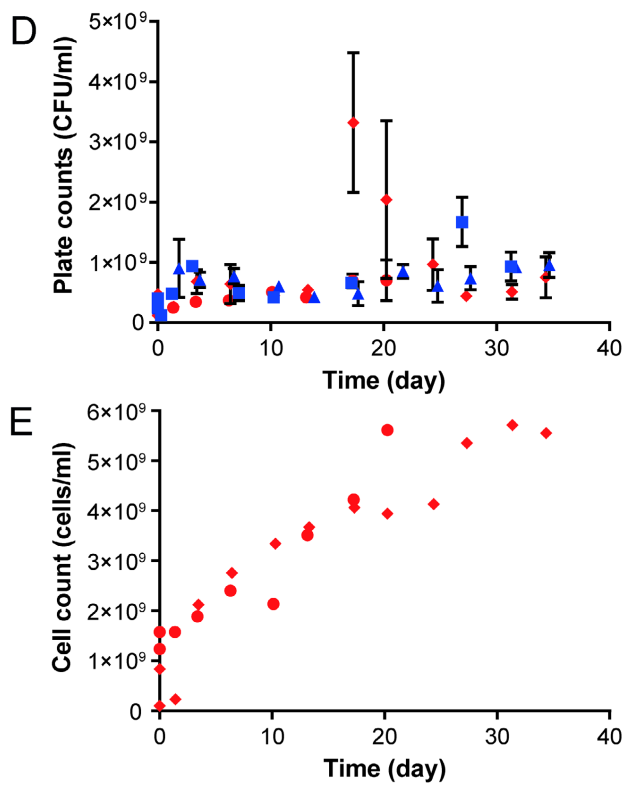

Figure 7.1: Growth of L. lactis FM03-V1 (blue) and LC. mesenteroides FM06 (red) in retentostat mono-cultures. Squares, triangles, diamonds and circles represent four independent retentostat cultures. At time 0 a chemostat culture in steady state was switched to retentostat mode by insertion of a filter in the effluent line. A: Measured biomass concentrations. Data points represent the mean \pm standard deviation of duplicate samples. B: Calculated growth rates based on the biomass concentrations. C: Viability determined using SYTO 9 and propidium iodide as fluorescent markers and fluorescent microscopy for visualisation. D: Plates counts of $L$. lactis and LC. mesenteroides on LM17 and MRS, respectively. Data points represent the mean \pm standard deviation. E: Microscopic cells counts using a counting chamber.

L. lactis mainly produced lactate, acetate, ethanol and formate via a combination of homolactic and mixed-acid fermentation (ratio of approximately 60:40), which resulted in approximately $0.33 \mathrm{~mol}$ ATP per Cmol substrate (lactose and citrate). Lc. mesenteroides produced mainly lactate, ethanol and acetate resulting in a ATP yield of $0.18 \mathrm{~mol}$ ATP per Cmol substrate (lactose and citrate). This confirmed our hypothesis $L$. lactis metabolised the lactose and citrate more efficiently resulting 


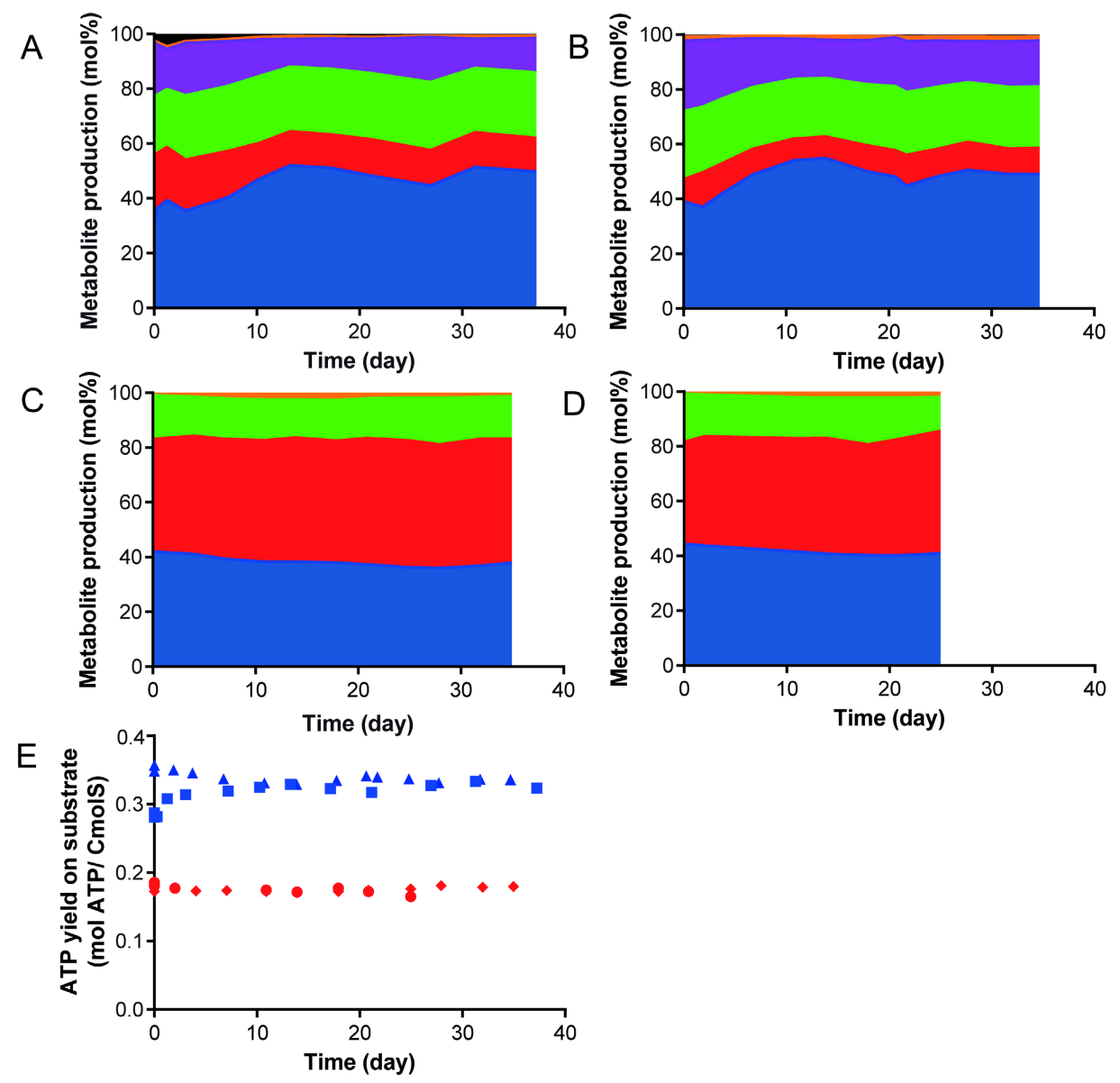

Figure 7.2: Metabolite production of $L$. lactis ( $A$ and $B$ ) and $L C$. mesenteroides ( $C$ and $D$ ) in independent retentostat mono-cultures and the calculated ATP yield on substrate (E). At time 0 a chemostat culture in steady state was switched to retentostat mode by insertion of a filter in the effluent line. Electron-balances, which were calculated using the degree of reduction of the main substrate and products, were always between 94 and $106 \%$. Metabolite production was normalised to $100 \%$. A, B, C, D: Blue, red, green, purple, orange and black represent lactate, ethanol, acetate, formate, pyruvate and acetoin, respectively. E: The ATP yield on substrate ( $\mathrm{Y}_{\mathrm{ATP} / \mathrm{S}}$ ) (lactose and citrate) of L. lactis FM03-V1 (blue) and LC. mesenteroides FM06 (red) was calculated based on the metabolite production profiles and the known metabolism of $L$. lactis and $L c$. mesenteroides. We assumed that 1 ATP was produced by uptake and conversion of citrate into pyruvate. Symbols represent independent retentostat cultivations.

in higher biomass concentrations in retentostat cultures. Interestingly, acetate production by $L c$. mesenteroides via acetate kinase was very limited (on average $0.04 \mathrm{mmol} / \mathrm{h}$ ) despite use of citrate. Possibly, acetate kinase was not very active due to the anaerobic conditions. 


\section{Modelling of retentostat mono-cultures}

Biomass accumulation in retentostat cultures was modelled according to a model described previously (van Mastrigt et al., 2018b), assuming i) a constant maximum biomass yield on ATP ( $\mathrm{Y}_{\mathrm{X} / \mathrm{ATP}}{ }^{\mathrm{max}}$ ) and ii) a maintenance coefficient ( $\mathrm{m}_{\text {ATP }}$ ) that was linearly dependent on the biomass specific ATP production rate (qATP). The $\mathrm{Y}_{\mathrm{X} / \mathrm{ATP}}{ }^{\max }$ and $\mathrm{m}_{\text {ATP }}$ of $L$. lactis and $L C$. mesenteroides were determined in chemostat cultures at dilution rates between 0.025 and $0.4 \mathrm{~h}^{-1}$ (Fig. 7.3).

L. lactis had a higher $\mathrm{Y}_{\mathrm{X} / \mathrm{ATP}}{ }^{\max }$ than $L c$. mesenteroides (mean $\pm \mathrm{SE} ; 15.94 \pm 0.42$ and $9.52 \pm 0.73 \mathrm{gDW} / \mathrm{mol}$ ATP) and a lower $\mathrm{m}_{\text {ATP }}$ (mean $\pm \mathrm{SE} ; 2.43 \pm 0.35$ and $5.32 \pm 0.93 \mathrm{mmol}$ ATP.gDW ${ }^{-1} \cdot \mathrm{h}^{-1}$ ) contributing to the higher biomass concentrations in the retentostat cultures. The determined maximum biomass yields on ATP were included in the model and the biomass accumulation was fitted with the model (as described in the materials and methods).

Good fits were obtained for retentostat cultures of both $L$. lactis and $L C$. mesenteroides (root mean square error of $0.098,0.028,0.020$ and 0.094 $\mathrm{gDW} / \mathrm{kg}$, respectively) (Fig. 7.4). The model predicted that the maintenance coefficient of $L$. lactis and $L C$. mesenteroides decreased approximately 7 -fold at near-zero growth rates to 0.36 and $0.79 \mathrm{mmol}$ ATP.gDW. ${ }^{-1} \cdot \mathrm{h}^{-1}$, respectively (Suppl. Fig. S7.1).

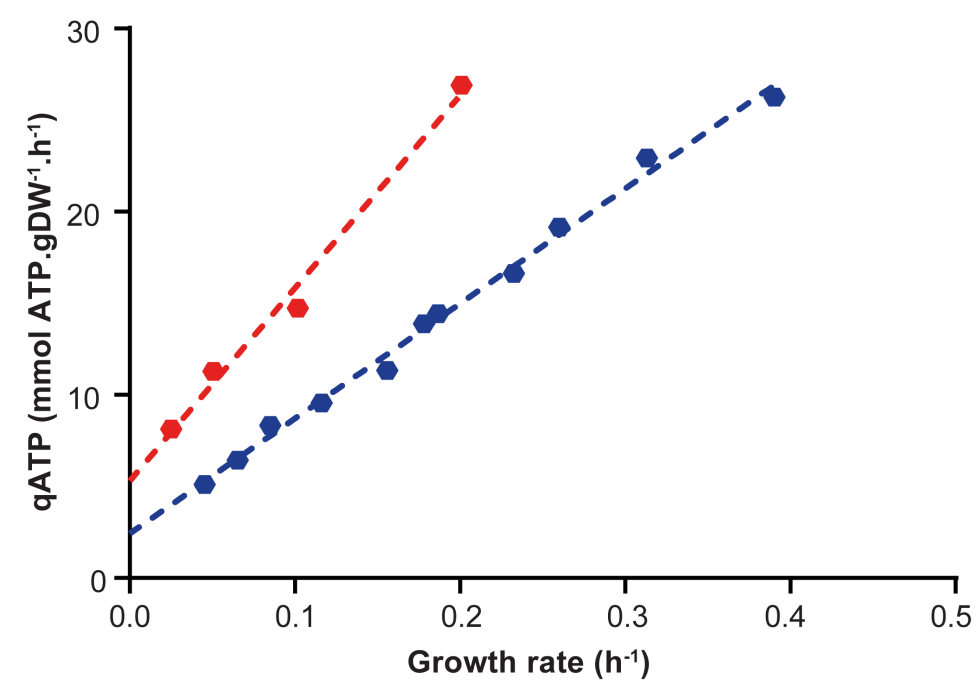

Figure 7.3: Relation between the specific growth rate and the biomass specific ATP production rate (qATP) in chemostat cultures of $L$. lactis (blue) and $L c$. mesenteroides (red). The dotted lines represent regression lines used to determine the maximum biomass yield on ATP $\left(Y_{X / A T P}{ }^{\max }\right)$ (1/slope) and the maintenance coefficient ( $\left.m_{\text {ATP }}\right)$ (intercept with $y$-axis). 


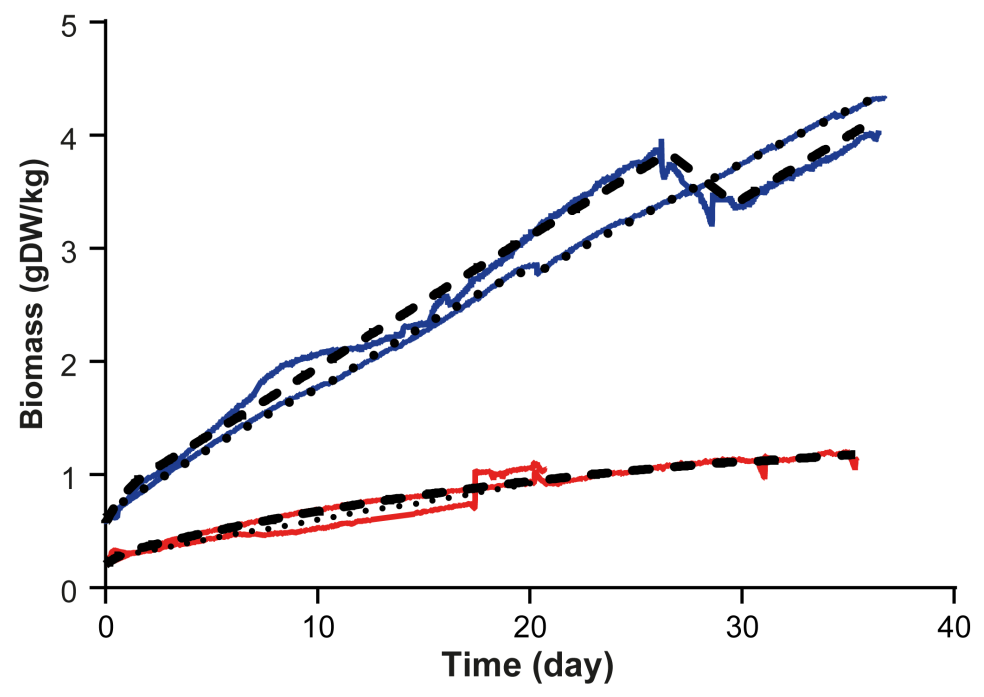

Figure 7.4: Model predictions of the biomass in retentostat mono-cultures of $L$. lactis (blue) and $L c$. mesenteroides (red). At time 0 a chemostat culture in steady state was switched to retentostat mode by insertion of a filter in the effluent line. The solid lines represent the online optical density measurements that were converted to cell dry weights with a secondorder polynomial function. The dashed and dotted lines represent the model predictions.

\section{Biomass accumulation on retentostat co-cultures}

The models of the retentostat mono-cultures were combined to predict growth of L. lactis and LC. mesenteroides in retentostat co-cultures. It was assumed that both species had similar substrate uptake kinetics, in other words that substrate was divided over both species depending on their biomass concentration. Moreover, it was assumed that cells did not lyse when the available substrate was less than their maintenance requirement. The model was compared with two independent retentostat co-cultures in which we determined i) the total cell dry weight and $\mathrm{ii}$ ) the relative abundance of both species. The relatively abundance was determined using selective plates: LM17 for L. lactis and MRS supplemented with vancomycin for $L c$. mesenteroides. Because we found an increase in viable but non culturable cells in retentostat mono-cultures of both L. lactis and LC. mesenteroides, we also determined the relative abundance using qPCR with a propidium monoazide (PMA) treatment (Erkus et al., 2016) to specifically amplify DNA of viable cells. Relative abundances of selective plating and qPCR were very similar (Suppl. Fig. S7.2). Compared to Lc. mesenteroides, the relative abundance of $L$. lactis increased during the retentostat co-cultivations from a ratio of 




Figure 7.5: Measured and predicted biomass accumulation of L. lactis (blue) and LC. mesenteroides (red) in retentostat co-cultures. At time 0 chemostat cultures of $L$. lactis and Lc. mesenteroides were combined and switched to retentostat mode by insertion of a filter in the effluent line. The total cell dry weight concentration (green symbols) was multiplied with the fraction of both species, determined by plates counts on selective plates (Suppl. Fig. S2), to obtain the estimated biomass concentration of $L$. lactis and $L C$. mesenteroides. Squares and circles represent measurements of two independent co-cultures. The lines represent the model predictions as explained in the materials and methods.

approximately $2.5: 1$ to $100: 1$. Based on the total cell dry weight and selective plate counts, dry weights of L. lactis cells and Lc. mesenteroides in the retentostat co-cultures were calculated and compared with our model prediction (Fig. 7.5). The model predicted the biomass concentration of both species very well indicating that competition was mainly at the level of nutrient acquisition and no growth stimulation or additional inhibiting interactions were found.

\section{Aroma formation in retentostat mono- and co-cultures}

During the retentostat mono- and co-cultures, samples were taken to analyse the production of volatile organic compounds (VOCs) by headspace SPME GC-MS. In total 47 aroma compounds were considered produced after exclusion of mediumassociated compounds (see materials and methods). To get an overview of the differences between aroma production by $L$. lactis and $L C$. mesenteroides, we performed a principal component analysis (PCA) using the aroma profiles of the mono-cultures (Fig. 7.6). 


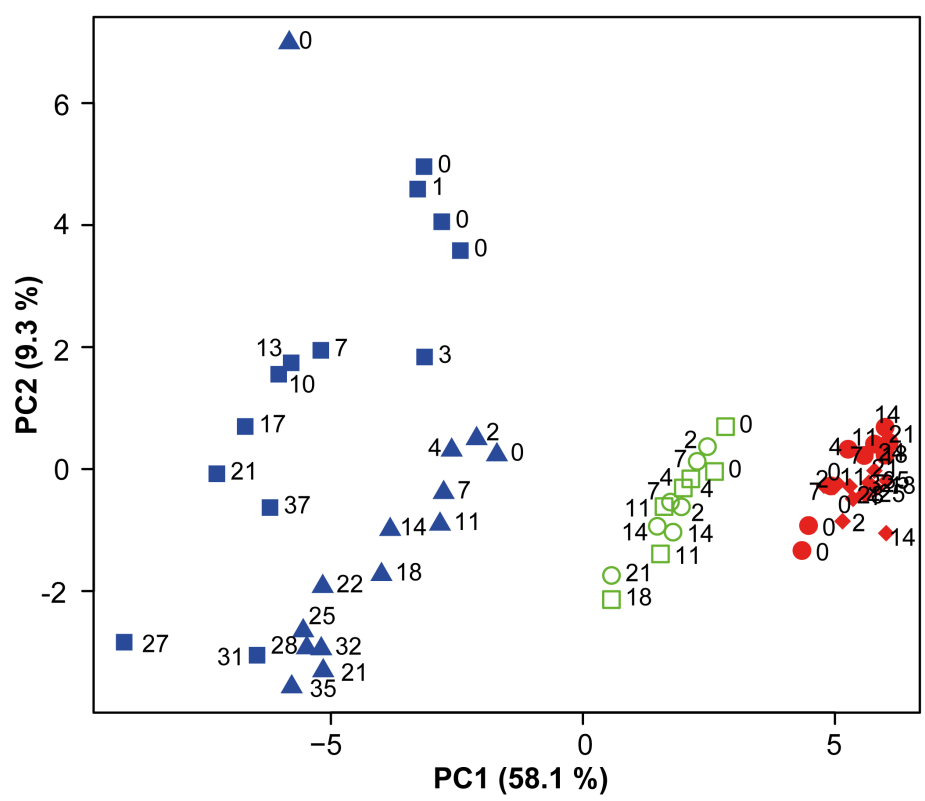

Figure 7.6: Principal component analysis (PCA) of aroma profiles produced in retentostat mono-cultures of $L$. lactis (blue) and LC. mesenteroides (red). The green symbols represent aroma profiles in retentostat co-cultures calculated based on the PCA analysis of the monocultures. Squares, triangles, diamonds and circles represent independent retentostat cultivations. The numbers near the symbols correspond to the time in the retentostat after inserting the filter in the effluent line. The $x$ and $y$-axis correspond to principal components 1 and 2, respectively, and the variance that they explained is given in parentheses.

Aroma profiles of $L$. lactis and $L c$. mesenteroides were clearly separated, indicating bigger differences in aroma production between the species than between the cultivations with the same species. A trend in time was visible for the $L$. lactis mono-cultures, indicating that aroma production changed at near-zero growth rates. Based on the PCA analysis of the mono-cultures, we calculated where the aroma profiles of the co-cultures were located in this PCA plot. Aroma profiles of the co-cultures were located in between the mono-cultures of $L$. lactis and $L C$. mesenteroides, indicating that Lc. mesenteroides contributed to formation of aroma compounds despite its low abundance. Furthermore, the aroma profiles of the co-cultures showed a similar trend in time as the mono-cultures of $L$. lactis, suggesting that the same aroma compounds increased and decreased as in the mono-cultures of $L$. lactis.

Comparison of the abundances of aroma compounds in mono-cultures revealed that 17 and 5 out of the 47 compounds were mainly produced at low growth rates by $L$. lactis and LC. mesenteroides, respectively (at least 8 -fold difference in maximum abundance between species) (Fig. 7.7). 


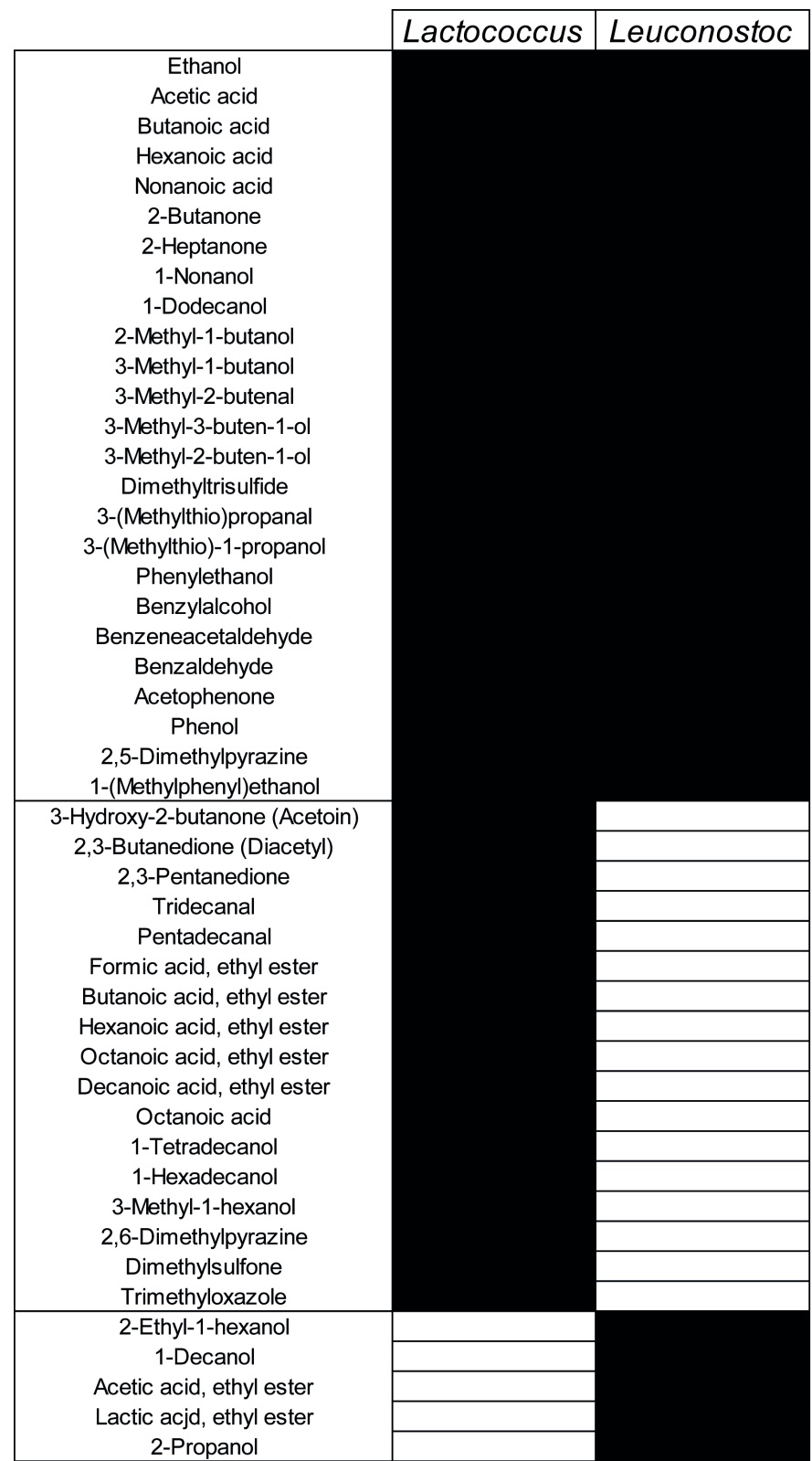

Figure 7.7: Presence (black) and absence (white) of the aroma compounds in monocultures of $L$. lactis and $L c$. mesenteroides.

All aroma compounds specific for $L$. lactis were found in the co-cultures, but the abundance of most fatty acid-related compounds was much lower in the cocultures compared to the mono-cultures of L. lactis (Fig. 7.8). This indicates that either Lc. mesenteroides affected the production of these compounds by L. lactis or these compounds were consumed by Lc. mesenteroides. 
Because only $1 \%$ of the population in the co-cultures was $L c$. mesenteroides, abundances of aroma compounds specific for $L c$. mesenteroides were expected to be lower than in the mono-cultures. Indeed, only 2 out of 5 compounds specific for Lc. mesenteroides, i.e. two medium-chain alcohols, were present in the cocultures at similar levels as in the mono-cultures (Fig. 7.8). No additional unique compounds were produced in the co-cultures indicating that interaction between the species at the level of metabolic complementation and/or cross-feeding did not result in additional aroma compounds.

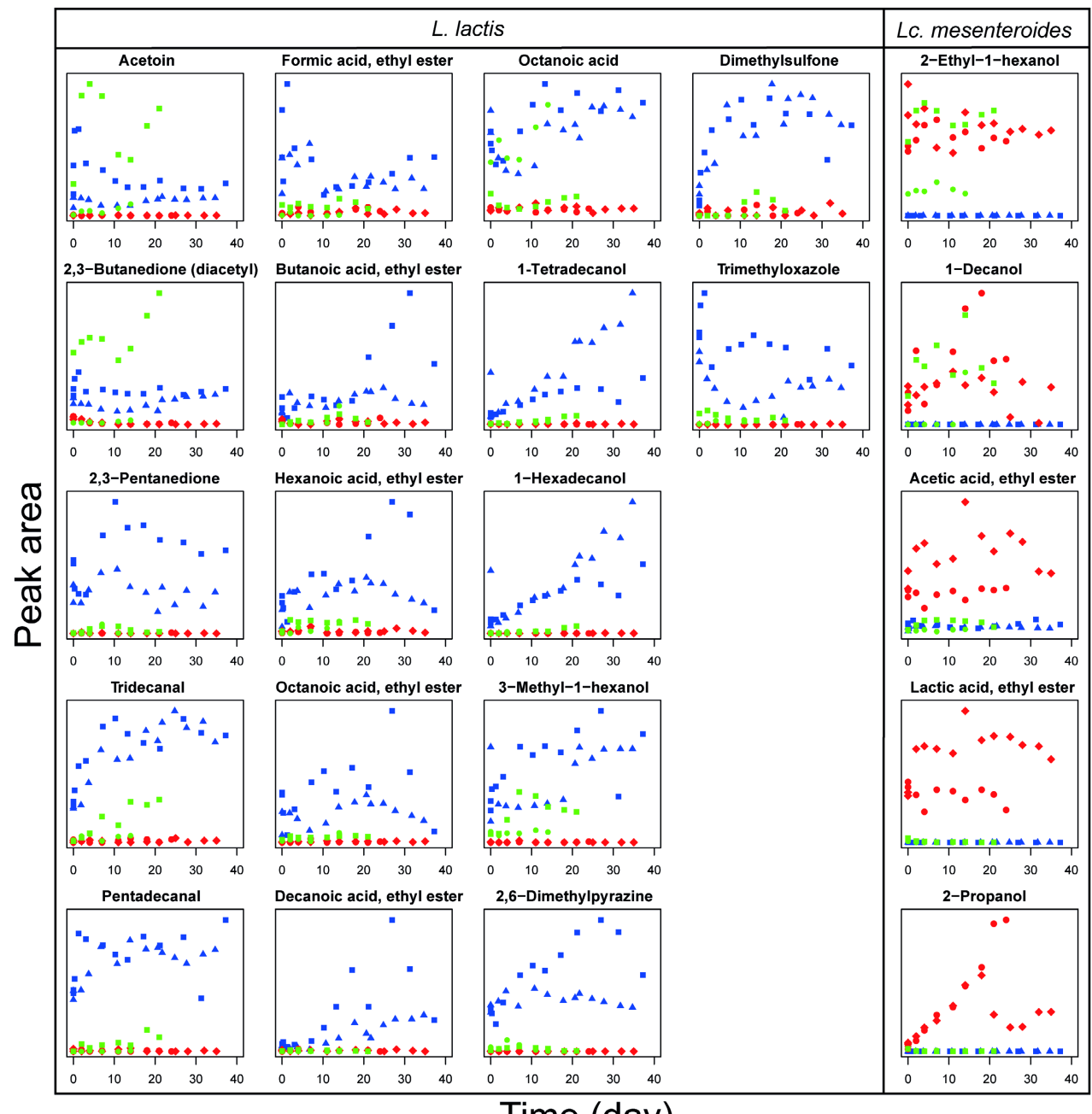

Time (day)

Figure 7.8: Quantitative comparison of the abundance of species-specific aroma compounds in retentostat mono-cultures of $L$. lactis (blue) and Lc. mesenteroides (red) and in co-cultures (green). Symbols represent independent cultivations. Only compounds that were specifically produced by one of the species in the mono-cultures are shown. 


\section{Discussion}

Lactococcus lactis biovar diacetylactis and Leuconostoc spp. are considered to be the main aroma producers in mesophilic DL-type starter cultures to make Dutchtype cheeses. During cheese ripening, these bacteria encounter long periods of extreme nutrient limitation severely reducing the growth rate. However, they can survive these periods while still contributing to flavour formation. In this study, aroma formation by $L$. lactis and LC. mesenteroides was studied at near-zero growth rates using retentostat cultivation. Moreover, both species were grown in retentostat co-cultures because this could potentially increase the aroma complexity by metabolic complementation (Erkus et al., 2013) similar to found for co-cultivation of yeasts (van Rijswijck et al., 2017).

In retentostat mono-cultures, the physiological response of both species towards the near-zero growth rates was similar. During 35 days of retentostat cultivation, the biomass concentration increased 8 - and 6-fold for L. lactis and LC. mesenteroides, respectively, while the growth rates decreased to less than $0.001 \mathrm{~h}^{-1}$. The viability remained above $80 \%$ throughout the cultivations. However, a large fraction of the L. lactis and Lc. mesenteroides cells lost the viability to grow on agar plates. Dynamic modelling of the biomass accumulation showed that the maintenance requirements of both $L$. lactis and $L C$. mesenteroides decreased 7-fold at near-zero growth rates compared to chemostat cultures. Most likely this was caused by a reduction in protein turnover, which is one of the major constituents of the maintenance requirements (Kempes et al., 2017, van Mastrigt et al., 2018b). In contrast, previous studies revealed that the maintenance requirement of $L$. lactis KF147, isolated from the nutrient-poor plant environment, did not decrease at near zero-growth rates (Ercan et al., 2013). Both strains used in the current study were directly isolated from the nutrient-rich dairy environment and therefore they might have evolved a similar response but a different response compared to the plant-derived L. lactis KF147.

The dynamic models of the mono-cultures were used to predict the biomass accumulation of both species in a retentostat co-culture and this was verified in two independent co-cultures. The model predicted the biomass accumulation in the co-cultures surprisingly well, despite the simple assumptions of distribution of substrates (lactose and citrate) over the species based on their biomass concentration and no growth stimulation or inhibiting interactions. This showed that both species competed mainly at the level of nutrient uptake and not by, for instance, bacteriocin-induced killing or mutualistic cross-feeding such as with yoghurt cultures (Settachaimongkon et al., 2014). It has been suggested that Leuconostoc promotes the growth of citrate-positive Lactococcus strains in 
fermented dairy products (Frantzen et al., 2017). However, no experimental support was given and such growth promotion could not be confirmed in this study.

$L$. lactis increased its abundance relative to $L c$. mesenteroides right from start and a ratio of approximately 100:1 was obtained at the end of the co-cultivations. This ratio corresponds well with ratios found in cheese (Erkus et al., 2013, Frantzen et al., 2017). The dominance of $L$. lactis can be explained by $i$ ) its more efficient metabolism (homofermentative versus heterofermentative), ii) its higher maximum biomass yield on ATP and iii) its lower maintenance requirements.

To determine if co-cultivation could enhance the aroma complexity and to identify compounds that were specific for $L$. lactis and Lc. mesenteroides, aroma compounds were quantified in mono- and co-cultures by HS SPME GC-MS. Compounds that were specific for $L$. lactis include mainly medium- and long-chain fatty acids and their derived products (alcohols, aldehydes, methyl ketones and esters), acetoin and diacetyl. Since no fat or fatty acids were present in the medium, it can be concluded that the medium- and long-chain fatty acids were synthesised by the bacteria. Compounds specific for $L c$. mesenteroides include small esters (ethyl lactate and ethyl acetate), 2-propanol, and two primary alcohols (1-decanol and 2-ethyl-1-hexanol). Ethanol, lactate and acetate were also abundant in mono-cultures of $L$. lactis, showing that the substrate specificities of the alcohol acyltransferases of Lc. mesenteroides FM06 and L. lactis FM03-V1 differ, the former being more active on small acids. The specific production of 2-propanol by LC. mesenteroides contrasts with the decreased amount in 2propanol observed in cheese to which Lc. mesenteroides was added (Pedersen et al., 2016).

Co-cultivation could result in increased aroma complexity as found for cocultivation of Saccharomyces cerevisiae and Cyberlindnera fabianii (van Rijswijck et al., 2017). In our study, specific Lc. mesenteroides aroma compounds were found in the co-cultures despite its low abundance (1\%), showing that retentostat co-cultivation could increase the aroma complexity compared to mono-cultures as both bacteria were retained. However, co-cultures lacked unique additional compounds. This might be caused by the large difference in abundance, which resulted in many $L$. lactis cells with few compounds specific for $L C$. mesenteroides and vice versa. The lack of unique compounds in co-cultures indicates that a similar aroma complexity can be obtained by combining two retentostat monocultures. This would allow for better control of the ratio of the species-specific aroma compounds and therefore might be preferred. Moreover, compounds that 
were only found in the mono-cultures of Lc. mesenteroides would be present in such a mixture as well contributing to the aroma complexity.

In conclusion, it is possible to grow $L$. lactis and $L c$. mesenteroides in retentostat co-cultures and both bacteria were retained and stayed viable throughout the cultivations. However, co-cultivation did not result in an increased aroma complexity, possibly due to the dominance of $L$. lactis. The dynamic models described in this study excellently predicted the biomass concentrations in the cocultures and could be a useful tool in selecting proper combinations of strains for retentostat co-cultivation.

\section{Acknowledgement}

This work was supported by Arla Foods (Aarhus, Denmark). 


\section{Supplementary materials}

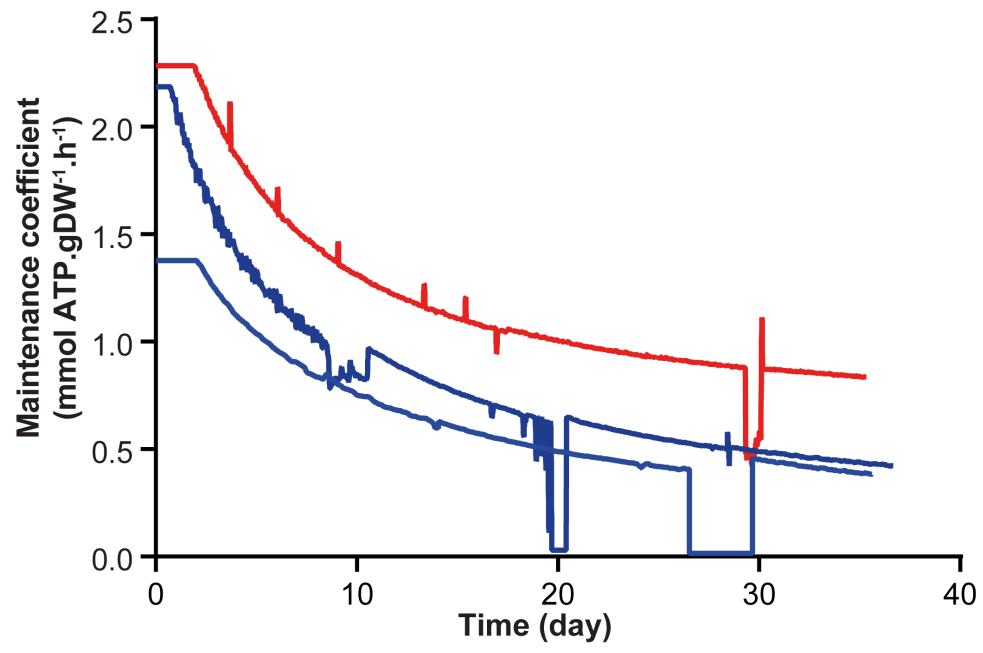

Supplementary Figure S7.1: Estimated maintenance coefficient $m_{\text {ATP }}$ of $L$. lactis (blue) and $L c$. mesenteroides (red) during retentostat mono-cultures. At time 0 a chemostat culture in steady state was switched to retentostat mode by insertion of a filter in the effluent line. The matp was estimated by mathematical modelling as explained in the materials and methods.
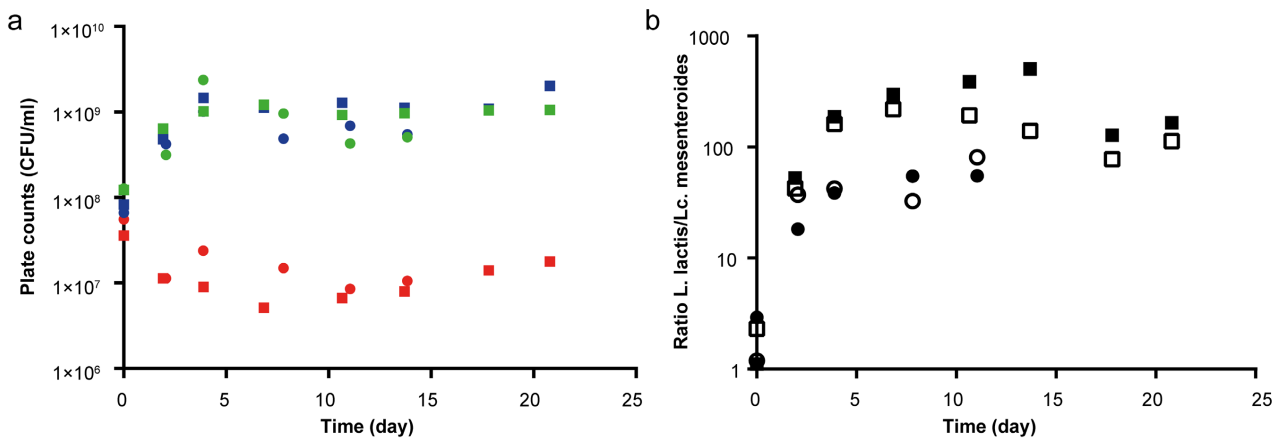

Supplementary Figure S7.2: Growth of L. lactis and LC. mesenteroides in retentostat cocultures. At time 0 chemostat cultures of $L$. lactis and $L C$. mesenteroides were combined and switched to retentostat mode by insertion of a filter in the effluent line. Squares and circles represent two independent retentostat co-cultures. A: Plates counts of L. lactis (blue) and Lc. mesenteroides (red) and both (green) on M17, MRS supplemented with vancomycin and MRS, respectively. B: Ratio of viable $L$. lactis and $L C$. mesenteroides determined by plate counts (open) and by qPCR combined with a propidium monoazide (PMA) treatment (filled). 


\section{References}

Boender, L. G. M., de Hulster, E. A. F., van Maris, A. J. A., Daran-Lapujade, P. A. S. and Pronk, J. T. (2009). Quantitative physiology of Saccharomyces cerevisiae at nearzero specific growth rates. Appl. Environ. Microbiol. 75(17): 5607-5614.

Cogan, T. M. and Jordan, K. N. (1994). Metabolism of Leuconostoc bacteria. J. Dairy Sci. 77(9): 2704-2717.

Ercan, O., Smid, E. J. and Kleerebezem, M. (2013). Quantitative physiology of Lactococcus lactis at extreme low-growth rates. Environ. Microbiol. 15(8): 2319-2332.

Erkus, O., de Jager, V. C. L., Spus, M., van Alen-Boerrigter, I. J., van Rijswijck, I. M. H., Hazelwood, L., Janssen, P. W. M., van Hijum, S. A. F. T., Kleerebezem, M. and Smid, E. J. (2013). Multifactorial diversity sustains microbial community stability. ISME J 7(11): 2126-2136.

Erkus, O., de Jager, V. C. L., Geene, R. T. C. M., van Alen-Boerrigter, I., Hazelwood, L., van Hijum, S. A. F. T., Kleerebezem, M. and Smid, E. J. (2016). Use of propidium monoazide for selective profiling of viable microbial cells during Gouda cheese ripening. Int. J. Food Microbiol. 228(Supplement C): 1-9.

Frantzen, C. A., Kot, W., Pedersen, T. B., Ardö, Y. M., Broadbent, J. R., Neve, H., Hansen, L. H., Dal Bello, F., Østlie, H. M., Kleppen, H. P., Vogensen, F. K. and Holo, H. (2017). Genomic characterization of dairy associated Leuconostoc species and diversity of Leuconostocs in undefined mixed mesophilic starter cultures. Front. Microbiol. 8: 132.

Hugenholtz, J. (1993). Citrate metabolism in lactic acid bacteria. FEMS Microbiol. Rev. 12(13): $165-178$

Kempes, C. P., van Bodegom, P. M., Wolpert, D., Libby, E., Amend, J. and Hoehler, T. (2017). Drivers of bacterial maintenance and minimal energy requirements. Front. Microbiol. 8(31).

Pedersen, T. B., Vogensen, F. K. and Ardö, Y. (2016). Effect of heterofermentative lactic acid bacteria of DL-starters in initial ripening of semi-hard cheese. Int. Dairy J. 57: 72-79.

Schmitt, P., Diviès, C. and Merlot, C. (1990). Utilization of citrate by Leuconostoc mesenteroides subsp. cremoris in continuous culture. Biotechnol. Lett. 12(2): 127130.

Settachaimongkon, S., Nout, M. J. R., Antunes Fernandes, E. C., Hettinga, K. A., Vervoort, J. M., van Hooijdonk, T. C. M., Zwietering, M. H., Smid, E. J. and van Valenberg, H. J. F. (2014). Influence of different proteolytic strains of Streptococcus thermophilus in co-culture with Lactobacillus delbrueckii subsp. bulgaricus on the metabolite profile of set-yoghurt. Int. J. Food Microbiol. 177: 29-36.

van Mastrigt, O., Abee, T. and Smid, E. J. (2017). Complete genome sequences of Lactococcus lactis subsp. lactis bv. diacetylactis FM03 and Leuconostoc mesenteroides FM06 isolated from cheese. Genome Announc. 5(28): e00633-17.

van Mastrigt, O., Gallegos Tejeda, D., Kristensen, M. N., Abee, T. and Smid, E. J. (2018a). Aroma formation during cheese ripening best resembled by slow-growing in retentostat cultures. Microb. Cell Fact. 17(1):104

van Mastrigt, O., Abee, T., Lillevang, S. K. and Smid, E. J. (2018b). Quantitative physiology and aroma formation of a dairy Lactococcus lactis at near-zero growth rates. Food Microbiol. 73: 216-226.

van Mastrigt, O., Mager, E. E., Jamin, C., Abee, T. and Smid, E. J. (2018c). Citrate, low pH and amino acid limitation induce citrate utilisation in Lactococcus lactis biovar diacetylactis. Microb. Biotechnol. 11(2): 369-380.

van Rijswijck, I. M. H., Wolkers - Rooijackers, J. C. M., Abee, T. and Smid, E. J. (2017). Performance of non-conventional yeasts in co-culture with brewers' yeast for steering ethanol and aroma production. Microb. Biotechnol. 10(6): 1591-1602.

Yvon, M. and Rijnen, L. (2001). Cheese flavour formation by amino acid catabolism. Int. Dairy J. 11(4): 185-201. 


\section{Chapter 8}

\section{Dynamics in copy numbers of five plasmids of a dairy Lactococcus lactis strain under dairy-related conditions including near-zero growth rates}

Oscar van Mastrigt, Marcel M. A. N. Lommers, Yorick C. de Vries, Tjakko Abee \& Eddy J. Smid

Published in Applied and Environmental Microbiology, 84:e00314-18 Published online: 23 March 2018, doi: 10.1128/AEM.00314-18 


\begin{abstract}
Lactic acid bacteria can carry multiple plasmids affecting their performance in dairy fermentations. The expression of plasmid-borne genes and the activity of the corresponding proteins are severely affected by changes in the numbers of plasmid copies. We studied the impact of growth rate on the dynamics of plasmid copy numbers at high growth rates in chemostat cultures and down to near-zero growth rates in retentostat cultures. Five plasmids of the dairy strain Lactococcus lactis FM03-V1 were selected, and these varied in size ( 3 to $39 \mathrm{~kb}$ ), in replication mechanism (theta or rolling-circle) and in putative (dairy-associated) functions. The copy numbers ranged from 1.5 to 40.5 and the copy number of theta-type replicating plasmids was negatively correlated to the plasmid size. Despite the extremely wide range of growth rates $\left(0.0003 \mathrm{~h}^{-1}\right.$ to $\left.0.6 \mathrm{~h}^{-1}\right)$, the copy numbers of the five plasmids were stable and only slightly increased at near-zero growth rates, showing that the plasmid replication rate was strictly controlled. One lowcopy-number plasmid, carrying a large exopolysaccharide gene cluster, was segregationally unstable during retentostat cultivations, reflected in a complete loss of the plasmid in one of the retentostat cultures. The copy number of the five plasmids was also hardly affected by varying the $\mathrm{pH}$ value, nutrient limitation, or the presence of citrate (maximum 2.2-fold), signifying the stability in copy number of the plasmids.
\end{abstract}




\section{Introduction}

The lactic acid bacterium Lactococcus lactis is extensively used as starter culture in dairy fermentations due to its high acidification rate and contribution to flavour and texture development in the fermented product. Important traits for the growth and survival in the milk environment are encoded by genes located on plasmids. This includes genes linked to lactose utilisation, casein hydrolysis, and bacteriophage and oxidative stress resistance (Górecki et al., 2011, Siezen et al., 2005), as well as genes involved in flavour and texture formation (e.g. citrate uptake and exopolysaccharide production) (Kranenburg et al., 1997, van Mastrigt et al., 2018a). Plasmids are extrachromosomal DNA molecules which can be lost and acquired and which can be exchanged within the population. Because plasmids replicate independently from the chromosome, their copy numbers (i.e. the number of plasmids per chromosome) can widely vary depending on, for instance, their replication mechanism, environmental conditions, and the genetic background of the strain carrying the plasmid. This could severely affect the total expression level of the genes they carry and the activity of the corresponding proteins.

Lactic acid bacteria encounter a wide variety of environmental conditions during industrial dairy processes, such as cheese production. $\mathrm{pH}$ values range from approximately 7 to 4 . Lactose is abundant in milk, but scarce during cheese ripening, and abundances of other nutrients, such as citrate, will change as well. This leads to high growth rates at the start of the fermentations, while growth rates severely reduce during cheese ripening. Because the plasmids carry essential functions for growth in milk, plasmids should be segregationally stable. Moreover, a constant plasmid copy number under a wide variety of conditions might be preferred for delivering a constant and predictable quality of the fermented product

The dairy isolate $L$. lactis FM03-V1 harbours 11 plasmids carrying genes encoding functions such as lactose and citrate utilisation, oligopeptide uptake, ion transport, bacteriophage resistance, heavy metal transport, stress resistance and polysaccharide production (Chapter 3 ). Of these 11 plasmids, there is one rollingcircle replicating plasmid ( $\mathrm{pLd} 3$ ) and 10 theta-type replicating plasmids.

In this study, the copy number of five plasmids of L. lactis FM03-V1 was monitored as a function of different environmental parameters relevant in cheese manufacturing including growth rate (0.6 to $\left.0.0003 \mathrm{~h}^{-1}\right), \mathrm{pH}$ (7 and 5.5), nutrient limitation (lactose or amino acid limitation) and the presence of citrate ( 0 or 10 $\mathrm{mM}$ ). 


\section{Materials and methods}

\section{Strain and growth medium}

For chemostat and retentostat cultivations, Lactococcus lactis subsp. lactis biovar diacetylactis FM03-V1 (van Mastrigt et al., 2018a) was pre-cultured as previously described (van Mastrigt et al., 2018b). The chemically defined media that were used for all cultivations contained $0.5 \%$ or $2 \%(\mathrm{w} / \mathrm{w})$ lactose, 0 or $10 \mathrm{mM}$ $\left(\mathrm{NH}_{4}\right)_{3}$ citrate, 0.1 or $1 \%$ Bacto-tryptone (van Mastrigt et al., 2018a) and were prepared in $20 \mathrm{~L}$ batches. The effect of growth rate was studied in a chemically defined medium containing $0.5 \%$ lactose, $10 \mathrm{mM}\left(\mathrm{NH}_{4}\right)_{3}$ citrate and $1 \%$ Bactotryptone.

\section{Chemostat and retentostat cultivations}

Chemostat and retentostat cultivations were performed in duplicate as previously described (van Mastrigt et al., 2018b). For the chemostat cultivations, the bacteria were grown at dilution rates between 0.05 and $0.6 \mathrm{~h}^{-1}$. The stirring speed was set at $300 \mathrm{rpm}$, the temperature was kept constant at $30^{\circ} \mathrm{C}$, and the $\mathrm{pH}$ was controlled at 5.5 or 7 by the automatic addition of $5 \mathrm{M} \mathrm{NaOH}$. The headspace was flushed with nitrogen gas at a rate of $0.1 \mathrm{~L} / \mathrm{min}$ to maintain anaerobic conditions. Samples were taken after reaching steady-state conditions, which were considered to be achieved after a minimum of five volume changes at which the optical density remained constant. Conditions for the retentostat cultivations were the same as for the chemostat cultivations, except that a stirring speed of $400 \mathrm{rpm}$ was used and the $\mathrm{pH}$ was set always at 5.5. After a steady state was achieved in a chemostat cultivation at a dilution rate of $0.025 \mathrm{~h}^{-1}$, retentostat cultivation was initiated by the insertion of a sterilisable polyethersulfone cross-flow filter (Spectrum laboratories, USA) in the effluent.

\section{Plasmid copy number determination}

The plasmid copy numbers were determined in two steps: i) DNA extraction followed by ii) quantitative PCR ( DNeasy Blood and Tissue kit (Qiagen, Germany) as previously described (van Mastrigt et al., 2018a). Subsequently, chromosomal and plasmid DNA were quantified with qPCR using specific primer sets (Table 8.1). 


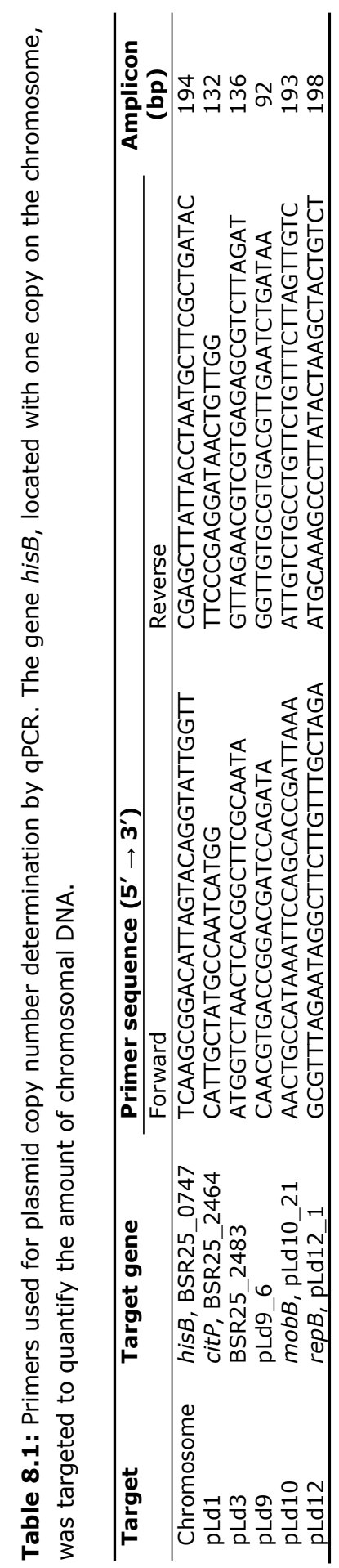


The PCRs took place in $20 \mu \mathrm{l}$ reaction mixtures containing $10 \mu \mathrm{l}$ iQ SYBR ${ }^{\circledR}$ Green Supermix (Bio-Rad, USA), $2 \mu \mathrm{l}$ purified DNA, $1 \mu \mathrm{M}$ forward primer, and $1 \mu \mathrm{M}$ reverse primer. The reactions started with an initial denaturation step at $95^{\circ} \mathrm{C}$ for 5 minutes followed by 39 cycles of $95^{\circ} \mathrm{C}$ for 30 seconds and $64^{\circ} \mathrm{C}$ for 30 seconds and finalised with a melting curve in which the temperature was raised from 65 to $95^{\circ} \mathrm{C}$ in steps of $0.5^{\circ} \mathrm{C}$ every 5 seconds to check for nonspecific amplification. The fluorescence of SYBR green was monitored, indicating the amount of doublestranded DNA (dsDNA). The threshold cycles in the qPCR were determined by regression using the Bio-Rad software CFX manager 3.1, which corrects for small differences in PCR efficiencies. DNA extractions were performed in duplicate and triplicate for chemostat and retentostat samples, respectively. Chromosomal and plasmid DNAs of each DNA extraction were quantified in triplicate and averaged for statistical analysis.

The plasmid copy number (PCN) was calculated from the threshold cycles for chromosomal $\left(\mathrm{Ct}_{\mathrm{c}}\right)$ and plasmid $\left(\mathrm{Ct}_{\mathrm{p}}\right)$ DNA using equation 8.1:

$$
P C N=2^{C t_{c}-C t_{p}}
$$

in which an efficiency of 2 was used because the threshold cycles were determined by regression, which already corrects for slightly lower or higher efficiencies.

\section{Statistical analysis}

To determine the effects of variables (growth rate and environmental conditions) on the plasmid copy numbers, statistical analyses were performed with $\mathrm{R}$ using the nlme package (Pinheiro et al., 2016). Linear mixed-effects models (Zuur et al., 2009) were fitted to the data to correct for the nested structure of the data: different conditions were analysed within the same cultivation and at least three DNA extractions were performed on the same sample. Therefore, DNA extraction nested within cultivation was used as a random categorical variable. For the fixed explanatory variables either growth rate or $\mathrm{pH}$, citrate, nutrient limitation, and their interactions were used. For retentostat cultivations, the growth rate was log transformed to make samples taken at high and low growth rates equally important. The determined plasmid copy numbers significantly correlated to the threshold cycle of the chromosome, which is a measure of how much chromosomal DNA was extracted. Therefore, the threshold cycle of the chromosome was added as a fixed explanatory variable to all models to correct for small changes in the amount of extracted DNA. First, a linear mixed-effects model (LME) with all fixed explanatory variables was compared with a generalised least squares model (GLS) by restricted maximum likelihood estimation (REML) to see if the LME was a better model, indicating that cells had more similar plasmid copy numbers within 
cultivations than between cultivations. Subsequently, the fixed explanatory variables of the LME containing all explanatory variables were optimised by a repetitive process of fitting a full model, dropping all allowable terms in turn, comparing the models with the maximum likelihood, dropping the least significant term, and repeating the whole process until all terms were significant ( $P$ value $<0.05$ ) (Zuur et al., 2009). The growth rate was never dropped as fixed factor. Finally, the final model was made by REML, and residual errors in final models were checked for homogeneity.

\section{Results}

The copy numbers were monitored for five plasmids of the dairy strain Lactococcus lactis FM03-V1. These five plasmids were chosen because they differ greatly in size (3.3 to $39.6 \mathrm{~kb}$ ), have two replication types ( 1 rolling-circle and 4 theta-type) and differ in putative encoded functions (Table 8.2). Plasmid pLd8 carrying a lactose operon was not included in the analysis because the cultures were lactoselimited with this disaccharide acting as the main carbon and energy source. This affects the stability of plasmid pLd8 as indicated by the loss of this plasmid only during growth in M17 supplemented with glucose (Chapter 3), which confirmed previous studies showing that the ability to ferment lactose was only maintained during propagation in the presence of lactose or galactose (McKay et al., 1972, Okulitch and Eagles, 1936). The dynamics in the plasmid copy numbers were analysed in two separate experiments varying either the growth rate or the environmental conditions ( $\mathrm{pH}$, nutrient limitation, presence of citrate). The effect of the growth rate on the plasmid copy numbers was studied using chemostat and retentostat cultures to cover an extremely wide range of growth rates $(0.0003$ to $0.6 \mathrm{~h}^{-1}$ ). To study the effect of environmental conditions, L. lactis was grown in chemostat cultivations at a constant growth rate $\left(0.135 \mathrm{~h}^{-1}\right)$. Because retentostat cultivation involves continuous filtration of the broth, a chemically defined medium was used in all experiments. This medium contained lactose, citrate and Bactotryptone (hydrolysed casein proteins) to represent the main energy, carbon and nitrogen sources in milk. All cultivations were performed in duplicate.

Table 8.2: Characteristics of the plasmids in L. lactis FM03-V1 used in this study. RCR: Rolling-circle replication.

\begin{tabular}{|c|c|c|c|c|}
\hline Plasmid & $\begin{array}{l}\text { Accession } \\
\text { numbers }\end{array}$ & $\begin{array}{l}\text { Size } \\
(\mathbf{k b})\end{array}$ & $\begin{array}{l}\text { Replication } \\
\text { type }\end{array}$ & Putative functions \\
\hline $\mathrm{pLd} 1$ & СР020605.1 & 8.3 & Theta & Citrate uptake \\
\hline pLd3 & СР020607.1 & 4.0 & $\mathrm{RCR}$ & Unknown \\
\hline pLd9 & MF150537.1 & 15.3 & Theta & Phage defence / D-lactate dehydrogenase \\
\hline pLd10 & MG813924.1 & 39.6 & Theta & Exopolysaccharide production \\
\hline pLd12 & MG813926.1 & 3.3 & Theta & Restriction-modification system \\
\hline
\end{tabular}




\section{Effect of growth rate}

L. lactis FM03-V1 was grown in chemostat cultures at high growth rates (0.05 to $\left.0.6 \mathrm{~h}^{-1}\right)$ and in retentostat cultures at extremely low growth rates (0.0003 to $\left.0.025 \mathrm{~h}^{-1}\right)$. Despite the near-zero growth rates, the bacteria remained viable ( $>80 \%$ ) as determined by live/dead staining with SYTO 9 and propidium iodide (van Mastrigt et al., 2018b). Linear mixed-effects modelling was used to analyse the effect of growth rate on the copy numbers of five plasmids. To correct for small differences in the amount of extracted DNA, which affects the plasmid extraction efficiency (data not shown), the threshold cycle of the chromosome was added as a fixed explanatory variable in the model. The plasmid copy numbers in the retentostat cultivations were analysed using a log transformation of the growth rate. During chemostat and retentostat cultivations, the plasmid copy numbers were not significantly affected or were only slightly affected with those of plasmids pLd1 (citrate uptake), pLd3 (rolling-circle) and pLd9 (bacteriophage resistance) increasing 1.7-, 3.5- and 1.6-fold at near-zero growth rates, respectively (Fig. 8.1, Table 8.3).

Notably, the largest plasmid, pLd10 (39.6 kb), was completely lost in retentostat culture 1 , and its copy number was significantly decreased in retentostat culture 2 compared to that in chemostat cultures ( 4.5 -fold). This shows that plasmid pLd10 was segregationally unstable at low growth rates, and the decreased copy number might be caused by the loss of the plasmid in a portion of the population. In general, the copy numbers of theta-type replicating plasmids were negatively correlated with the plasmid size $\left(R^{2}=0.921\right.$; Fig. 8.2) suggesting that bigger theta-type plasmids are less stable due to their lower copy number. Extrapolation of the linear relation between the size and copy number of theta-type replicating plasmids to the remaining six plasmids of $L$. lactis FM03-V1 gives a total plasmid content of $1.74 \mathrm{Mb}$, which is $72 \%$ of the amount of chromosomal DNA (2.43 Mb). 


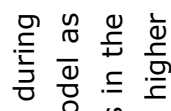

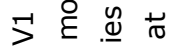

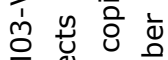

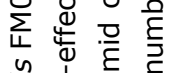

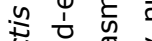

(0)

j $\bar{E}$ ¿

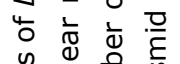

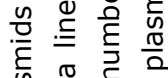

严

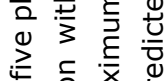

$\underset{4}{\gtrless}$ 드

० पू

㐫 它

E

ธลे

ते $\propto$ है

8등

凹

다



은 훙

ס ळ

단

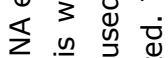

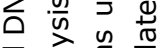

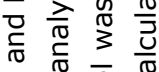

ए $\overline{0} \overline{0}$

त्र

टी

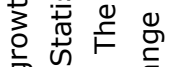

б ज ज

ข

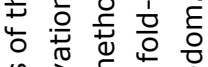

y $\geq \varepsilon \&$

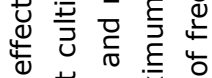

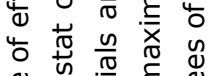

Uั

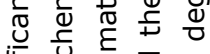

氜 몬

认ิ

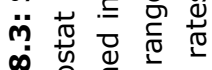

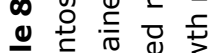



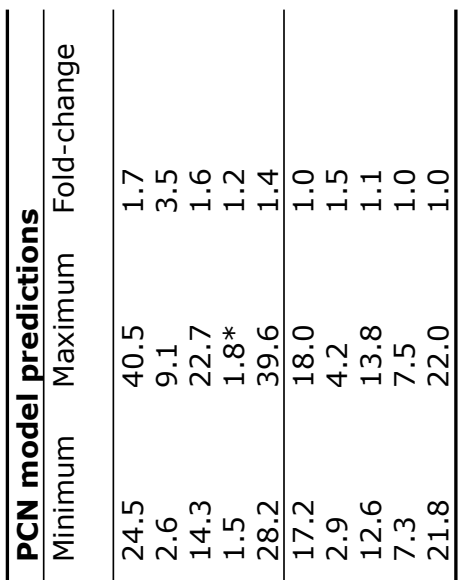

- 60

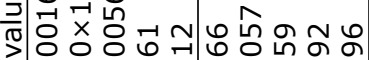

Q 0 N $0^{\circ} 0^{\circ} 0^{\circ} 0^{\circ} 0^{\circ}$

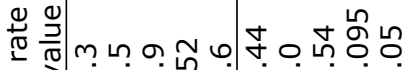

年

o

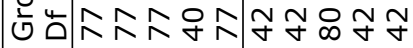

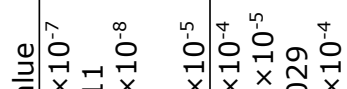

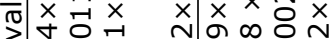

व

$\frac{2}{\frac{2}{2}}$

$\frac{3}{\pi} \ln 00 \quad m-10 N \infty$

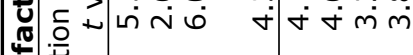

$\sqrt{\frac{0}{2}}$

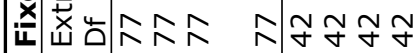

$\frac{\overline{0}}{\mathrm{E}}$

검 $m$ 움웍궉 $m$ 음웍

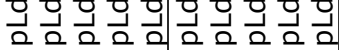

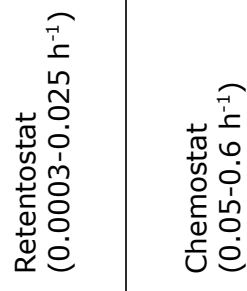




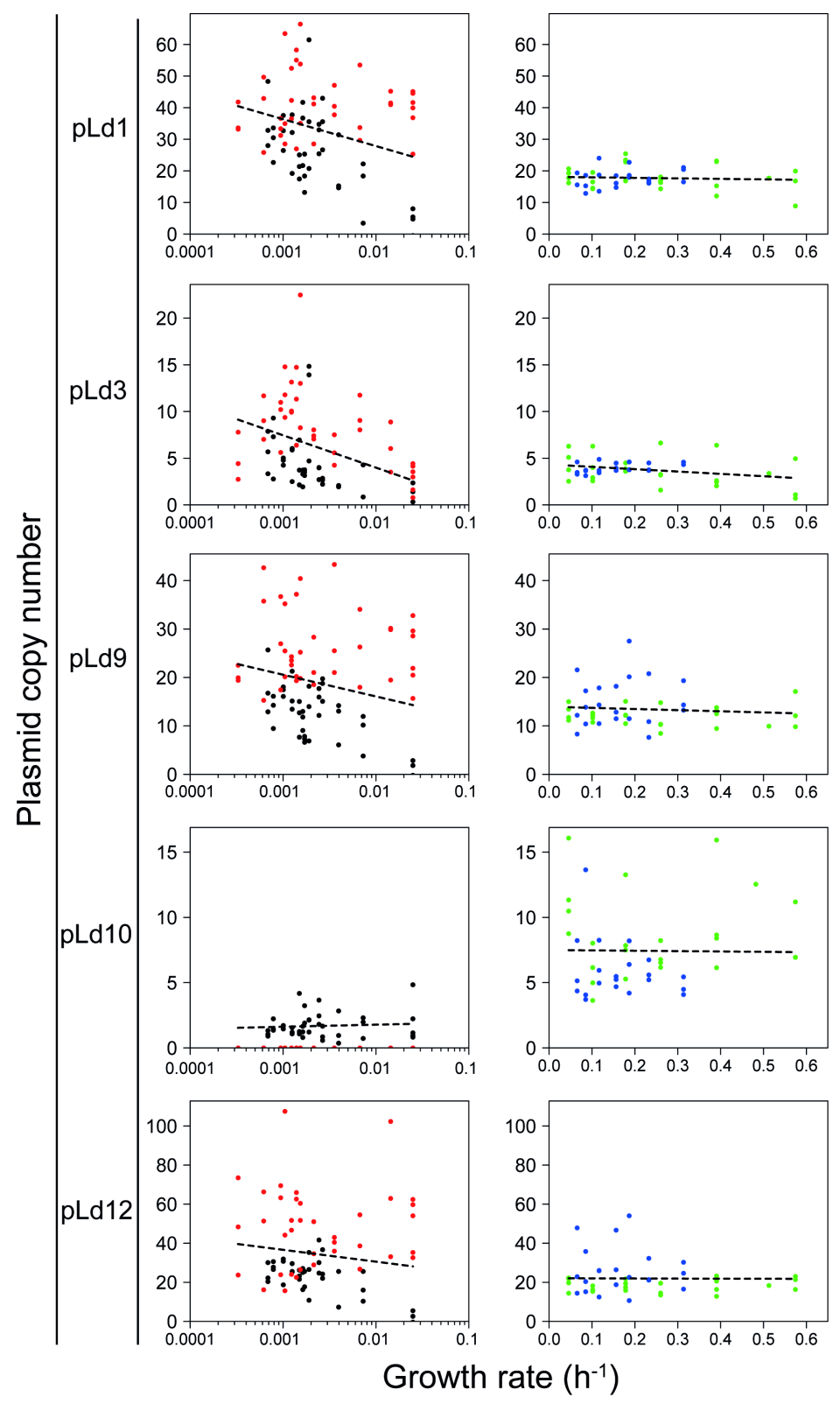

Figure 8.1: Copy numbers of five plasmids of L. lactis FM03-V1 in retentostat (left) and chemostat cultures (right). Red, black, blue and green represent independent retentostat cultures 1 and 2 and chemostat cultures 3 and 4, respectively. Every point represents the average plasmid copy number per DNA extraction calculated based on triplicate qPCR measurements. These plasmid copy numbers were corrected for slight changes in the amount of DNA extracted, which significantly affected the copy number measurement of most plasmids (Table 8.2). The dashed lines represent predictions calculated from linear mixed-effects model as explained in the materials and methods. 


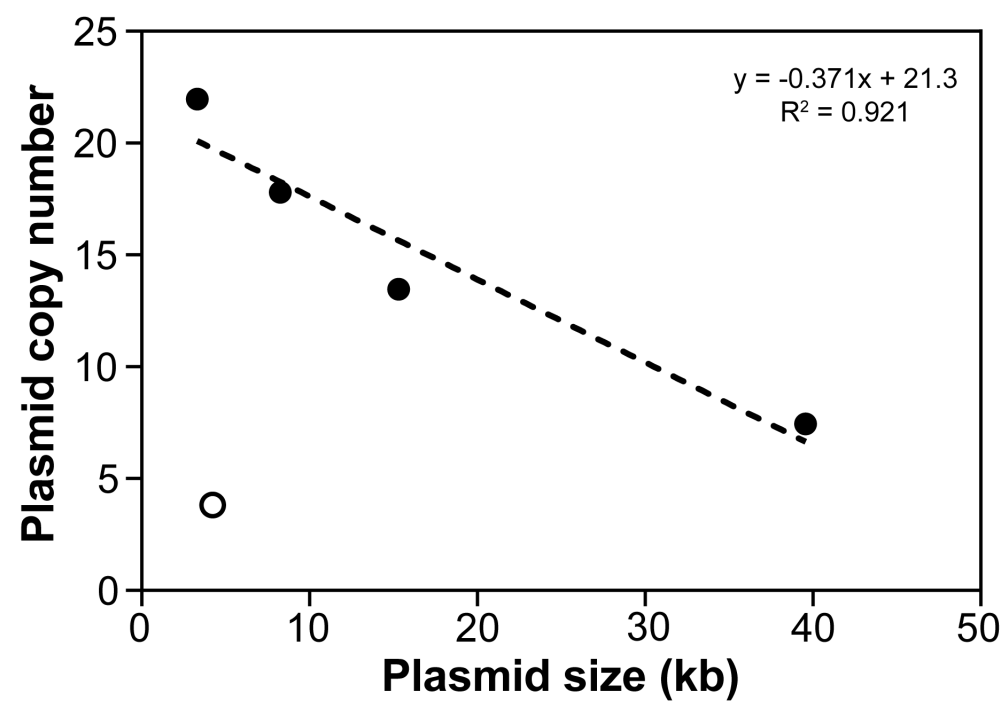

Figure 8.2: Correlation between the copy number and the size of the plasmids. Filled circles: theta-type replicating plasmids; open circles: rolling-circle replicating plasmid. Plasmid copy numbers were calculated at a growth rate of $0.2 \mathrm{~h}^{-1}$ based on the obtained model for chemostat cultivations.

\section{Effect of environmental conditions}

To study the effects of $\mathrm{pH}$, nutrient limitation and the presence of citrate in the medium on plasmid copy numbers, L. lactis FM03-V1 was grown in chemostat cultures at a constant growth rate $\left(0.135 \mathrm{~h}^{-1}\right)$ at $\mathrm{pH} 5.5$ and 7 , with and without supplementation of citrate and under lactose and amino acid limitation. These environmental parameters were chosen to represent dairy environments during fermentation: milk contains approximately $10 \mathrm{mM}$ citrate (Garnsworthy et al., 2006); milk is acidified from approximately pH 7 to 5 (Fox and McSweeney, 2017) by lactic acid bacteria and amino acids or lactose might be limiting depending on the lactose uptake kinetics and the proteolytic capacity of the starter culture. Linear mixed-effects models were used to determine the effect of each parameter on the copy number of each plasmid. The copy numbers of plasmids pLd1 (citrate uptake) and pLd10 (EPS production) were significantly lower at low pH (2.0- and 1.9-fold, respectively), while the copy number of plasmid pLd3 (rolling-circle) was lower under amino acid limitation (2.2-fold) (Fig. 8.3, Table 8.4). The copy numbers of the remaining two plasmids were not significantly affected under any of the tested conditions. 


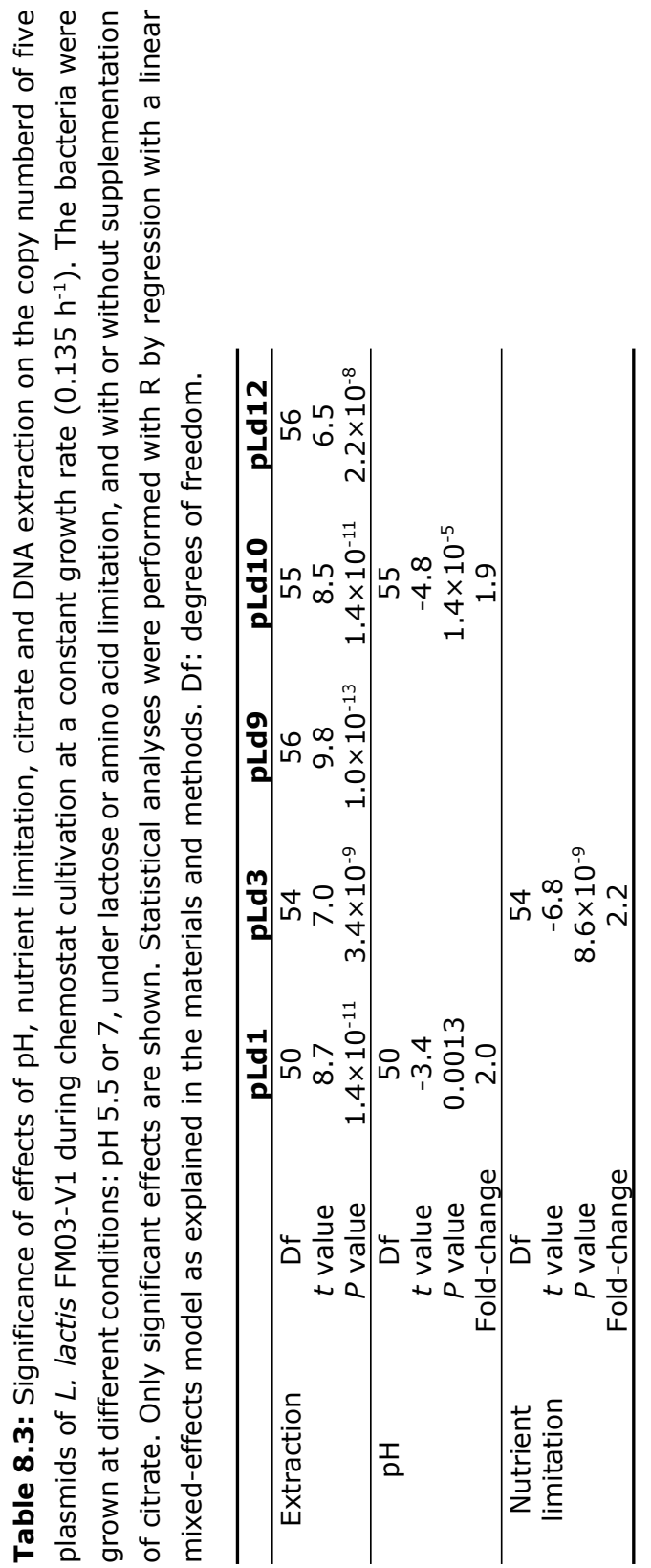






Figure 8.3: Copy numbers of five plasmids of L. lactis FM03-V1 during chemostat cultivation at a constant growth rate $\left(0.135 \mathrm{~h}^{-1}\right)$ at different conditions: $\mathrm{pH} 5.5$ or 7 , under lactose or amino acid limitation, and with (+) or without (-) supplementation of citrate. Boxplots were made with the average plasmid copy numbers per DNA extraction, which were calculated based on triplicate qPCR measurements. These plasmid copy numbers were corrected for slight changes in the amount of DNA extracted, which significantly affected the copy number measurements of most plasmids (Table 8.3). Triangles represent the predictions of linear mixed-effects models as explained in the materials and methods. $P$ values of significant effects are indicated in the plots. 


\section{Discussion}

To assess the dynamics in the copy numbers of five plasmids, Lactococcus lactis FM03-V1 was grown in environments that reflect the conditions encountered during dairy fermentations. The copy numbers of plasmids were studied at nearzero growth rates using retentostat cultivation. Differences in plasmid copy numbers were limited ( $<4$-fold) compared to the extremely wide range of growth rates ( $>1000$-fold). This shows that the replication rate of the plasmids is strictly coupled to the growth rate. It has been demonstrated that the plasmid replication rate is controlled by negative regulatory systems responding to fluctuations in the copy number as extensively reviewed by del Solar et al. (1998) and Chattoraj (2000). More specifically, these systems respond to fluctuations in plasmid concentrations. Because the cell volume slightly increases at near-zero growth rates (van Mastrigt et al., 2018b), a constant plasmid concentration implies a slightly higher number of plasmids per cell in bigger cells. Since these bigger cells still contain only one chromosomal copy, increased cell volumes could explain the increased copy numbers of plasmids pLd1, pLd3 and pLd9 at near-zero growth rates.

Despite the strict regulation of the plasmid copy numbers, plasmids can still be lost under specific conditions as illustrated by the complete loss of plasmid pLd10 in retentostat culture 1 and the significantly decreased apparent copy number in retentostat culture 2 , which might be caused by the loss of the plasmid in a portion of the population. This plasmid carries a large eps gene cluster. Exopolysaccharide (EPS) production is an expensive process in terms of energy usage and we speculate that the extreme energy limitation in retentostat cultivation might have selected for cells that did not produce EPS due to loss of plasmid pLd10. The energy advantage of cells that have lost plasmid pLd10 enabled these cells to grow at lower substrate availabilities resulting in the dominance of these cells in the retentostat cultures.

The mode of replication has been suggested to be a major factor in the segregational stability of plasmids in L. lactis, with theta-type replication plasmids being more stable (Kiewiet et al., 1993). In this study, a theta-type replicating plasmid (pLd10) was lost in one of the retentostat cultivations, while the rollingcircle replicating plasmid was maintained. Plasmid pLd10 has a relatively low copy number compared to those of the other plasmids, which increases its chance to be lost during cell division. We found that the copy number of theta-type replicating plasmids was negatively correlated to the plasmid size. A similar relation was also found for plasmids of Bacillus thuringiensis YBT-1520 (Zhong et 
al., 2011) and suggests a lower stability for larger plasmids due to their lower copy number.

The amount of chromosomal DNA in the extraction could affect the plasmid extraction efficiency and thereby plasmid copy number measurement. Therefore, the threshold cycle of the chromosomal DNA was included as fixed explanatory variable in the linear mixed-effects models to correct for small changes in the amount of extracted DNA. This variable appeared to be significant in most analyses (Tables 8.2 and 8.3 ) and improved the reliability of the results. This demonstrates that to obtain reliable results with the described copy number determination method, standardisation of the amount of cells in the DNA extraction and/or correction for small changes in the amount of extracted DNA, as implemented in the current study, is required.

In addition to the effect of growth rate on the plasmid copy number, we analysed the effects of several environmental parameters, which commonly change during dairy fermentations: $\mathrm{pH}$, nutrient limitation and the presence of citrate. In general, their effects on the plasmid copy numbers were very small (maximum 2.2-fold), signifying the stability of the plasmid copy numbers. Comparisons of the plasmid copy numbers in the chemostat cultures at different dilution rates (Fig. 8.1) and under different environmental conditions (Fig. 8.3) showed that the determined copy numbers in the latter chemostat cultures were approximately 2fold lower. This can be explained by the slightly larger amount of chromosomal DNA that was extracted on average in these samples (chromosomal threshold cycle of 12.5 to 15 compared to 15 to 17 ) because we only corrected for the amount of chromosomal DNA within experiments, i.e. conditions analysed in the same linear mixed-effects model, and not between experiments. This demonstrates the importance of standardisation of the amount of cells in the DNA extraction for comparisons between experiments.

The copy number of plasmid pLd1 carrying the citQRP operon required for citrate uptake decreased 2.0 -fold at low $\mathrm{pH}$, while the nutrient limitation and the presence of citrate did not have a significant effect. In contrast, earlier studies (van Mastrigt et al., 2018a) revealed a slight increase (1.4-fold) in the copy number of plasmid pLd1 at pH 5.5 compared to that at pH 7. This discrepancy shows that such small differences in plasmid copy number ( $<5$ plasmid copies) cannot be reproducibly found with the applied technique and should be considered not significant. This is signified by the relatively small changes compared to the increase in citrate utilisation rate at low pH in the presence of citrate, which can mount up to 65-fold (van Mastrigt et al., 2018a). 
In conclusion, plasmid copy numbers were remarkably stable under different environmental conditions relevant for cheese manufacturing, which leads to a constant and predictable product quality in industrial processes.

\section{Acknowledgement}

This work was supported by Arla Foods (Aarhus, Denmark). 


\section{References}

Chattoraj, D. K. (2000). Control of plasmid DNA replication by iterons: no longer paradoxical. Mol. Microbiol. 37(3): 467-476.

del Solar, G., Giraldo, R., Ruiz-Echevarría, M. J., Espinosa, M. and Díaz-Orejas, R. (1998). Replication and control of circular bacterial plasmids. Microbiol. Mol. Biol. Rev. 62(2): 434-464.

Fox, P. F. and McSweeney, P. L. H. (2017). Cheese: an overview. In McSweeney, P. L. H., Fox, P. F., Cotter, P. and Everett, D. W. (Eds.), Cheese: chemistry, physics and microbiology (4th ed., Vol. 1, pp. 5-21). San Diego: Academic Press.

Garnsworthy, P. C., Masson, L. L., Lock, A. L. and Mottram, T. T. (2006). Variation of milk citrate with stage of lactation and de novo fatty acid synthesis in dairy cows. $J$. Dairy Sci. 89(5): 1604-1612.

Górecki, R. K., Koryszewska-Bagińska, A., Gołębiewski, M., Żylińska, J., Grynberg, M. and Bardowski, J. K. (2011). Adaptative potential of the Lactococcus lactis IL594 strain encoded in its 7 plasmids. PLoS One 6(7): e22238.

Kiewiet, R., Kok, J., Seegers, J. F. M. L., Venema, G. and Bron, S. (1993). The mode of replication is a major factor in segregational plasmid instability in Lactococcus lactis. Appl. Environ. Microbiol. 59(2): 358-364.

Kranenburg, R. v., Marugg, J. D., Van Swam, I. I., Willem, N. J. and De Vos, W. M. (1997). Molecular characterization of the plasmid-encoded eps gene cluster essential for exopolysaccharide biosynthesis in Lactococcus lactis. Mol. Microbiol. 24(2): 387397.

McKay, L., Baldwin, K. and Zottola, E. (1972). Loss of lactose metabolism in lactic streptococci. Appl. Microbiol. 23(6): 1090-1096.

Okulitch, O. and Eagles, B. A. (1936). Cheese-ripening studies: The influence of the configurational relations of the hexoses on the sugar-fermenting abilities of lactose acid streptococci: A preliminary note. Canadian Journal of Research 14b(9): 320324.

Pinheiro, J., Bates, D., DebRoy, S., Sarkar, D. and Team, R. C. (2016). nlme: Linear and nonlinear mixed-effects models. Retrieved 5th February 2018, from http://CRAN.R-project.org/package =nlme

Siezen, R. J., Renckens, B., van Swam, I., Peters, S., van Kranenburg, R., Kleerebezem, M. and de Vos, W. M. (2005). Complete sequences of four plasmids of Lactococcus lactis subsp. cremoris SK11 reveal extensive adaptation to the dairy environment. Appl. Environ. Microbiol. 71(12): 8371-8382.

van Mastrigt, O., Mager, E. E., Jamin, C., Abee, T. and Smid, E. J. (2018a). Citrate, low pH and amino acid limitation induce citrate utilisation in Lactococcus lactis biovar diacetylactis. Microb. Biotechnol. 11(2): 369-380.

van Mastrigt, O., Abee, T., Lillevang, S. K. and Smid, E. J. (2018b). Quantitative physiology and aroma formation of a dairy Lactococcus lactis at near-zero growth rates. Food Microbiol. 73: 216-226.

Zhong, C., Peng, D., Ye, W., Chai, L., Qi, J., Yu, Z., Ruan, L. and Sun, M. (2011). Determination of plasmid copy number reveals the total plasmid DNA amount is greater than the chromosomal DNA amount in Bacillus thuringiensis YBT-1520. PLoS One 6(1): e16025.

Zuur, A. F., Ieno, E. N., Walker, N. J., Saveliev, A. A. and Smith, G. M. (2009). Mixed effects models and extensions in ecology with $R$. New York, USA: Springer. 



\section{Chapter 9}

\section{Application of a partial cell recycling chemostat for continuous production of aroma compounds at near-zero growth rates}

Oscar van Mastrigt, Reinier A. Egas, Søren K. Lillevang, Tjakko Abee \& Eddy J. Smid 


\begin{abstract}
The partial cell recycling chemostat is a modification of the chemostat in which cells are partially fed back into the bioreactor making it possible to use dilution rates higher than the growth rate. This results in higher biomass concentrations and increased process rates, which is especially useful for dilute or insoluble substrates and to prevent product inhibition. For the first time, we show that this system could also be used to produce compounds that are formed at near-zero growth rates, such as many aroma compounds typical for ripened cheese. In this study, Lactococcus lactis FM03-V2 was cultivated in a partial cell recycling chemostat at growth rates between 0.0025 and $0.025 \mathrm{~h}^{-1}$. Detailed analysis of aroma compounds produced by L. lactis FM03-V2 revealed that levels of particular compounds were clearly affected by the growth rate within the studied range demonstrating that we can steer the aroma production by changing the growth rate. With this approach, we also experimentally validated that the maintenance coefficient of this dairy strain decreased at near-zero growth rates (6.4-fold). An exponential decreasing maintenance coefficient was included in the growth model and this enabled accurate prediction of biomass accumulation in the partial cell recycling chemostat. This study demonstrates the potential of partial cell recycling chemostat both as aroma production system at near-zero growth rates and as unique research tool.
\end{abstract}




\section{Introduction}

The partial cell recycling chemostat, also called chemostat with feedback, was first presented by Herbert (Herbert, 1961) and first experiments with this technique have been published by Pirt and Kurowski (Pirt and Kurowski, 1970). This system is a modification of the chemostat, in which biomass is partially recycled by means of centrifugation, filtration or gravity sedimentation (Pirt and Kurowski, 1970). Thereby, dilution rates higher than the growth rate can be used resulting in higher biomass concentrations and increased process rates. In particular, this can be useful for fermentations with dilute or insoluble substrates or in the presence of inhibitory substances (Bibal et al., 1991, Bull and Young, 1981, Pirt, 1975).

With the advances in membrane technology, it is now possible to have two effluents, one with complete biomass retention and one without biomass retention enhancing the applicability of this system. In this situation, the ratio of the flow rate of the cell-free permeate over the total effluent flow, called the recycle ratio $R$, together with the dilution rate $D$ determines the growth rate $\mu$ :

$$
\mu=(1-R) \cdot D \leq D
$$

When $\mathrm{R}=0$, there is no cell recycling and the system is a conventional chemostat in which the growth rate $\mu$ equals the dilution rate. When $\mathrm{R}=1$, there is complete cell recycling, the growth rate approaches 0 and there is no steady state possible.

Because both the dilution rate and recycle ratio can be controlled, the growth rate can be controlled even at extremely low growth rates that would cause homogeneity problems in conventional chemostat cultures. This feature shows two new applications of the partial cell recycling chemostat that are yet unexplored: i) studying microbes at near-zero growth rates in true steady states and ii) production of metabolites typical for near-zero growth rates, such as aroma compounds formed during cheese ripening.

In the last decade, the effect of near-zero growth rates on the physiology of several microbes has been studied using retentostat cultivation, which uses complete cell recycling and therefore never leads to a true steady state (Boender et al., 2009, Ercan et al., 2013, Goffin et al., 2010, Overkamp et al., 2015, Panikov et al., 2015, van Mastrigt et al., 2018a). Moreover, the growth rate quickly decreases to $<0.005 \mathrm{~h}^{-1}$ in retentostat cultures, making it impossible to properly study growth rates between approximately 0.005 and $0.025 \mathrm{~h}^{-1}$ using retentostat cultures. Recently, dynamic modelling of retentostat cultures revealed a 7-fold reduction in the maintenance requirement of Lactococcus lactis FM03-V1 after 35 days of cultivation (van Mastrigt et al., 2018a). Moreover, formation of particular 
aroma compounds increased during the cultivations resembling aroma formation during cheese ripening.

In this study, the dairy L. lactis FM03-V2 was grown in partial cell cycling chemostat cultures at low growth rates $\left(0.0025-0.025 \mathrm{~h}^{-1}\right)$ to determine if the changes in maintenance requirement and aroma formation were caused by the low growth rates. Moreover, additional applications of the partial cell recycling chemostat are discussed.

\section{Materials and methods}

\section{Strain and medium}

In this study Lactococcus lactis subsp. lactis FM03-V2 was used, which is a single colony isolate of $L$. lactis FM03-V1 lacking plasmid pLd1 carrying the citQRP operon. For the continuous cultivation, bacteria were pre-cultured as previously described (van Mastrigt et al., 2018a). Briefly, a single colony from an M17 agar plate supplemented with $0.5 \%(\mathrm{w} / \mathrm{v})$ lactose was cultured overnight at $30^{\circ} \mathrm{C}$ on lactose-limited chemically defined medium (van Mastrigt et al., 2018b). Subsequently, the culture was used to inoculate the bioreactor at $1 \%(\mathrm{v} / \mathrm{v})$ inoculation level. For the continuous cultivation a chemically defined medium was used containing $0.5 \%(\mathrm{w} / \mathrm{w})$ lactose, $10 \mathrm{mM}\left(\mathrm{NH}_{4}\right)_{3}$ citrate and $1 \%$ Bacto-tryptone as main carbon and nitrogen sources (van Mastrigt et al., 2018b).

\section{Partial cell recycling chemostat cultivation}

Bacteria were grown in partial cell recycling chemostat cultures at growth rates between 0.0025 and $0.025 \mathrm{~h}^{-1}$ in bioreactors with a working volume of $1 \mathrm{~L}$ (Multifors, Infors HT, Switzerland). A constant dilution rate of $0.025 \mathrm{~h}^{-1}$ was used and the growth rate was varied by changing the recycle ratio by removing part of the effluent via a polyethersulfone cross-flow filter $(0.65 \mu \mathrm{m}$, Spectrum laboratories, USA). The temperature was kept constant at $30^{\circ} \mathrm{C}$, the $\mathrm{pH}$ was controlled at 5.5 by automatic addition of $5 \mathrm{M} \mathrm{NaOH}$ and anaerobic conditions were maintained by flushing the headspace with nitrogen gas at a rate of 0.1 $\mathrm{L} / \mathrm{min}$. The stirring speed was set at $400 \mathrm{rpm}$. The optical density at $600 \mathrm{~nm}$ was continuously monitored using an internal probe (Trucell2, Finesse, USA). Samples were taken after reaching steady state conditions, which were considered to be achieved when the online optical density did not change significantly for approximately 2 days. Rates of the feed, effluent and cell-free permeate were determined by continuously weighing the medium and waste vessels. 


\section{Biomass determination}

The cell dry weight was determined in triplicate as previously described (van Mastrigt et al., 2018b). Briefly, $3 \mathrm{~mL}$ samples was passed through a preweighted $0.2 \mu \mathrm{m}$ membrane filter (Pall Corporation, USA) by a vacuum filtration unit, dried at $80^{\circ} \mathrm{C}$ for 2 days and weighted again.

\section{Cell viability}

The viability of cells in the culture was determined by LIVE/DEAD Baclight Bacterial Viability kit (Molecular Probes Europe, The Netherlands). $100 \mu$ l culture was incubated with $3.34 \mu \mathrm{M}$ green fluorescent SYTO 9 and $20 \mu \mathrm{M}$ red fluorescent propidium iodide for 10 minutes at room temperature in the dark. A fluorescent microscope was used for visualisation and estimation of the number of green and red cells.

\section{Extracellular metabolites analysis}

Lactose, citrate, lactate, acetate, ethanol, formate, pyruvate and acetoin were quantified by High Performance Liquid Chromatography (HPLC) as previously described (van Mastrigt et al., 2018b).

\section{Volatile organic compounds analysis}

Volatile organic compounds were analysed by headspace solid phase microextraction gas chromatography mass spectrometry (HS SPME GC-MS) as previously described (van Mastrigt et al., 2018a). Aroma profiles were analysed with Chromeleon 7.2 software. The ICIS algorithm was used for peak integration and mass spectra of these peaks were compared with the NIST main library for identification. One quantifying peak (either the highest $\mathrm{m} / \mathrm{z}$ peak per compound or a high $\mathrm{m} / \mathrm{z}$ peak that was not present in overlapping compounds) was selected for quantification of the compound and 1 or 2 confirming peaks were used for confirmation.

\section{Modelling}

To estimate the maintenance coefficient during the partial cell recycling chemostat cultivation, biomass accumulation was modelled based on: i) mass balances of biomass and substrate (Eq. 9.2 and 9.3), ii) the Herbert-Pirt equation (Eq. 9.4) describing how substrate is divided over growth and maintenance, and iii) changes in metabolism resulting in different ATP yield on substrate (Eq. 9.5 and 9.6). In 
these equations, $C_{x}$ denotes biomass concentration in the reactor ( $\left.g D W / k g\right), \mu$ is the growth rate $\left(h^{-1}\right), F$ is the flow of medium $(L / h), V$ is the volume $(L), R$ is the recycle ratio, $\mathrm{C}_{\mathrm{s} \text {,out }}$ is the substrate concentration in the reactor (no residual lactose), $\mathrm{C}_{\mathrm{s}, \mathrm{in}}$ is the substrate concentration in the medium $(175.2 \mathrm{mCmol} / \mathrm{L}$ lactose), $\mathrm{q}_{\mathrm{s}}$ is the biomass specific substrate consumption rate $\left(\mathrm{Cmol}_{\mathrm{gDW}} \mathrm{gDW}^{-1} \cdot \mathrm{h}^{-1}\right)$, $\mathrm{t}$ is the time $(\mathrm{h}), Y_{x / s}^{\max }$ is the maximum biomass yield on substrate (gDW/Cmols), $Y_{x / A T P}^{\max }$ is the maximum biomass yield on ATP ( $\mathrm{gDW} / \mathrm{mol}$ ATP), $\mathrm{m}_{\mathrm{s}}$ is the substraterelated maintenance coefficient (CmolS.gDW $\left.{ }^{-1} \cdot \mathrm{h}^{-1}\right), \mathrm{m}_{\text {ATP }}$ is the energy-related maintenance coefficient (mol ATP.gDW ${ }^{-1} \cdot \mathrm{h}^{-1}$ ) and $\mathrm{Y}_{\mathrm{ATP} / \mathrm{s}}$ is the ATP yield on substrate (mol ATP/CmolS).

$$
\begin{gathered}
\frac{d C_{x}}{d t}=\mu \cdot C_{x}-\frac{F}{V}(1-R) \cdot C_{x} \\
\frac{d C_{s}}{d t}=\frac{F}{V}\left(C_{s, i n}-C_{s}\right)-q_{s} \cdot C_{x} \\
q_{s}=\frac{\mu}{Y_{x / s}^{\max }}+m_{s} \\
Y_{x / s}^{\max }=Y_{x / A T P}^{\max } \cdot Y_{A T P / s} \\
m_{s}=\frac{m_{A T P}}{Y_{A T P / s}}
\end{gathered}
$$

Because the lactose concentration in the bioreactor was much lower than the concentration in the medium and citrate was not consumed at all, a steady state can be assumed, i.e. $\mathrm{dC}_{\mathrm{s}} / \mathrm{dt}=0$. After substitution of equation 9.4 in equation 9.3, an expression for $\mu$ can be obtained, which can be substituted in equation 9.2. Integration of this equation and substitution of $Y_{x / s}^{\max }$ and $\mathrm{m}_{\mathrm{s}}$ using equation 9.5 and 9.6 leads to equation 9.7:

$$
C_{X}(t)=\left(C_{x, 0}-\frac{\left(\frac{F}{V} \cdot\left(C_{s, i n}-C_{S}\right) \cdot Y_{X / A T P}^{\max } \cdot Y_{A T P / s}\right)}{Y_{x / A T P}^{\max } \cdot m_{A T P}-(1-R) \cdot \frac{F}{V}}\right) \cdot e^{-\left(Y_{x / A T P}^{\max } \cdot m_{A T P}+(1-R) \cdot \frac{\mathrm{F}}{\mathrm{V}}\right) \cdot t}+\frac{\left(\frac{F}{\bar{V}} \cdot\left(C_{s, i n}-C_{S}\right) \cdot Y_{x / A T P}^{\max } \cdot Y_{A T P / s}\right)}{Y_{x / A T P}^{\max } \cdot m_{A T P}-(1-R) \cdot \frac{F}{V}}
$$

Equation 9.7 was used to estimate the energy-related maintenance coefficient $\mathrm{m}_{\text {ATP }}$ in time by fitting the biomass accumulation, assuming a constant $\mathrm{Y}_{\mathrm{X} / \mathrm{ATP}}{ }^{\max }$ of $15.94 \mathrm{gDW} / \mathrm{mol}$ ATP, as determined for this strain in chemostat cultures at growth rates between 0.05 and $0.4 \mathrm{~h}^{-1}$ (van Mastrigt et al., 2018a). The $\mathrm{Y}_{\text {ATP/s }}$ was estimated based on the measured metabolite production:

$$
Y_{A T P / S}=\frac{R_{\text {lactate }}+R_{\text {ethanol }}+R_{\text {pyruvate }}+2 \cdot R_{\text {acetoin }}+2 \cdot R_{\text {acetate }}}{-\left(12 \cdot R_{\text {lactose }}\right)}
$$

in which $R_{i}$ is the production rate ( $\mathrm{mol} / \mathrm{h}$ ) of compound $\mathrm{i}$. Input data for the fitting were the online optical density measurements, which were first converted into biomass dry weight concentrations using a second-order polynomial relation (Suppl. Fig. S9.1). 
For fitting the data to equation 9.7, two different models were used, both describing a maintenance coefficient that decreases at near-zero growth rates as found by van Mastrigt et al. (2018a): i) a model in which the maintenance starts to decrease at growth rates below a critical growth rate $\left(\mu_{\text {cirt }}\right.$ (Eq. 9.9) and ii) a model in which the maintenance coefficient gradually decreases towards nearzero growth rates (Eq. 9.10).

$$
\begin{gathered}
m_{A T P}= \begin{cases}m_{A T P, \text { che }} & \text { if } \mu \geq \mu_{\text {crit }} \\
m_{A T P, \text { che }}-a \cdot\left(\mu_{\text {crit }}-\mu\right) & \text { if } \mu<\mu_{\text {crit }}\end{cases} \\
m_{A T P}=m_{A T P, \text { che }}-b \cdot e^{-c \cdot \mu}
\end{gathered}
$$

in which $m_{A T P}$, che denotes the $m_{\text {ATP }}$ as found by extrapolation from chemostat cultures $\left(2.43 \mathrm{mmol}\right.$ ATP.gDW $\left.{ }^{-1} . \mathrm{h}^{-1}\right), \mu_{\text {crit }}$ is the critical growth rate from where the maintenance coefficient starts to decrease and $a, b$ and $c$ are fitted parameters describing how fast the maintenance coefficient decreases. For both models, we minimised the sum of squared errors between the model and the input data by optimising either i) $\mu_{\text {crit }}$ and $a$ or ii) $b$ and $c$. This was done using the solver addin of Excel with 10 minute intervals.

\section{Results}

Lactococcus lactis FM03-V2 was cultivated in a partial cell recycling chemostat bioreactor with a constant dilution rate $\left(0.025 \mathrm{~h}^{-1}\right)$ at various recycling ratios (0 to 0.9 ) resulting in growth rates between 0.025 and $0.0025 \mathrm{~h}^{-1}$. The biomass concentration increased 3.4-fold at higher recycle ratios (Fig. 9.1). Throughout the cultivation, the viability remained high (>99\%) as determined by live/dead staining with the fluorescent markers SYTO 9 and propidium iodide.

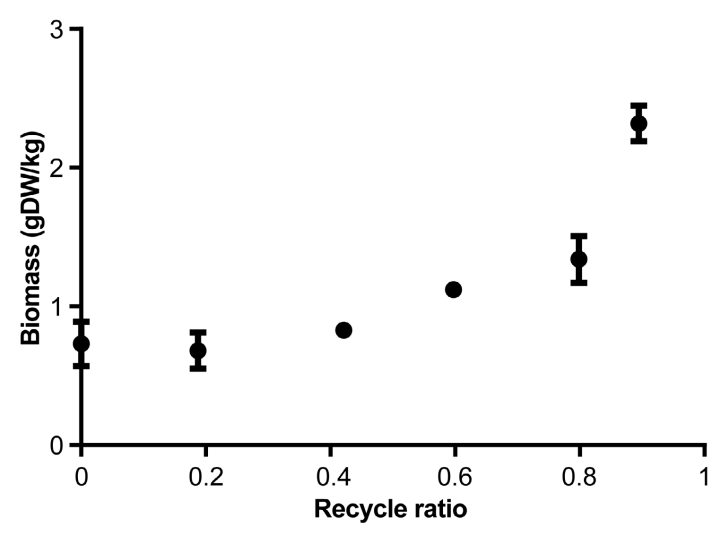

Figure 9.1: Biomass concentration in steady states of a partial cell recycling chemostat culture at different recycle ratios. Error bars represent the standard deviation of technical triplicates. 


\section{Metabolism}

The main carbon sources (lactose and citrate) and the main fermentation products (lactate, acetate, ethanol, formate, acetoin and pyruvate) were quantified in all steady states at growth rates between 0.025 and $0.0025 \mathrm{~h}^{-1}$ using HPLC (Fig. 9.2). Residual lactose concentrations were below the detection limit, while citrate was not consumed throughout the cultivation because $L$. lactis FM03-V2 does not carry plasmid pLd1 carrying the citQRP operon required for citrate uptake. The lactose was converted mainly into lactate, acetate, formate, ethanol and pyruvate. At lower growth rates, lactate production increased (from 53\% to 63\%), while the formation of acetate, ethanol and formate decreased (from $47 \%$ to $36 \%$ ). Furthermore, based on the production of acetate, ethanol and formate, it can be concluded that the pyruvate was converted into acetyl-CoA via both pyruvate formate lyase (PFL) and pyruvate dehydrogenase (PDH).

\section{Maintenance estimation}

Traditionally, the correlation between the biomass specific ATP production rate ( $q_{A T P}$ ) and the growth rate $(\mu)$ in chemostat cultures is used to determine the maximum biomass specific yield on ATP ( $\left.\mathrm{Y}_{\mathrm{X} / \text { ATP }^{\mathrm{max}}}\right)$ and the maintenance coefficient $\left(\mathrm{m}_{\text {ATP }}\right)$. However, this approach only takes into account growth rates above

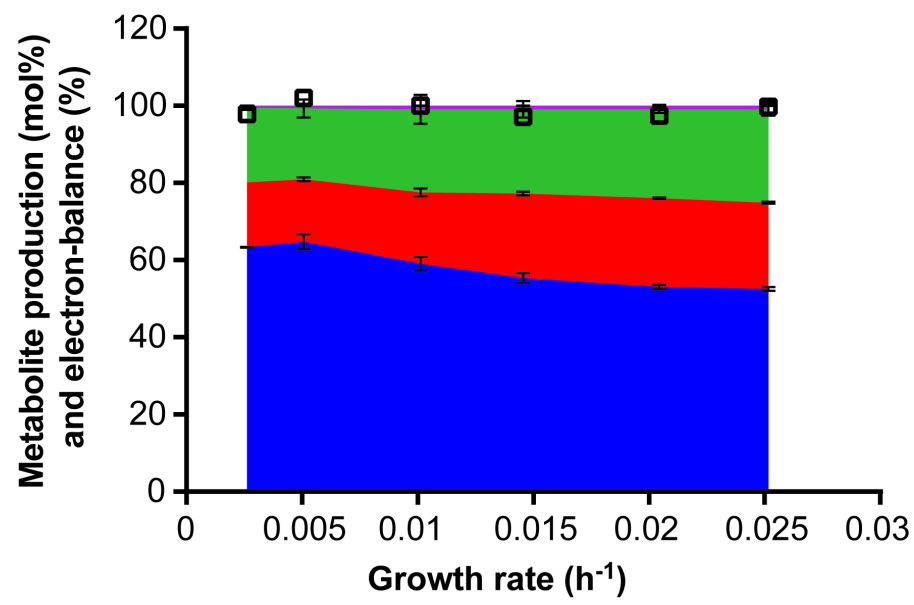

Figure 9.2: Metabolite production by L. lactis FM03-V2 in a partial cell recycling chemostat culture as function of the growth rate. The distribution of pyruvate over four different metabolic reactions was normalized to $100 \%$. Every colour corresponds to a particular reaction: lactate dehydrogenase (blue), pyruvate formate lyase (orange), pyruvate dehydrogenase (green), pyruvate efflux (purple). Reactions were normalized based on the production or consumption of 1 pyruvate or 1 acetyl-CoA. Squares represent the degree of reduction balance. Error bars represent the standard deviation of technical duplicates. 
approximately $0.05 \mathrm{~h}^{-1}$ and therefore deviations at low growth rates will not be found. Based on dynamic modelling of retentostat cultures, it was found that the $\mathrm{m}_{\mathrm{ATP}}$ of $L$. lactis FM03-V1 decreases 7-fold at near-zero growth rates (van Mastrigt et al., 2018a). We used the biomass concentrations and the metabolite production rates in the steady states of the partial cell recycling chemostat culture to calculate $\mathrm{q}_{\text {ATP }}$, which was plotted against the growth rate (Fig. 9.3). We calculated the ATP production rate $\left(\mathrm{R}_{\mathrm{ATP}}\right)$ using equation 9.11:

$$
R_{\text {ATP }}=R_{\text {lactate }}+R_{\text {ethanol }}+R_{\text {pyruvate }}+2 \cdot R_{\text {acetoin }}+2 \cdot R_{\text {acetate }}
$$

in which $R_{i}$ is the production rate of compound $i$. The $q_{\text {ATP }}$ was significantly lower at near-zero growth rates than estimations based on extrapolations of chemostat cultures at growth rates between 0.05 and $0.4 \mathrm{~h}^{-1}$ (3.6-fold at $0.0025 \mathrm{~h}^{-1}$ ) confirming that the maintenance coefficient decreased at near-zero growth rates. Moreover, the extent of the decrease in the maintenance coefficient seemed to increase towards near-zero growth rates. To further study the relation between the maintenance and the growth rate, the biomass accumulation in the partial cell recycling chemostat was modelled assuming that the maintenance coefficient decreased either linearly or exponentially at low growth rates as explained in the materials and methods (Eq. 9.9 and 9.10).

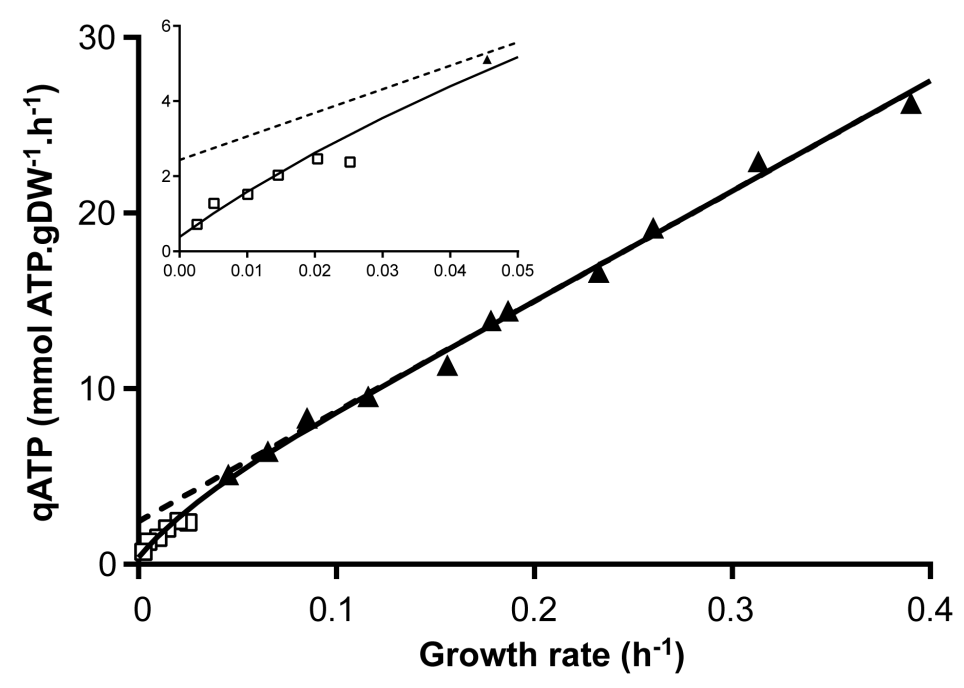

Figure 9.3: Relation between biomass specific ATP production rate ( $q_{A T P}$ ) and the growth rate based on chemostat cultures (filled triangles) and partial cell recycling chemostat cultures (open squares). The dashed line is a linear regression line based on data from chemostat cultures that is traditionally used to estimate the maximum biomass yield on ATP ( -1 /slope) and the maintenance coefficient (intercept). The black line represent the model in which the maintenance exponentially decreased at low growth rates. The axis of the inset are the same as of the main figure. 


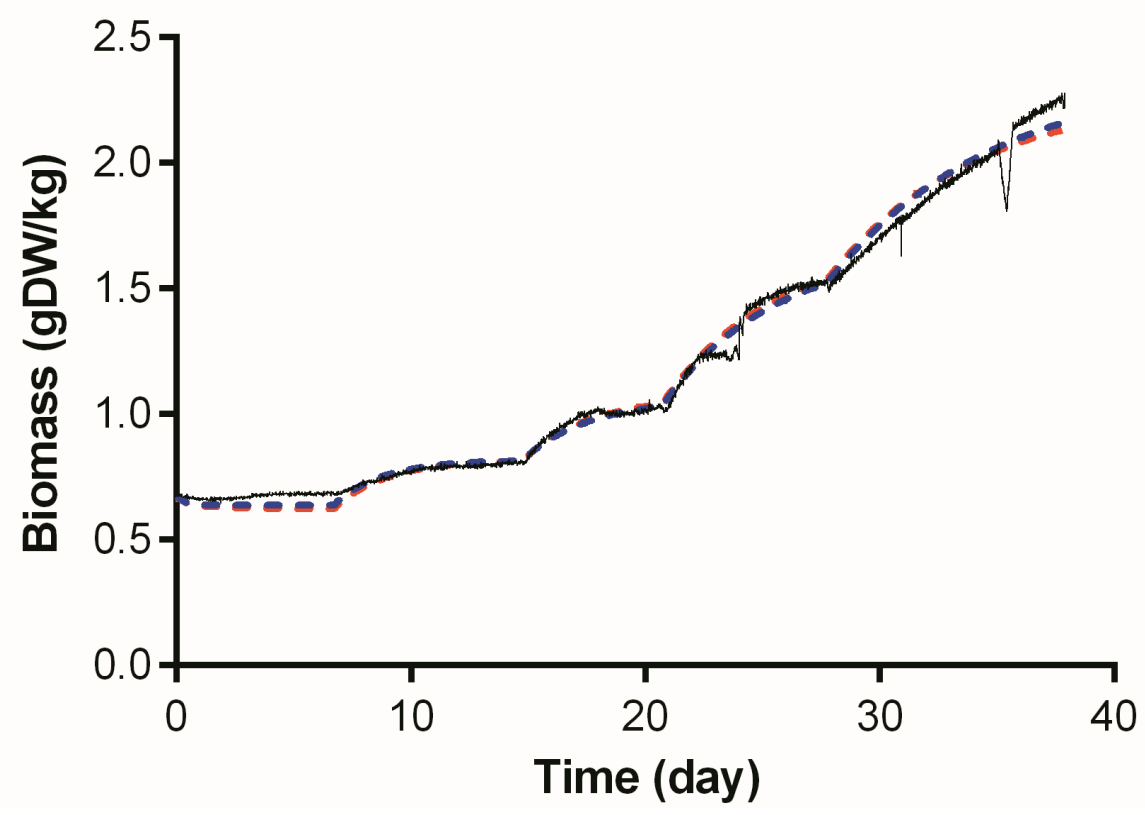

Figure 9.4: Prediction of the biomass accumulation of the partial cell recycling chemostat based on a linearly decreasing (red) or an exponentially decreasing maintenance coefficient towards lower growth rates (blue). The black line represent the optical density measurements at $600 \mathrm{~nm}$, which were converted to dry weight concentrations with a second-order polynomial function (Suppl. Fig. S9.1).

The model with an exponential decreasing maintenance coefficient fitted the data slightly better (root mean squared error (RMSE) $=0.035$ and $0.041 \mathrm{gDW} / \mathrm{kg}$ ) (Fig. 9.4). Moreover, in contrast to the linear model that predicts a sudden decrease, the exponential model predicts a gradual decrease in the maintenance requirements, which better reflects the microbial physiology. Therefore, the exponentially decreasing model is the preferred model. This model is in line with the traditional determination of the biomass yield and maintenance coefficient using conventional chemostat cultures (Fig. 9.3). Extrapolations of the traditional model with a constant maintenance coefficient and the new model with an exponentially decreasing maintenance coefficient showed that the maintenance coefficient decreases approximately 6.4 -fold when the growth rate decreases to zero.

\section{Volatile organic compound production}

To identify changes in the metabolism resulting from the low growth rates, volatile organic compounds were analysed in the steady states by headspace solid phase microextraction gas chromatography mass spectrometry. To be able to estimate 
the production rates, both complete and cell-free samples were analysed because it is known that particular compounds are predominantly located in the cell membrane due to their hydrophobic nature (van Mastrigt et al., 2018a). In total, 27 compounds were identified in the samples. Based on the flow rates of the effluent (corresponding to the complete samples) and the permeate (corresponding to cell-free samples) combined with the determined abundances of the metabolites in both samples, we calculated the production rates of each compound (Fig. 9.5). In general, the production of ketones, fatty acids and longchain alcohols increased towards low growth rates (i.e. acetone, 2-butanone, butanoic acid, hexanoic acid and, octanoic acid, 1-tetradecanol and 1-hexadecanol), while aldehydes, acetoin and pyrazines decreased (i.e. acetaldehyde, benzaldehyde, benzeneacetaldehyde, 2-butenal, 3-hydroxy-2butanone, trimethylpyrazine and 2,6-dimethylpyrazine). An increase of ketones and fatty acids at lower growth rates was also found for L. lactis FM03-V1 during retentostat cultivation (van Mastrigt et al., 2018a).

\section{Discussion}

Partial cell recycling chemostat cultivation was found to be a promising technique because higher biomass and metabolites production rates can be obtained compared to batch and chemostat cultures, in particular for diluted or insoluble substrates or to prevent product inhibition (Bibal et al., 1991, Bull and Young, 1981). For the first time, we showed that this system could also be used to cultivate microorganisms in a constant chemical environment at very low growth rates to continuously produce metabolites typical for low growth rates and to study the physiology of the microorganisms.

L. lactis FM03-V2 was cultivated at growth rates between 0.0025 and $0.025 \mathrm{~h}^{-1}$ in a partial cell recycling chemostat and secondary metabolite production was quantified with HS SPME GC-MS. In general, acids and ketones increased at lower growth rates, while aldehydes, acetoin and pyrazines decreased. Ketones and acids are groups that contain important aroma compounds in fermented dairy products, like cheese (Curioni and Bosset, 2002). Production of most of these compounds is related to the lipolytic activity of moulds, but our study shows that they can also be produced by L. lactis FM03-V2 at extremely low growth rates.

During the cultivation, $L$. lactis converted lactose mainly into lactate, acetate, ethanol, formate and pyruvate. It is known that $L$. lactis shifts from homolactic fermentation at high growth rates to the more energy efficient mixed-acid fermentation (combination of acetate, formate and ethanol) at low growth rates (Goel et al., 2015, Thomas et al., 1979). This shift has also been observed for 


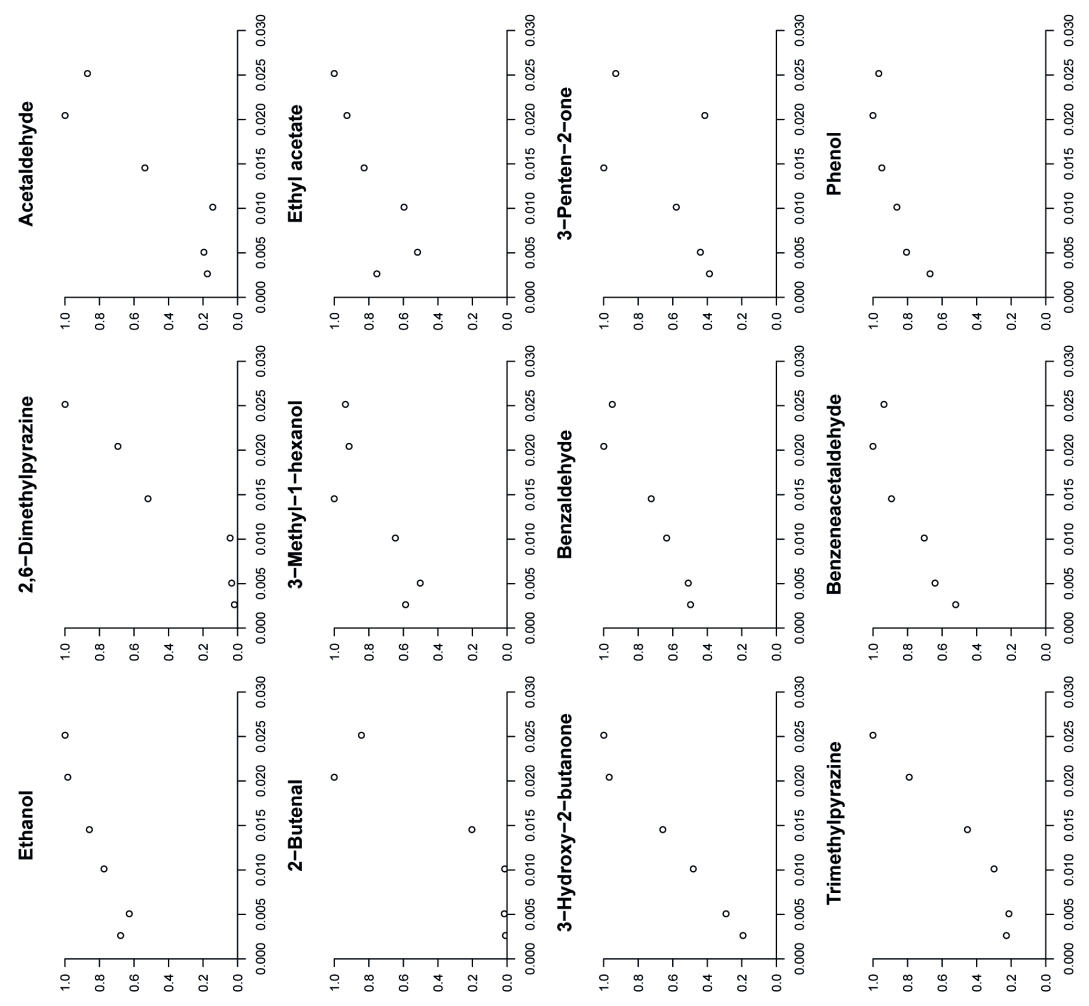

交

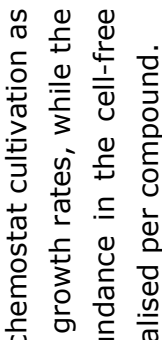

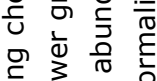

든 으웓



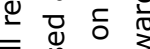

ब



离竞

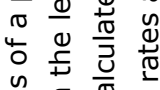

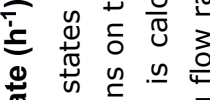

ᄂ

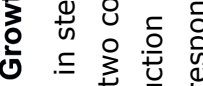
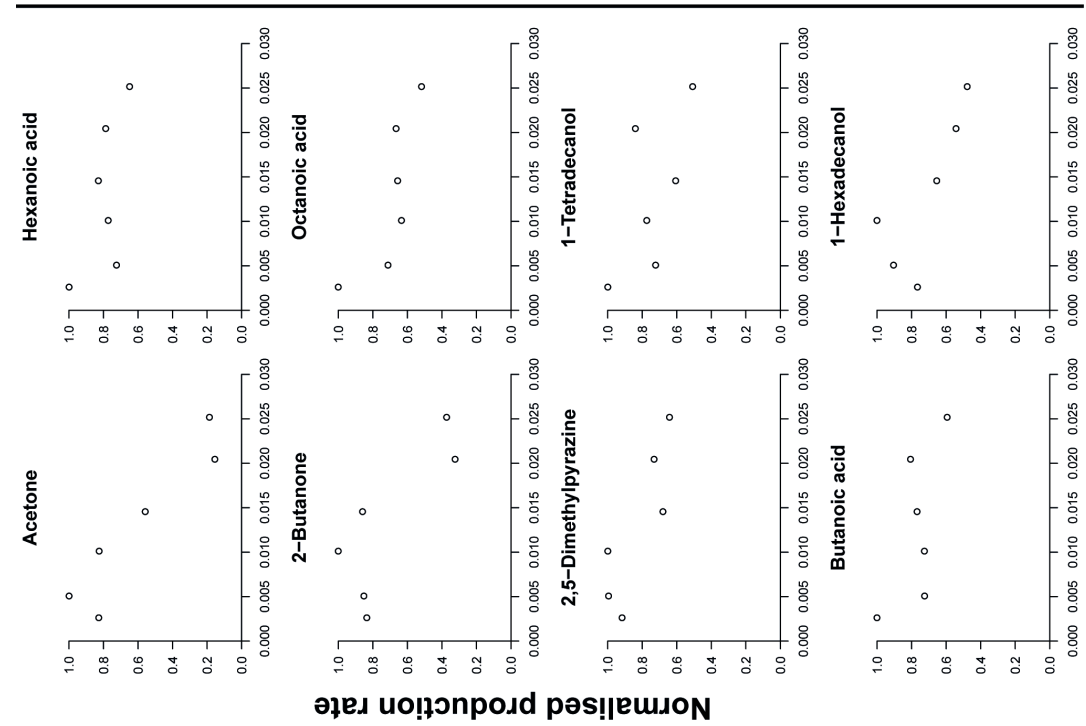

>

O들

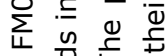

:

\&

ㄷำ

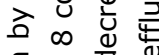

은 든 늠

몽

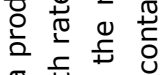

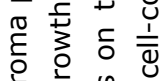

눈

ที่

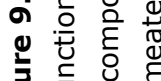
ปั่

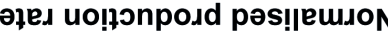


variants of L. lactis FM03-V1 (Suppl. Fig. S2). However, lactate production by $L$. lactis FM03-V2 increased at near-zero growth rates in the partial cell recycling chemostat. Why $L$. lactis switches between homolactic and mixed-acid fermentation remains unclear until now and these observations could contribute to answering this question (Goel et al., 2015, Goel et al., 2012).

Based on the metabolite production rates and the biomass concentration in the steady states of the partial cell recycling chemostat, we estimated the maintenance coefficient of $L$. lactis FM03-V2. The estimated maintenance coefficient decreased 6.4-fold at near-zero growth rates compared to high growth rate $\left(>0.05 \mathrm{~h}^{-1}\right)$, which corresponds well with predictions using dynamic modelling of retentostat cultures (7-fold) (van Mastrigt et al., 2018a). An exponentially decreasing maintenance coefficient was included in a dynamic growth model that excellently predicted the accumulation of biomass in the partial cell recycling chemostat supporting our hypothesis of an exponentially decreasing maintenance coefficient.

This study demonstrates that partial cell recycling chemostat cultivation can be used to study microorganisms at extremely low growth rates in static conditions and has important advantages over retentostat cultivation with complete cell recycling. The growth rate can be controlled at values just above zero, which results in a higher viability ( $>99 \%$ instead of $\sim 80 \%$ ). Moreover, the partial cell recycling chemostat is more reproducible due to its time-independency and it is not affected by small disturbances by for instance sampling, clogging of the filter or changes in the feed rate. This makes the partial cell recycling chemostat more suitable for omics studies, such as transcriptomics, proteomics and metabolomics. Furthermore, it offers the unique opportunity to study the impact of cell density and quorum sensing-dependent behaviour at a constant growth rate under constant environmental conditions by only changing the feed rate and the recycle ratio.

In conclusion, partial cell recycling chemostat cultivation is a unique cultivation technique that has both promising applications in biotechnological research, food production processes and in academic research.

\section{Acknowledgement}

This work was supported by Arla Foods (Aarhus, Denmark). 


\section{Supplementary materials}

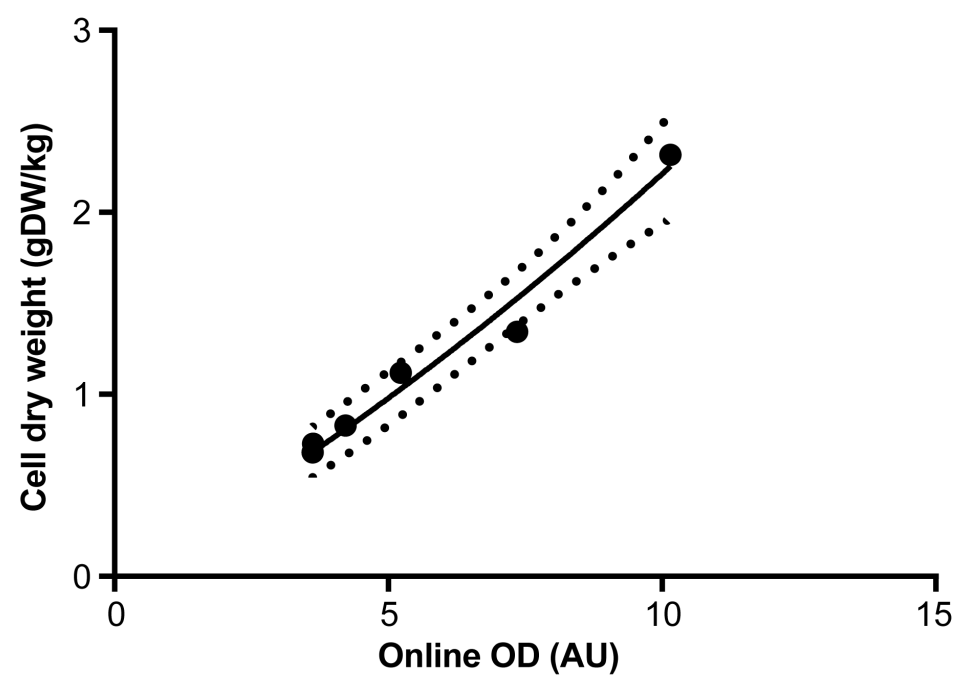

Supplementary Figure S9.1: Relation between the optical density at $600 \mathrm{~nm}$, measured continuously with an internal probe, and the biomass concentration. The solid line represents a second-order polynomial function and the dotted lines represent the $95 \%$ confidence interval.

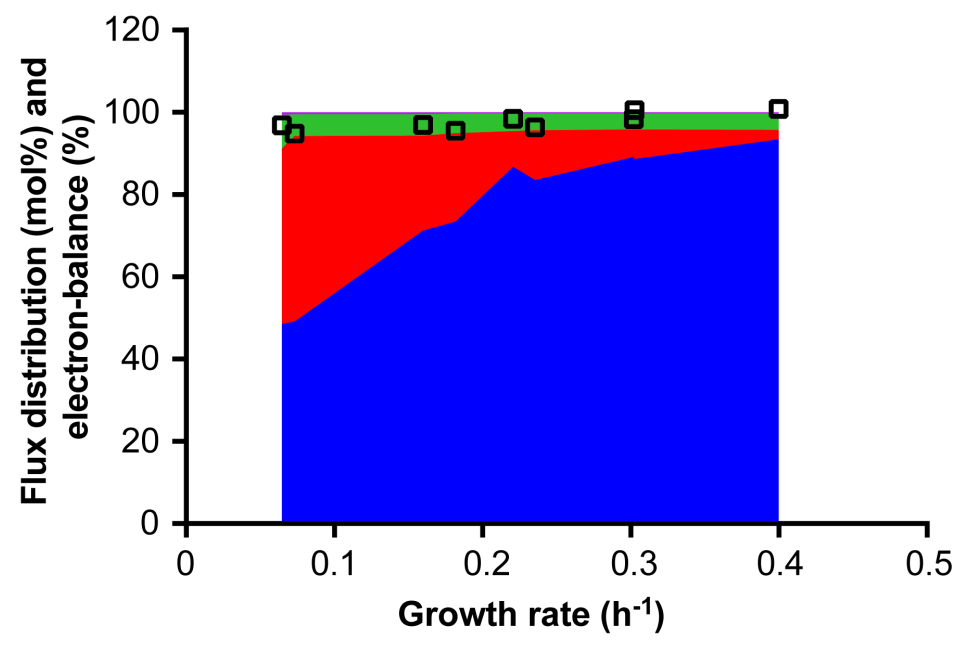

Supplementary Figure S9.2: L. lactis FM03-V1 shifted towards more mixed-acid fermentation at low growth rates in chemostat cultures. The distribution of pyruvate over four different metabolic reactions was normalised to $100 \%$. Every colour corresponds to a particular reaction: lactate dehydrogenase (blue), pyruvate formate lyase (red), pyruvate dehydrogenase (green), pyruvate efflux (purple). Reactions were normalised based on the production or consumption of 1 pyruvate or 1 acetyl-CoA. Squares represent the degree of reduction balance. 


\section{References}

Bibal, B., Vayssier, Y., Goma, G. and Pareilleux, A. (1991). High-concentration cultivation of Lactococcus cremoris in a cell-recycle reactor. Biotechnol. Bioeng. 37(8), 746-754.

Boender, L. G. M., de Hulster, E. A. F., van Maris, A. J. A., Daran-Lapujade, P. A. S. and Pronk, J. T. (2009). Quantitative physiology of Saccharomyces cerevisiae at nearzero specific growth rates. Appl. Environ. Microbiol. 75(17), 5607-5614.

Bull, D. N. and Young, M. D. (1981). Enhanced product formation in continuous fermentations with microbial cell recycle. Biotechnol. Bioeng. 23(2), 373-389.

Curioni, P. M. G. and Bosset, J. O. (2002). Key odorants in various cheese types as determined by gas chromatography-olfactometry. Int. Dairy J. 12(12), 959-984.

Ercan, O., Smid, E. J. and Kleerebezem, M. (2013). Quantitative physiology of Lactococcus lactis at extreme low-growth rates. Environ. Microbiol. 15(8), 2319-2332.

Goel, A., Eckhardt, T. H., Puri, P., de Jong, A., Branco dos Santos, F., Giera, M., Fusetti, F., de Vos, W. M., Kok, J., Poolman, B., Molenaar, D., Kuipers, O. P. and Teusink, B. (2015). Protein costs do not explain evolution of metabolic strategies and regulation of ribosomal content: does protein investment explain an anaerobic bacterial Crabtree effect? Mol. Microbiol. 97(1), 77-92.

Goel, A., Wortel, M. T., Molenaar, D. and Teusink, B. (2012). Metabolic shifts: a fitness perspective for microbial cell factories. Biotechnol. Lett. 34(12), 2147-2160.

Goffin, P., van de Bunt, B., Giovane, M., Leveau, J. H. J., Höppener-Ogawa, S., Teusink, B. and Hugenholtz, J. (2010). Understanding the physiology of Lactobacillus plantarum at zero growth. Mol. Syst. Biol. 6(413).

Herbert, D. (1961). A theoretical analysis of continuous culture systems. Paper presented at the Continuous Culture, London.

Overkamp, W., Ercan, O., Herber, M., van Maris, A. J. A., Kleerebezem, M. and Kuipers, O. P. (2015). Physiological and cell morphology adaptation of Bacillus subtilis at nearzero specific growth rates: a transcriptome analysis. Environ. Microbiol. 17(2), 346363.

Panikov, N. S., Mandalakis, M., Dai, S., Mulcahy, L. R., Fowle, W., Garrett, W. S. and Karger, B. L. (2015). Near-zero growth kinetics of Pseudomonas putida deduced from proteomic analysis. Environ. Microbiol. 17(1), 215-228.

Pirt, S. J. (1975). Principles of microbe and cell cultivation. Oxford: Blackwell.

Pirt, S. J. and Kurowski, W. M. (1970). An extension of the theory of the chemostat with feedback of organisms. Its experimental realization with a yeast culture. Microbiology 63(3), 357-366.

Thomas, T. D., Ellwood, D. C. and Longyear, V. M. C. (1979). Change from homo-to heterolactic fermentation by Streptococcus lactis resulting from glucose limitation in anaerobic chemostat cultures. J. Bacteriol. 138(1), 109-117.

van Mastrigt, O., Abee, T., Lillevang, S. K. and Smid, E. J. (2018a). Quantitative physiology and aroma formation of a dairy Lactococcus lactis at near-zero growth rates. Food Microbiol. 73, 216-226.

van Mastrigt, O., Mager, E. E., Jamin, C., Abee, T. and Smid, E. J. (2018b). Citrate, low pH and amino acid limitation induce citrate utilisation in Lactococcus lactis biovar diacetylactis. Microb. Biotechnol. 11(2), 369-380. 



\section{Chapter 10}

General discussion 


\section{General discussion}

Cheese ripening is a complex process in which the typical cheese characteristics, such as the flavour and texture, are formed by the action of numerous enzymes derived from the milk, the rennet, the starter bacteria and the non-starter bacteria (Fox et al., 2017). It is a slow process, and thus expensive, that can last from about two weeks, e.g. Mozzarella, to more than two years, e.g. ParmigianoReggiano (McSweeney, 2004). Ripening costs include the need to keep the temperature low to prevent growth of spoilage and pathogenic microorganisms and, often, to control the relative humidity (McSweeney, 2007). Cheese ripening primarily involves the generation of precursors from carbohydrates, proteins and fats and their conversion into a wide range of compounds responsible for the flavour and texture of the cheese. While precursors can be formed by single enzymes, the subsequent aroma formation required intact cells and functional metabolic pathways (Smid and Kleerebezem, 2014).

During cheese ripening, nutrients are limited. Most of the lactose, the main energy source in milk, has been discarded in the whey fraction or is already consumed by the bacteria during the early stages of cheese making. Therefore, lactic acid bacteria $(L A B)$ encounter long periods of nutrient limitation during cheese ripening leading to severe reduction of the growth rate. Particular $L A B$, such as Lactococcus lactis biovar diacetylactis and Leuconostoc mesenteroides, survive these periods of extremely slow growth (Erkus et al., 2013), while still contributing to flavour formation in the fermented product.

Such low nutrient availabilities are not only found in food fermentation processes, but are often found in natural environments, in which microorganisms generally live in a feast or famine existence due to variable availabilities of nutrient and energy sources (Koch, 1971, Morita, 1993). It has been suggested that energy limitation is the prevailing physiological state among microorganisms on Earth (Brock, 1971, Morita, 1997). The physiological state of these organisms is poorly represented in laboratory experiments with nutrient-rich batch cultures, in which metabolic rates are several orders of magnitude higher than in oligotrophic environments (Hoehler and Jorgensen, 2013). The high prevalence of slowly growing microorganisms in both food fermentation processes and natural environments demonstrates that its very relevant to study microorganisms that are hardly growing.

In this study, dairy isolates of lactic acid bacteria were grown in retentostat cultures at near-zero growth rates to study their physiological, genetic and metabolic adaptations to these harsh conditions (Chapters 5, 6, 7 and 8). In the 
following paragraphs, these adaptations will be mainly discussed from the perspective of the microbe and its long evolution in the dairy environment.

\section{Physiological adaptations}

In retentostat cultures of $L$. lactis biovar diacetylactis FM03-V1 and $L C$. mesenteroides FM06 growth rates decreased to less than $0.001 \mathrm{~h}^{-1}$, corresponding to doubling times of more than a month (Chapter 6 and 7). Both strains showed very similar physiological responses towards the long periods of nutrient limitation, such as that a large fraction of the cells lost the ability to grow on plates, while remaining viable, i.e. have an intact cell membrane. These so-called viable but non-culturable (VBNC) cells have entered a dormant state, which is an important survival strategy against unfavourable environmental conditions. For instance, the fraction of dormant cells in soil samples can be more than $80 \%$ (Lennon and Jones, 2011). VBNC cells have large similarities with persisters, which are antibiotic tolerant but not growing, and can be formed stochastically owing to random fluctuations in gene expression or due to a wide range of stresses, such as starvation, growth-inhibiting temperatures, non-optimal salinity and stressful pH (Ayrapetyan et al., 2015, Maisonneuve et al., 2013). Our results demonstrate that near-zero growth rates induce the formation of VBNC cells. In Escherichia coli, near-zero growth rates coincide with increased levels of ppGpp (Arbige and Chesbro, 1982), which is thought to be the inducer of the VBNC state (Ayrapetyan et al., 2015). Theory predicts that the benefits of dormancy are greater in environments with low resource availability because of the lower costs of entering this state when the growth rates are low anyway (Gardner et al., 2007). This is in line with the observed increase in VBNC cells during retentostat cultivations. Retentostat cultivation also induced a viable but non-culturable state in Pseudomonas putida (Panikov et al., 2015), indicating that this is a general phenomenon and not specific for the dairy isolates in this study. Finally, also during cheese ripening many L. lactis cells enter a VBNC state (Erkus et al., 2013, Erkus et al., 2016, Ruggirello et al., 2016), indicating that retentostat cultivation mimics the conditions during cheese ripening.

Another physiological adaption of $L$. lactis biovar diacetylactis FM03-V1 and LC. mesenteroides FM06 towards near-zero growth rates was the 7-fold decrease in the maintenance requirements, which was initially found using dynamic modelling of the biomass accumulation in retentostat cultures (Chapters 6 and 7) and later confirmed for $L$. lactis using a partial cell recycling chemostat cultivation at growth rates between 0.0025 and $0.025 \mathrm{~h}^{-1}$ (Chapter 9). This latter experiment showed that the maintenance costs could be well predicted with an exponentially 
decreasing maintenance coefficient towards lower growth rates. Kempes et al. (2017) estimated that the protein turnover would be the main maintenance cost for L. lactis, which was confirmed by Lahtvee et al. (2014). Moreover, they showed that protein turnover rates decrease at lower growth rates in L. lactis, suggesting that the reduced maintenance requirements of both dairy isolates was due to a lower protein turnover rate. This is in line with the reduced expression of the translational machinery during retentostat cultivation in Bacillus subtilis and Saccharomyces cerevisiae (Boender et al., 2011, Overkamp et al., 2015).

During retentostat cultivation of L. lactis KF147, expression of the $y$ fiA, encoding the hibernation promotion factor (Franken et al., 2017, Puri et al., 2014), increased causing ribosome dimerisation (Fig. 10.1). The ribosome dimers are translationally inactive, and dimerisation protects ribosomal ribonucleic acids (rRNAs) against degradation by ribonucleases.

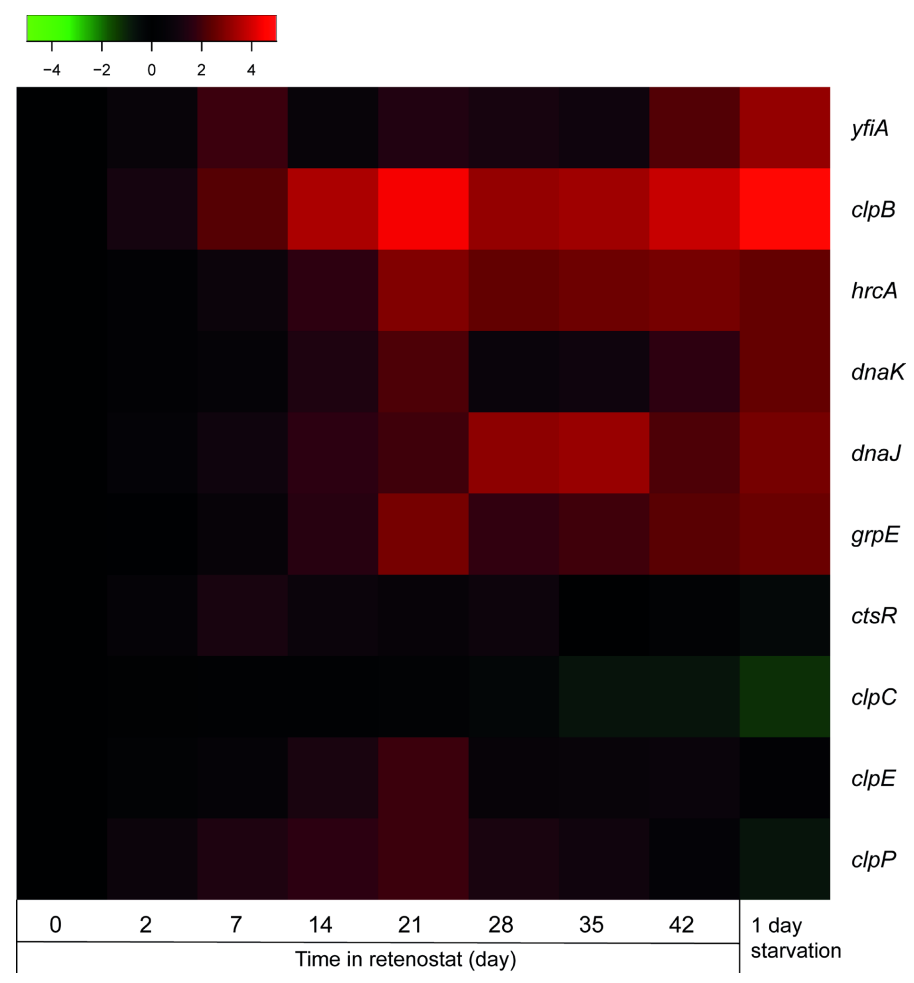

Figure 10.1: Expression of genes related to protein turnover (e.g. protein synthesis, disaggregation, (re)folding, degradation) during retentostat cultivation of L. lactis KF147 and subsequent starvation for 1 day. Colours represent the relative expression compared to the start of the retentostat (day 0 ) on a $\log _{2}$-scale. Transcriptomic data of retentostat cultures of $L$. lactis KF147 were taken from the NCBI Gene Expression Omnibus (GEO) (Ercan et al., 2015). 
Interestingly, the gene $c / p B$, encoding an ATP-dependent protein disaggregase belonging to the Hsp100 family of ATPases associated with diverse cellular activities (AAA+) (Parsell et al., 1994, Schirmer et al., 1996), was strongly induced (>10-fold) during retentostat cultivation of L. lactis KF147 (Fig. 10.1) and also genes encoding chaperones DnaK, DnaJ and GrpE that cooperate with ClpB in protein disaggregation (Zolkiewski, 1999) were highly induced ( 5 to 10 -fold).In contrast, genes encoding the chaperone GroESL and proteases ClpC, ClpE and ClpP were not or less highly induced (<4-fold) (Fig. 10.1). The difference in relative expression was even more distinct after 1 day of starvation ( $>5$-fold and $<1.2$-fold, respectively). The differences in expression patterns could be attributed to the transcriptional repressors regulating these genes. While genes groES, groEL, $c l p C, c l p E, c l p P$ are regulated by $C t s R$, the genes encoding chaperones DnaK, DnaJ and GrpE are regulated by HrcA (Sugimoto et al., 2008). Interestingly, in the $c / p B$ promoter region of $L$. lactis only an CtsR-box has been found, but the different expression pattern in the retentostat cultures compared to the other CtsR-regulated genes suggests that induction of $c / p B$ at near-zero growth rates was caused by another factor. Identifying this factor could help to further decipher the mechanisms by which L. lactis copes with extreme nutrient limitation.

The mechanism of the protein disaggregation machinery was recently reviewed by Mogk et al. (2018). They suggested that after protein disaggregation, refolding is favoured over proteolysis saving cellular resources (Mogk et al., 2018). Moreover, they suggested that this system can become essential for cell growth upon heat-induced aggregation when central factors of transcription and translation machineries are depleted (Mogk et al., 2018). Retentostat studies with L. lactis KF147 showed that this system might also be essential at near-zero growth rates that caused repression of the translational machinery. Lowering the translational machinery while increasing protein disaggregation and refolding could be beneficial during long periods of nutrient limitation, but this bet-hedging technique could be disadvantageous in fluctuating environments that require synthesis of additional proteins (Fig 10.2).

Interestingly, under normal growth conditions ( $\left.\mu>0.05 \mathrm{~h}^{-1}\right)$ the maintenance coefficient of dairy strains of $L$. lactis, including FM03-V1, is very high compared to that of the plant-associated L. lactis KF147, while the maximum yield of biomass on substrate is low (Chapter 6). Dairy strains have a long history of being propagated in the dairy environment through backslopping. Because lactose is abundant throughout this backslopping procedure, growth of the bacteria was most likely not energy-limited, but rather limited by the rate of the biosynthetic routes. Thereby, the dairy environment selected for fast growers, which in general 




Figure 10.2: Putative model describing the management of protein turnover processes in Lactococcus lactis and the impact of the growth rate on these processes. The thickness of the arrows represents the conversion rates. Protein turnover is high at high growth rates and decreases at lower growth rates (Lahtvee et al., 2014). At near-zero growth rates, the strategy of the bacteria switches from the degradation and re-synthesis of misfolded proteins to disaggregation and refolding of misfolded proteins as indicated by the induction of the ClpB-DnaK system and the ribosome hibernation factor (Fig. 10.1). Thereby, valuable energy is saved. This response to energy limitation becomes even more evident during starvation conditions. 
do not utilise the nutrients efficiently (high maintenance costs and low biomass yield). Surprisingly, the dairy isolates were able to adapt to long periods of nutrient limitation by becoming more energy efficient in terms of the decreased maintenance costs. Compared to the dairy environment, the plant environment is poor in nutrients and selected for efficient use of the nutrients as indicated by the energy-efficient L. lactis KF147 (Ercan et al., 2013).

\section{Genetic adaptations}

Whole genome sequencing of L. lactis FM03P and LC. mesenteroides FM06 revealed that both strains were adapted to the dairy environment mainly via the acquisition of plasmids (Chapters 2 and 3). Both strains harboured multiple plasmids involved in lactose uptake and utilisation, bacteriophage resistance, heavy metal resistance and cation uptake (Fig. 10.3). Moreover, plasmids on $L$. lactis FM03P carried genes involved in citrate uptake, peptide degradation, oligopeptide uptake, exopolysaccharide production and stress resistance. Additionally, several novel genes for $L$. lactis were found on plasmids of $L$. lactis FM03P, including genes encoding a putative type IIG restriction-modification system with similarity to the recently identified Class I DISARM system for bacteriophage defence and a putative peptidoglycan-binding protein with unknown function. While $L$. lactis FM03P carries a whole lactose operon encoding a lactose phosphotransferase system (PTS) and all enzymes of the tagatose-6phosphate pathway on one plasmid, Lc. mesenteroides carries genes encoding a lactose permease (lacS) and a $\beta$-galactosidase (lacLM) on two different plasmids and lactose is metabolised via the Leloir pathway by enzymes encoded on the chromosome. Genes or remnants thereof encoding a putative lactose permease and a $\beta$-galactosidase were also found on the chromosome of $L c$. mesenteroides FM06. This suggests that acquisition of one of the plasmids, carrying either lacS or lacLM, could already have been beneficial for growth on lactose explaining the unusual arrangement.

Because the plasmids carry important genes for growth and survival as well as for flavour formation (e.g. citQRP operon), the stability of the plasmids is crucial when employing these lactic acid bacteria in industrial processes. This was clearly demonstrated by the loss of plasmid pLd1 in the variant that was used for the partial cell recycling chemostat (Chapter 9). Due to the loss of this plasmid, which occurred most likely already during pre-culturing in LM17 broth, this variant was unable to utilise citrate affecting both growth and aroma production. This is the first time that the spontaneous loss of citrate plasmid, and thus loss of the diacetylactis phenotype, has been described, according to the best of our 



Leuconostoc mesenteroides FM06

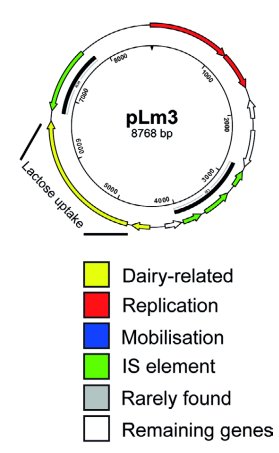

Figure 10.3: Genetic maps of the plasmidomes of Lactococcus lactis FM03P (top) and Leuconostoc mesenteroides FM06 (bottom). Important dairy functions encoded by the plasmids are indicated. 
knowledge. Several plasmids of L. lactis FM03P were spontaneously lost during propagation on M17 supplemented with lactose or chemically defined medium (Chapter 3), signifying their instability outside the dairy environment in the absence of a selection pressure. This showed that propagation in the laboratory should be drastically minimised when studying dairy isolates to avoid plasmid loss.

This thesis described the first study of the effect of near-zero growth rates on copy numbers of five plasmids of L. lactis FM03-V1. In retentostat cultures, the copy numbers of these five plasmids were remarkably stable even at near-zero growth rates (Chapter 8 ). These observations show that plasmid copy numbers are strictly regulated, but plasmids can be lost during cell division. This implies that high copy number plasmids are segregationally more stable than low copy number plasmids. During retentostat cultivation, cell divisions are limited and therefore a lower chance of plasmid loss during prolonged incubation is predicted. However, the extreme energy limitation in retentostat cultivations could select for the loss of plasmids that have a large metabolic burden, as found for plasmid pLd10 carrying a large exopolysaccharide gene cluster (Chapter 8). This demonstrates that when retentostat cultivation would be applied in an industrial setting, plasmid loss during prolonged cultivation has to be taken into account. In particular, when important genes for the production process are located on plasmids as illustrated by loss of pLd1 abolishing citrate uptake and reducing the production of acetoin and diacetyl (Chapter 9). One solution would be to limit the cultivation time and/or to monitor the process performance or the plasmid content during the cultivation to detect plasmid loss. Another possible solution is to use a strain in which all relevant functions are encoded by genes located on the chromosome or use preferably non-gmo methods to obtain such a strain.

\section{Metabolic adaptations}

Metabolic adaptations of $L$. lactis and $L C$. mesenteroides towards near-zero growth rates were studied by analysing the production of main metabolites (lactate, acetate, ethanol, formate and acetoin) and volatile secondary metabolites. It is known that the homofermentative $L$. lactis produces mainly lactate at high growth rates (homolactic fermentation) and this changes to production of acetate, ethanol and formate at low growth rates (mixed-acid fermentation) (Goel et al., 2015, Thomas et al., 1979). This shift might be the result of a trade-off between the metabolic or energetic efficiency of the pathways and the cost of synthesising the enzymes for the pathways (Molenaar et al., 2009). This shift was also found for L. lactis FM03-V1 in chemostat cultures at dilution rates between 0.025 and 0.6 $\mathrm{h}^{-1}$ (Chapter 9). However, at low growth rates approximately $50 \%$ of the carbon 
flux was still converted to lactate in contrast to other L. lactis strains that shift almost completely to mixed-acid fermentation (Goel et al., 2015). A sensitivity analysis performed on the mathematical model of Molenaar et al. (2009) suggests that this difference might be caused by different transport kinetics. Interestingly, lactate production slightly increased at even lower growth rates $\left(<0.025 \mathrm{~h}^{-1}\right)$ in a partial cell recycling chemostat culture (Chapter 9) and in retentostat cultures (Chapter 6), which could not be explained by the trade-off hypothesis.

Using headspace solid phase microextraction gas chromatography mass spectrometry, we identified in total more than 100 volatile organic compounds (Chapters 5, 6, 7 and 9). Aroma production by slow-growing L. lactis cells differed from fast-growing cells and was more similar to aroma production during cheese ripening (Chapter 5). Several products of amino acid and fat catabolism increased during retentostat cultivation resembling biochemical reactions occurring in cheese. In the following sections, explanations for the increase in aroma formation at near-zero growth rates are discussed shedding light on the ecological role of aroma formation in lactic acid bacteria.

Cell lysis is known to play an important role in aroma formation in cheese by delivering enzymes that generate the precursors for aroma formation from the proteins (Crow et al., 1995). However, the subsequent conversion into aroma compounds often requires intact cells and functional metabolic pathways (Smid and Kleerebezem, 2014). In the chemically defined media used for the retentostat cultivations, amino acids were supplied as small peptides, which can directly be taken up by the bacteria via their oligopeptides transporters and hydrolysed intracellular to amino acids. Therefore, cell lysis was not required to generate the precursors, i.e. hydrolyse the protein into peptides and amino acids, and was most likely not causing the observed changes in aroma formation in the retentostat cultures.

Another explanation could be that genes involved in aroma formation were induced at the near-zero growth rates. To investigate this possibility, transcriptomic data of retentostat cultures of L. lactis KF147 were taken from the NCBI Gene Expression Omnibus (GEO) (Ercan et al., 2015) and the expression of genes involved in flavour formation were analysed. Unfortunately, only genes encoding an acyl-ACP thioesterase ( $y$ IfFG in L. lactis KF147) and fatty acid-CoA synthase (LLKF_1293 in L. lactis KF147) have been identified in L. lactis, which are both involved in fatty acid degradation but not necessarily in conversion of fatty acids in aroma compounds that requires acyl-CoA thioesterase (encoded by fadM in E.coli) (Yu et al., 2014). Therefore, only flavour formation from amino acids was analysed. An overview of flavour formation from amino acids by lactic 
acid bacteria can be found in Chapter 1 . The expression of genes that are expected to positively contribute to flavour formation from amino acids remained unchanged or decreased during the retentostat cultivations compared to the start of the cultivations (Fig 10.4A) and the expression of panE, encoding a protein catalysing the formation of hydroxyacids from a-keto acids thereby reducing flavour formation, was increased. This indicates that the increase of aroma production from amino acids at near-zero growth rates could not be explained by the induction of the genes involved in amino acid catabolism, although posttranscriptional regulation cannot be ruled out.

A
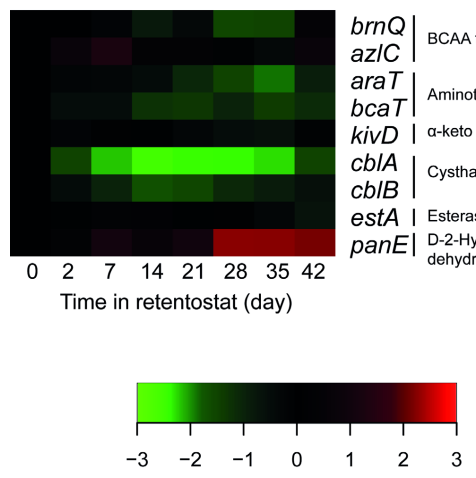

B

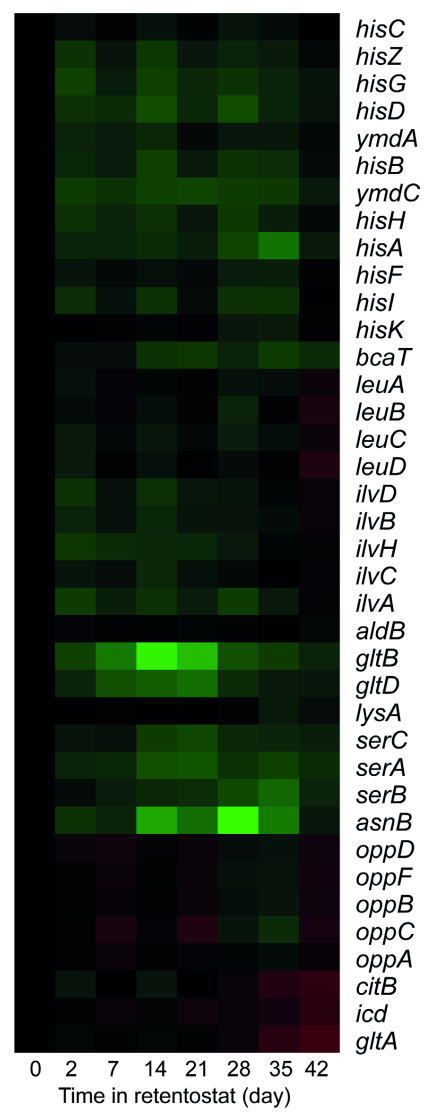

Figure 10.4: Expression of genes during retentostat cultivation of L. lactis KF147. A: Genes related flavour formation by amino acid catabolism of branched-chain amino acids, aromatic amino acids and methionine. B: Genes regulated by CodY. Colours represent the relative expression compared to the start of the retentostat (day 0 ) on a $\log _{2}$-scale. Transcriptomic data of retentostat cultures of L. lactis KF147 were taken from the NCBI Gene Expression Omnibus (GEO) (Ercan et al., 2015). 
Another explanation of the increase in aroma formation from amino acids and fatty acids could be an increased intracellular concentration of these precursors driving the catabolism. Intracellular metabolite concentrations have never been measured in retentostat cultures, but because regulation by the transcriptional repressor CodY is dependent on the intracellular branched-chain amino acid (BCAA) pool (Guédon et al., 2001), transcriptome data could give some insight. During retentostat cultivation of L. lactis KF147, CodY-regulated genes (den Hengst et al., 2005) were repressed (Fig. 10.4B), suggesting indeed an increased intracellular BCAA concentration. Fatty acids are known to induce the $\beta$-oxidation system both in L. lactis ( $\mathrm{Li}$ and $\mathrm{Ma}, 2013$ ) and in E. coli (Campbell et al., 2003). Therefore, increased fatty acids concentrations would most likely result in increased aroma production from the fatty acids.

The above considerations indicate that aroma production by L. lactis could be a mechanism to cope with high concentrations of branched-chain amino acids and fatty acids. Fatty acids are antimicrobial compounds that can inhibit microbial growth due to their amphipathic structure allowing them to interact with the cell membrane (Desbois and Smith, 2010). Thereby, they could creating transient pores and/or affect membrane-located processes, such as disruption of the electron transport chain, uncoupling of oxidative phosphorylation and inhibition of nutrient uptake (Desbois and Smith, 2010). The antimicrobial activity of fatty acids increases at low $\mathrm{pH}(\mathrm{pH}<\mathrm{pKa})$ when most of the acid is in the undissociated form (Lawrence and Hawke, 1968, Lewis and Darnall, 1970). By conversion into methyl ketones and alcohols, this growth inhibition can be relieved as also suggested for fungi (Kinderlerer, 1993). Although branched-chain amino acids are not harmful in nature, at high concentrations they could be growth inhibiting due to their role in the regulation of nitrogen metabolism by the transcriptional repressor CodY (Chambellon and Yvon, 2003). Chambellon and Yvon showed that simultaneous inactivation of AraT and BcaT, which are the main aminotransferases in L. lactis with activity towards branched-chain amino acids, strongly reduced growth in milk. When milk was supplemented with isoleucine or a dipeptide containing isoleucine, growth of the double mutant was even further inhibited. The excess of isoleucine most likely caused CodY-mediated repression of the proteolytic system, which is required for supply of casein-derived amino acids and peptides to support maximal growth of $L$. lactis in milk. The wild-type L. lactis was not affected by the excess of isoleucine due to catabolism of the isoleucine relieving CodY repression demonstrating that amino acid catabolism can act as mechanism to cope with high concentrations of branched-chain amino acids.

Aroma production as mechanism to cope with high concentrations of branchedchain amino acids and fatty acids does not fully explain why aroma production 
increases at near-zero growth rates during retentostat cultivation. During retentostat cultivation of $L$. lactis $\mathrm{KF147}$, the consumption of amino acids decreased during the first 21 days (Ercan et al., 2015), reflecting the decreased requirement of amino acids for protein synthesis at near-zero growth rates. This was most likely caused by CodY-repression of the amino acid transporters due to increased intracellular concentrations of branched-chain amino acids. However after 21 days, the consumption of amino acids suddenly increased, while the bacteria still grew at near-zero growth rates. Most likely these amino acids were converted in their corresponding aroma compounds. This shift coincides with the increase in viable but non-culturable cells (Ercan et al., 2013), indicating that they are part of the same response. This is strengthened by the observation that amino acid metabolism increased in $L$. lactis in viable but non-culturable cells after carbohydrate starvation (Ganesan et al., 2006, Stuart et al., 1999). With this response, the bacteria changed their strategy to prevent growth inhibition by the branched-chain amino acids from limiting the uptake and/or synthesis of amino acids to enhancing degradation of amino acids into aroma compounds.

In addition to its possible role as release valve for excess of branched-chain amino acids as described above, several other roles have been suggested for aroma production. These roles include redox balancing (Smit et al., 2005), detoxification (e.g. fatty acids are antimicrobial) (Kinderlerer, 1993), alkalisation of the intracellular $\mathrm{pH}$ (proton consumption in decarboxylation reactions), ATP generation (Chambellon et al., 2009, Ganesan et al., 2006) and providing essential compounds for sterol and branched-chain fatty acid synthesis (Oku and Kaneda, 1988). Moreover, the aroma compounds can act as growth-promotion or inhibition agents (Schmidt et al., 2015) or as communication signals between cells, between bacterial species or between inter kingdom species (bacteria, plant, fungi, host) (Audrain et al., 2015, Kai et al., 2009). Since dairy L. lactis strains are thought to have evolved from plant-associated strains (Cavanagh et al., 2015), in particular the interactions with plants could be interesting to study. Retentostat cultures of Lactobacillus plantarum, in which indole compounds were produced, inhibited the development of radish roots demonstrating, that $L b$. plantarum produces compounds at near-zero growth rates that interact with plant physiology (Goffin et al., 2010).

\section{Future perspectives}

This study demonstrates that the production of typical cheese aroma compounds by lactic acid bacteria increased at near-zero growth rates. Instead of milk, a chemically defined medium was used for retentostat cultivations because the 
cultures were continuously filtered and milk would quickly clog the filter. In Chapter 6 we described the development of hydrolysed micellar casein isolate (MCI), which is a new milk-based medium that could be used for retentostat cultivation after a cross-flow filtration step. This medium better resembles milk and the use of hydrolysed MCI for retentostat cultures might further enhance production of cheese-like aroma compounds. Aroma formation in a milli-cheese model system was compared with retentostat cultures using the chemically defined medium and batch cultures using hydrolysed MCI (Chapter 6) revealing that fatty acids and their corresponding aroma compounds were underrepresented. Therefore, it could be interesting to add fatty acids in the chemically defined medium and hydrolysed MCI to enhance aroma formation during the retentostat cultivations. Fatty acids are known to induce the $\beta$ oxidation system of L. lactis ( $\mathrm{Li}$ and $\mathrm{Ma}, 2013$ ) conceivably further boosting aroma formation and in particular the production of methyl ketones and secondary alcohols in these retentostat cultivations.

Another interesting technique to enhance aroma formation in retentostat cultures is the use of multiple strains and species in retentostat co-cultures. In this study, we investigated the feasibility of this method with two species of dairy lactic acid bacteria: L. lactis and Lc. mesenteroides (Chapter 7). Both species were retained and stayed viable in the co-cultures and the ratio of $L$. lactis and $L c$. mesenteroides was stably maintained at 100:1 from approximately 1 week till the end of the cultivation. In Dutch-type cheeses made with these species, L. lactis is dominating at a similar ratio (Erkus et al., 2013, Frantzen et al., 2017). Despite representing the cheese microbial population, interactions between the species was limited, possibly due to this large difference in abundance. Different ratios could severely affect aroma formation and the interaction between species as demonstrated for co-cultures of Saccharomyces cerevisiae and Cyberlindera fabianii (van Rijswijck et al., 2017). In the future, the expected ratio of the species should be taken into account in the selection of strains and species for retentostat co-cultures, which can be predicted based on growth parameters obtained from retentostat monocultures using the model described in Chapter 7. In addition, this model allows to predict the effect of the inoculation ratio (ratio at start of retentostat cultivation) on the final ratio during retentostat cultivations. The model revealed that by greatly increasing the relative abundance of $L c$. mesenteroides FM06 compared to L. lactis FM03-V1 at the start of the retentostat (from $1: 1$ to $1,000: 1$ ), the relative abundance of LC. mesenteroides FM06 could be slightly increased from approximately $1: 35$ to $1: 10$ (Fig. 10.5A). Moreover, the model revealed that when microorganisms have equal growth characteristics, the final ratio in the retentostat is equal to the inoculation ratio (Fig. 10.5B). These two examples 

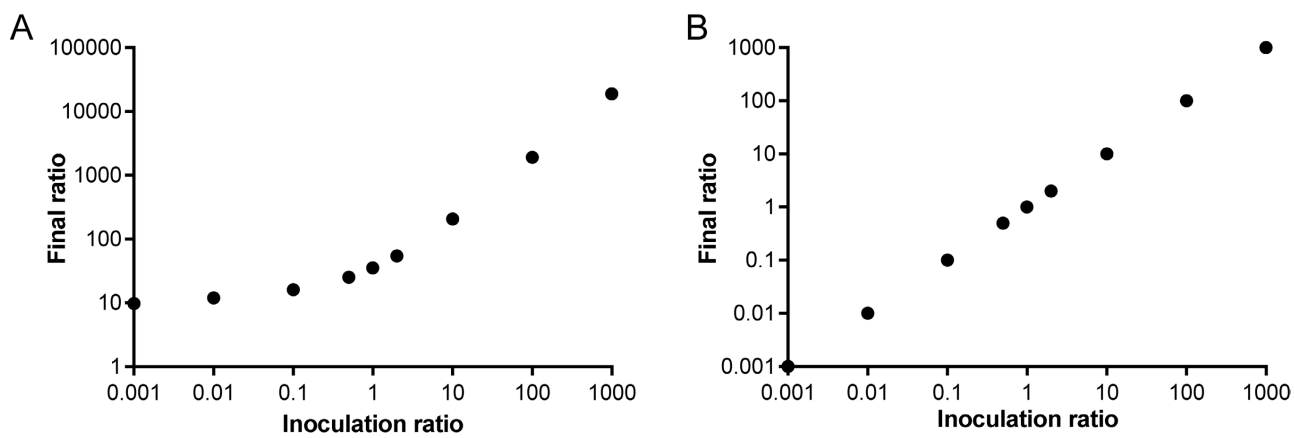

Figure 10.5: Predicted effect of the inoculation ratio (ratio at start) on the final ratio (ratio at end) during retentostat co-cultivation. A: Prediction for co-cultivation of L. lactis FM03$\mathrm{V} 1$ and $L c$. mesenteroides FM06. Ratio is defined as L. lactis / LC. mesenteroides B: Prediction for co-cultivation of two microorganisms with equal growth characteristics.

indicate that the inoculation ratio can be varied to fine-tune the final ratio of the microorganisms and thereby to fine-tune aroma formation in retentostat coculture.

Another interesting approach using retentostat co-cultures to enhance aroma formation is to combine lactic acid producing L. lactis spp. with propionibacteria, reflecting the community in Swiss-type cheese. Because the propionibacteria can consume the lactate and convert it into propionate generating energy, a more similar abundance of both bacterial species is expected.

Although retentostat cultivation is a unique cultivation method to study microorganisms at near-zero growth rates, some considerations should be taken into account including the coupling of growth rate to the time in the bioreactor and the biomass concentration. With the partial cell recycling chemostat (Chapter 9) growth rates can be controlled (also at near-zero growth rates) and are not coupled to the time in the reactor. Thereby, this system allows to study the effect of the growth rate and time in the bioreactor independently from each other to provide insight into questions such as when cells switch to a viable but nonculturable state: below a certain nutrient availability or after a certain time with nutrient limitation. By changing the feed rate, the biomass concentration can be controlled in partial cell recycling chemostats to study for instance quorum sensing, which could affect production of volatile compounds (Kesarwani et al., 2011). Furthermore, due to its time-independency, the partial cell recycling chemostat is more reproducible than retentostat cultivation because it is not affected by small disturbances by for instance sampling, clogging of the filter or changes in the feed rate. This makes it also more suitable for omics studies. The partial cell recycling chemostat also has advantages as production platform. Like 
the retentostat, dilution rates can be higher than the maximum growth rate and high biomass concentrations can be obtained. Moreover, due to its time independency, production will be stable in time and cells will remain viable because the growth rates can be controlled as values just above zero ( $>99 \%$, Chapter 9). Finally, to minimise the time to reach the steady state in the partial cell recycling chemostat, processes can start at a high feed rate to quickly produce biomass. When the biomass concentration approaches its desired value, the feed rate can be decreased to its corresponding value.

In conclusion, the work described in this thesis demonstrates the importance of slowly growing bacteria for aroma formation and provides new opportunities for enhanced production of aroma compounds. Retentostat cultivation of the lactic acid bacteria revealed novel physiological, genetic and metabolic adaptations that increased their survival under extreme nutrient limitation, which helps to understand how microbes adapt in food fermentation processes as well as in natural environments in which nutrient are often scarce. 


\section{References}

Arbige, M. and Chesbro, W. (1982). relA and related loci are growth rate determinants for Escherichia coli in a recycling fermenter. Microbiology 128(4): 693-703.

Audrain, B., Farag, M. A., Ryu, C.-M. and Ghigo, J.-M. (2015). Role of bacterial volatile compounds in bacterial biology. FEMS Microbiol. Rev. 39(2): 222-233.

Ayrapetyan, M., Williams, T. C. and Oliver, J. D. (2015). Bridging the gap between viable but non-culturable and antibiotic persistent bacteria. Trends Microbiol. 23(1): 713.

Boender, L. G. M., van Maris, A. J. A., de Hulster, E. A. F., Almering, M. J. H., van der Klei, I. J., Veenhuis, M., de Winde, J. H., Pronk, J. T. and Daran-Lapujade, P. (2011). Cellular responses of Saccharomyces cerevisiae at near-zero growth rates: transcriptome analysis of anaerobic retentostat cultures. FEMS Yeast Res. 11(8): 603-620.

Brock, T. D. (1971). Microbial growth rates in nature. Bacteriol. Rev. 35(1): 39-58.

Campbell, J. W., Morgan-Kiss, R. M. and E. Cronan, J. (2003). A new Escherichia coli metabolic competency: growth on fatty acids by a novel anaerobic $\beta$-oxidation pathway. Mol. Microbiol. 47(3): 793-805.

Cavanagh, D., Fitzgerald, G. F. and McAuliffe, O. (2015). From field to fermentation: the origins of Lactococcus lactis and its domestication to the dairy environment. Food Microbiol. 47: 45-61.

Chambellon, E. and Yvon, M. (2003). CodY-regulated aminotransferases AraT and BcaT play a major role in the growth of Lactococcus lactis in milk by regulating the intracellular pool of amino acids. Appl. Environ. Microbiol. 69(6): 3061-3068.

Chambellon, E., Rijnen, L., Lorquet, F., Gitton, C., van Hylckama Vlieg, J. E., Wouters, J. A. and Yvon, M. (2009). The D-2-hydroxyacid dehydrogenase incorrectly annotated PanE is the sole reduction system for branched-chain 2-keto acids in Lactococcus lactis. J. Bacteriol. 191(3): 873-881.

Crow, V. L., Coolbear, T., Gopal, P. K., Martley, F. G., McKay, L. L. and Riepe, H. (1995). The role of autolysis of lactic acid bacteria in the ripening of cheese. Int. Dairy $\mathrm{J}$. 5(8): 855-875.

den Hengst, C. D., van Hijum, S. A., Geurts, J. M., Nauta, A., Kok, J. and Kuipers, O. P. (2005). The Lactococcus lactis CodY regulon identification of a conserved cisregulatory element. J. Biol. Chem. 280(40): 34332-34342.

Desbois, A. P. and Smith, V. J. (2010). Antibacterial free fatty acids: activities, mechanisms of action and biotechnological potential. Appl. Microbiol. Biotechnol. 85(6): 16291642.

Ercan, O., Smid, E. J. and Kleerebezem, M. (2013). Quantitative physiology of Lactococcus lactis at extreme low-growth rates. Environ. Microbiol. 15(8): 2319-2332.

Ercan, O., Wels, M., Smid, E. J. and Kleerebezem, M. (2015). Molecular and metabolic adaptations of Lactococcus lactis at near-zero growth rates. Appl. Environ. Microbiol. 81(1): 320-331.

Erkus, O., de Jager, V. C. L., Spus, M., van Alen-Boerrigter, I. J., van Rijswijck, I. M. H., Hazelwood, L., Janssen, P. W. M., van Hijum, S. A. F. T., Kleerebezem, M. and Smid, E. J. (2013). Multifactorial diversity sustains microbial community stability. ISME J 7(11): 2126-2136.

Erkus, O., de Jager, V. C. L., Geene, R. T. C. M., van Alen-Boerrigter, I., Hazelwood, L., van Hijum, S. A. F. T., Kleerebezem, M. and Smid, E. J. (2016). Use of propidium monoazide for selective profiling of viable microbial cells during Gouda cheese ripening. Int. J. Food Microbiol. 228(Supplement C): 1-9.

Fox, P. F., Guinee, T. P., Cogan, T. M. and McSweeney, P. L. H. (2017). Biochemistry of cheese ripening. In Fox, P. F., Guinee, T. P., Cogan, T. M. and McSweeney, P. L. H. (Eds.), Fundamentals of cheese science (pp. 391-442). Boston, MA: Springer.

Franken, L. E., Oostergetel, G. T., Pijning, T., Puri, P., Arkhipova, V., Boekema, E. J., Poolman, B. and Guskov, A. (2017). A general mechanism of ribosome dimerization revealed by single-particle cryo-electron microscopy. Nature communications $8(1)$ : 722. 
Frantzen, C. A., Kot, W., Pedersen, T. B., Ardö, Y. M., Broadbent, J. R., Neve, H., Hansen, L. H., Dal Bello, F., Østlie, H. M., Kleppen, H. P., Vogensen, F. K. and Holo, H. (2017). Genomic characterization of dairy associated Leuconostoc species and diversity of Leuconostocs in undefined mixed mesophilic starter cultures. Front. Microbiol. 8: 132.

Ganesan, B., Dobrowolski, P. and Weimer, B. C. (2006). Identification of the leucine-to-2methylbutyric acid catabolic pathway of Lactococcus lactis. Appl. Environ. Microbiol. 72(6): 4264-4273.

Gardner, A., West, S. A. and Griffin, A. S. (2007). Is bacterial persistence a social trait? PLoS One 2(8): e752.

Goel, A., Eckhardt, T. H., Puri, P., de Jong, A., Branco dos Santos, F., Giera, M., Fusetti, F., de Vos, W. M., Kok, J., Poolman, B., Molenaar, D., Kuipers, O. P. and Teusink, B. (2015). Protein costs do not explain evolution of metabolic strategies and regulation of ribosomal content: does protein investment explain an anaerobic bacterial Crabtree effect? Mol. Microbiol. 97(1): 77-92.

Goffin, P., van de Bunt, B., Giovane, M., Leveau, J. H. J., Höppener-Ogawa, S., Teusink, B. and Hugenholtz, J. (2010). Understanding the physiology of Lactobacillus plantarum at zero growth. Mol. Syst. Biol. 6(413).

Guédon, E., Serror, P., Ehrlich, S. D., Renault, P. and Delorme, C. (2001). Pleiotropic transcriptional repressor CodY senses the intracellular pool of branched-chain amino acids in Lactococcus lactis. Mol. Microbiol. 40(5): 1227-1239.

Hoehler, T. M. and Jorgensen, B. B. (2013). Microbial life under extreme energy limitation. Nat. Rev. Microbiol. 11(2): 83-94.

Kai, M., Haustein, M., Molina, F., Petri, A., Scholz, B. and Piechulla, B. (2009). Bacterial volatiles and their action potential. Appl. Microbiol. Biotechnol. 81(6): 1001-1012.

Kempes, C. P., van Bodegom, P. M., Wolpert, D., Libby, E., Amend, J. and Hoehler, T. (2017). Drivers of bacterial maintenance and minimal energy requirements. Front. Microbiol. 8(31).

Kesarwani, M., Hazan, R., He, J., Que, Y., Apidianakis, Y., Lesic, B., Xiao, G., Dekimpe, V., Milot, S., Deziel, E., Lépine, F. and Rahme, L. G. (2011). A quorum sensing regulated small volatile molecule reduces acute virulence and promotes chronic infection phenotypes. PLoS Pathog. 7(8): e1002192.

Kinderlerer, J. L. (1993). Fungal strategies for detoxification of medium chain fatty acids. Int. Biodeterior. Biodegradation 32(1): 213-224.

Koch, A. L. (1971). The adaptive responses of Escherichia coli to a feast and famine existence. Adv. Microb. Physiol. 6: 147-217.

Lahtvee, P.-J., Seiman, A., Arike, L., Adamberg, K. and Vilu, R. (2014). Protein turnover forms one of the highest maintenance costs in Lactococcus lactis. Microbiology 160(7): 1501-1512.

Lawrence, R. and Hawke, J. (1968). The oxidation of fatty acids by mycelium of Penicillium roqueforti. Microbiology 51(2): 289-302.

Lennon, J. T. and Jones, S. E. (2011). Microbial seed banks: the ecological and evolutionary implications of dormancy. Nature Reviews Microbiology 9(2): 119.

Lewis, H. L. and Darnall, D. W. (1970). Fatty acid toxicity and methyl ketone production in Aspergillus niger. J. Bacteriol. 101(1): 65-71.

$\mathrm{Li}$, L. and Ma, Y. (2013). Effect of fatty acids on the $\beta$-oxidation system and thioesterase of Lactococcus lactis subspecies lactis. J. Dairy Sci. 96(4): 2003-2010.

Maisonneuve, E., Castro-Camargo, M. and Gerdes, K. (2013). (p)ppGpp controls bacterial persistence by stochastic induction of toxin-antitoxin activity. Cell 154(5): 11401150.

McSweeney, P. L. H. (2004). Biochemistry of cheese ripening. Int. J. Dairy Technol. 57(23): $127-144$

McSweeney, P. L. H. (2007). Cheese manufacture and ripening and their influence on cheese flavour In Weimer, B. C. (Ed.), Improving the Flavour of Cheese (pp. 1-25). Cambridge, England: Woodhead Publishing Limited.

Mogk, A., Bukau, B. and Kampinga, H. H. (2018). Cellular handling of protein aggregates by disaggregation machines. Mol. Cell 69(2): 214-226. 
Molenaar, D., van Berlo, R., de Ridder, D. and Teusink, B. (2009). Shifts in growth strategies reflect tradeoffs in cellular economics. Mol. Syst. Biol. 5(1).

Morita, R. Y. (1993). Bioavailability of energy and the starvation state. In Kjelleberg, S. (Ed.), Starvation in Bacteria (pp. 1-23). Boston, MA: Springer US.

Morita, R. Y. (1997). Bacteria in oligotrophic environments: starvation-survival lifestyle. New York: Chapman \& Hall.

Oku, H. and Kaneda, T. (1988). Biosynthesis of branched-chain fatty acids in Bacillus subtilis. A decarboxylase is essential for branched-chain fatty acid synthetase. $J$. Biol. Chem. 263(34): 18386-18396.

Overkamp, W., Ercan, O., Herber, M., van Maris, A. J. A., Kleerebezem, M. and Kuipers, O. P. (2015). Physiological and cell morphology adaptation of Bacillus subtilis at nearzero specific growth rates: a transcriptome analysis. Environ. Microbiol. 17(2): 346363.

Panikov, N. S., Mandalakis, M., Dai, S., Mulcahy, L. R., Fowle, W., Garrett, W. S. and Karger, B. L. (2015). Near-zero growth kinetics of Pseudomonas putida deduced from proteomic analysis. Environ. Microbiol. 17(1): 215-228.

Parsell, D. A., Kowal, A. S., Singer, M. A. and Lindquist, S. (1994). Protein disaggregation mediated by heat-shock protein Hspl04. Nature 372(6505): 475.

Puri, P., Eckhardt, T. H., Franken, L. E., Fusetti, F., Stuart, M. C. A., Boekema, E. J., Kuipers, O. P., Kok, J. and Poolman, B. (2014). Lactococcus lactis YfiA is necessary and sufficient for ribosome dimerization. Mol. Microbiol. 91(2): 394-407.

Ruggirello, M., Cocolin, L. and Dolci, P. (2016). Fate of Lactococcus lactis starter cultures during late ripening in cheese models. Food Microbiol. 59: 112-118.

Schirmer, E. C., Glover, J. R., Singer, M. A. and Lindquist, S. (1996). HSP100/Clp proteins: a common mechanism explains diverse functions. Trends Biochem. Sci. 21(8): 289296.

Schmidt, R., Cordovez, V., De Boer, W., Raaijmakers, J. and Garbeva, P. (2015). Volatile affairs in microbial interactions. The ISME journal 9(11): 2329.

Smid, E. J. and Kleerebezem, M. (2014). Production of aroma compounds in lactic fermentations. Annu. Rev. Food Sci. Technol. 5(1): 313-326.

Smit, G., Smit, B. A. and Engels, W. J. M. (2005). Flavour formation by lactic acid bacteria and biochemical flavour profiling of cheese products. FEMS Microbiol. Rev. 29(3): 591-610.

Stuart, M. R., Chou, L. S. and Weimer, B. C. (1999). Influence of carbohydrate starvation and arginine on culturability and amino acid utilization of Lactococcus lactis subsp. lactis. Appl. Environ. Microbiol. 65(2): 665-673.

Sugimoto, S., Abdullah Al, M. and Sonomoto, K. (2008). Molecular chaperones in lactic acid bacteria: physiological consequences and biochemical properties. Journal of Bioscience and Bioengineering 106(4): 324-336.

Thomas, T. D., Ellwood, D. C. and Longyear, V. M. C. (1979). Change from homo-to heterolactic fermentation by Streptococcus lactis resulting from glucose limitation in anaerobic chemostat cultures. J. Bacteriol. 138(1): 109-117.

van Rijswijck, I. M. H., Wolkers - Rooijackers, J. C. M., Abee, T. and Smid, E. J. (2017). Performance of non-conventional yeasts in co-culture with brewers' yeast for steering ethanol and aroma production. Microb. Biotechnol. 10(6): 1591-1602.

Yu, A.-Q., Pratomo Juwono, N. K., Leong, S. S. J. and Chang, M. W. (2014). Production of fatty acid-derived valuable chemicals in synthetic microbes. Frontiers in Bioengineering and Biotechnology 2(78).

Zolkiewski, M. (1999). ClpB cooperates with DnaK, DnaJ, and GrpE in suppressing protein aggregation a novel multi-chaperone system from Escherichia coli. J. Biol. Chem. 274(40): 28083-28086. 



\section{Summary}

Acknowledgements

About the author

\section{List of publications}

Overview of training activities 



\section{Summary}

Lactic acid bacteria ( $L A B$ ) are extensively used for the production of fermented foods from both animal and plant origin, such as cheese, yoghurt, kimchi and sauerkraut. Their predominant function in these processes is to produce organic acids, mainly lactic acid, thereby lowering the $\mathrm{pH}$, which contributes to the safety and shelf life of the product. Nowadays, many fermented foods are made using starter cultures for a consistently safe product of constant quality. In Dutch-type cheeses, the starter cultures consist of various strains of mainly two species: Lactococcus lactis and Leuconostoc mesenteroides. L. lactis subsp. cremoris and L. lactis subsp. lactis are considered to be the main acid producers that dominate during the early stages of cheese manufacturing when nutrients are abundant. During cheese ripening, nutrients are scarce and LAB encounter long periods of nutrient limitation leading to slow growth. In particular, L. lactis biovar diacetylactis and Lc. mesenteroides survive these periods of extreme nutrient limitation and still contribute to aroma formation. In this thesis, the dairy-isolated strains Lactococcus lactis biovar diacetylactis FM03P and Leuconostoc mesenteroides FM06 were studied focussing on the effect of near-zero growth rates on their quantitative physiology and aroma formation capacity. Moreover, possible strategies to enhance aroma formation at near-zero growth rates are described.

The genomes of both lactic acid bacteria strains were sequenced, including the plasmids they harboured, (Chapter 2 ) revealing that their genomes carried clear signatures of adaptation to the dairy environment.

The plasmids of L. lactis FM03P and the genes they carried were further characterised in Chapter 3. Plasmid-encoded functions that were identified include lactose utilisation, citrate uptake, oligopeptide uptake and peptide degradation, bacteriophage resistance, uptake of cations (magnesium and manganese), exopolysaccharide production, and stress resistance. Acquisition of the plasmids most likely facilitated the adaption of these strains to the dairy environment. Interestingly, some plasmids were already lost in a single propagation step, signifying their instability in the absence of a selection pressure and demonstrating that propagation should be minimised when studying dairy isolates of L. lactis.

In L. lactis, citrate utilisation has been linked to the production of the important aroma compounds diacetyl and acetoin. In L. lactis biovar diacetylactis, the limiting step in citrate utilisation is the transport of citrate across the cell membrane, which is facilitated by the plasmid-encoded citrate permease (CitP). 
Although it is known citP expression is induced at low $\mathrm{pH}$, the effect of citrate is still under debate. Moreover, the role of the plasmid copy number (number of plasmid copies per chromosome) has never been taken into account. In Chapter 4, we systematically analysed regulation of citrate utilisation by $\mathrm{pH}$, nutrient limitation and presence of citrate at four different levels: i) plasmid copy number, ii) citP transcription, iii) citP mRNA processing and iv) citrate utilisation capacity. Induction of the citP gene at low $\mathrm{pH}$ was confirmed, but citP expression increased even more in the presence of citrate. In cells grown at low $\mathrm{pH}$ or under amino acid limitation, also the copy number of the citP-containing plasmid slightly increased. No significant effects on citP mRNA processing were found. Due to the increased citP expression, the citrate utilisation rate increased from approximately 1 to $65 \mu \mathrm{mol} . \mathrm{min}^{-1} . \mathrm{gDW}^{-1}$ and also the production of acetoin increased significantly. This knowledge was used in other conditions to select conditions that improved citrate-driven flavour formation.

In cheese manufacturing, aroma compounds are mainly formed by the bacteria in the ripening phase in which nutrients are limited resulting in severely reduced growth rates. To investigate if reduced nutrient availabilities and low growth rates are required for the formation of aroma compounds, L. lactis FM03-V1 was grown for 2 weeks in batch and retentostat cultures and in a milli-cheese model system. Subsequently, aroma compounds were analysed by headspace solid phase microextraction gas chromatography mass spectrometry (Chapter 5). While batch cultures were performed with milk, hydrolysed micellar casein isolate (MCI) and a chemically defined medium (CDM), retentostat cultures were only performed with CDM. Despite the use of CDM, aroma production in retentostat cultivations had the biggest qualitative overlap with aroma production in the milli-cheese model system demonstrating that low growth rates are required to produce cheeserelated compounds. In total, 52 known cheese compounds were produced in retentostat cultures. In cultures with CDM and $\mathrm{MCI}$, free fatty acids and their corresponding degradation products were underrepresented compared to the milli-cheeses. Therefore, addition of free fatty acids to MCI and CDM might help to enhance flavour formation, thereby better resembling flavour formation in cheese.

To further study the effect of near-zero growth rates on the aroma formation capacity and quantitative physiology, L. lactis FM03-V1 was grown for 5 weeks in retentostat cultures (Chapter 6). Growth rates decreased to less than $0.001 \mathrm{~h}^{-1}$, while the viability remained above $80 \%$. Interestingly, a large fraction of the cells lost the ability to grow in M17 plates, indicating that they entered a viable but non-culturable state. Dynamic modelling revealed that the maintenance coefficient of this dairy strain decreased 7-fold at near-zero growth rates 
compared to high growth rates. Most likely, the bacteria saved energy by limiting energy used for protein turnover. Changes in aroma formation in the retentostat culture resembled biochemical changes occurring during cheese ripening, such as amino acid and fatty acid catabolism. Analysis of complete and cell-free samples revealed that lipophilic compounds accumulated in the cells, most likely in the cell membranes.

Starter cultures for cheese production often consist of multiple strains and species, which could result in a higher aroma complexity. To investigate if retentostat cocultures could further enhance aroma formation, L. lactis FM03-V1 and LC. mesenteroides FM06 were grown in retentostat mono- and co-cultures (Chapter 7). Both species had similar physiological responses to near-zero growth rates, including that a large fraction was viable but not culturable and that the maintenance coefficient decreased approximately 7-fold. However, L. lactis reached a higher biomass concentration in retentostat mono-cultures due to a higher ATP yield on substrate, a higher biomass yield on ATP and a lower maintenance requirement. A dynamic model was developed to predict biomass accumulation in retentostat co-cultures. This model predicted the biomass accumulation very well with $L$. lactis dominating in the retentostat co-cultures (ratio 100:1). Aroma compounds specific for both species were identified in retentostat mono-cultures. No additional unique aroma compounds were identified in the co-cultures, although 2 out of 5 compounds specific for $L C$. mesenteroides were found in the co-cultures despite its low abundance. This indicates that a similar or even higher aroma complexity could be obtained by mixing two retentostat mono-cultures.

Lactic acid bacteria can carry multiple plasmids affecting their performance in dairy fermentations. The expression of plasmid-borne genes and the activity of the corresponding proteins is severely affected by changes in the number of plasmid copies. Therefore, we analysed plasmid copy numbers in a wide variety of dairy-related conditions, including near-zero growth rates, typical for cheese ripening (Chapter 8 ). The copy numbers of theta-type replicating plasmids were negatively correlated with the size of the plasmids. Plasmid copy numbers were remarkably stable in the extremely wide range in growth rates $\left(0.0003 \mathrm{~h}^{-1}\right.$ to $\left.0.6 \mathrm{~h}^{-1}\right)$, suggesting strict control of the plasmid copy number even at extreme nutrient restriction. Copy numbers were also hardly affected by varying the $\mathrm{pH}$ value, the nutrient limitation or the presence of citrate, signifying the stability in copy number of the plasmids.

Although retentostat cultivation is a unique cultivation method to study microorganisms at near-zero growth rates, it has some disadvantages as 
production platform in particular due to the time dependency. Partial cell recycling chemostats overcome this problem, while near-zero growth rates can still be obtained as demonstrated in Chapter 9. Using a partial cell recycling chemostat cultivation, the decreased maintenance requirement of $L$. lactis at near-zero growth rates was confirmed. Moreover, it was demonstrated that particular aroma compounds were affected by the growth rate with the studied range $(0.0025$ to $0.025 \mathrm{~h}^{-1}$. Finally, the potential of partial cell recycling chemostat cultivation as production platform and as unique research tool are discussed.

The results of the experimental chapters (Chapter 2-9) were further discussed in Chapter 10, including a comparison with transcriptomics data of retentostat cultures of the plant-associated L. lactis KF147. This showed that the decreased maintenance requirement at near-zero growth rates might be explained by switching from degradation and re-synthesis of misfolded proteins to disaggregation and refolding of misfolded proteins with the ClpB-DnaK system. The function of aroma formation in lactic acid bacteria was also discussed, in particular in relation to their possible role as release valve for the excess of branched-chain amino acids.

In conclusion, this thesis described and revealed new physiological, genetic and metabolic adaptations of lactic acid bacteria to near-zero growth rates. This helps to understand how microbes adapt in food fermentation processes as well as in natural environments in which nutrients are often scarce. Moreover, this work demonstrates the importance of slow growth for the production of aroma compounds by lactic acid bacteria and provides new opportunities for enhanced production of aroma compounds. 


\section{Acknowledgements}

This thesis describes the physiological changes in lactic acid bacteria when they encounter environments with extreme nutrient stress. Also during my $\mathrm{PhD}$ research, I sometimes encountered stressful conditions and I am grateful for all the people that supported, inspired and motivated me during these last four years. This thesis would not be possible without your help!

Allereerst wil ik mijn promotoren Eddy en Tjakko heel erg bedanken. Eddy, bedankt voor je vertrouwen in mij, zowel aan het begin door mij deze prachtige kans te geven als gedurende de afgelopen 4 jaar. Bedankt voor het delen van je kennis en expertise. De deur stond altijd open voor al mijn vragen en jouw enthousiasme voor de wereld van de microbiologie is een grote inspiratiebron voor mij geweest. Tjakko, jouw explosie aan ideeën tijdens meetings en de kritische blik op de manuscripten waren van onschatbare waarde. Jullie waren het beste duo dat ik me kan voorstellen en vulden elkaar perfect aan, waardoor ik ben gegroeid op zowel persoonlijk als professionele vlak. Bedankt!

I want to thank Arla Foods (Denmark) for financially supporting this PhD research and for the fruitful and inspiring progress meetings. Søren, thank you for sharing your tremendous knowledge about cheese and for your optimistic view that always gave a new boost. Sirina and Ellen, thank you for your roles as project leaders and your input in the discussions. Mette, thank you for isolation the strains and for developing the MCI medium.

In meerdere hoofdstukken speelt de GC-MS een grote rol. Irma en Judith, zonder jullie hulp en kennis was het niet zo eenvoudig om de GC-MS te gebruiken en de data te analyseren. Bedankt voor alle tijd en moeite die jullie erin gestoken om dit platform bij FHM op te zetten!

Ik wil graag mijn grote dank betuigen aan Prof. Dr Richard van Kranenburg, Prof. Dr Jeroen Hugenholtz, Prof. Dr Pascale Daran-Lapujade en Dr Mariela Serrano voor jullie tijd om mijn proefschrift kritisch te beoordelen. Bedankt voor het accepteren van de uitnodiging om deel te zijn van de thesis committee.

I want to express my sincere gratitude to all my current and former colleagues at the Laboratory of Food Microbiology. You make this place to a nice work environment. Marcel, bedankt voor het faciliteren van de zomerbarbeques en de labuitjes. Ze vormen een belangrijke bedrage aan de sfeer in de groep. Gerda, bedankt voor je hulp bij de administratieve werkzaamheden. Ingrid, bedankt voor het op orde houden van de keuken, het bijhouden van de voorraden en het regelen van de bestellingen. Judith, met jouw optimisme en energie sprong je altijd overal bij waar nodig. Bedankt! Maciej and Irma, thanks for taking care of the 
fermentation lab as lab heads and for making this place to a wonderful place to do my experiments.

Also thanks to all my follow PhD students, Jeroen, Irma, Natalia, Yue, Frank, Evelien, Maciej, Bernard, Diego, Angela, Anneloes, Maren, Jasper, Pjotr, Andy, James, Karin, Hasmik, Alicja, Monica, Diah and Joanna. Thanks for the unforgettable PhD trips, lunch breaks, drinks, dinners, WE days, PhD weekend, advices and fun at the department. Jeroen, ik heb een aantal jaar met veel plezier het kantoor met jou gedeeld. Bedankt voor al je hulp en adviezen en het delen van je kennis. Ik heb genoten van de vele discussies op het gebied van selectie en evolutie.

Big thanks to all the MSc and BSc students that worked on this project: Yorick, Artemis, Elisa, Pallavi, Marcel, Casper, Emma, Sylviani, Diego and Reinier. I am glad that I had the opportunity to supervise you all. I learned a lot from your different personalities and your extra hands in the lab were indispensable to develop all the methods to obtain all the results described in this thesis. Thanks for being part of this team and I wish you all the best in the future.

In het bijzonder wil ik mijn twee paranimfen bedanken. Jeroen, het is vanzelfsprekend dat jij aan mijn zijde staat vandaag. We deelden een kantoor, onze gesprekken en discussies zijn van onkenbare waarde geweest voor mij persoonlijk als voor dit proefschrift en je stond altijd klaar om te helpen en mijn vragen te beantwoorden. Ik heb veel van je geleerd, bedankt! Yue, it is also makes sense that you stand with me today. We met already during our internship at Dyadic before I started my PhD and I am thankful that we met again. We both worked with lactic acid bacteria and I am happy that we could help each other. You boosted my confidence with your kind words and thankfulness and you showed me the importance of having colleagues to share knowledge. I want to thank both of you for all the fun we had and good luck with finishing your own PhD. I am confident that you can do it!

Natuurlijk kan ik dit dankwoord niet afsluiten zonder mijn (schoon)familie en vrienden te bedanken. Ik voel me een geluksvogel om jullie om me heen te hebben. Ondanks dat dit proefschrift inhoudelijk ver weg staat van de meeste van jullie, verdienen jullie steun, liefde en vertrouwen hier zeker een plekje. Vrienden van mijn studie, elk zijn we onze eigen weg gegaan maar ik geniet nog steeds van de momenten dat we elkaar weer zien. Bedankt!

Pap en mam, bedankt voor jullie onvoorwaardelijke liefde, goede zorgen en vertrouwen in mij. Ik ben dankbaar dat jullie mij altijd hebben gesteund in mijn 
keuzes. Mam, onbewust heb je waarschijnlijk mijn interesse voor microbiologie aangewakkerd, wat mij uiteindelijk op deze plek heeft gebracht.

Liefste Sophie, ik ben zo blij dat we samen mogen zijn. Jouw liefde, vertrouwen, steun en goede zorgen maken het leven tot een feest. De momenten van ontspanning samen zorgen ervoor dat ik er weer tegen kan en elke dag dat ik naar huis ga kijk ik uit je weer te zien. Door elkaars aanwezigheid mogen we onszelf steeds beter leren kennen en groeien. Het is zo fijn om samen met jou te zijn en ik hoop nog vele avonturen met je te beleven. 



\section{About the author}

Oscar van Mastrigt was born on 10 September 1990 in Barendrecht, the Netherlands. In 2008, he graduated from secondary school at Calvijn in Rotterdam. In the same year, he started the BSc Life Science \& Technology at the Technical University Delft and Leiden University finishing with a BSc thesis at Industrial Microbiology at the TU Delft. After completion cum laude in 2011, he started the MSc Life Science \& Technology at the TU Delft with specialisations Cell Factory and Biochemical Engineering. His MSc thesis was conducted at Cell Systems Engineering (formerly known as Bioprocess Engineering) at the TU Delft focussing on transport and metabolism of fumaric acid transport in Saccharomyces cerevisiae under aerobic condition at low pH. He conducted his internship at Dyadic Netherlands (currently DuPont Industrial Biosciences) in Wageningen focussing on characterisation and comparison of production strains of Myceliophthora thermophila $\mathrm{C} 1$ with respect to growth and protein production in order to optimise production processes with $\mathrm{C} 1$. After he graduated cum laude in 2013, he got the opportunity to start his PhD project, entitled: "Enhancing aroma production by lactic acid bacteria at near-zero growth rates: a retentostat approach", at the Laboratory of Food Microbiology at Wageningen University \& Research. The results of this work is described in this thesis. Currently, Oscar is working as Postdoc at the Laboratory of Food Microbiology at Wageningen University \& Research. 



\section{List of publications}

van Mastrigt, O., Abee, T., Smid, E. J. (2017). Complete genome sequences of Lactococcus lactis subsp. lactis bv. diacetylactis FM03 and Leuconostoc mesenteroides FM06 isolated from cheese, Genome Announc. 5: e00633-17.

van Mastrigt, O., Mager, E. E., Jamin, C., Abee, T., Smid, E. J. (2018a). Citrate, low $\mathrm{pH}$ and amino acid limitation induce citrate utilization in Lactococcus lactis biovar diacetylactis, Microb. Biotechnol. 11:369-380.

van Mastrigt, O., Abee, T., Lillevang, S. K., Smid, E. J. (2018b). Quantitative physiology and aroma formation of a dairy Lactococcus lactis at near-zero growth rates, Food Microbiol. 73:216-226.

van Mastrigt, O., Lommers, M. M. A. N., de Vries, Y. C., Abee, T., Smid, E. J. (2018c). Dynamics in copy numbers of five plasmids of a dairy Lactococcus lactis under dairy-related conditions including near-zero growth rates, Appl. Environ. Microbiol. 84:e00314-18.

van Mastrigt, O., Gallegos Tejeda, D., Kristensen, M. N., Abee, T., Smid, E. J. (2018d). Aroma formation during cheese ripening is best resembled by Lactococcus lactis retentostat cultures, Microb. Cell Fact. 17:104

van Mastrigt, O., Di Stefano, E., Hartono, S., Abee, T., Smid, E. J. Large plasmidome of dairy Lactococcus lactis subsp. lactis biovar diacetylactis FM03P encodes technological functions and appears highly unstable, submitted for publication.

van Mastrigt, O., Egas, R. A., Abee, T., Smid, E.J. Aroma formation in retentostat co-cultures of Lactococcus lactis biovar diacetylactis and Leuconostoc mesenteroides, submitted for publication.

van Rijswijck, I. M. H., van Mastrigt, O., Pijffers, G., Wolkers-Rooijackers, J. C. M., Abee, T., Zwietering, M. H., Smid, E.J. Dynamic modelling of brewers' yeast and Cyberlindnera fabianii co-culture behaviour for steering fermentation performance, submitted for publication. 



\section{Overview of completed training activities}

\section{Discipline specific activities}

\section{Courses}

Pilot plant trials

Arla Foods, Denmark 2014

Metabolic engineering

VLAG, Wageningen 2015

Food fermentation

VLAG, Wageningen 2016

Genetics and physiology of food-associated microorganisms

VLAG, Wageningen 2016

\section{Meetings and conferences}

LAB11, poster presentation and pitch presentation

Egmond aan Zee 2014

KNVM fall meeting Microbial Biotechnology

2015

IDF Parallel Symposia, Cheese Science \& Technology,

Delft

2016

oral presentation

$7^{\text {th }}$ FEMS congress, poster presentation

Dublin, Ireland

2017

LAB12, poster presentation

Valencia, Spain

2017

Egmond aan Zee

2017

KNVM fall meeting Microbial Biotechnology,

Delft

2017

poster presentation

\section{General courses}

VLAG PhD week

VLAG, Wageningen 2014

Basic statistics

Biometris, Wageningen 2015

Scientific writing

WGS, Wageningen 2016

Chemometrics

VLAG, Wageningen 2017

\section{Optional courses}

Preparation of research proposal

WUR, Wageningen 2014

$\mathrm{PhD}$ trip Ireland

FHM, Wageningen 2014

Organization PhD trip Italy

FHM, Wageningen 2017

PhD trip Italy

FHM, Wageningen 2017

Microbial population biology meetings

WUR, Wageningen 2017

Food Microbiology department seminars

FHM, Wageningen 2014- 
The research described in this thesis was financially supported by Arla Foods (Aarhus, Denmark)

Financial support from Wageningen University for printing the thesis is gratefully acknowledged.

Cover design by: Oscar van Mastrigt \& Sophie van Mastrigt - van Rijssel Printed by: Digiforce || ProefschriftMaken 
7

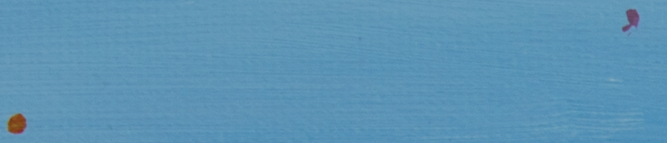

-

-

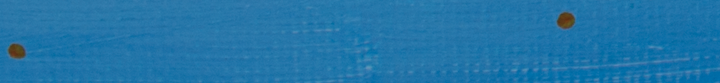

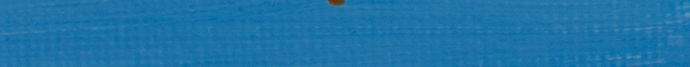

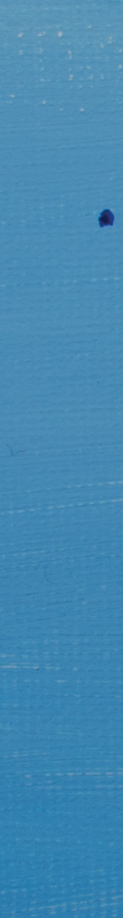

